\title{
MOLECULAR DETERMINATION OF MARINE IRON LIGANDS BY MASS SPECTROMETRY
}

\author{
By \\ Rene M. Boiteau \\ B.A., Northwestern University (2009) \\ MPhil., University of Cambridge (2010) \\ Submitted in partial fulfillment of the requirements for the degree of \\ Doctor of Philosophy \\ at the \\ MASSACHUSETTS INSTITUTE OF TECHNOLOGY \\ and the \\ WOODS HOLE OCEANOGRAPHIC INSTITUTION \\ February 2016 \\ (C) 2016 Rene Boiteau \\ All rights reserved. \\ The author hereby grants to MIT and WHOI permission to reproduce and \\ to distribute publicly paper and electronic copies of this thesis document \\ in whole or in part in any medium now known or hereafter created.
}

Signature of Author:

\begin{tabular}{lr}
\hline Certified by: $\begin{array}{r}\text { Joint Program in Oceanography } \\
\text { Massachusetts Institute of Technology } \\
\text { and Woods Hole Oceanographic Institution } \\
\text { (January 19, 2016) }\end{array}$ \\
\hline Accepted by: \\
$\begin{array}{r}\text { Daniel J. Repeta } \\
\text { Thesis Supervisor }\end{array}$ \\
\hline Chair, Joint Committee for Chemical Oceanography \\
Woods Hole Oceanographic Institution
\end{tabular}




\begin{abstract}
:
Marine microbes produce a wide variety of metal binding organic ligands that regulate the solubility and availability of biologically important metals such as iron, copper, cobalt, and zinc. In marine environments where the availability of iron limits microbial growth and carbon fixation rates, the ability to access organically bound iron confers a competitive advantage. Thus, the compounds that microbes produced to acquire iron play an important role in biogeochemical carbon and metal cycling. However, the source, abundance, and identity of these compounds are poorly understood. To investigate these processes, sensitive methodologies were developed to gain a compound-specific window into marine iron speciation by combining trace metal clean sample collection and chromatography with inductively coupled plasma mass spectrometry (LCICPMS) and electrospray ionization mass spectrometry (LC-ESIMS). Coupled with isotope pattern assisted search algorithms, these tools provide a means to quantify and isolate specific iron binding ligands from seawater and marine cultures, identify them based on their mass and fragmentation spectra, and investigate their metal binding kinetics.
\end{abstract}

Using these techniques, we investigated the distribution and diversity of marine iron binding ligands. In cultures, LC-ICPMS-ESIMS was used to identify new members of siderophore classes produced by marine cyanobacteria and heterotrophic bacteria, including synechobactins and marinobactins. Applications to natural seawater samples from the Pacific Ocean revealed a wide diversity of both known and novel metal compounds that are linked to specific nutrient regimes. Ferrioxamines B, E, and G were identified in productive coastal waters near California and Peru, in oligotrophic waters of the North and South Pacific Gyre, and in association with zooplankton grazers. Siderophore concentrations were up to five-fold higher in iron-deficient offshore waters $(9 \mathrm{pM})$ and were dominated by amphibactins, amphiphilic siderophores that partition into cell membranes. Furthermore, synechobactins were detected within nepheloid layers along the continental shelf. These siderophores reflect adaptations that impact dissolved iron bioavailability and thus have important consequences for marine ecosystem community structures and primary productivity. The ability to map and characterize these compounds has opened new opportunities to better understand mechanisms that link metals with the microbes that use them. 


\section{Acknowledgements:}

I am extremely grateful for the help and support of many amazing people throughout my time at MIT and WHOI. I would first like to thank my scientific mentors over the past five years, especially my advisor, Dan Repeta. Throughout graduate school, I was continuously inspired by Dan's hard work and creative problem solving in the lab, as well as his resilience, optimism, and good nature - It was a real pleasure to be a part of the Repeta lab. I thank him for always being ready and available to discuss science, read drafts, write letters, answer questions, and support me throughout my graduate career. I would also like to thank my committee members Mak Saito and Ed Boyle. One of my favorite aspects of the joint program was the entrenched attitude that any question can be answered if you can create the right tools for the job, and both Ed and Mak embody this idea - I consider myself extremely fortunate to have had their support and encouragement throughout my $\mathrm{PhD}$.

I was extremely fortunate to collaborate with many brilliant and talented researchers during my time in the Joint Program. Many thanks go to Jessica Fitzsimmons, for helping me get this project started with early mornings and late nights in the Isoprobe room. I'd like to thank Matt McIlvin and Gretchen Swarr for sharing a wealth of knowledge about mass spectrometry and chromatography and for always lending a helping hand when troubleshooting or trying out new methods. I would also like to thank the other members of the Repeta group, Jamie Becker, Chris Follett, Randie Bundy, and Prosper Zigah for making it a pleasure to work in the lab every day. Thanks to Craig McLean, Serena Dao, and Paige Kozlowski - it was a blast mentoring such talented and hard working undergraduates. Thanks to the faculty at WHOI who were always willing to share ideas and keep an open door, especially Bernhard Peucker-Ehrenbrink, Liz Kujawinski, Ben Van Mooy, and Tracy Mincer. Thanks to Dawn Moran, Krista Longnecker, and Melissa Soule for help with culturing, data processing, and high res mass spectrometry. I would also like to thank John Waterbury, Athena Archer, Eric Webb and Chris Suffridge for providing me with marine bacteria cultures that enabled me to develop the methods in this thesis. Thanks to the chief scientists who invited me to sail with them and collect samples for my work, especially Ken Bruland, James Moffett, Chris German, Sam Wilson, Tara Clemente, Carl Lamborg, and Mak Saito, and to many others that I had the opportunity to sail with, especially Gonzalo Carrasco, Chris Hayes, Ben Twining, Steve Baines, Claire Parker, Tyler Coale, Kristen Buck, and Geo Smith. One of the best features and greatest challenges of the Joint Program is the opportunity to take advantage of two amazing research institutions, and I am tremendously grateful to all of the labs at MIT that gave me a home in Cambridge. I am thankful for the help of Rick Kayser, Roger Summons, and Florence Shubotz in E25. Thanks to Penny Chisholm, Allison Coe, Paul Berube, Steve Biller, and Jessica Thompson for teaching me about the wonders of Prochlorococcus. Thanks to Martin Polz, Michael Cutler, Phil Arevalo, JanHendrick Hehemann, and Otto Cordero for helping me investigate siderophore production in Vibrio. Thanks to Ed Delong and Daniel Mende for helping me delve into the world of metagenomics. A special thanks goes to Olivier Donard, Laurent Ouerdane, and the other incredible scientists at the University of Pau for being incredible hosts and teaching me the secrets of successful LC-ICPMS. I would like to thank my undergraduate mentors, especially Sonbinh Nguyen, Andy Jacobson, and Thomas Meade for encouraging me to pursue my interests in research. I cannot imagine a better way to spend five years than by thinking about the mysteries of the ocean with such a passionate group of scientists. 
Thanks to the entire administration staff of WHOI and MIT who enabled me to survive the dayto-day of graduate school. A special thanks to Mary Zawoysky, Sheila Clifford, Mary Elliff, Leslie Adkins-Shellie, and Melody Abedinejad. Thanks to everyone in the academic programs office, including Julia Westwater, Marsha Gomes, Ronni Schwartz, Christine Charette, Valerie Caron, Lea Fraser, Meg Tivey and Jim Yoder. Thanks to Joe, John, and the WHOI security guards for essential transportation between Watson and the Village. I'd also like to thank Maria and Simone for keeping my lab and office space habitable, watering my plants, and even sharing snacks during those extra late nights.

To my mother, father, and sister Jocelyn, thank you for your endless encouragement and support. And to Kelsey, thanks for being a source of inspiration, motivation, and an amazing teammate in so many aspects of life.

Thanks to my many friends in EAPS and the Joint Program that provided tremendous support, encouragement, and welcome distractions, especially the FR Lillie crew (Melissa Moulton, Dan Amrhein, Katie Pitz, Christina Schulz, Alex Bergan, and Fulo), Kate French, Kyrstin Fornace, Nick Hawco, Deepak Cherian, Emily Estes, Sara Bosshart, Ben Mandler, Steve Messenger, Brian Green, Ruel Jerry, Yodit Tewelde, Arthur Olive, Becca Jackson, Christopher Kinsley, Christine Chen, Marie Giron, Aimee Gillespie, Sharon Newman, Simone Moos, Emily Matys, David Wang, Winn Johnson, Sarah Rosengard, Jordon Hemmingway, Sophie Chu, Andy Miller, Jaap Nienhuis, Elena Steponaitis, and many others. Thanks to my Watson lab mentors, Erin Bertrand, Katherine Mackey, Julia Gauglitz, and Kristen Whalen. I am also grateful to my Cambridge roommates Rishabh Jain, Pavan Nyama, Vikram Juneja, and Toby Mansuripur for making our living room one of the most enjoyable places to decompress. I am extremely fortunate to have such amazing and talented friends.

Finally, I would like to thank my funding sources. Thanks to the National Science Foundation Graduate Research Fellowship Program (NSF Award 0645960) for providing three years of funding. A special thanks to the National Science Foundation Chemical Oceanography Program (NSF awards OCE-01751733 and OCE-1356747) and the Gordon and Betty Moore Foundation for providing research and instrumentation funding that made this work possible. Additional support was provided by the Center for Microbial Oceanography: Research and Education (CMORE IF0424599) and the Simons Collaboration on Ocean Processes and Ecology (SCOPE). 
Chapter 1: Review of iron speciation in seawater

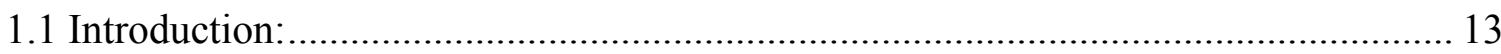

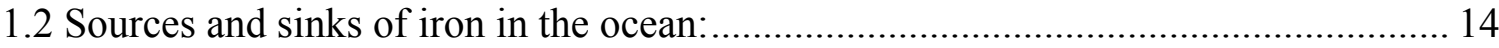

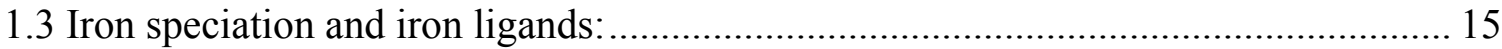

1.4 Ligands in global iron models: ............................................................................... 19

1.5 Sources and classes of iron ligands:...................................................................... 19

1.6 Identifying ligands in the marine environment:................................................ 23

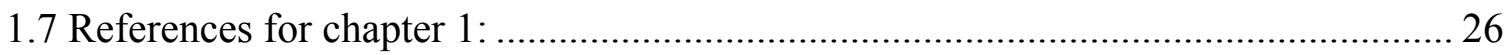

Chapter 2: Detection of iron ligands in seawater and marine cyanobacteria

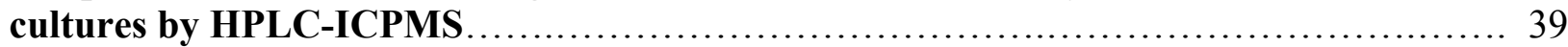

2.1 Introduction:

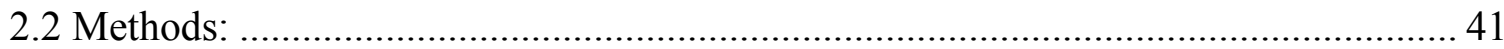

2.2.1 Materials, standards, and reagents: ........................................................... 41

2.2.2 Standard separation and isocratic standard injections: ................................ 42

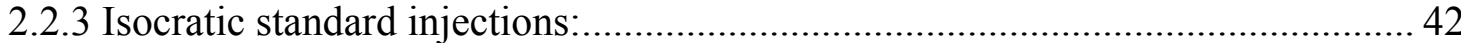

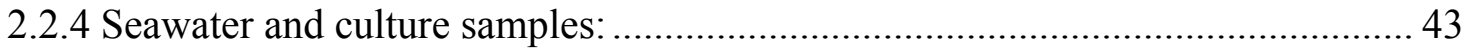

2.2.5 Solid phase organic extraction: ................................................................. 44

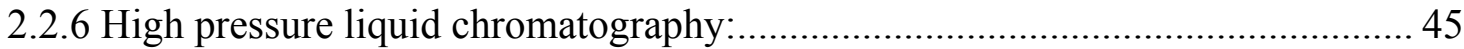

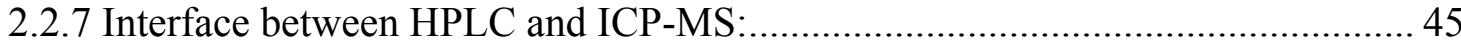

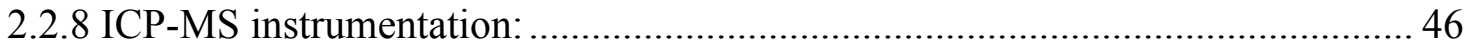

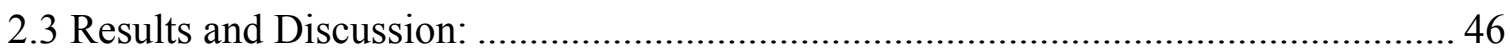

2.3.1 Separation of standard compounds: ........................................................... 46

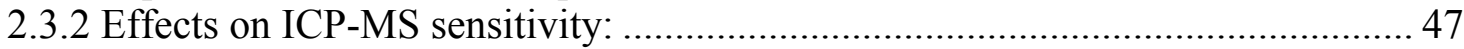

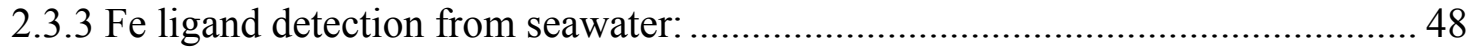

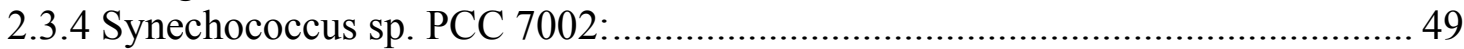

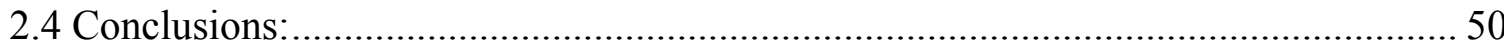

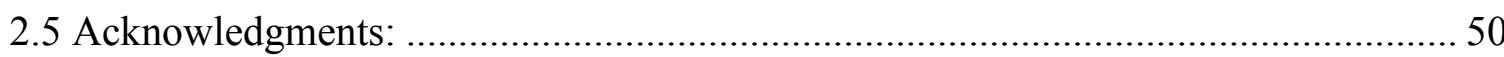

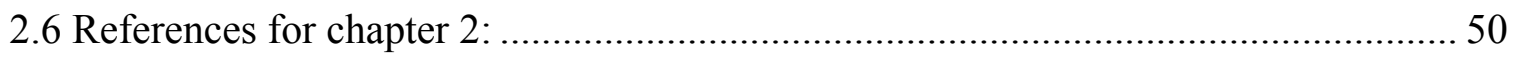

Appendix 2.1 Characterization of iron binding ligands from Prochlorococcus ....... 63

Chapter 3: An extended siderophore suite from Synechococcus sp. PCC 7002

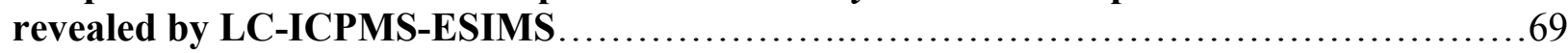

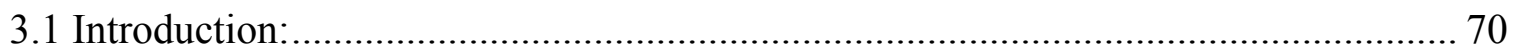

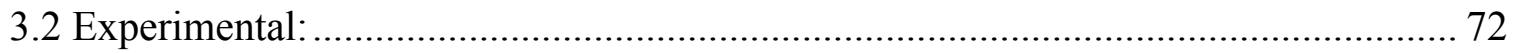

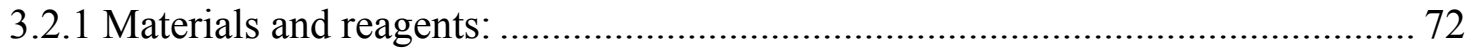




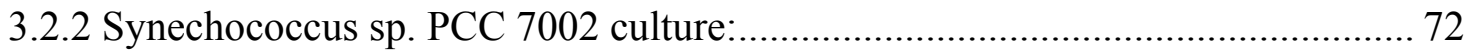

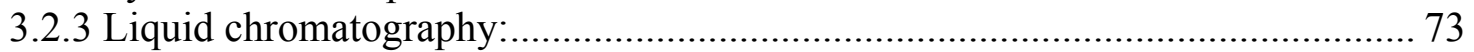

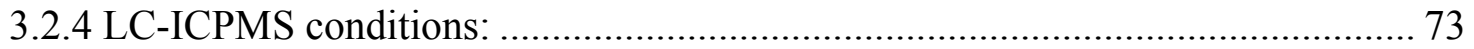

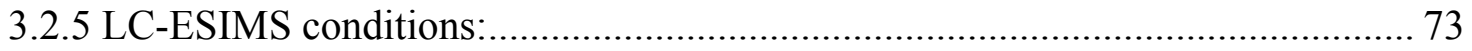

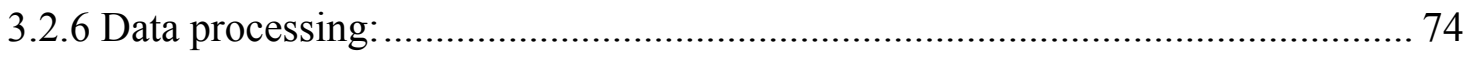

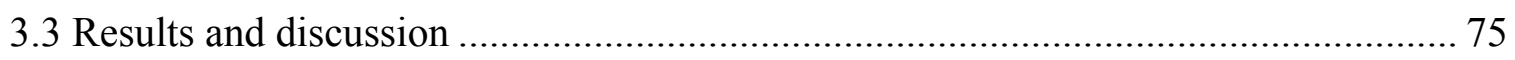

3.3.1 LC-ICP-MS:

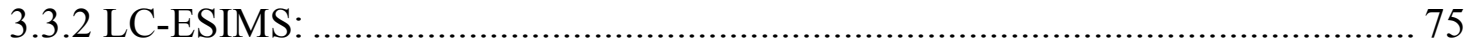

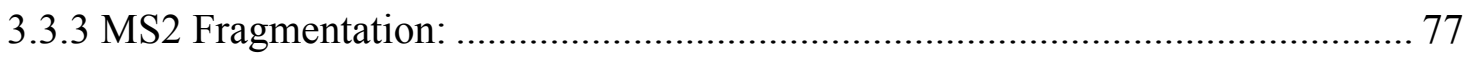

3.3.4 Connection to synechobactin biosynthesis: …………................................. 78

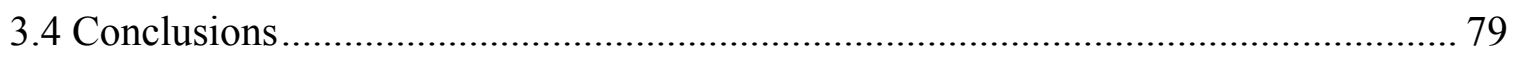

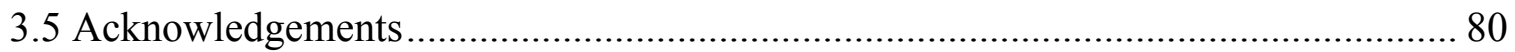

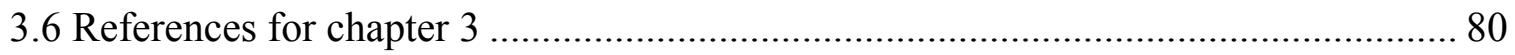

Appendix 3.1 Marinobactins from Costa Rica Upwelling Dome heterotrophs ...... 105

Chapter 4: Siderophore-based microbial adaptations to iron scarcity across

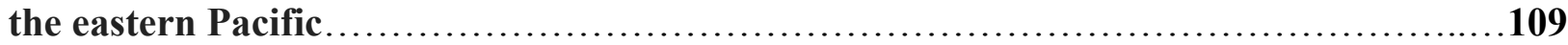

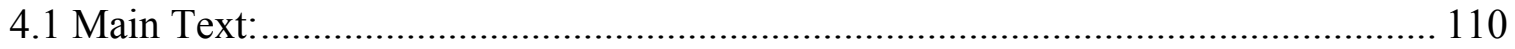

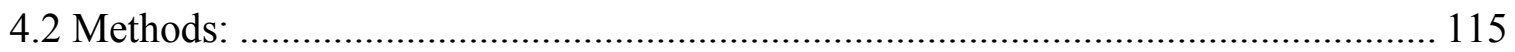

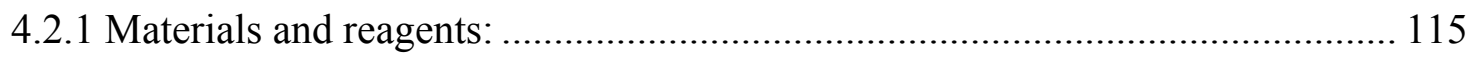

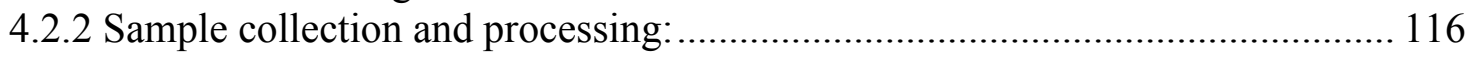

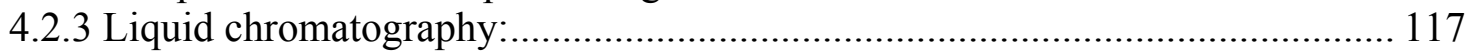

4.2.4 Inductively coupled plasma mass spectrometry (ICPMS):............................... 117

4.2.5 Electrospray ionization mass spectrometry (ESIMS) analysis: ......................... 118

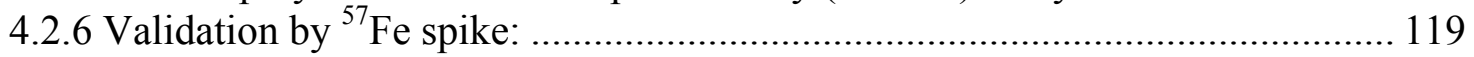

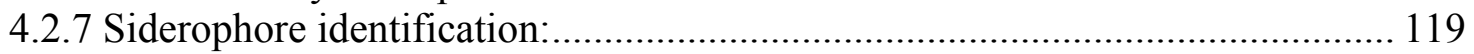

4.2.8 Vibrio laboratory culture: .......................................................................... 120

4.2.9 Tara Ocean catalog search: .................................................................. 120

4.3 Acknowledgments: .............................................................................................. 121

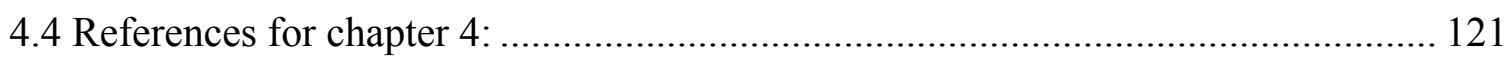

Appendix 4.1 Ni and Cu compounds across the Eastern Tropical South Pacific ... 139

Chapter 5: Diversity of metal ligands in the California Upwelling system: ...................... 143

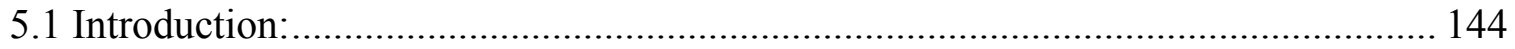

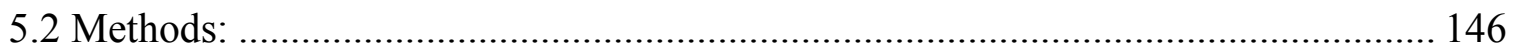

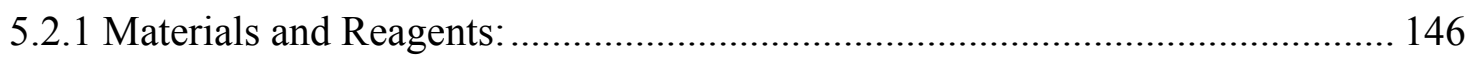

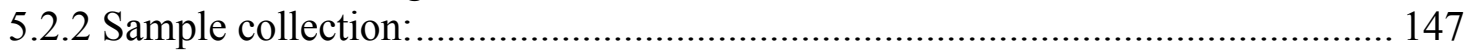

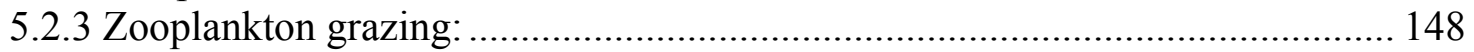

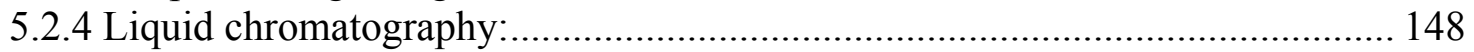

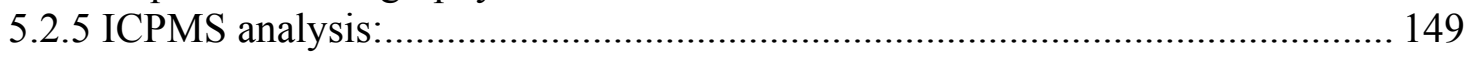




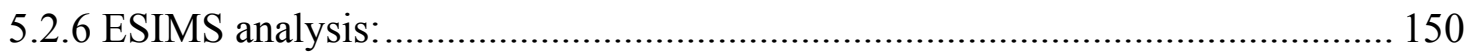

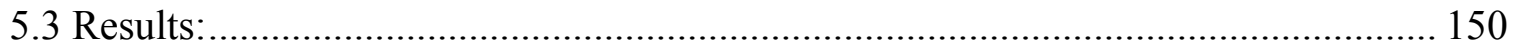

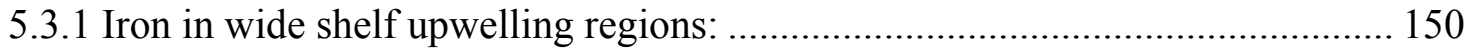

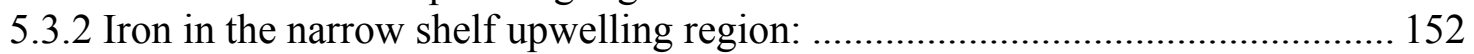

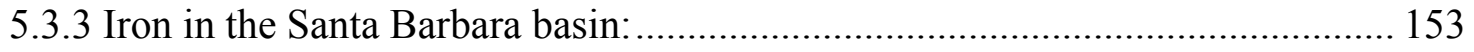

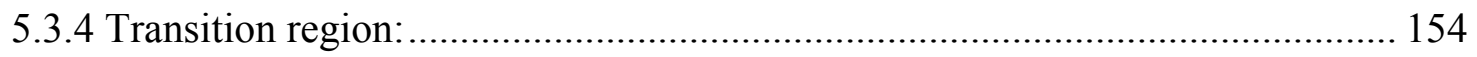

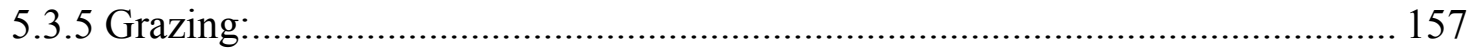

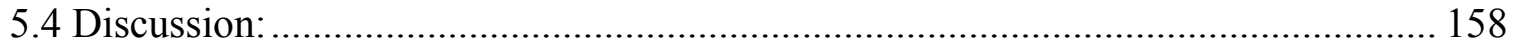

5.4.1 Unresolved carbon compounds from the deep: ............................................ 158

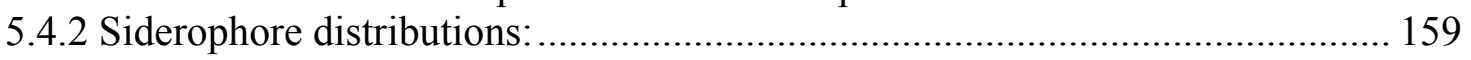

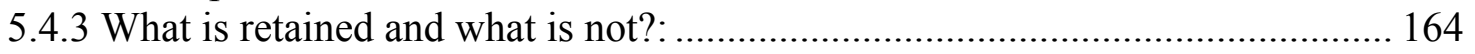

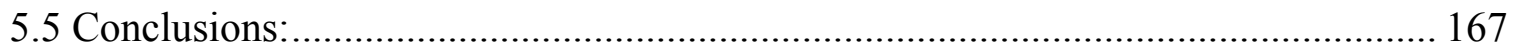

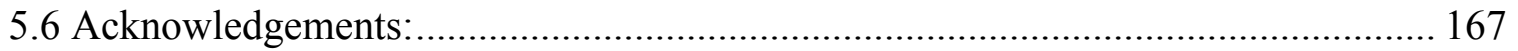

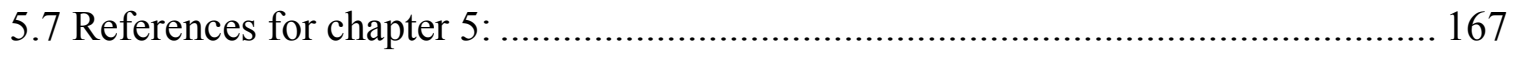

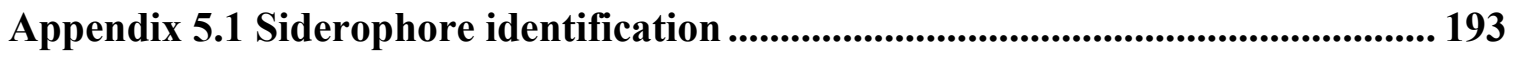

Appendix 5.2 Halogenated compounds in the upwelling community:....................... 200

\section{Chapter 6: Compound specific determination of ligand dissociation kinetics}

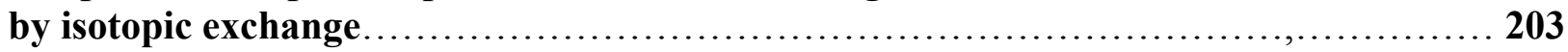

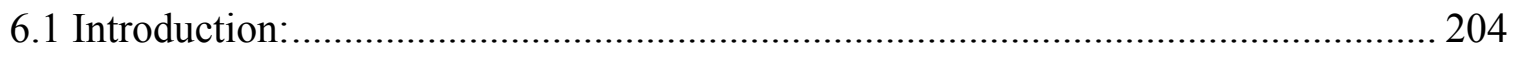

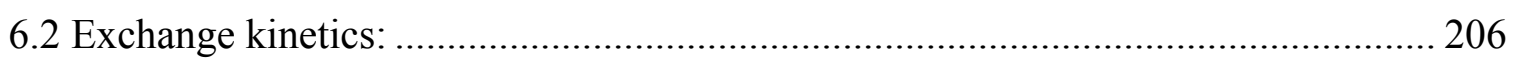

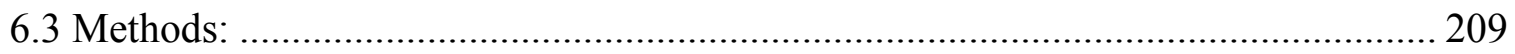

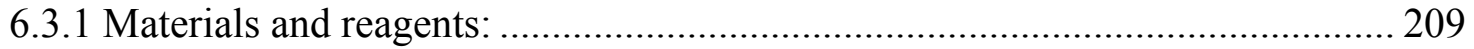

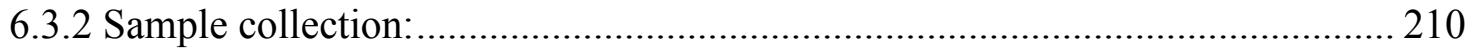

6.3.3 Fe exchange from model compounds under marine conditions: ....................... 210

6.3.4 Fe exchange of natural organic ligands: ......................................................... 211

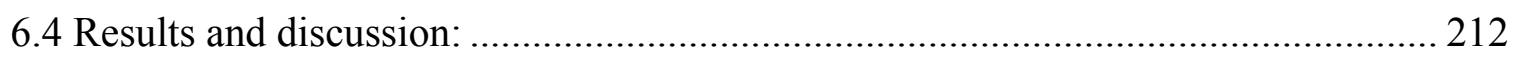

6.4.1 Fe exchange for model compounds under marine conditions: .......................... 212

6.4.2 Fe exchange of natural organic ligands: ....................................................... 215

6.5 Conclusions:

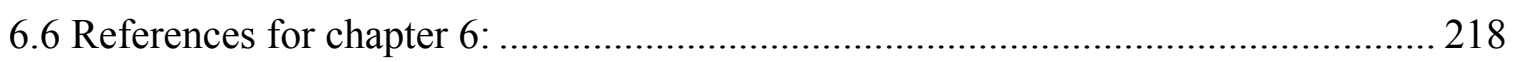

\section{Chapter 7: Conclusions and future directions}

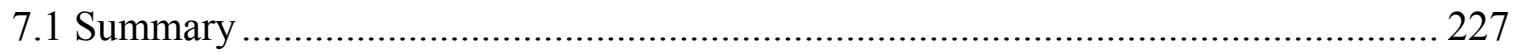

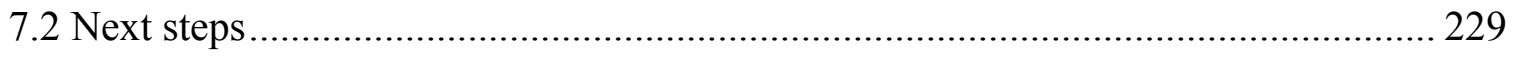




\section{List of Figures:}

\section{Chapter 1}

Figure 1.1 Representative profile of dissolved Fe and Fe ligands

\section{Chapter 2}

Figure 2.1 Schematic of HPLC-ICPMS

Figure 2.2 Separation of standards by HPLC-ICP-MS

Figure 2.3: Effect of solvent composition on ICP-MS signal

Figure 2.4: Separation of organic extract from seawater

Figure 2.5: Separation of organic extract from a Synechococcus sp. PCC 7002 culture.

Figure A2.1 Detection of Fe Ligands from Prochlorococcus strains

Figure A2.2 Prochlorococcus ligands at multiple [Fe]

Figure A2.3: Growth curves for Prochlorococcus strains at multiple [Fe]

\section{Chapter 3}

Figure 3.1 Workflow for unknown iron compound identification

Figure 3.2 Chemical structure of the synechobactins and related compounds.

Figure 3.3 LC-MS chromatograms of Synechoccoccus sp. PCC 7002 media extract

Figure 3.4 Positive mode MS2 spectra of the six identified Fe containing compounds.

Figure 3.5 Characteristic fragmentation patterns of Fe-synechobactins

Figure SI-1 Chromatograms and mass spectra from the major Fe features

Figure SI-2 Apo and positive mode MS2 spectra for synechobactins

Figure SI-3 EIC and MS2 spectra of interfering ions

Figure SI-4 Extracted ion chromatograms of synechobactin $\mathrm{C}_{9}$ and synechobactin $\mathrm{C}_{16}$

Figure SI-5 Schizokinen production by Synechococcus sp. PCC 7002.

Figure A3.1 Marinobactins identified from alteromonas 2E5

\section{Chapter 4}

Figure 4.1 US GEOTRACES EPZT cruise track

Figure 4.2 Siderophore identification by LCMS

Figure 4.3 Geographic locations of amphibactin biosynthesis genes.

Figure S4. $1{ }^{56} \mathrm{Fe}$ LC-ICPMS chromatograms for EPZT

Figure S4.2 Ferrioxamine B characterization

Figure S4.3 Ampibactin D characterization

Figure S4.4 Unknown compound $(959 \mathrm{~m} / \mathrm{z})$ characterization

Figure S4.5 Unknown compound $(709 \mathrm{~m} / \mathrm{z})$ characterization

Figure $\mathrm{S} 4.6{ }^{57} \mathrm{Fe}$ validation of siderophore masses for unknown

Figure S4.7 Phylogenetic tree of amphibactin synthesis genes

Figure S4.8 Depth distributions of amphibactin synthesis genes

Figure A4.1 ${ }^{60} \mathrm{Ni}^{63} \mathrm{Cu}$ LC-ICPMS chromatograms from EPZT

\section{Chapter 5}

Figure 5.1 Map of California coast and station sampling locations

Figure 5.2 Station 2, Wide shelf upwelling region

Figure 5.3 Station 28, Narrow shelf upwelling region 
Figure 5.4 Station 29 - Santa Barbara basin station

Figure 5.5 Transect across CCS eddies

Figure 5.6 Iron ligands across CCS eddies

Figure 5.7: Iron ligands across nearshore eddy

Figure 5.8 Iron ligands from zooplankton grazing

Figure 5.9 SPE extraction efficiency for iron

Figure 5.10 Extraction comparison

Figure 5.11: SPE resin comparison

Figure A5.1-1 Identification of synechobactin A (c12)

Figure A5.1-2 Identification of synechobactin c14

Figure A5.1-3 Identification of Amphibactin I

Figure A5.1-4 Identification of Amphibactin E

Figure A5.1-5 Identification of Amphibactin S

Figure A5.1-6 Identification of new siderophore (m/z 709)

Figure A5.1-7 Putative identification of ferrioxamine B

Figure A5.2-1: LC-ICPMS chromatograms of halogenated compounds

\section{Chapter 6}

Figure 6.1 Siderophore exchange in seawater

Figure 6.2 Siderophore exchange in water

Figure 6.3 Isotopic ratios of ferrichrome and ferrioxamine E over time

Figure 6.4 Isotopic exchange of natural ligands 


\section{List of Tables:}

\section{Chapter 2:}

Table 2.1: Operating conditions for HPLC-ICP-MS analysis.

Table 2.2: Chromatography and detection of standards

\section{Chapter 3:}

Table 3.1: Siderophores from Synechococcus sp. PCC 7002

Table SI-1: Complete list of curated isotope pairs identified by algorithm

\section{Chapter 4:}

Table S4.1: Iron compounds detected by LC-ICPMS-ESIMS

Table S4.2: EPZT sample list

Table A4-1: Ni and $\mathrm{Cu}$ compounds identified across the GEOTRACES EPZT transect

\section{Chapter 5:}

Table 5.1: Identification of iron binding compounds in CCS seawater

Table 5.2: Identification of Fe compounds in the IRNBRU grazing experiment

Table 5.3: CCS sample list

Table 5.4: CCS sample ligand description

Table A5.2-1: Brominated compounds detected within the wide shelf surface waters

\section{Chapter 6:}

Table 6.1: Dissociation rates of siderophores from this study 


\section{Chapter 1: Review of iron speciation in seawater}

\subsection{Introduction:}

Iron $(\mathrm{Fe})$ is an essential micronutrient for nearly every organism in the ocean. It is at the heart of cellular machinery that carries out photosynthesis, oxic respiration, nitrate assimilation and nitrogen fixation [Bruland et al., 1991; Morel and Price, 2003; Morel et al., 2003; Leão et al., 2007]. Cells must assimilate iron from their environment in order to meet their quotas, which typically range from 0.2-20 mM Fe per M phosphorus in marine plankton (P) [Ho et al., 2003; Twining and Baines, 2011]. Yet Fe is scarcely soluble in oxic waters. As a result, high concentrations of $\mathrm{N}$ and $\mathrm{P}$ persist in large regions of the surface ocean due to an insufficient supply of Fe, particularly where its input from dust deposition and upwelling are low [Boyd and Ellwood, 2010].

In the late 1980's, Martin and colleagues suggested that Fe is a major limiting nutrient for primary productivity in many regions of the ocean characterized by high nitrate and phosphate stocks [Martin and Fitzwater, 1988; Martin et al., 1990, 1991]. Shortly thereafter, their hypothesis was confirmed by a number of mesoscale Fe fertilization experiments that resulted in phytoplankton blooms, primarily dominated by fast growing diatoms [De Baar et al., 2005; Boyd et al., 2007]. These results were the first to demonstrate the biogeochemical importance of Fe in shaping the marine community composition and productivity [Martin et al., 1994; De Baar et al., 2005; Jickells et al., 2005; Boyd et al., 2007]. Modeling results suggest that low Fe concentrations limit primary productivity in over one third of the global ocean [Moore et al., 2002, 2004]. Furthermore, Fe exerts a primary control over nitrogen fixation, and thus has important consequences for the nitrate inventory of the ocean [Moore and Doney, 2007].

Since the recognition of iron's role as a micronutrient, it has been a central goal of chemical oceanography to develop better constraints on the sources and sinks of Fe across the ocean and incorporate this information into biogeochemical models of community composition and carbon/nutrient cycling. One of the greatest sources of uncertainty in these models has been the chemical speciation of Fe. Organic ligands are thought to dominate Fe speciation, yet despite 
knowledge of their existence for over 20 years, the chemical structure of these compounds has remained poorly constrained. This thesis describes the development and application of novel methods for determining the chemical identity of $\mathrm{Fe}$ binding compounds in natural seawater.

\subsection{Sources and sinks of iron in the ocean:}

Measurements of $\mathrm{Fe}$ are typically partitioned into filterable size classes, which are operationally defined as soluble (which passes through a filter, typically $<0.02 \mu \mathrm{m})$, colloidal $(0.02 \mu \mathrm{m}-$ $0.2 \mu \mathrm{m}$ ), dissolved $(<0.2 \mu \mathrm{m})$, and particulate (typically $>0.2 \mu \mathrm{m}$ ) forms [Boyd and Ellwood, 2010]. The soluble class roughly approximates the concentration of Fe in truly dissolved organic ligands or inorganic species. Colloidal Fe includes organic aggregates, viruses, and small iron oxhydroxides, Particulate $\mathrm{Fe}$ includes detrital/biogenic particles and biomass. These forms undergo different rates of settling, scavenging, and biological uptake, and many processes govern dynamic exchange between them (described below).

The primary sources and sinks of $\mathrm{Fe}$ have been determined by observing the distribution of $\mathrm{Fe}$ in dissolved/particulate phases and their isotopic compositions. Atmospheric deposition is considered the primary source to the surface ocean, contributing an estimated $0.5-2 \times 10^{12} \mathrm{~g}$ Fe/year [Ussher et al., 2004; Moore and Braucher, 2008; Worsfold et al., 2014] and accounting for $71-87 \%$ of dissolved Fe in the North Atlantic Ocean [Conway and John, 2014]. Additional Fe enters the ocean from oxygenated or reducing sediments [Lohan and Bruland, 2008; Bundy et al., 2015a], riverine inputs, and hydrothermal vents [Yücel et al., 2011; Hawkes et al., 2013; Saito et al., 2013; Fitzsimmons et al., 2014a]. The highest concentrations of Fe are typically found near these sources, in some cases exceeding $4 \mathrm{nM}$ [Tagliabue et al., 2012]. Oceanographic distributions of dissolved $\mathrm{Fe}$ are heavily influenced by both biological uptake and scavenging processes. Deep ocean concentrations of dissolved Fe are variable, generally ranging from 0.4 to $0.8 \mathrm{nM}$ and indicating a short residence time for Fe relative to ocean overturning circulation [Conway and John, 2014; Resing et al., 2015]. These concentrations reflect the balance of Fe addition through the remineralization of Fe-containing biomass at depth along with removal by scavenging onto sinking particles. Scavenging processes (adsorption, aggregation, and 
precipitation) account for the loss of Fe from the system in particulate form and removal by burial in sediments [Bruland et al., 2013].

In the euphotic surface ocean $(<200 \mathrm{~m})$, Fe concentrations are highly dynamic and the residence time of Fe is extremely short ( $<1 \mathrm{yr}$ ) [Boyle et al., 2005; Fitzsimmons et al., 2015a; Hayes et al., 2015]. The major sources of $\mathrm{Fe}$ to the sunlit ocean are atmospheric deposition or upwelling of Fe-rich deep waters. Intense biological utilization depletes dissolved Fe concentrations to below $0.2 \mathrm{nM}$ throughout most of the surface ocean.

Colloidal Fe generally accounts for a significant fraction of dissolved Fe, ranging from 30-60\% in most of the deep ocean, with higher values observed near major Fe sources such as coastal margins and hydrothermal vents [Boyd and Ellwood, 2010; Fitzsimmons et al., 2015b]. In the surface ocean, colloidal Fe also makes up a significant fraction of dissolved Fe in regions with high dust inputs (for example, $80 \pm 7 \%$ in the North Atlantic) compared with smaller percentages in low-dust regions (64 $\pm 18 \%$ in the North Pacific Subtropical Gyre) [Fitzsimmons et al., 2015b].

\subsection{Iron speciation and iron ligands:}

Iron(III) is the thermodynamically favored form of $\mathrm{Fe}$ in oxic waters at seawater $\mathrm{pH}$ (8.2), and the dominant inorganic $\mathrm{Fe}$ species are $\mathrm{Fe}(\mathrm{OH})^{2+}, \mathrm{Fe}(\mathrm{OH})_{2}{ }^{+}$, and $\mathrm{Fe}(\mathrm{OH})_{3}{ }^{0}$ [Turner et al., 1981]. Previous studies have demonstrated that the solubility of $\mathrm{Fe}(\mathrm{III})$ in artificial seawater is extremely low ( $\sim 10 \mathrm{pM})$, due to precipitation as iron oxyhydroxides [Liu and Millero, 2002; Schlosser and Croot, 2009]. In natural seawater, however, Fe solubility typically exceeds 0.5 $\mathrm{nM}$, although the solubility is reduced after UV irradiation [Wu and Luther, 1995]. The high solubility of dissolved iron in natural seawater has been attributed to the stabilization of $\mathrm{Fe}$ (III) by naturally occurring organic ligands (Fig. 1.1). While these ligands maintain an elevated standing stock of $\mathrm{Fe}$ in the ocean, they also impact the ability of marine organisms to acquire that Fe [Hutchins et al., 1999]. The ability of marine microorganisms to access this organic pool of Fe offers a distinct competitive advantage. Thus, determining the organic speciation of dissolved $\mathrm{Fe}$ in the ocean and the Fe uptake strategies of microorganisms are critical for developing global 
models that can accurately predict the distribution of microbial species and primary productivity in Fe-deficient regions.

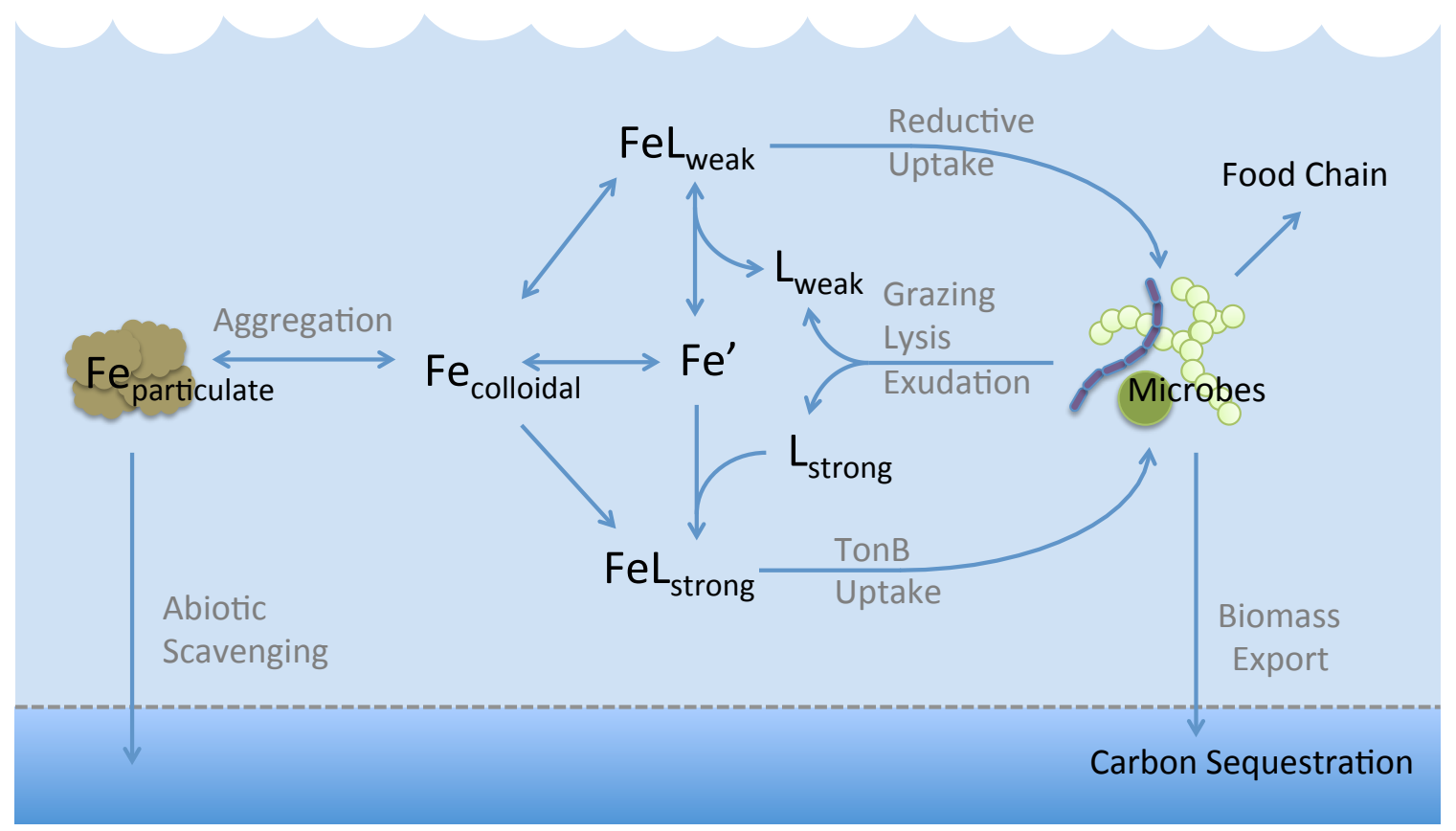

Figure 1.1: Schematic of iron cycling in the ocean. Strong and weak organic ligands stabilize soluble iron and impact iron acquisition by the microbial community.

The first measurements of the concentrations and binding strengths of organic Fe binding ligands in seawater were achieved with competitive ligand-exchange cathodic stripping voltammetry (CLE-CSV) measurements [Gledhill and van den Berg, 1994, 1995; Rue and Bruland, 1995; Gledhill and Buck, 2012]. Excess (i.e. unbound) ligand concentrations are common in most of the ocean except for near hydrothermal or benthic boundary Fe sources. These ligands are typically highest and most variable in the upper water column. Data from CLE-CSV measurements are often modeled as two classes of organic Fe ligands [Rue and Bruland, 1995, 1997; Cullen et al., 2006; Buck and Bruland, 2007; Buck et al., 2007]. When this is done, a weak binding class (L2) is generally present throughout the water column at concentrations of $\sim 1-2$ $\mathrm{nM}$, while a strong binding class (L1) is found predominantly in the upper water column at concentrations of up to $0.5 \mathrm{nM}$ (Fig. 1.2). 


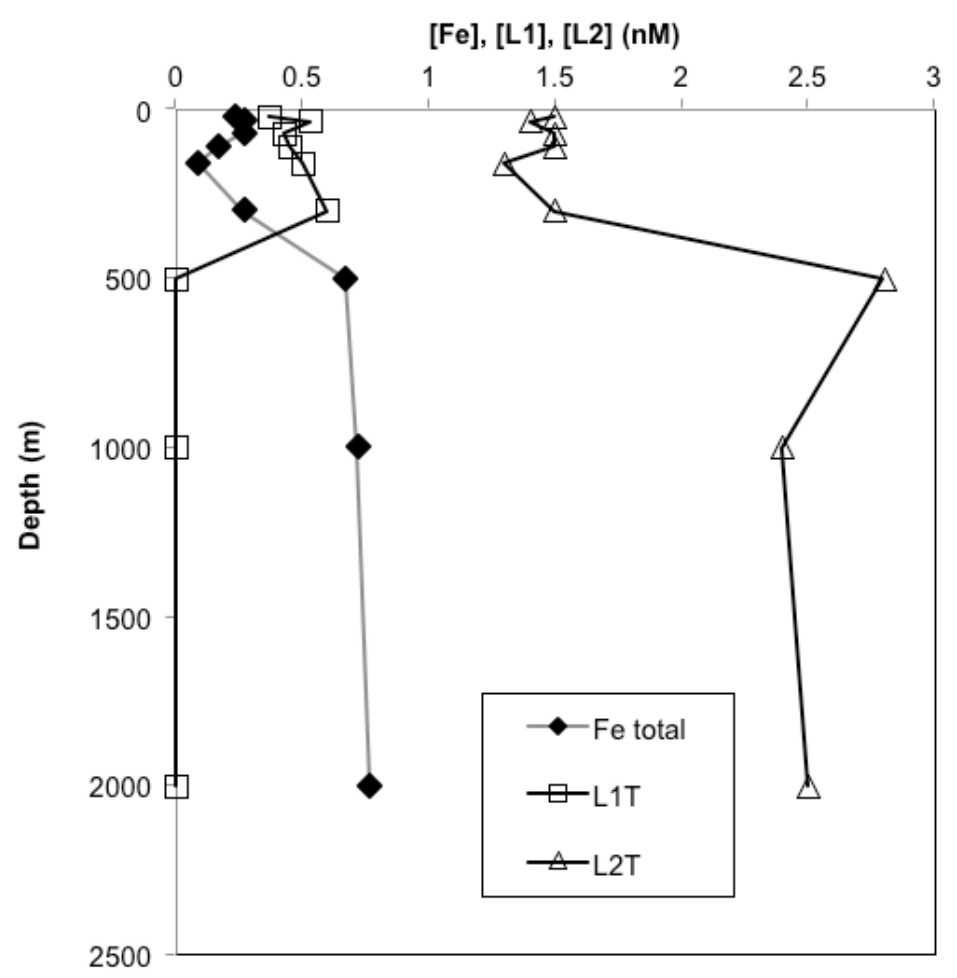

Figure 1.2: Representative depth profiles of total dissolved $\mathrm{Fe}[\mathrm{Fe}]_{\mathrm{T}}$, total strong ligand $\left[\mathrm{L}_{1}\right]_{\mathrm{T}}$, and total weak ligand $\left[\mathrm{L}_{2}\right]_{\mathrm{T}}$ in the central North Pacific at station ALOHA [Rue and Bruland, 1995].

The highest concentrations of excess ligand are generally found in the chlorophyll maxima and are predominantly in the soluble $(<0.02 \mu \mathrm{m})$ size class [Wu et al., 2001; Cullen et al., 2006; Boye et al., 2010; Thuróczy et al., 2010]. These observations have led to speculation that strong Fe ligands are biologically produced [Rue and Bruland, 1995]. More recently, competitive ligandexchange experiments with multiple analytical windows have been used to identify additional weaker ligand classes in coastal environments that were associated with terrestrial inputs. Overall, these results have suggested that $\mathrm{Fe}$ is predominantly complexed ( $>99 \%)$ by organic ligands in the ocean, although the identity, source, and fate of these ligands is still unknown.

The cycling of $\mathrm{Fe}$ between organic and inorganic phases is governed by both biological and abiotic factors. As an adaptation to low environmental $\mathrm{Fe}$ concentrations, microbes have 
developed a wide array of Fe uptake pathways [Morel et al., 2008]. The simplest are ABC transporters that actively import free $\mathrm{Fe}$ (III) or Fe(II) into cells. Other organisms, such as diatoms, combine an Fe reduction step at the cell surface followed by Fe(II) uptake [Maldonado and Price, 2001]. Still others, including many copiotrophic bacteria, utilize tonB dependent transporters to bring Fe-organic complexes directly into the cell, with varying levels of compound specificity [Ratledge and Dover, 2000; Wandersman and Delepelaire, 2004; Braun and Hantke, 2013]. Inside of the cell, Fe is primarily incorporated into heme cofactors, enzymes, and storage/transport proteins. These forms of Fe are released from cells upon death by viral lysis or grazing, and are either recycled back into the dissolved pool or packaged into sinking particles (e.g. fecal pellets).

In the surface ocean, direct or indirect photochemical oxidation is a potentially important process that supplies free Fe(II) species [Miller and Zepp, 1995; Hansard et al., 2009]. This process is intimately linked to organic matter speciation - some organic compounds can increase the lability of Fe(III) to photochemical reduction while others inhibit it [Barbeau, 2006; Hopkinson and Barbeau, 2007]. The reoxidation of Fe(II) generally occurs rapidly (within minutes) in seawater, although $\mathrm{Fe}(\mathrm{III})$ binding organic ligands can catalyze the oxidation [Roy et al., 2008; Roy and Wells, 2011], while Fe(II) stabilizing organic ligands can decrease oxidation rates [Kieber et al., 2005; Willey et al., 2008]. Fe(II) is far more soluble than Fe(III), and under anoxic conditions it can accumulate to $\mathrm{mM}$ concentrations (such as anoxic sediments) [Lohan and Bruland, 2008].

Abiotic scavenging processes also have a major impact on $\mathrm{Fe}$ cycling, similar to those that control thorium (Th). The conceptual model for removal by scavenging involves reversible adsorption to colloidal size particles and aggregation of colloids into larger sinking particles. These colloidal forms are poorly constrained and may include inorganic (oxide or oxyhydroxide phases) or organic phases (incorporation into humic substances, large acidic polysaccharides, or vesicles/viruses) [Wells and Goldberg, 1992; Wells et al., 1994]. Iron scavenging models have ranged in complexity from simple first order net loss rates to explicit representations of adsorption, desorption, aggregation, and sinking [Moore and Braucher, 2008]. Several studies have suggested that colloidal Fe in the surface ocean (potentially dust derived) is inert to ligand 
exchange, at least over short timescales [Cullen et al., 2006; Fitzsimmons et al., 2014b], while Fe in the deep ocean appears to partition between colloidal and soluble phases, perhaps indicating a constant exchange between these pools following remineralization [Fitzsimmons et al., 2015a, 2015b]. These differences may be attributed to either slow kinetics of Fe exchange between phases or to variability in ligand/colloid chemical properties. Improved characterization of the chemical nature of both soluble organic Fe binding ligands and colloidal Fe will shed light on these processes.

\subsection{Ligands in global iron models:}

Iron ligands are a key aspect of most biogeochemical models, and ligand sources/sinks are essential parameters that remain poorly constrained. Most ocean general circulation and biogeochemistry models assume a single ligand class with uniform concentration (typically 0.6 $\mathrm{nM}$ ). Other models have tied ligand concentration to dissolved organic carbon concentrations [Tagliabue and Völker, 2011], although this does not accurately reflect the distribution of ligands in the marine environment [Boyd and Tagliabue, 2015]. More recently, models have implemented prognostic ligands based on remineralization rates or apparent oxygen utilization [Misumi et al., 2013; Völker and Tagliabue, 2014]. To more accurately account for faster decay of labile ligands, ligand degradation rates have been tied to ligand concentration [Völker and Tagliabue, 2014]. All of these models represent ligands as a single compound class. Ecosystem models have also implemented iron ligands, typically as a fixed value, and assume that all $\mathrm{Fe}$ is equally available to all organisms [Follows et al., 2007; Dutkiewicz et al., 2012]. More sophisticated models that capture the dynamics of iron ligand production in the surface ocean require better constraints on the provenance of ligands and their fate/bioavailability.

\subsection{Sources and classes of iron ligands:}

The biogeochemical importance of Fe has sparked an interest in characterizing Fe binding organic compounds in seawater and determining the impact that these compounds have on microbial uptake. In terms of their chemical composition, marine ligands can be broadly 
classified into two groups that differ by their chemical characteristics and formation pathway (Hertkorn et al., 2007). The first group is made up of chemically well-defined, discrete molecules that are synthesized by cellular machinery encoded in microbial genomes. These compounds often regulate Fe uptake and metabolism, and bind Fe very strongly and specifically (Morel et al., 2003; Wandersman and Delepelaire, 2004). Ligands that fall within this group can be resolved by chromatography and structurally elucidated. When organic matter is altered by nondirected biotic and abiotic process (e.g., redox reactions and photochemistry), it can become part of the second group of compounds: an ill-defined complex mixture of compounds with similar characteristics to humic substances. Because of their extreme chemical diversity, a fraction of these molecules contains functional groups that complex trace metals, sometimes with very strong stability constants (Hertkorn et al., 2006; Yang and Van den Berg, 2009). Examples of both groups have been detected in seawater (Mawji et al., 2008; Laglera et al., 2009, 2011), but their chemical variability and relative contribution to the ligand pool has not been investigated.

Of the small well-defined biomolecules that bind $\mathrm{Fe}$, siderophores have garnered the most interest from the marine community due to the similarity between siderophore Fe binding strengths and the binding strengths of strong marine ligands. Siderophores are strong Fe-binding organic chelators that are exuded by microbes under conditions of Fe scarcity to bind Fe. These compounds compete with ambient Fe ligands in the surrounding environment and complex it in a form that can be recognized and taken up by membrane bound receptors [Butler, 1998; Wandersman and Delepelaire, 2004; Hopkinson and Morel, 2009; Sandy and Butler, 2009].

Many structurally diverse siderophores have been identified from marine bacteria in culture (see Fig. 1.3). Often, they are amphiphilic compounds that contain a fatty acid tail linked to a peptidic head group. These compounds include the marinobactins, amphibactins, moanachelins, aquachelins, loihichelins, all of which bind Fe with three hydroxamate groups [Kanoh et al., 2003; Holt et al., 2005; Martinez and Butler, 2007; Homann et al., 2009a; Zhang et al., 2009; Vraspir et al., 2011; Gauglitz and Butler, 2013]. Other marine amphiphilic siderophores include synechobactins and ochrobactins, which are mixed-mode compounds containing both citrate and two hydroxamate groups [Ito and Butler, 2005; Martin et al., 2006]. These amphiphilic compounds are able to partition into cell membranes, potentially providing a competitive 
advantage in dilute aquatic environments where diffusive losses may be substantial $[X u$ et al., 2002; Martinez et al., 2003; Martin et al., 2006; Martinez and Butler, 2007]. Numerous catechol based siderophores have also been characterized from laboratory cultures of marine bacteria, including petrobactin (which also contains citrate), alterobactins, pseudoalterobactins, amonabactin, vancrobactin, and anguibactin [Telford and Raymond, 1997; Homann et al., 2009b; Sandy et al., 2010]. The well-known group of tris-hydroxamate siderophores ferrioxamines B, E and $\mathrm{G}$ have also been observed from marine bacteria, as have the citrate-based siderophores aerobactin (a di-hydroxamate siderophore) and vibrioferrin [Martinez et al., 2001; Mawji et al., 2011; Cordero et al., 2012].
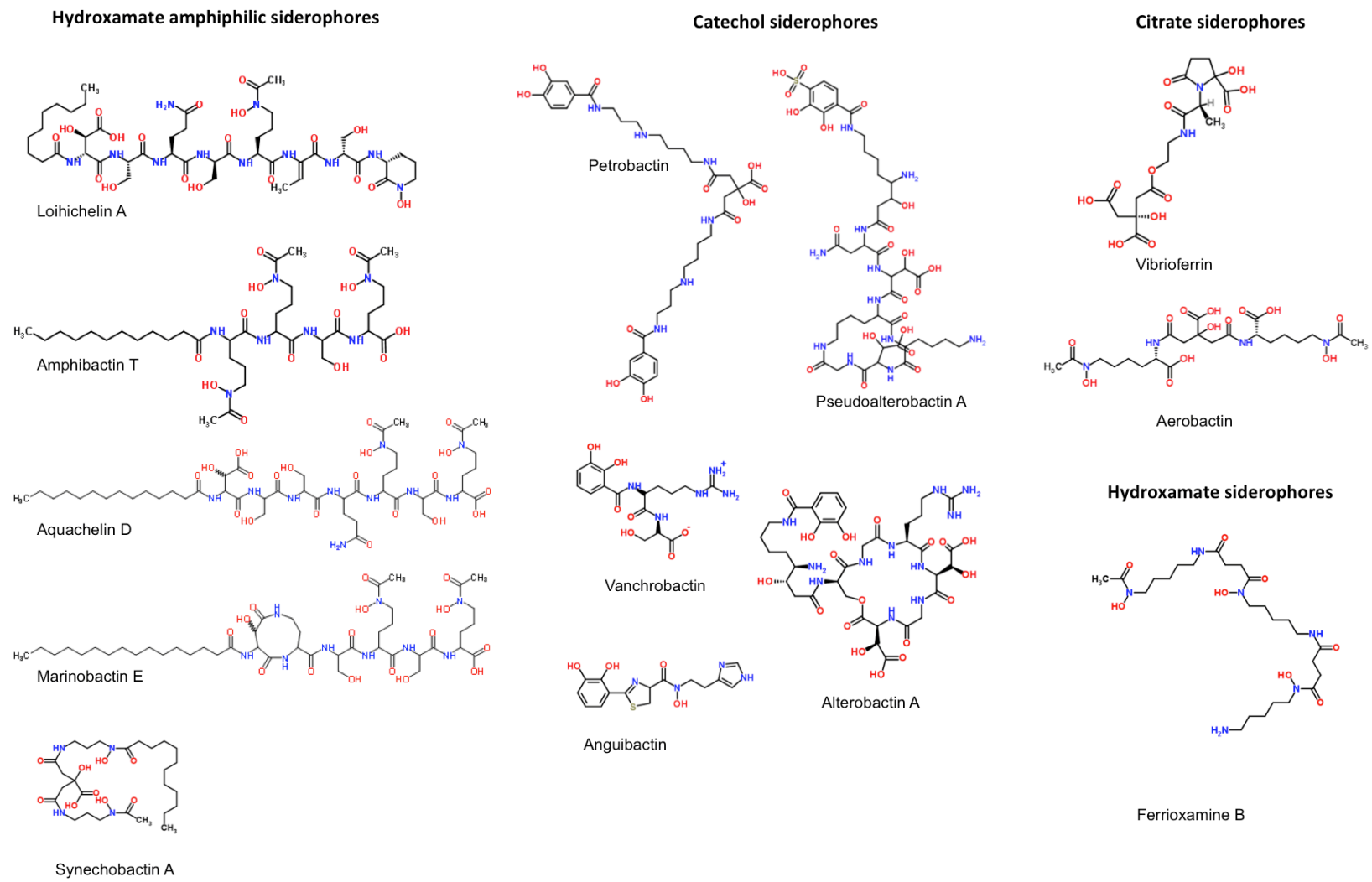

Hydroxamate siderophores
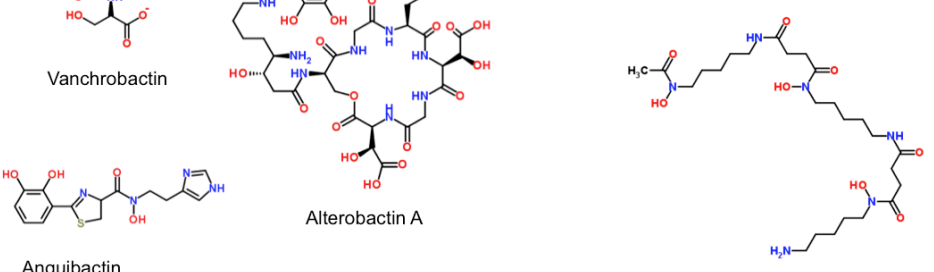

Anguibactin

Ferrioxamine B

Figure 1.3: Chemical structure of siderophores with different iron binding functional groups isolated from marine bacterial cultures (after Sandy and Butler 2009). 
The production of siderophores is relatively common among fast growing marine heterotrophs, but is thought to be rare in marine phytoplankton based on genomic screening. However, some 'cheater' organisms are able to utilize siderophores by expressing genes for siderophore acquisition without producing the compounds themselves [Cordero et al., 2012; Malmstrom et al., 2013]. Putative siderophore transport genes have been identified in single cell genomes of Prochlorococcus from the HL III and HL IV clades living in low Fe environments, suggesting that siderophore-mediated uptake may be important for photoautotrophs in these regions [Malmstrom et al., 2013]. Furthermore, metagenomic surveys of genes involved in siderophore uptake appear to be common in low Fe regions of the ocean, although the corresponding siderophore synthesis genes have not been identified [Hopkinson and Barbeau, 2012; Toulza et al., 2012]. In other cases, microbes produce superoxides or rely on photochemistry to convert siderophore-bound Fe(III) into free Fe(II), which can be taken up [Barbeau et al., 2001, 2003; Kustka et al., 2005; Shaked et al., 2005; Salmon et al., 2006; Kranzler et al., 2011; Roe et al., 2012]. Others ingest Fe-rich bacteria to fulfill their quotas [Maranger et al., 1998].

Porphyrins, such as hemes, are another potential component of the soluble Fe pool. These compounds are cofactors in numerous enzymes that absorb light, transfer electrons, and bind to oxygen [Hogle et al., 2014]. Picomolar concentrations of heme have been detected in particulate material [Gledhill, 2007, 2014], and they are likely released into the water column by grazing. Some bacteria, such as Roseobacter possess heme uptake systems similar to those used for siderophores, which allows them to directly access heme-bound Fe released from dead cells [Roe et al., 2013]. This strategy is likely important for recycling $\mathrm{Fe}$ in the open ocean.

The importance of structurally ill-defined ligands to the ligand pool has emerged in recent years. Some of the main components of marine dissolved organic carbon are carboxylic rich aliphatic compounds that result from the degradation of organic matter. These compounds are thought to be a collection of primarily small $(<600 \mathrm{Da})$ and extremely diverse chemical isomers [Hertkorn et al., 2006, 2007]. Thanks to the extreme structural variability of these compounds, a portion of these compounds may have strong affinity for metals by chance rather than by design. Other studies have characterized 'humic-like' organic ligands that can be detected using anodic stripping voltammetry to measure the reduction current produced by Fe bound to marine ligands 
[Laglera et al., 2007; Laglera and van den Berg, 2009]. These studies have suggested that humic substances may complex the majority of dissolved $\mathrm{Fe}$ in the coastal and deep ocean [Laglera et al., 2011; Abualhaija et al., 2015; Bundy et al., 2015b]. However, other compounds in addition to marine or terrestrial humic substances may also be electrochemically active and thus the nature of these compounds remains unclear.

Exopolysaccharides have also been suggested to play a major role in solubilizing $\mathrm{Fe}$ in the ocean and impacting its bioavailability. These macromolecules are important for biofilm formation and bacterial attachment, and often incorporate acidic sugars that are capable of complexing dissolved Fe. Studies by Hassler et al. have demonstrated that acidic exopolysaccharides reduce Fe binding to strong ligands and enhance bioavailability to marine phytoplankton, suggesting that polysaccharides may play a major role in Fe solubility and uptake in the surface ocean [Hassler et al., 2011a, 2011b, 2014; Norman et al., 2015], where exopolysaccharides may comprise up roughly $25 \%$ of the dissolved organic carbon [Repeta, 2015].

These intriguing thoughts on the potential chemical composition of marine ligands require verification from experiments that directly characterize the chemical structure of metal binding compounds in the marine environment. As a first step, the abundance, sources and sinks of biomolecules such as siderophores and heme must be determined.

\subsection{Identifying ligands in the marine environment:}

To inform biogeochemical models of Fe cycling, it is necessary to connect the laboratory-based characterization and environmental studies on 'model compounds' to Fe cycling in the environment, and this requires sensitive techniques for identifying the major $\mathrm{Fe}$ binding compounds that are present in natural seawater. Extremely low Fe concentrations combined with a highly diverse organic matrix make the chemical characterization of these compounds a formidable analytical challenge.

To structurally characterize ligands in seawater, a preconcentration step is required to remove salt and concentrate the organic ligands. Preconcentration typically involves extraction onto a 
solid resin phase, and many methods have been tested. Acidification to $\mathrm{pH} 2$ yields the highest dissolved organic matter (DOM) extraction efficiencies by protonating functional groups with a low pKa (e.g. carboxylate) to form neutral species with higher affinity for the hydrophobic resin [Dittmar et al., 2008]. However, this method may hydrolyze labile bonds in many siderophores. Thus most studies have focused on the fraction of organic compounds that can be isolated by extraction at seawater $\mathrm{pH}$. This approach works well for a range of known siderophores that have been isolated from marine bacterial cultures, including amphiphilic compounds and ferrioxamines. However, it likely fails to capture some siderophores that may be very polar or deprotonated and negatively charged at seawater $\mathrm{pH}$, such as citrate and vibrioferrin [Loomis and Raymond, 1991; Gledhill and Buck, 2012].

Once extracted, the chemical composition of Fe binding compounds has been investigated by coupling chromatographic separation of organic compounds with detection by mass spectrometry. Two complementary mass spectrometry methods have been used. Inductively coupled plasma mass spectrometry (ICPMS) provides a means to screen samples for the presence of organic-metal complexes. This approach has the major advantage that sensitivity is not dependent on the chemical species of the organic ligand and provides a quantitative measurement of each analyte [Lobiński et al., 2006]. However, it does not provide information on the species identity. Electrospray ionization mass spectrometry (ESIMS) has proven to be a useful complementary technique for determining the mass and fragmentation pattern of the metal-containing compounds and determining their chemical structure [Szpunar, 2005; Mounicou et al., 2009].

The seminal work by Gledhill and colleagues laid the foundation for mass spectrometry based approaches by taking advantage of the similar ligand binding affinities of $\mathrm{Fe}$ and gallium (Ga) [McCormack et al., 2003; Gledhill et al., 2004; Mawji et al., 2008, 2011]. In these studies, they spiked organic extracts with excess gallium, acidified to $\mathrm{pH}<3$ to allow the Ga to swap into siderophores, and analyzed the sample by liquid chromatography mass spectrometry, first using LC-ICPMS to detect the Ga associated with the compounds. The resulting chromatograms yielded information on the number of organic metal ligands in a sample as well as their retention times and abundances (once calibrated with a standard curve). By coupling the same 
chromatography with LC-ESIMS, they were able to identify masses that exhibited the distinctive Ga isotope pattern $\left({ }^{69} \mathrm{Ga}:{ }^{71} \mathrm{Ga}\right.$ of $\left.3: 2\right)$. Further validation of these masses was accomplished by looking for the apo or Fe forms of the compounds in samples that were not spiked with Ga. Early studies used this to investigate seawater glucose enrichments. The added carbon source fueled heterotrophic bacterial growth in these experiments and drove the system into $\mathrm{Fe}$ limited conditions [Gledhill et al., 2004; Mawji et al., 2011]. By generating target compounds in this manner, it was possible to identify several of the ligands in natural seawater as the trishydroxamates ferrioxamine $\mathrm{E}$ and $\mathrm{G}$ [Mawji et al., 2008]. Further studies using carbon enrichments of natural seawater to stimulate heterotrophic production also detected amphibactins along with other compounds that were not identified.

Another promising strategy for identifying siderophores in seawater is by detecting the distinct isotopic ratio of ${ }^{54} \mathrm{Fe}:{ }^{56} \mathrm{Fe}(0.056)$ with high resolution mass spectrometry. When $\mathrm{Fe}$ is incorporated into an organic compound, two isotopologues that reflect the mass difference and relative intensity of the major Fe isotopes are detected. This approach has been used successfully in culture to identify novel siderophores produced by the filamentous fungus Trichoderma and from the nitrogen fixing bacteria Azotobacter vinelandii [Lehner et al., 2013; Baars et al., 2014, 2015]. Isotope-assisted screening has also been used to detect putative novel Fe binding siderophores directly from seawater extracts [Velasquez et al., 2011]. To validate masses of compounds detected by LC-ESIMS and avoid false assignments, some studies have coupled these analyses with rare isotopic labels of Fe and molybdenum (Mo) [Deicke et al., 2014]. Despite these advancements, only two compounds have been identified in natural seawater to date. Improved techniques with lower detection limits combined with computational data analysis approaches are needed to sift through the millions of compounds present in each liter of seawater and identify the fraction that can bind to iron.

This thesis develops these mass spectrometry based approaches into a robust platform for the discovery of Fe binding ligands in seawater. The second chapter describes a trace metal clean LC-ICPMS method for the direct characterization of organic ligands in seawater. This technique overcomes issues with high background and isobaric interferences that have previously hindered sensitive detection of Fe with LC-ICPMS. Detecting Fe directly opens new avenues to 
investigate labile ligands that hydrolyze upon acidification with the Ga method, and enables the quantification of 'Fe bound' versus 'free' ligand in the sample. The third chapter introduces a method for characterizing compounds detected by LC-ICPMS using ESIMS in combination with computer algorithms that align the spectra and search for Fe isotopic features in the ESIMS scans that match the retention time of Fe features in the LC-ICPMS data. Thanks to the superior detection/characterization limits of combined LC-ICPMS/ESIMS, these methods were capable of characterizing new members of the synechobactin suite. Chapters 4 and 5 discuss the application of this method to seawater, revealing distinct ligand compositions across nutrient regimes with ferrioxamines appearing in coastal and oligotrophic surface waters, amphibactins and novel siderophores linked to iron deficient high nutrient low chlorophyll waters, and synechobactins appearing in resuspended sediment layers near the benthic boundary. Finally, Chapter 6 describes a novel method for measuring $\mathrm{Fe}$ exchange rates of individual compounds in a complex mixture, such as seawater, using isotopic exchange monitored by LC-ICPMS. This work highlights for the first time the widespread diversity of organic metal ligands across the ocean and provides fundamental insight into the specific molecular processes that impact iron cycling.

\subsection{References:}

Abualhaija, M. M., H. Whitby, and C. M. G. van den Berg (2015), Competition between copper and iron for humic ligands in estuarine waters, Mar. Chem., 172, 46-56, doi:10.1016/j.marchem.2015.03.010.

De Baar, H., P. Boyd, K. Coale, and M. Landry (2005), Synthesis of iron fertilization experiments: From the Iron Age in the Age of Enlightenment, J. Geophys. Res, 110(C9), 124, doi:10.1029/2004JC002601.

Baars, O., F. M. M. Morel, and D. H. Perlman (2014), ChelomEx: Isotope-Assisted Discovery of Metal Chelates in Complex Media Using High-Resolution LC-MS, Anal. Chem., 86(22), 11298-11305, doi:10.1021/ac503000e.

Baars, O., X. Zhang, F. M. M. Morel, and M. R. Seyedsayamdost (2015), The Siderophore Metabolome of Azotobacter vinelandii, Appl. Environ. Microbiol., (October), AEM.0316015, doi:10.1128/AEM.03160-15.

Barbeau, K. (2006), Photochemistry of Organic Iron(III) Complexing Ligands in Oceanic Systems, Photochem. Photobiol., (82), 1505-1516, doi:10.1562/2006-06-16-1R-935. 
Barbeau, K., E. L. Rue, C. G. Trick, K. W. Bruland, and A. Butler (2003), Photochemical reactivity of siderophores produced by marine heterotrophic bacteria and cyanobacteria, based on characteristic Fe(III) binding groups, Limnol. Oceanogr., 48(3), 1069-1078, doi:10.4319/1o.2003.48.3.1069.

Barbeau, K. A., E. L. Rue, K. W. Bruland, and A. Butler (2001), Photochemical cycling of iron in the surface ocean mediated by microbial iron(III)-binding ligands., Nature, 413(6854), 409-13, doi:10.1038/35096545.

Boyd, P. W., and M. J. Ellwood (2010), The biogeochemical cycle of iron in the ocean, Nat. Geosci., 3(10), 675-682, doi:10.1038/ngeo964.

Boyd, P. W., and A. Tagliabue (2015), Using the L* concept to explore controls on the relationship between paired ligand and dissolved iron concentrations in the ocean, Mar. Chem., 173, 52-66, doi:10.1016/j.marchem.2014.12.003.

Boyd, P. W. et al. (2007), Mesoscale Iron Enrichment Experiments 1993-2005: Synthesis and Future Directions, Science, 315(February), 612-617.

Boye, M., J. Nishioka, P. Croot, P. Laan, K. R. Timmermans, V. H. Strass, S. Takeda, and H. J. W. de Baar (2010), Significant portion of dissolved organic Fe complexes in fact is Fe colloids, Mar. Chem., 122(1-4), 20-27, doi:10.1016/j.marchem.2010.09.001.

Boyle, E. A., B. A. Bergquist, R. A. Kayser, and N. Mahowald (2005), Iron, manganese, and lead at Hawaii Ocean Time-series station ALOHA: Temporal variability and an intermediate water hydrothermal plume, Geochim. Cosmochim. Acta, 69(4), 933-952, doi:10.1016/j.gca.2004.07.034.

Braun, V., and K. Hantke (2013), Iron Uptake in Bacteria with Emphasis on E. coli and Pseudomonas, SpringerBriefs in Molecular Science, edited by R. Chakraborty, V. Braun, K. Hantke, and P. Cornelis, Springer Netherlands, Dordrecht.

Bruland, K. W., J. R. Donat, and D. A. Hutchins (1991), Interactive influences of bioactive trace metals on biological production in oceanic waters, Limnol. Oceanogr., 36(8), 1555-1577, doi:10.4319/lo.1991.36.8.1555.

Bruland, K. W., R. Middag, and M. C. Lohan (2013), Controls of Trace Metals in Seawater, 2nd ed., Elsevier Ltd.

Buck, K., and K. Bruland (2007), The physicochemical speciation of dissolved iron in the Bering Sea , Alaska, Limnol. Oceanogr., 52(5), 1800-1808.

Buck, K., M. Lohan, C. Berger, and K. Bruland (2007), Dissolved iron speciation in two distinct river plumes and an estuary : Implications for riverine iron supply, Limnol. Oceanogr., 52(2), 843-855. 
Bundy, R. M., H. A. N. Abdulla, P. G. Hatcher, D. V. Biller, K. N. Buck, and K. a. Barbeau (2015a), Iron-binding ligands and humic substances in the San Francisco Bay estuary and estuarine-influenced shelf regions of coastal California, Mar. Chem., 173, 183-194, doi:10.1016/j.marchem.2014.11.005.

Bundy, R. M., H. A. N. Abdulla, P. G. Hatcher, D. V. Biller, K. N. Buck, and K. A. Barbeau (2015b), Iron-binding ligands and humic substances in the San Francisco Bay estuary and estuarine-influenced shelf regions of coastal California, Mar. Chem., 173, 183-194, doi:10.1016/j.marchem.2014.11.005.

Butler, A. (1998), Acquisition and Utilization of Transition Metal Ions by Marine Organisms, Science, 281(5374), 207-209, doi:10.1126/science.281.5374.207.

Conway, T. M., and S. G. John (2014), Quantification of dissolved iron sources to the North Atlantic Ocean, Nature, 511(7508), 212-215, doi:10.1038/nature13482.

Cordero, O. X., L. A. Ventouras, E. F. DeLong, and M. F. Polz (2012), Public good dynamics drive evolution of iron acquisition strategies in natural bacterioplankton populations, Proc. Natl. Acad. Sci., 1-6, doi:10.1073/pnas.1213344109.

Cullen, J. T., B. A. Bergquist, and J. W. Moffett (2006), Thermodynamic characterization of the partitioning of iron between soluble and colloidal species in the Atlantic Ocean, Mar. Chem., 98(2-4), 295-303, doi:10.1016/j.marchem.2005.10.007.

Deicke, M., J. F. Mohr, J.-P. Bellenger, and T. Wichard (2014), Metallophore mapping in complex matrices by metal isotope coded profiling of organic ligands, Analyst, 139, 60966099, doi:10.1039/C4AN01461H.

Dittmar, T., B. Koch, N. Hertkorn, and G. Kattner (2008), A simple and efficient method for the solid-phase extraction of dissolved organic matter (SPE-DOM) from seawater, Limnol. Ocean. Methods, 6, 230-235.

Dutkiewicz, S., B. A. Ward, F. Monteiro, and M. J. Follows (2012), Interconnection of nitrogen fixers and iron in the Pacific Ocean: Theory and numerical simulations, Global Biogeochem. Cycles, 26(1), n/a-n/a, doi:10.1029/2011GB004039.

Fitzsimmons, J. N., E. A. Boyle, and W. J. Jenkins (2014a), Distal transport of dissolved hydrothermal iron in the deep South Pacific Ocean, Proc. Natl. Acad. Sci., 1-8, doi:10.1073/pnas.1418778111.

Fitzsimmons, J. N., R. M. Bundy, S. N. Al-Subiai, K. A. Barbeau, and E. A. Boyle (2014b), The composition of dissolved iron in the dusty surface ocean: An exploration using sizefractionated iron-binding ligands, Mar. Chem., doi:10.1016/j.marchem.2014.09.002.

Fitzsimmons, J. N., C. T. Hayes, S. Al-Subiai, R. Zhang, P. Morton, R. Weisend, F. Ascani, and 
E. A. Boyle (2015a), Daily to Decadal variability of size-fractionated iron and iron-binding ligands at the Hawaii Ocean Time-series Station ALOHA Article, Geochim. Cosmochim. Acta, SUBMITTED, 303-324, doi:10.1016/j.gca.2015.08.012.

Fitzsimmons, J. N., G. G. Carrasco, J. Wu, S. Roshan, M. Hatta, C. I. Measures, T. M. Conway, S. G. John, and E. A. Boyle (2015b), Deep-Sea Research II Partitioning of dissolved iron and iron isotopes into soluble and colloidal phases along the GA03 GEOTRACES North Atlantic Transect, Deep. Res. Part II, 1-22, doi:10.1016/j.dsr2.2014.11.014.

Follows, M. J., S. Dutkiewicz, S. Grant, and S. W. Chisholm (2007), Emergent biogeography of microbial communities in a model ocean., Science, 315(5820), 1843-6, doi:10.1126/science.1138544.

Gauglitz, J. M., and A. Butler (2013), Amino acid variability in the peptide composition of a suite of amphiphilic peptide siderophores from an open ocean Vibrio species., J. Biol. Inorg. Chem., 18(5), 489-97, doi:10.1007/s00775-013-0995-3.

Gledhill, M. (2007), The determination of heme b in marine phyto- and bacterioplankton, Mar. Chem., 103(3-4), 393-403, doi:10.1016/j.marchem.2006.10.008.

Gledhill, M. (2014), The detection of iron protoporphyrin (heme b) in phytoplankton and marine particulate material by electrospray ionisation mass spectrometry - comparison with diode array detection., Anal. Chim. Acta, 841, 33-43, doi:10.1016/j.aca.2014.06.045.

Gledhill, M., and C. M. G. van den Berg (1994), Determination of complexation of iron (III) with natural organic complexing ligands in seawater using cathodic stripping voltammetry, Mar. Chem., 47(1), 41-54, doi:10.1016/0304-4203(94)90012-4.

Gledhill, M., and C. M. G. van den Berg (1995), Measurement of the redox speciation of iron in seawater by catalytic cathodic stripping voltammetry, Mar. Chem., 50(1-4), 51-61, doi:10.1016/0304-4203(95)00026-N.

Gledhill, M., and K. N. Buck (2012), The organic complexation of iron in the marine environment: a review., Front. Microbiol., 3, 1-17, doi:10.3389/fmicb.2012.00069.

Gledhill, M., P. McCormack, S. Ussher, E. P. Achterberg, R. F. C. Mantoura, and P. J. Worsfold (2004), Production of siderophore type chelates by mixed bacterioplankton populations in nutrient enriched seawater incubations, Mar. Chem., 88(1-2), 75-83, doi:10.1016/j.marchem.2004.03.003.

Hansard, S. P., W. M. Landing, C. I. Measures, and B. M. Voelker (2009), Dissolved iron(II) in the Pacific Ocean: Measurements from the PO2 and P16N CLIVAR/CO2 repeat hydrography expeditions, Deep Sea Res. Part I Oceanogr. Res. Pap., 56(7), 1117-1129, doi:10.1016/j.dsr.2009.03.006. 
Hassler, C. S., E. Alasonati, C. A. Mancuso Nichols, and V. I. Slaveykova (2011a), Exopolysaccharides produced by bacteria isolated from the pelagic Southern Ocean - Role in Fe binding, chemical reactivity, and bioavailability, Mar. Chem., 123(1-4), 88-98, doi:10.1016/j.marchem.2010.10.003.

Hassler, C. S., V. Schoemann, C. M. Nichols, E. C. V. Butler, and P. W. Boyd (2011b), Saccharides enhance iron bioavailability to Southern Ocean phytoplankton, Proc. Natl. Acad. Sci., 108(3), 1076-1081, doi:10.1073/pnas.1010963108.

Hassler, C. S., L. Norman, C. A. Mancuso Nichols, L. A. Clementson, C. Robinson, V. Schoemann, R. J. Watson, and M. A. Doblin (2014), Iron associated with exopolymeric substances is highly bioavailable to oceanic phytoplankton, Mar. Chem., doi:10.1016/j.marchem.2014.10.002.

Hawkes, J. A., D. P. Connelly, M. Gledhill, and E. P. Achterberg (2013), The stabilisation and transportation of dissolved iron from high temperature hydrothermal vent systems, Earth Planet. Sci. Lett., 375, 280-290, doi:10.1016/j.eps1.2013.05.047.

Hayes, C. T., J. N. Fitzsimmons, E. A. Boyle, D. McGee, R. F. Anderson, R. Weisend, and P. L. Morton (2015), Thorium isotopes tracing the iron cycle at the Hawaii Ocean Time-series Station ALOHA, Geochim. Cosmochim. Acta, 169, 1-16, doi:10.1016/j.gca.2015.07.019.

Hertkorn, N., R. Benner, M. Frommberger, P. Schmittkopplin, M. Witt, K. Kaiser, A. Kettrup, and J. Hedges (2006), Characterization of a major refractory component of marine dissolved organic matter, Geochim. Cosmochim. Acta, 70(12), 2990-3010, doi:10.1016/j.gca.2006.03.021.

Hertkorn, N., C. Ruecker, M. Meringer, R. Gugisch, M. Frommberger, E. M. Perdue, M. Witt, and P. Schmitt-Kopplin (2007), High-precision frequency measurements: indispensable tools at the core of the molecular-level analysis of complex systems., Anal. Bioanal. Chem., 389(5), 1311-27, doi:10.1007/s00216-007-1577-4.

Ho, T.-Y., A. Quigg, Z. V. Finkel, A. J. Milligan, K. Wyman, P. G. Falkowski, and F. M. M. Morel (2003), The elemental composition of some marine phytoplankton, J. Phycol., 39(6), 1145-1159, doi:10.1111/j.0022-3646.2003.03-090.x.

Hogle, S. L., K. A. Barbeau, and M. Gledhill (2014), Heme in the marine environment: from cells to the iron cycle., Metallomics, 6(6), 1107-20, doi:10.1039/c4mt00031e.

Holt, P. D., R. R. Reid, B. L. Lewis, G. W. Luther, and A. Butler (2005), Iron(III) coordination chemistry of alterobactin A: a siderophore from the marine bacterium Alteromonas luteoviolacea., Inorg. Chem., 44(21), 7671-7, doi:10.1021/ic0512072.

Homann, V. V, M. Sandy, J. A. Tincu, A. S. Templeton, B. M. Tebo, and A. Butler (2009a), Loihichelins A-F, a suite of amphiphilic siderophores produced by the marine bacterium 
Halomonas LOB-5., J. Nat. Prod., 72(5), 884-8, doi:10.1021/np800640h.

Homann, V. V, K. J. Edwards, E. a Webb, and A. Butler (2009b), Siderophores of Marinobacter aquaeolei: petrobactin and its sulfonated derivatives., Biometals, 22(4), 565-71, doi:10.1007/s10534-009-9237-0.

Hopkinson, B. M., and K. A. Barbeau (2007), Organic and redox speciation of iron in the eastern tropical North Pacific suboxic zone, Mar. Chem., 106(1-2), 2-17, doi:10.1016/j.marchem.2006.02.008.

Hopkinson, B. M., and K. A. Barbeau (2012), Iron transporters in marine prokaryotic genomes and metagenomes., Environ. Microbiol., 14(1), 114-28, doi:10.1111/j.14622920.2011.02539.x.

Hopkinson, B. M., and F. M. M. Morel (2009), The role of siderophores in iron acquisition by photosynthetic marine microorganisms., Biometals, 22(4), 659-69, doi:10.1007/s10534009-9235-2.

Hutchins, D. A., A. E. Witter, A. Butler, and G. W. Luther (1999), Competition among marine phytoplankton for different chelated iron species, Nature, 400(6747), 858-861.

Ito, Y., and A. Butler (2005), Structure of synechobactins, new siderophores of the marine cyanobacterium Synechococcus sp. PCC 7002, Limnol. Oceanogr., 50(6), 1918-1923, doi:10.4319/1o.2005.50.6.1918.

Jickells, T. D. et al. (2005), Global iron connections between desert dust, ocean biogeochemistry, and climate, Science, 308(67), 67-71, doi:10.1126/science.1105959.

Kanoh, K., K. Kamino, G. Leleo, K. Adachi, and Y. Shizuri (2003), Pseudoalterobactin A and B, New Siderophores Excreted by Marine Bacterium Pseudoalteromonas sp. KP20-4., J. Antibiot. (Tokyo)., 56(10), 871-875.

Kieber, R. J., S. a Skrabal, B. J. Smith, and J. D. Willey (2005), Organic complexation of Fe(II) and its impact on the redox cycling of iron in rain., Environ. Sci. Technol., 39(6), 1576-83.

Kranzler, C., H. Lis, Y. Shaked, and N. Keren (2011), The role of reduction in iron uptake processes in a unicellular, planktonic cyanobacterium., Environ. Microbiol., 13(11), 2990 9, doi:10.1111/j.1462-2920.2011.02572.x.

Kustka, A. B., Y. Shaked, A. J. Milligan, D. W. King, and F. M. M. Morel (2005), Extracellular production of superoxide by marine diatoms: Contrasting effects on iron redox chemistry and bioavailability, Limnol. Oceanogr., 50(4), 1172-1180, doi:10.4319/lo.2005.50.4.1172.

Laglera, L. M., and C. M. G. van den Berg (2009), Evidence for geochemical control of iron by humic substances in seawater, Limnol. Oceanogr., 54(2), 610-619. 
Laglera, L. M., G. Battaglia, and C. M. G. van den Berg (2007), Determination of humic substances in natural waters by cathodic stripping voltammetry of their complexes with iron., Anal. Chim. Acta, 599(1), 58-66, doi:10.1016/j.aca.2007.07.059.

Laglera, L. M., G. Battaglia, and C. M. G. van den Berg (2011), Effect of humic substances on the iron speciation in natural waters by CLE/CSV, Mar. Chem., 127(1-4), 134-143, doi:10.1016/j.marchem.2011.09.003.

Leão, P. N., M. T. S. D. Vasconcelos, and V. M. Vasconcelos (2007), Role of marine cyanobacteria in trace metal bioavailability in seawater., Microb. Ecol., 53(1), 104-9, doi:10.1007/s00248-006-9153-6.

Lehner, S. M., L. Atanasova, N. K. N. Neumann, R. Krska, M. Lemmens, I. S. Druzhinina, and R. Schuhmacher (2013), Isotope-assisted screening for iron-containing metabolites reveals a high degree of diversity among known and unknown siderophores produced by Trichoderma spp., Appl. Environ. Microbiol., 79(1), 18-31, doi:10.1128/AEM.02339-12.

Liu, X., and F. Millero (2002), The solubility of iron in seawater, Mar. Chem., 77, 43-54.

Lobiński, R., D. Schaumlöffel, and J. Szpunar (2006), Mass spectrometry in bioinorganic analytical chemistry., Mass Spectrom. Rev., 25(2), 255-89, doi:10.1002/mas.20069.

Lohan, M. C., and K. W. Bruland (2008), Elevated Fe(II) and dissolved Fe in hypoxic shelf waters off Oregon and Washington: An enhanced source of iron to coastal upwelling regimes, Environ. Sci. Technol., 42(17), 6462-6468, doi:10.1021/es800144j.

Loomis, L. D., and K. N. Raymond (1991), Solution Equilibria of Enterobactin and MetalEnterobactin Complexes, Inorg. Chem., 30(1), 906-911, doi:10.1021/ic00005a008.

Maldonado, M. T., and N. M. Price (2001), Reduction and Transport of Organically Bound Iron By Thalassiosira Oceanica (Bacillariophyceae), J. Phycol., 37(2), 298-310, doi:10.1046/j.1529-8817.2001.037002298.x.

Malmstrom, R. R., S. Rodrigue, K. H. Huang, L. Kelly, S. E. Kern, A. Thompson, S. Roggensack, P. M. Berube, M. R. Henn, and S. W. Chisholm (2013), Ecology of uncultured Prochlorococcus clades revealed through single-cell genomics and biogeographic analysis., ISME J., 7, 184-198, doi:10.1038/ismej.2012.89.

Maranger, R., D. F. Bird, and N. M. Price (1998), Iron acquisition by photosynthetic marine phytoplankton from ingested bacteria, Nature, 396(November), 248-251.

Martin, J. D., Y. Ito, V. V Homann, M. G. Haygood, and A. Butler (2006), Structure and membrane affinity of new amphiphilic siderophores produced by Ochrobactrum sp. SP18., J. Biol. Inorg. Chem., 11(5), 633-41, doi:10.1007/s00775-006-0112-y.

Martin, J. H., and S. E. Fitzwater (1988), Iron deficiency limits phytoplankton growth in the 
north-east Pacific subarctic, Nature, 331(28), 341-343.

Martin, J. H., S. E. Fitzwater, and R. M. Gordon (1990), Iron deficiency limits phytoplankton growth in antarctic waters, Global Biogeochem. Cycles, 4(Figure 1).

Martin, J. H., R. M. Gordon, and S. E. Fitzwater (1991), The case for iron, Limnol. Oceanogr., 36(8), 1793-1802.

Martin, J. H. et al. (1994), Testing the iron hypothesis in ecosystems of the equatorial Pacific Ocean, Nature, 371(6493), 123-129.

Martinez, J. S., and A. Butler (2007), Marine amphiphilic siderophores: marinobactin structure, uptake, and microbial partitioning, J. Inorg. Biochem., 101(805), 1692-1698, doi:10.1016/j.jinorgbio.2007.07.007.Marine.

Martinez, J. S., M. G. Haygood, and A. Butler (2001), Identification of a natural desferrioxamine siderophore produced by a marine bacterium, Limnol. Oceanogr., 46(2), 420-424, doi:10.4319/1o.2001.46.2.0420.

Martinez, J. S., J. N. Carter-Franklin, E. L. Mann, J. D. Martin, M. G. Haygood, and A. Butler (2003), Structure and membrane affinity of a suite of amphiphilic siderophores produced by a marine bacterium., Proc. Natl. Acad. Sci. U. S. A., 100(7), 3754-9, doi:10.1073/pnas.0637444100.

Mawji, E., M. Gledhill, J. A. Milton, G. A. Tarran, S. Ussher, A. Thompson, G. A. Wolff, P. J. Worsfold, and E. P. Achterberg (2008), Hydroxamate siderophores: occurrence and importance in the Atlantic Ocean., Environ. Sci. Technol., 42(23), 8675-80.

Mawji, E., M. Gledhill, J. A. Milton, M. V. Zubkov, A. Thompson, G. A. Wolff, and E. P. Achterberg (2011), Production of siderophore type chelates in Atlantic Ocean waters enriched with different carbon and nitrogen sources, Mar. Chem., 124(1-4), 90-99, doi:10.1016/j.marchem.2010.12.005.

McCormack, P., P. J. Worsfold, and M. Gledhill (2003), Separation and detection of siderophores produced by marine bacterioplankton using high-performance liquid chromatography with electrospray ionization mass spectrometry., Anal. Chem., 75(11), 2647-52.

Miller, W. L., and R. G. Zepp (1995), Photochemical production of dissolved inorganic carbon from terrestrial organic matter: Significance to the oceanic organic carbon cycle, Geophys. Res. Lett., 22(4), 417-420.

Misumi, K., K. Lindsay, J. K. Moore, S. C. Doney, D. Tsumune, and Y. Yoshida (2013), Humic substances may control dissolved iron distributions in the global ocean: Implications from numerical simulations, Global Biogeochem. Cycles, 27(2), 450-462, 
doi:10.1002/gbc.20039.

Moore, J. K., and O. Braucher (2008), Sedimentary and mineral dust sources of dissolved iron to the World Ocean, Biogeosciences, 5, 631-656, doi:10.5194/bgd-4-1279-2007.

Moore, J. K., and S. C. Doney (2007), Iron availability limits the ocean nitrogen inventory stabilizing feedbacks between marine denitrification and nitrogen fixation, Global Biogeochem. Cycles, 21(2), doi:10.1029/2006GB002762.

Moore, J. K., S. C. Doney, D. M. Glover, and I. Y. Fung (2002), Iron cycling and nutrientlimitation patterns in surface waters of the World Ocean, Deep Sea Res. II, 49, 463-507.

Moore, J. K., S. C. Doney, and K. Lindsay (2004), Upper ocean ecosystem dynamics and iron cycling in a global three-dimensional model, Global Biogeochem. Cycles, 18(4), 1-21, doi:10.1029/2004GB002220.

Morel, F. M. M., and N. M. Price (2003), The biogeochemical cycles of trace metals in the oceans., Science, 300(5621), 944-7, doi:10.1126/science.1083545.

Morel, F. M. M., A. J. Milligan, and M. A. Saito (2003), Marine Bioinorganic Chemistry : The Role of Trace Metals in the Oceanic Cycles of Major Nutrients, Treatise Geochemistry, Vol. 6.

Morel, F. M. M., A. B. Kustka, and Y. Shaked (2008), The role of unchelated Fe in the iron nutrition of phytoplankton, Limnol. Oceanogr., 53(1), 400-404.

Mounicou, S., J. Szpunar, and R. Lobinski (2009), Metallomics: the concept and methodology., Chem. Soc. Rev., 38(4), 1119-38, doi:10.1039/b713633c.

Norman, L., I. A. M. Worms, E. Angles, A. R. Bowie, C. M. Nichols, A. Ninh Pham, V. I. Slaveykova, A. T. Townsend, T. David Waite, and C. S. Hassler (2015), The role of bacterial and algal exopolymeric substances in iron chemistry, Mar. Chem., 173, 148-161, doi:10.1016/j.marchem.2015.03.015.

Ratledge, C., and L. G. Dover (2000), Iron Metabolism in Pathogenic Bacteria, Annu. Rev. Microbiol., 54, 881-941.

Repeta, D. J. (2015), Chemical characterization and cycling of dissolved organic matter, in Biogeochemistry of Marine Dissolved Organic Matter, edited by D. A. Hansell and C. A. Carlson, Elsevier.

Resing, J. A., P. N. Sedwick, C. R. German, W. J. Jenkins, J. W. Moffett, B. M. Sohst, and A. Tagliabue (2015), Basin-scale transport of hydrothermal dissolved metals across the South Pacific Ocean, Nature, 523(7559), 200-203, doi:10.1038/nature14577.

Roe, K. L., K. Barbeau, E. L. Mann, and M. G. Haygood (2012), Acquisition of iron by 
Trichodesmium and associated bacteria in culture., Environ. Microbiol., 14(7), 1681-95, doi:10.1111/j.1462-2920.2011.02653.x.

Roe, K. L., S. L. Hogle, and K. A. Barbeau (2013), Utilization of heme as an iron source by marine Alphaproteobacteria in the Roseobacter clade., Appl. Environ. Microbiol., (July), 139, doi:10.1128/AEM.01562-13.

Roy, E. G., and M. L. Wells (2011), Evidence for regulation of Fe(II) oxidation by organic complexing ligands in the Eastern Subarctic Pacific, Mar. Chem., 127(1-4), 115-122, doi:10.1016/j.marchem.2011.08.006.

Roy, E. G., M. L. Wells, and D. W. King (2008), Persistence of iron(II) in surface waters of the western subarctic Pacific, Limnol. Oceanogr., 53(1), 89-98, doi:10.4319/lo.2008.53.1.0089.

Rue, E., and K. Bruland (1995), Complexation of iron(III) by natural organic ligands in the Central North Pacific as determined by a new competitive ligand equilibration/adsorptive cathodic stripping voltammetric method, Mar. Chem., 50(1-4), 117-138, doi:10.1016/03044203(95)00031-L.

Rue, E. L., and K. W. Bruland (1997), The role of organic complexation on ambient iron chemistry in the equatorial Pacific Ocean and the response of a mesoscale iron addition experiment, Limnol. Oceanogr., 42(5), 901-910, doi:10.4319/1o.1997.42.5.0901.

Saito, M. A., A. E. Noble, A. Tagliabue, T. J. Goepfert, C. H. Lamborg, and W. J. Jenkins (2013), Slow-spreading submarine ridges in the South Atlantic as a significant oceanic iron source, Nat. Geosci., 6(9), 775-779, doi:10.1038/ngeo1893.

Salmon, T. P., A. L. Rose, B. A. Neilan, and T. D. Waite (2006), The FeL model of iron acquisition: Nondissociative reduction of ferric complexes in the marine environment, Limnol. Oceanogr., 51(4), 1744-1754, doi:10.4319/lo.2006.51.4.1744.

Sandy, M., and A. Butler (2009), Microbial iron acquisition: marine and terrestrial siderophores., Chem. Rev., 109(10), 4580-95, doi:10.1021/cr9002787.

Sandy, M., A. Han, J. Blunt, M. Munro, M. Haygood, and A. Butler (2010), Vanchrobactin and anguibactin siderophores produced by Vibrio sp. DS40M4., J. Nat. Prod., 73(6), 1038-43, doi:10.1021/np900750g.

Schlosser, C., and P. L. Croot (2009), Controls on seawater Fe(III) solubility in the Mauritanian upwelling zone, Geophys. Res. Lett., 36(18), L18606, doi:10.1029/2009GL038963.

Shaked, Y., A. B. Kustka, and F. M. M. Morel (2005), A general kinetic model for iron acquisition by eukaryotic phytoplankton, Limnol. Oceanogr., 50(3), 872-882.

Szpunar, J. (2005), Advances in analytical methodology for bioinorganic speciation analysis: metallomics, metalloproteomics and heteroatom-tagged proteomics and metabolomics., 
Analyst, 130(4), 442-65, doi:10.1039/b418265k.

Tagliabue, A., and C. Völker (2011), Towards accounting for dissolved iron speciation in global ocean models, Biogeosciences, 8(10), 3025-3039, doi:10.5194/bg-8-3025-2011.

Tagliabue, A., T. Mtshali, O. Aumont, A. R. Bowie, M. B. Klunder, A. N. Roychoudhury, and S. Swart (2012), A global compilation of dissolved iron measurements: Focus on distributions and processes in the Southern Ocean, Biogeosciences, 9, 2333-2349, doi:10.5194/bg-92333-2012.

Telford, J. R., and K. N. Raymond (1997), Amonabactin: a family of novel siderophores from a pathogenic bacterium, J. Biol. Inorg. Chem., 2(6), 750-761, doi:10.1007/s007750050191.

Thuróczy, C.-E., L. J. a. Gerringa, M. B. Klunder, R. Middag, P. Laan, K. R. Timmermans, and H. J. W. de Baar (2010), Speciation of Fe in the Eastern North Atlantic Ocean, Deep Sea Res. Part I Oceanogr. Res. Pap., 57(11), 1444-1453, doi:10.1016/j.dsr.2010.08.004.

Toulza, E., A. Tagliabue, S. Blain, and G. Piganeau (2012), Analysis of the global ocean sampling (GOS) project for trends in iron uptake by surface ocean microbes., PLoS One, 7(2), e30931, doi:10.1371/journal.pone.0030931.

Turner, D., M. Whitfield, and A. Dickson (1981), The equilibrium speciation of dissolved components in freshwater and sea water at $25^{\circ} \mathrm{C}$ and $1 \mathrm{~atm}$ pressure, Geochim. Cosmochim. Acta, 45(6), 855-881, doi:10.1016/0016-7037(81)90115-0.

Twining, B. S., and S. B. Baines (2011), The Trace Metal Composition of Marine Phytoplankton, Ann. Rev. Mar. Sci., 5(1), 120830114950002, doi:10.1146/annurev-marine121211-172322.

Ussher, S. J., E. P. Achterberg, and P. J. Worsfold (2004), Marine biogeochemistry of iron, Environ. Chem., 1(2), 67-80, doi:10.1071/EN04053.

Velasquez, I., B. L. Nunn, E. Ibisanmi, D. R. Goodlett, K. A. Hunter, and S. G. Sander (2011), Detection of hydroxamate siderophores in coastal and Sub-Antarctic waters off the South Eastern Coast of New Zealand, Mar. Chem., 126(1-4), 97-107, doi:10.1016/j.marchem.2011.04.003.

Völker, C., and A. Tagliabue (2014), Modeling organic iron-binding ligands in a threedimensional biogeochemical ocean model, Mar. Chem., doi:10.1016/j.marchem.2014.11.008.

Vraspir, J. M., P. D. Holt, and A. Butler (2011), Identification of new members within suites of amphiphilic marine siderophores., Biometals, 24(1), 85-92, doi:10.1007/s10534-010-93781.

Wandersman, C., and P. Delepelaire (2004), Bacterial iron sources: from siderophores to 
hemophores., Annu. Rev. Microbiol., 58, 611-47, doi:10.1146/annurev.micro.58.030603.123811.

Wells, M. L., and E. D. Goldberg (1992), Marine submicron particles, Mar. Chem., 40(1-2), 518, doi:10.1016/0304-4203(92)90045-C.

Wells, M. L., E. D. Goldberg, and L. Jolla (1994), The distribution of colloids in the North Atlantic Southern Oceans, Limnol. Oceanogr., 39(2), 286-302, doi:10.4319/lo.1994.39.2.0286.

Willey, J. D., R. J. Kieber, P. J. Seaton, and C. Miller (2008), Rainwater as a source of Fe(II)stabilizing ligands to seawater, Limnol. Oceanogr., 53(4), 1678-1684.

Worsfold, P. J., M. C. Lohan, S. J. Ussher, and A. R. Bowie (2014), Determination of dissolved iron in seawater : A historical review, Mar. Chem., 166, 25-35, doi:10.1016/j.marchem.2014.08.009.

Wu, J., and G. Luther (1995), Complexation of Fe(III) by natural organic ligands in the Northwest Atlantic Ocean by a competitive ligand equilibration method and a kinetic approach, Mar. Chem., 50(1-4), 159-177, doi:10.1016/0304-4203(95)00033-N.

Wu, J., E. A. Boyle, W. Sunda, and L. S. Wen (2001), Soluble and colloidal iron in the oligotrophic North Atlantic and North Pacific., Science, 293(5531), 847-9, doi:10.1126/science.1059251.

Xu, G., J. S. Martinez, J. T. Groves, and A. Butler (2002), Membrane affinity of the amphiphilic marinobactin siderophores., J. Am. Chem. Soc., 124(45), 13408-15.

Yücel, M., A. Gartman, C. S. Chan, and G. W. Luther (2011), Hydrothermal vents as a kinetically stable source of iron-sulphide-bearing nanoparticles to the ocean, Nat. Geosci., 4(6), 367-371, doi:10.1038/ngeo1148.

Zhang, G., S. A. Amin, F. C. Küpper, P. D. Holt, C. J. Carrano, and A. Butler (2009), Ferric stability constants of representative marine siderophores: marinobactins, aquachelins, and petrobactin., Inorg. Chem., 48(23), 11466-73, doi:10.1021/ic901739m. 


\title{
Chapter 2: Detection of iron ligands in seawater and marine cyanobacteria cultures by HPLC-ICP-MS
}

Reproduced with permission from "Boiteau, R. M., Fitzsimmons, J. N., Repeta, D. J. \& Boyle, E. A. Detection of iron ligands in seawater and marine cyanobacteria cultures by highperformance liquid chromatography-inductively coupled plasma-mass spectrometry. Anal. Chem. 85, 4357-62 (2013)." Copyright 2013 American Chemical Society.

\begin{abstract}
:
Organic ligands dominate the speciation of iron in the ocean. Little is known, however, about the chemical composition and distribution of these compounds. Here we describe a method to detect low concentrations of organic Fe ligands using reverse phase high-performance liquid chromatography (HPLC) tandem multi-collector inductively coupled plasma mass spectrometry (MC-ICP-MS). This technique can be used to screen seawater and marine cultures for target compounds that can be isolated and structurally characterized. Sensitive detection $(<1$ picomole $\mathrm{Fe}$ ) is achieved by using an iron-free HPLC system to reduce background Fe levels, minimizing ${ }^{40} \mathrm{Ar}^{16} \mathrm{O}^{+}$interferences on ${ }^{56} \mathrm{Fe}$ with a hexapole collision cell, and introducing oxygen into the sample carrier gas to prevent the formation of reduced carbon deposits that decrease sensitivity. This method was tested with a chromatographic separation of five trace metal complexes that represent the polarity range likely found in seawater. Good separation was achieved with a 20 min water/methanol gradient, although sensitivity decreased by a factor of two at high organic solvent concentrations. Finally, Fe ligand complexes were detected from the organic extract of surface South Pacific seawater and from culture media of the siderophore producing cyanobacteria Synechococcus sp. PCC 7002.
\end{abstract}

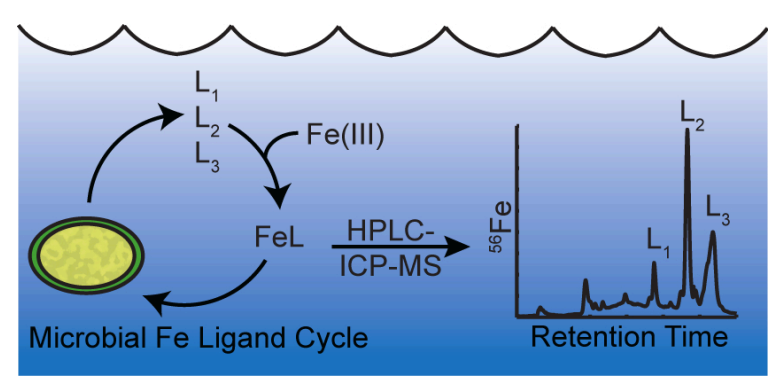




\subsection{Introduction:}

Iron $(\mathrm{Fe})$ is an essential micronutrient for marine microbes,[Bruland et al., 1991; Morel and Price, 2003; Morel et al., 2003; Leão et al., 2007] yet the concentrations of dissolved Fe in surface waters are low $(0.02 \mathrm{nM}$ to $<1 \mathrm{nM})$.[Boyd and Ellwood, 2010] As a result, primary productivity and carbon export are limited by $\mathrm{Fe}$ in regions where the inputs from dust deposition and upwelling are scarce.[De Baar et al., 2005; Jickells et al., 2005; Boyd et al., 2007] The solubility and bioavailability of iron and other trace metals such as cobalt, nickel, copper, zinc and cadmium in the ocean are regulated by organic ligands.[Vraspir and Butler, 2009] Electrochemical measurements of bulk Fe(III) ligand concentration and binding strength suggest that this ligand pool ranges from $1-2 \mathrm{nM}$ and complexes over $99 \%$ of dissolved iron (Fe) in the ocean.[Rue and Bruland, 1995; Wu and Luther, 1995; Boye et al., 2001; Vraspir and Butler, 2009] However, the molecular structures, sources and fates of these ligands are currently unknown.

The biogeochemical importance of organic ligands has sparked interest in developing sensitive, trace metal clean analytical methods that can be coupled with chromatography for the characterization of specific ligands. High pressure liquid chromatography (HPLC) tandem electrospray ionization mass spectrometry (HPLC-ESI-MS) has been used to characterize specific ligands from bulk organic samples. With this method, recent studies have detected putative Fe ligands in seawater by identifying compounds with distinctive metal isotope patterns.[McCormack et al., 2003; Gledhill et al., 2004; Mawji et al., 2008, 2011; Velasquez et al., 2011] MS/MS analysis of these compounds suggest that some are similar to hydroxamate siderophores, which are produced by microbes to facilitate Fe uptake under low Fe conditions.

In this study, we present a complementary method for directly detecting the Fe associated with specific organic compounds by HPLC-tandem inductively coupled plasma mass spectrometry (ICP-MS). HPLC-ICP-MS offers universal detection of metal complexes in an organic extract sample with minimal sample manipulation and can simultaneously detect multiple metals in real time. With this technique, seawater samples can be rapidly screened to determine the number and relative polarities of low concentration metal ligand complexes. By splitting the flow eluting 
from the HPLC column, Fe-ligand complexes can be detected in real time and recovered by fraction collection, allowing structural characterization by ESI-MS and other methods. Although HPLC-ICP-MS has previously been shown to provide rapid and sensitive detection of metalorganic complexes from environmental samples (such as $\mathrm{Ag}, \mathrm{Cd}, \mathrm{Cu}, \mathrm{Mo}, \mathrm{Ni}, \mathrm{Pb}, \mathrm{Tl}, \mathrm{U}, \mathrm{W}, \mathrm{Zn}$ and Zr),[Heumann et al., 1994; Rottmann and Heumann, 1994; Vogl and Heumann, 1997, 1998; Ammann, 2002; Lechtenfeld et al., 2011] monitoring Fe by HPLC-ICP-MS is complicated by high procedural blanks, isobaric argon oxide mass interferences in ICP-MS, and the very low concentrations of Fe in marine samples.[Harrington et al., 2001; Gledhill and Buck, 2012]

The method described here employs several strategies for reducing ${ }^{56} \mathrm{Fe}$ backgrounds and interferences to achieve sensitive, quantitative detection of $\mathrm{Fe}$ ligands. Low $\mathrm{Fe}$ blanks are achieved with the use of a bioinert titanium/peek HPLC system and distilled organic solvents. Secondly, a hexapole collision cell in the ICP-MS minimizes ${ }^{40} \mathrm{Ar}^{16} \mathrm{O}^{+}$interferences on

${ }^{56} \mathrm{Fe}$.[Szpunar, 2004; Lee et al., 2011] Oxygen gas was introduced into the sample carrier gas to mitigate the formation of reduced carbon species that leave carbon deposits and degrade instrument performance.[Hutton, 1986; Hausler, 1987] We used this method to investigate Fe ligand production in seawater and in culture media of the siderophore-producing cyanobacteria Synechococcus PCC sp. 7002. These applications demonstrate the utility of HPLC-ICP-MS for rapidly screening seawater samples for the presence of low concentration Fe ligands.

\subsection{Methods:}

2.2.1 Materials, standards, and reagents:

Ultra-high purity water $(18.2 \mathrm{M} \Omega \mathrm{cm})$, Optima grade methanol $(\mathrm{MeOH})$ and ammonium hydroxide $\left(\mathrm{NH}_{4} \mathrm{OH}\right)$ (Fisher scientific) were used in this study. For the analysis of standard compound sensitivity and low concentration $(<1 \mathrm{pM})$ naturally occurring ligands in seawater, the methanol was purified by sub boiling point distillation in a teflon still, which reduced $\mathrm{Fe}$ concentrations from $180 \mathrm{nM}$ to $2 \mathrm{nM}$ (as measured by ICP-MS).[Lee et al., 2011] Fe(III)ethylenediaminetetraacetic acid (Fe-EDTA) was obtained from Alfa Aesar as the monosodium salt. Ferric chloride $\left(\mathrm{FeCl}_{3} * 6 \mathrm{H}_{2} \mathrm{O}\right)$ was purchased from Fisher Scientific. Ferrioxamine E, desferrioxamine B (as deferoxamine mesylate salt), iron-free ferrichrome, Fe-heme (as hemin, 
from porcine), cyano-cobalamin (vitamin $\mathrm{B}_{12}$ ), nutrient salts and vitamins for culture media were obtained from Sigma Aldrich.

Stock solutions $(250 \mu \mathrm{M})$ of the standard compounds were made within 24 hours of analysis and stored at $4{ }^{\circ} \mathrm{C}$. Solutions of Fe-EDTA, ferrioxamine E, and cobalamin were prepared in water. Ferrioxamine $\mathrm{E}$ and ferrichrome stock solutions were prepared as free ligands in water to which a 1 molar equivalent of freshly made $1 \mathrm{mM} \mathrm{FeCl}_{3}$ solution was added. The Fe-heme solution was made by dissolving hemin in $1 \% \mathrm{NH}_{4} \mathrm{OH}$ under ultrasonication for 10 minutes and then diluting to $0.1 \% \mathrm{NH}_{4} \mathrm{OH}$ with $\mathrm{H}_{2} \mathrm{O}$.

Polytetrafluoroethylene (PTFE) or polycarbonate plastic ware was used for preparing standard solutions, culturing, seawater collection, and ligand extraction. Plastic ware was soaked overnight in $0.1 \%$ detergent (Citranox), rinsed $5 \mathrm{x}$ with $\mathrm{H}_{2} \mathrm{O}$ and then soaked in $1 \mathrm{~N} \mathrm{HCl}$ (J.T. Baker) for at least 2 days with final rinsing $5 \mathrm{x}$ with $\mathrm{H}_{2} \mathrm{O}$. Polyethersulfone and polycarbonate membrane filters $(0.2 \mu \mathrm{m})$ were soaked in $1 \mathrm{~N} \mathrm{HCl}$ overnight, rinsed once with $\mathrm{pH} 2$ water, and 5 times with $\mathrm{H}_{2} \mathrm{O}$. All samples for HPLC-ICP-MS analysis were placed in $2 \mathrm{~mL}$ amber glass autosampler vials or $250 \mu \mathrm{L}$ vial inserts (Agilent).

\subsubsection{Standard separation and isocratic standard injections:}

Stock solutions of $250 \mu \mathrm{M} \mathrm{Fe-EDTA}$, ferrioxamine E, ferrichrome, Fe-heme in $0.1 \%$ optima grade $\mathrm{NH}_{4} \mathrm{OH}$, and cobalamin were combined and diluted to prepare six concentrations of a standard mixture: $5 \mu \mathrm{M}, 2.5 \mu \mathrm{M}, 1.25 \mu \mathrm{M}$, and $0.05 \mu \mathrm{M}$. These solutions were sequentially analyzed by HPLC-ICP-MS. Quantification of the ICP-MS signal was based on peak area.

\subsubsection{Isocratic standard injections:}

Solutions of $250 \mu \mathrm{M}$ Fe-EDTA, ferrioxamine B, ferrioxamine E, ferrichrome, Fe-heme, and cobalamin were diluted to $5 \mu \mathrm{M}$ in $\mathrm{H}_{2} \mathrm{O}, 50 \% \mathrm{MeOH}$ in $\mathrm{H}_{2} \mathrm{O}$, or $100 \% \mathrm{MeOH}$. $0.1 \% \mathrm{NH}_{4} \mathrm{OH}$ was added to the $5 \mu \mathrm{M}$ Fe-heme solution to prevent precipitation. In order to monitor the effect 
of solvent on sensitivity, replicates of each solution were injected directly into the MC-ICP-MS without a column or flow splitter while pumping solvent with the same composition as the sample at a flow rate of $0.05 \mathrm{~mL} / \mathrm{min}$. Injections were repeated $2-4$ times to evaluate reproducibility. After switching the solvent composition, it took 40 minutes for the ICP-MS signal to stabilize under the new conditions. Quantification of the ICP-MS signal was based on peak area and normalized to the peak area of Fe-EDTA in $100 \% \mathrm{H}_{2} \mathrm{O}$.

\subsubsection{Seawater and culture samples:}

To test the sensitivity of the HPLC-ICP-MS method, organic compounds were extracted and analyzed from seawater and a marine cyanobacteria culture. Seawater was collected with an air driven PTFE deck pump from $100 \mathrm{~m}$ in the South Pacific Subtropical Gyre (26 ${ }^{\circ} 14.9^{\prime} \mathrm{S}$, 103 ${ }^{\circ} 57.6^{\prime} \mathrm{W}$; BiG-RAPA cruise, Nov 18 - Dec 14, 2010), filtered (0.2 $\left.\mu \mathrm{m}\right)$, and stored in the dark at room temperature in an acid washed barrel. Duplicate twenty liter samples were transferred into acid cleaned carboys, and one sample was spiked with $10 \mathrm{pM}$ of ferrichrome and ferrioxamine $\mathrm{E}$ to test the recovery and detection of siderophores with this method. The remaining seawater $(100 \mathrm{~L})$ was extracted and analyzed for the presence of naturally occurring ligands.

Synechococcus sp. PCC $7002(75 \mathrm{~mL})$ was grown under continuous light at $23^{\circ} \mathrm{C}$ in Trichodesmium media (3/4 Sargasso seawater diluted with $1 / 4 \mathrm{H}_{2} \mathrm{O}$ containing $1.5 \times 10^{-6} \mathrm{M}$ EDTA, $8 \times 10^{-6} \mathrm{M}$ phosphoric acid, $5 \times 10^{-8} \mathrm{M}$ ferric citrate, $10^{-7} \mathrm{M} \mathrm{MnSO}_{4}, 10^{-8} \mathrm{M} \mathrm{ZnCl}_{2}, 10^{-8} \mathrm{M}$ $\mathrm{NaMoO}_{4}, 10^{-10} \mathrm{M} \mathrm{CoCl}_{2}, 10^{-10} \mathrm{M} \mathrm{NiCl}_{2}, 10^{-10} \mathrm{M} \mathrm{NaSeO}_{3}$, and $1.5 \mu \mathrm{g}$ of cobalamin per liter)[Webb et al., 2001] plus $10^{-5} \mathrm{M} \mathrm{HNO}_{3}$. After seven days, three $1 \mathrm{~L}$ acid cleaned polycarbonate flasks containing $600 \mathrm{~mL}$ Trichodesmium media plus $10^{-5} \mathrm{M} \mathrm{HNO}_{3}$ without iron citrate were prepared. Two flasks were inoculated with equal amounts of the Synechococcus culture. The third flask was incubated alongside without inoculation as a media blank. After seven days, the culture media was centrifuged and filtered through a $0.2 \mu \mathrm{m}$ acid cleaned polycarbonate filter to remove the cells. The absence of heterotrophic contamination was confirmed using a marine purity broth $\left(8 \mathrm{~g} \mathrm{MgSO}_{4} \cdot 7 \mathrm{H}_{2} \mathrm{O}, 1.5 \mathrm{~g} \mathrm{CaCl}_{2} \cdot 2 \mathrm{H}_{2} \mathrm{O}, 20 \mathrm{~g} \mathrm{NaCl}, 17 \mathrm{~g}\right.$ AC Difco broth per liter of $\mathrm{H}_{2} \mathrm{O}$ ). 


\subsubsection{Solid phase organic extraction:}

The high salt concentration in seawater and marine culture media can interfere with ICP-MS analysis when salt crystals accumulate at the cone interface and reduce signal throughput (sensitivity). To prevent this as well as concentrate the sample, ligands were extracted from the filtered samples onto a polystyrene-divinylbenzene solid phase extraction column (Isolute, $\mathrm{ENV}+$, Biotage). This resin was selected based on its ability to bind a range of strong Fe ligands.[McCormack et al., 2003]

The columns were first rinsed with $5 \mathrm{~mL}$ of $\mathrm{MeOH}$ and $5 \mathrm{~mL}$ of $\mathrm{H}_{2} \mathrm{O}$. Then, the organic compounds from seawaters samples were extracted on a $1 \mathrm{~g}$ column at a flow rate of $8 \mathrm{~mL} / \mathrm{min}$, while compounds from Synechococcus media and media blank were extracted on a $0.5 \mathrm{~g}$ column at a flow rate of $6 \mathrm{~mL} / \mathrm{min}$. The columns were then rinsed with $25 \mathrm{~mL} \mathrm{H}_{2} \mathrm{O}$ to remove salts, and eluted with $5 \mathrm{~mL} \mathrm{MeOH}$. To determine the procedural blank associated with the extraction process, a $1 \mathrm{~g}$ resin column was rinsed with $5 \mathrm{~mL} \mathrm{MeOH}, 30 \mathrm{~mL}$ of $\mathrm{H}_{2} \mathrm{O}$, and then eluted with 5 $\mathrm{mL}$ of $\mathrm{MeOH}$. The SPE eluent from all samples was dried under high purity $\mathrm{N}_{2}$. The residue was dissolved in $0.5 \mathrm{~mL} \mathrm{H}_{2} \mathrm{O}$ and filtered through a $0.2 \mu \mathrm{m}$ polyvinylidene fluoride filter for analysis by HPLC-ICP-MS. All analyses were performed at least twice.

To quantify the recoveries of ferrioxamine $\mathrm{E}$ and ferrichrome from seawater, a three point calibration curve was created by analyzing three standard solutions containing $1 \mu \mathrm{M}, 0.5 \mu \mathrm{M}$ and $0.25 \mu \mathrm{M}$ of ferrichrome and ferrioxamine $\mathrm{E}$ dissolved in $\mathrm{H}_{2} \mathrm{O}$. Signal quantification was based on integrated peak areas from the HPLC-ICP-MS chromatogram. Coeluting organic carbon can potentially enhance the sensitivity of the ICP-MS towards Fe. To account for this matrix effect, we dissolved the solid phase extract from 20L of South Pacific seawater in a solution of $0.5 \mu \mathrm{M}$ ferrichrome and ferrioxamine E, and compared the ICP-MS response to the original (no dissolved extract) $0.5 \mu \mathrm{M}$ standard mixture. 


\subsubsection{High pressure liquid chromatography:}

HPLC separation was performed with a bioinert HPLC pump and auto-sampler (Agilent 1260 series) along with titanium or PEEK (polyether ether ketone) tubing to reduce background $\mathrm{Fe}$ levels. Samples ( $20 \mu \mathrm{L}$ volumes) were injected and separated on a PEEK C18 reverse-phase column $(250 \times 2.1 \mathrm{~mm}$ with $5 \mu \mathrm{m}$ particle size for the Synechococcus sp. 7002 culture, $150 \times 2.1$ $\mathrm{mm}$ with $3 \mu \mathrm{m}$ particle size for all other analyses, Hamilton) at a flow rate of $0.2 \mathrm{~mL} / \mathrm{min}$. Iron binding ligands were separated using a 20 minute linear gradient from $100 \% \mathrm{H}_{2} \mathrm{O}$ to $100 \%$ $\mathrm{MeOH}$ followed by a 10 minute isocratic elution with $\mathrm{MeOH}$. We tested the effect of $0.1 \%$, $0.01 \%$ and $0.001 \%$ formic acid (optima grade, Fisher Scientific) on the chromatographic separation of the standard compounds. The addition of formic acid increased the Fe background and did not significantly change the retention times of the standards, so we chose to exclude it from our mobile phases. A post-column PEEK flow splitter and a micro-metering valve (Upchurch Scientific) diverted 25\% of the eluent to the MC-ICP-MS and 75\% to a UV-Vis diode array detector and fraction collection (Fig. 1).

For the Synechococcus sp. PCC 7002 culture, the major Fe containing fractions were collected from five injections, dried under $\mathrm{N}_{2}$, dissolved in water, and analyzed by HPLC-ESI-MS (Agilent 6130 series) using the same column and solvent gradient described above.

\subsubsection{Interface between HPLC and ICP-MS:}

The use of organic solvents such as methanol can affect plasma stability, temperature, and shape and also promote the formation of reduced carbon species that interfere with ICP-MS sensitivity when they coat the cone interface. Three measures were taken to reduce the organic solvent interference: (1) lowering the flow rate entering the plasma to $50 \mu \mathrm{L} / \mathrm{min}$, (2) desolvation of the sample aerosol to remove most of the organic solvent, (3) addition of oxygen into the plasma to promote complete combustion of organic solvents to carbon dioxide. Desolvation was induced using an APEX Q inlet system (Elemental Scientific, Inc.) with a $50 \mu \mathrm{L} / \mathrm{min}$ quartz Micromist nebulizer. A heated macroporous PTFE membrane desolvator (Spiro TMD, Elemental Scientific) was coupled to the APEX outlet to ensure that most of the solvent was removed before it entered 
the plasma. Organic solvent combustion and Fe detection were optimized at a $50 \mathrm{~mL} / \mathrm{min}$ high purity $\mathrm{O}_{2}$ flow introduced post-Spiro TMD using a mass flow controller (Cole-Parmer).

\subsubsection{ICP-MS instrumentation:}

For trace metal analysis ${ }^{55} \mathrm{Mn},{ }^{56} \mathrm{Fe}$ and ${ }^{59} \mathrm{Co}$ were determined simultaneously on Faraday cups on a multi-collector magnetic sector ICP-MS (GV/Micromass IsoProbe) as described previously.[Lee et al., 2011] The instrument was equipped with X-sampler and X-skimmer cones (Spectron Inc.). Platinum cones are preferred over Nickel cones because they require less frequent cleaning when using $\mathrm{O}_{2}$ in the plasma. A hexapole collision cell with $\mathrm{Ar}$ and $\mathrm{H}_{2}$ collision gases was used to minimize ${ }^{40} \mathrm{Ar}^{16} \mathrm{O}^{+}$interferences on ${ }^{56} \mathrm{Fe}$ and to thermalize the ion beam. Operating conditions are listed in Table 1.

The ICP-MS was tuned daily with a $100 \mathrm{ppb} \mathrm{Mn}, \mathrm{Fe}$, Co and Ni solution dissolved in $0.2 \mathrm{~N}$ Vycor distilled $\mathrm{HNO}_{3}$ that was taken up by free aspiration with Teflon sipper tubing. Instrumental sensitivity was typically $2.0 \mathrm{~V}$ for ${ }^{56} \mathrm{Fe}$ and $3.55 \mathrm{~V}$ for ${ }^{59} \mathrm{Co}$.

\subsection{Results and Discussion:}

\subsubsection{Separation of standard compounds:}

To assess the chromatographic resolution, the limit of detection, and the linearity of the ICP-MS detector response, a mixture of 5 standard compounds was analyzed by HPLC-ICP-MS at four concentrations (100 pmol, 50 pmol, 25 pmol, and 1 pmol per $20 \mu \mathrm{L}$ injection). The standard mixture was composed of four Fe complexes (Fe-EDTA, ferrichrome, ferrioxamine E, and Feheme) and one cobalt complex (cobalamin) (Fig. 2). These compounds represent major classes of Fe ligands that may be present in seawater.

These compounds were separated with a gradient from water to methanol over 20 minutes. This gradient resulted in good peak resolution with little tailing. Four distinct peaks appear in the ${ }^{56} \mathrm{Fe}$ trace of the chromatogram (Fig. 2) with retention times of $2.1 \mathrm{~min}, 13.6 \mathrm{~min}, 15.2 \mathrm{~min}$, and 26.5 
min. These retention times correspond to Fe-EDTA, ferrichrome, ferrioxamine E, and Fe-heme respectively. A single peak appears in the ${ }^{59} \mathrm{Co}$ trace at $14.6 \mathrm{~min}$, corresponding to cobalamin.

Minimum detectable signals of the HPLC-ICP-MS measurements were calculated as three standard deviations above the mean background signal from the $\mathrm{H}_{2} \mathrm{O}$ blank. For ${ }^{56} \mathrm{Fe}$, the minimum detectable signal was $3.5 \times 10^{4} \mathrm{cps}$. This is 4-32 times higher than the peak heights from 1 pmol Fe-EDTA, ferrichrome, ferrioxamine E, and Fe-heme. In the case of ${ }^{59} \mathrm{Co}$, the limit was found to be $2.1 \times 10^{4}$ counts per second (cps), which is 31 times lower than the peak height from 1 pmol cobalamin. Based on these results, the detection limit of this method is in the range of 30250 femtomoles for Fe and Co complexes.

The ICP-MS signal for each standard compound varies linearly with standard concentration over the range of 1-100 pmol per $20 \mu \mathrm{L}$ with $\mathrm{R}^{2}$ values $>0.99$ (Table 2). The ICP-MS signal appears to decrease at later retention times, resulting in a lower sensitivity for Fe-Heme than for the other compounds. This suggests that solvent composition influences the ICP-MS sensitivity, as discussed below.

\subsubsection{Effects on ICP-MS sensitivity:}

To study the effect of solvent and organic ligand composition on the ${ }^{56} \mathrm{Fe}$ and ${ }^{59} \mathrm{Co}$ signal intensity during analysis, a series of standard compounds were injected directly into the ICP-MS with different mixtures of $\mathrm{H}_{2} \mathrm{O} / \mathrm{MeOH}$. The identity of the organic ligand appears to have little effect on the ICP-MS signal intensity. For each solvent condition, the mean ICPMS signal of each $\mathrm{Fe}$ compound deviates from the highest signal by 5 to $26 \%$. These variations are small compared to the difference observed between the signal from the same compound in methanol versus water. In general, the ICP-MS signal decreased by a factor of two as the proportion of $\mathrm{MeOH}$ increased from 0 to $100 \%$ (Fig. 3). Since Fe-heme elutes in $100 \% \mathrm{MeOH}$, this solvent effect on sensitivity may explain why the response factor for Fe-heme is always smaller than other Fe-complexes. 
There are several mechanisms that can explain this organic solvent effect. The nebulization and desolvation efficiency may vary for ligands in different solvents. Organics can also be a potential source of interferences or $\mathrm{C}^{+}$ion modifiers that can affect signal intensity.[Allain et al., 1991; AlAmmar et al., 1999; Kralj and Veber, 2003; Hu et al., 2004] Finally, organic solvents can influence the atomization and ionization of the analytes by cooling the central channel of the plasma.[Hu et al., 2004] In culture and seawater extract samples, there is an additional organic matrix effect that enhances sensitivity, discussed below.

\subsubsection{Fe ligand detection from seawater:}

Previous studies have reported siderophore concentrations of 3-20 pM in seawater from the North Atlantic.[Mawji et al., 2008] To evaluate the detection of Fe Ligands present in this concentration range using HPLC-ICPMS, 20 L of South Pacific surface seawater were spiked with $10 \mathrm{pM}$ of two hydroxamate siderophores. Organic compounds were then concentrated by solid phase extraction and brought up in $0.5 \mathrm{~mL} \mathrm{QH}_{2} \mathrm{O}$. The chromatogram of the extract containing the ferrichrome and ferrioxamine E spike contained two large peaks superimposed on the broad seawater background (Fig. 4a). We found that the organic matrix of the seawater extract enhances the sensitivity of the ICP-MS. By measuring the ICP-MS signal of $5 \mu \mathrm{M}$ ferrichrome and ferrioxamine $\mathrm{E}$ standards dissolved in $\mathrm{H}_{2} \mathrm{O}$ versus the same concentration dissolved in the seawater control extract, we calculated enhancement factors of 1.6 for ferrichrome and 1.5 for ferrioxamine E relative to the signal achieved in $\mathrm{H}_{2} \mathrm{O}$. This may occur through charge transfer reactions between $\mathrm{Fe}$ and $\mathrm{C}^{+}$-species formed in the plasma from organic compounds that co-elute with the siderophores.[Allain et al., 1991; Al-Ammar et al., 1999; Kralj and Veber, 2003; Hu et al., 2004] Recoveries for ferrichrome and ferrioxamine E were 56\% and $74 \%$ respectively, which is consistent with previously reported hydroxamate siderophore recoveries after solid phase extraction.[McCormack et al., 2003]

Other peaks also appear in the seawater sample between 10-20 minutes, suggesting the presence of a suite of naturally occurring Fe ligands at low concentrations. Analysis of the organic extract from South Pacific surface seawater with a longer gradient (0-50\% methanol over 30 minutes) reveals the presence of four major and several minor naturally occurring ligands that elute 
between 16 and 27 minutes (Fig 4b). Based on the calibration curves of the standard compounds, these peaks represent ligands that are present in seawater at 0.3 to $0.7 \mathrm{pM}$ concentrations.

\subsubsection{Synechococcus sp. PCC 7002:}

To further demonstrate the utility of HPLC-ICP-MS for detecting multiple strong iron ligands that are naturally produced in seawater, we analyzed culture media from Synechococcus sp. PCC 7002. Under low Fe conditions, this coastal cyanobacteria strain produces a suite of amphiphilic hydroxamate-type siderophores (synechobactin A-C) that have been previously isolated and structurally characterized.[Trick and Wilhelm, 1995; Wilhelm and Maxwell, 1996; Ito and Butler, 2005] These synechobactins can be extracted from spent culture media and detected by HPLCICP-MS.

The chromatogram for the Synechococcus sp. PCC 7002 media extract shows three large peaks with retention times of $26.7 \mathrm{~min}, 23.3 \mathrm{~min}$, and $18.7 \mathrm{~min}$ (Fig. 5, peaks f, h, and i). The fractions containing these peaks were collected and analyzed by HPLC-ESI-MS. They yielded spectra with parent ions at masses that correspond to the Fe form of synechobactins $\mathrm{A}\left(\mathrm{C}_{26} \mathrm{H}_{46} \mathrm{~N}_{4} \mathrm{O}_{9} \mathrm{Fe}^{+}=\right.$ $614.3 \mathrm{~m} / \mathrm{z}), \mathrm{B}\left(\mathrm{C}_{24} \mathrm{H}_{42} \mathrm{~N}_{4} \mathrm{O}_{9} \mathrm{Fe}^{+}=586.2\right)$, and $\mathrm{C}\left(\mathrm{C}_{26} \mathrm{H}_{46} \mathrm{~N}_{4} \mathrm{O}_{9} \mathrm{Fe}^{+}=558.2\right)$ respectively. [Ito and Butler, 2005] In addition to the three major peaks, there is a small peak at $3.2 \mathrm{~min}$ (Fig. 5, peak a) that also appears in the blank media extract and which may correspond to uncomplexed Fe, Fe-EDTA or Fe-citrate used in the culture medium to buffer free ion concentrations, or from the $\mathrm{ENV}+$ resin. There are also additional peaks with retention times at $21.0 \mathrm{~min}, 14.8 \mathrm{~min}, 11.58$ min, $10.58 \mathrm{~min}$, and $9.37 \mathrm{~min}$ that indicate the presence of uncharacterized Fe ligands (Fig. 5, peaks b-e, g).

The presence of uncharacterized Fe ligands in the HPLC-ICP-MS chromatograms of seawater and Synechococcus sp. PCC 7002 extract demonstrates the strength of this method for screening marine samples for Fe ligands. As in the original studies that characterized synechobactins A-C, siderophores are commonly detected in cell culture media using spectrophotometric tests such as the chrome azurol S assay, which has a detection limit of $2 \mathrm{nmol}$ of free ligand.[Schwyn and Neilands, 1987; Hasegawa et al., 2004] Since HPLC-ICP-MS has a lower detection limit and 
measures the complexed rather than free form of the ligand, this method can detect the presence of strong metal ligands that cannot be detected by spectrophotometric assays.

\subsection{Conclusions:}

The determination of Fe ligands in seawater requires a targeted approach to better understand ligand diversity and variability. As a first step to identify target complexes, HPLC-ICP-MS is ideal for rapidly screening seawater and marine cultures for the presence of distinct Fe ligands. While precise quantification based on this method is still hindered by sensitivity changes due to mobile phase composition and organic matrix effects, it is capable of estimating ligand concentrations within a factor of two. The major components can then be isolated and structural characterization with complimentary techniques such as ESI-MS analyses.

\subsection{Acknowledgments:}

We would like to thank John Waterbury for the Synechococcus sp. PCC 7002 culture. James Moffett, Carl Lamborg, Philip Gschwend, Mak Saito, and Chris Follett provided helpful discussion that improved this manuscript. We would also like to thank Reinhard Niessner and three anonymous reviewers for their constructive feedback. This work was supported by the National Science Foundation Graduate Research Fellowship Program (NSF Award 0645960), NSF Award OCE-01751733, NSF Science and Technology Center Award IF0424599 administered through the Center for Microbial Oceanography Research and Education (CMORE), and the Gordon and Betty Moore Foundation.

\subsection{References:}

Al-Ammar, A. S., E. Reitznerová, and R. M. Barnes (1999), Feasibility of using beryllium as internal reference to reduce non-spectroscopic carbon species matrix effect in the inductively coupled plasma-mass spectrometry (ICP-MS) determination of boron in biological samples, Spectrochim. Acta Part B, (54), 1813-1820.

Allain, P., L. Jaunault, Y. Mauras, and J. Mermet (1991), Signal enhancement of elements due to the presence of carbon-containing compounds in inductively coupled plasma mass spectrometry, Anal. Chem., 63, 1497-1498. 
Ammann, A. A. (2002), Speciation of heavy metals in environmental water by ion chromatography coupled to ICP-MS., Anal. Bioanal. Chem., 372(3), 448-52, doi:10.1007/s00216-001-1115-8.

De Baar, H., P. Boyd, K. Coale, and M. Landry (2005), Synthesis of iron fertilization experiments: From the Iron Age in the Age of Enlightenment, J. Geophys. Res, 110(C9), 124, doi:10.1029/2004JC002601.

Boyd, P. W., and M. J. Ellwood (2010), The biogeochemical cycle of iron in the ocean, Nat. Geosci., 3(10), 675-682, doi:10.1038/ngeo964.

Boyd, P. W. et al. (2007), Mesoscale Iron Enrichment Experiments 1993-2005: Synthesis and Future Directions, Science, 315(February), 612-617.

Boye, M., and C. M. G. van Den Berg (2000), Iron availability and the release of ironcomplexing ligands by Emiliania huxleyi, Mar. Chem., 70(4), 277-287, doi:10.1016/S03044203(00)00032-3.

Boye, M., C. M. G. van den Berg, J. de Jong, H. Leach, P. Croot, and H. J. W. de Baar (2001), Organic complexation of iron in the Southern Ocean, Deep Sea Res. Part I Oceanogr. Res. Pap., 48(6), 1477-1497, doi:10.1016/S0967-0637(00)00099-6.

Bruland, K. W., J. R. Donat, and D. A. Hutchins (1991), Interactive influences of bioactive trace metals on biological production in oceanic waters, Limnol. Oceanogr., 36(8), 1555-1577, doi:10.4319/1o.1991.36.8.1555.

Buck, K. N., K. E. Selph, and K. A. Barbeau (2010), Iron-binding ligand production and copper speciation in an incubation experiment of Antarctic Peninsula shelf waters from the Bransfield Strait, Southern Ocean, Mar. Chem., 122(1-4), 148-159, doi:10.1016/j.marchem.2010.06.002.

Gledhill, M., and K. N. Buck (2012), The organic complexation of iron in the marine environment: a review., Front. Microbiol., 3, 1-17, doi:10.3389/fmicb.2012.00069.

Gledhill, M., P. McCormack, S. Ussher, E. P. Achterberg, R. F. C. Mantoura, and P. J. Worsfold (2004), Production of siderophore type chelates by mixed bacterioplankton populations in nutrient enriched seawater incubations, Mar. Chem., 88(1-2), 75-83, doi:10.1016/j.marchem.2004.03.003.

Harrington, C. F., S. Elahi, S. A. Merson, and P. Ponnampalavanar (2001), A method for the quantitative analysis of iron speciation in meat by using a combination of spectrophotometric methods and high-performance liquid chromatography coupled to sector field inductively coupled plasma mass spectrometry, Anal. Chem., 73(18), 4422-4427.

Hasegawa, H., T. Maki, K. Asano, K. Ueda, and K. Ueda (2004), Detection of iron(III)-binding 
ligands originating from marine phytoplankton using cathodic stripping voltammetry., Anal. Sci., 20(1), 89-93.

Hausler, D. (1987), Trace element analysis of organic solutions using inductively coupled plasma-mass spectrometry, Spectrochim. Acta, 42B(1987), 63-73.

Heumann, K. G., L. Rottmann, and J. Vogl (1994), Elemental speciation with liquid chromatography-inductively coupled plasma isotope dilution mass spectrometry, J. Anal. At. Spectrom., 9(12), 1351-1355.

Hopkinson, B. M., and K. A. Barbeau (2012), Iron transporters in marine prokaryotic genomes and metagenomes., Environ. Microbiol., 14(1), 114-28, doi:10.1111/j.14622920.2011.02539.x.

Hopkinson, B. M., and F. M. M. Morel (2009), The role of siderophores in iron acquisition by photosynthetic marine microorganisms., Biometals, 22(4), 659-69, doi:10.1007/s10534009-9235-2.

$\mathrm{Hu}, \mathrm{Z}$., S. Hu, S. Gao, Y. Liu, and S. Lin (2004), Volatile organic solvent-induced signal enhancements in inductively coupled plasma-mass spectrometry: a case study of methanol and acetone, Spectrochim. Acta, 59, 1463-1470, doi:10.1016/j.sab.2004.07.007.

Hutton, R. C. (1986), Application of inductively Coupled Plasma Source Mass Spectrometry ( ICP-MS ) to the Determination of Trace Metals in Organics, J. Anal. At. Spectrom., l(August), 259-263.

Ito, Y., and A. Butler (2005), Structure of synechobactins, new siderophores of the marine cyanobacterium Synechococcus sp. PCC 7002, Limnol. Oceanogr., 50(6), 1918-1923, doi:10.4319/lo.2005.50.6.1918.

Jickells, T. D. et al. (2005), Global iron connections between desert dust, ocean biogeochemistry, and climate, Science, 308(67), 67-71, doi:10.1126/science.1105959.

Kralj, P., and M. Veber (2003), Investigations into nonspectroscopic effects of organic compounds in inductively coupled plasma mass spectrometry, Acta Chim. Slov., 50, 633644.

Leão, P. N., M. T. S. D. Vasconcelos, and V. M. Vasconcelos (2007), Role of marine cyanobacteria in trace metal bioavailability in seawater., Microb. Ecol., 53(1), 104-9, doi:10.1007/s00248-006-9153-6.

Lechtenfeld, O. J., B. P. Koch, W. Geibert, K. U. Ludwichowski, and G. Kattner (2011), Inorganics in Organics: Quantification of Organic Phosphorus and Sulfur and Trace Element Speciation in Natural Organic Matter Using HPLC-ICPMS, Anal. Chem., 89688974. 
Lee, J. M. J. M., E. A. E. A. Boyle, Y. Echegoyen-Sanz, J. N. J. N. Fitzsimmons, R. Zhang, and R. A. R. A. Kayser (2011), Analysis of trace metals ( $\mathrm{Cu}, \mathrm{Cd}, \mathrm{Pb}$, and $\mathrm{Fe})$ in seawater using single batch nitrilotriacetate resin extraction and isotope dilution inductively coupled plasma mass spectrometry., Anal. Chim. Acta, 686(1-2), 93-101, doi:10.1016/j.aca.2010.11.052.

Lis, H., and Y. Shaked (2009), Probing the bioavailability of organically bound iron: a case study in the Synechococcus-rich waters of the Gulf of Aqaba, Aquat. Microb. Ecol., 56(September), 241-253, doi:10.3354/ame01347.

Malmstrom, R. R., S. Rodrigue, K. H. Huang, L. Kelly, S. E. Kern, A. Thompson, S. Roggensack, P. M. Berube, M. R. Henn, and S. W. Chisholm (2013), Ecology of uncultured Prochlorococcus clades revealed through single-cell genomics and biogeographic analysis., ISME J., 7, 184-198, doi:10.1038/ismej.2012.89.

Mawji, E., M. Gledhill, J. A. Milton, G. A. Tarran, S. Ussher, A. Thompson, G. A. Wolff, P. J. Worsfold, and E. P. Achterberg (2008), Hydroxamate siderophores: occurrence and importance in the Atlantic Ocean., Environ. Sci. Technol., 42(23), 8675-80.

Mawji, E., M. Gledhill, J. A. Milton, M. V. Zubkov, A. Thompson, G. A. Wolff, and E. P. Achterberg (2011), Production of siderophore type chelates in Atlantic Ocean waters enriched with different carbon and nitrogen sources, Mar. Chem., 124(1-4), 90-99, doi:10.1016/j.marchem.2010.12.005.

McCormack, P., P. J. Worsfold, and M. Gledhill (2003), Separation and detection of siderophores produced by marine bacterioplankton using high-performance liquid chromatography with electrospray ionization mass spectrometry., Anal. Chem., 75(11), 2647-52.

Morel, F. M. M., and N. M. Price (2003), The biogeochemical cycles of trace metals in the oceans., Science, 300(5621), 944-7, doi:10.1126/science.1083545.

Morel, F. M. M., A. J. Milligan, and M. A. Saito (2003), Marine Bioinorganic Chemistry: The Role of Trace Metals in the Oceanic Cycles of Major Nutrients, Treatise Geochemistry, Vol. 6.

Morel, F. M. M., A. B. Kustka, and Y. Shaked (2008), The role of unchelated Fe in the iron nutrition of phytoplankton, Limnol. Oceanogr., 53(1), 400-404.

Rivers, A. R. (2009), Iron limitation and the role of siderophores in marine Synechococcus, Massachusetts Institute of Technology.

Rottmann, L., and K. G. Heumann (1994), Determination of heavy metal interactions with dissolved organic materials in natural aquatic systems by coupling a high-performance liquid chromatography system with an inductively coupled plasma mass spectrometer, Anal. 
Chem., 66(21), 3709-3715.

Rue, E., and K. Bruland (1995), Complexation of iron(III) by natural organic ligands in the Central North Pacific as determined by a new competitive ligand equilibration/adsorptive cathodic stripping voltammetric method, Mar. Chem., 50(1-4), 117-138, doi:10.1016/03044203(95)00031-L.

Sandy, M., and A. Butler (2009), Microbial iron acquisition: marine and terrestrial siderophores., Chem. Rev., 109(10), 4580-95, doi:10.1021/cr9002787.

Schwyn, B., and J. B. Neilands (1987), Universal chemical assay for the detection and determination of siderophores., Anal. Biochem., 160(1), 47-56.

Shaked, Y., and H. Lis (2012), Disassembling iron availability to phytoplankton., Front. Microbiol., 3(April), 123, doi:10.3389/fmicb.2012.00123.

Szpunar, J. (2004), Metallomics: a new frontier in analytical chemistry., Anal. Bioanal. Chem., 378(1), 54-6, doi:10.1007/s00216-003-2333-z.

Thompson, A. W., K. Huang, M. a Saito, and S. W. Chisholm (2011), Transcriptome response of high- and low-light-adapted Prochlorococcus strains to changing iron availability., ISME J., 9313, 1-15, doi:10.1038/ismej.2011.49.

Trick, C., and S. Wilhelm (1995), Physiological changes in the coastal marine cyanobacterium Synechococcus sp. PCC 7002 exposed to low ferric ion levels, Mar. Chem., 50(1-4), 207217, doi:10.1016/0304-4203(95)00036-Q.

Velasquez, I., B. L. Nunn, E. Ibisanmi, D. R. Goodlett, K. A. Hunter, and S. G. Sander (2011), Detection of hydroxamate siderophores in coastal and Sub-Antarctic waters off the South Eastern Coast of New Zealand, Mar. Chem., 126(1-4), 97-107, doi:10.1016/j.marchem.2011.04.003.

Vogl, J., and K. G. Heumann (1997), Determination of heavy metal complexes with humic substances by HPLC/ICP-MS coupling using on-line isotope dilution technique, Fresenius. J. Anal. Chem., 359(4-5), 438-441, doi:10.1007/s002160050606.

Vogl, J., and K. G. Heumann (1998), Development of an ICP-IDMS Method for Dissolved Organic Carbon Determinations and Its Application to Chromatographic Fractions of Heavy Metal Complexes with Humic Substances, Anal. Chem., 70(10), 2038-2043, doi:10.1021/ac971283p.

Vraspir, J. M., and A. Butler (2009), Chemistry of Marine Ligands and Siderophores, Ann. Rev. Mar. Sci., 1(1), 43-63, doi:10.1146/annurev.marine.010908.163712.

Webb, E. A., J. W. Moffett, and J. B. Waterbury (2001), Iron Stress in Open-Ocean Cyanobacteria (Synechococcus, Trichodesmium, and Crocosphaera spp.): Identification of 
the IdiA Protein, Appl. Environ. Microbiol., 67(12), 5444-5452, doi:10.1128/AEM.67.12.5444.

Wilhelm, S., and D. Maxwell (1996), Growth, iron requirements, and siderophore production in iron-limited Synechococcus PCC 7002, Limnol. Oceanogr., 41(1), 89-97.

$\mathrm{Wu}$, J., and G. Luther (1995), Complexation of Fe(III) by natural organic ligands in the Northwest Atlantic Ocean by a competitive ligand equilibration method and a kinetic approach, Mar. Chem., 50(1-4), 159-177, doi:10.1016/0304-4203(95)00033-N. 


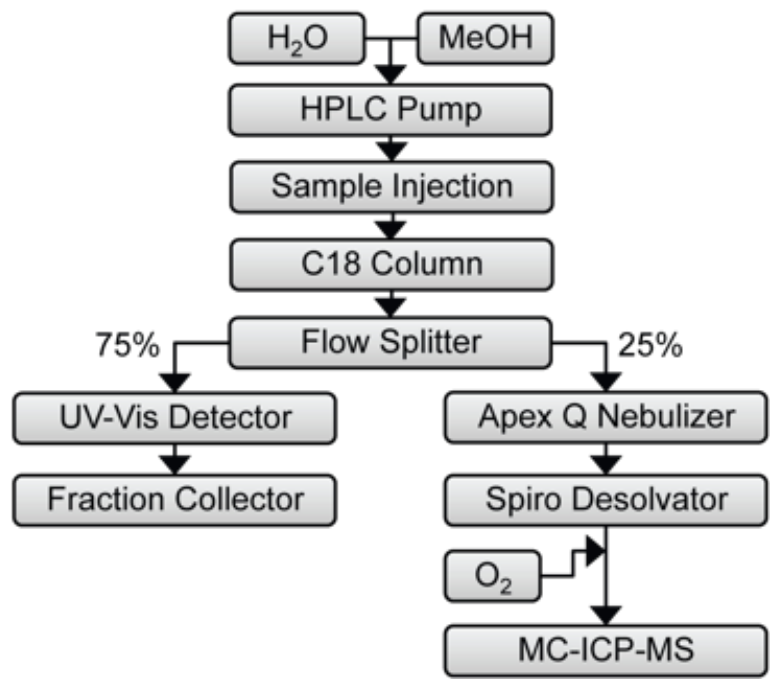

Figure 2.1: Schematic of high pressure liquid chromatography - inductively coupled plasma mass spectrometry (HPLC-ICP-MS) instrumentation and workflow. Compounds are separated by HPLC with a gradient from $\mathrm{H}_{2} \mathrm{O}$ to $\mathrm{MeOH}$. Twenty five percent of the eluent from the HPLC column is nebulized, desolvated, and analyzed by ICPMS. Oxygen is added to the sample carrier gas before entering the ICP-MS to mitigate incomplete combustion of organic carbon in the plasma. 
Table 2.1: Operating conditions for HPLC-ICP-

MS analysis.

HPLC settings

Mobile phase A

$\mathrm{H}_{2} \mathrm{O}$

Mobile phase B

$\mathrm{MeOH}$

Flow rate

$0.2 \mathrm{~mL} / \mathrm{min}$

Column

C18

Injection Volume

$20 \mu \mathrm{L}$

ICP-MS settings

Argon flow through APEX

$3 \mathrm{~L} \mathrm{~min}^{-1}$

APEX spray chamber temperature

$140{ }^{\circ} \mathrm{C}$

APEX condensation temperature

$2{ }^{\circ} \mathrm{C}$

Oxygen gas flow

$50 \mathrm{~mL} \mathrm{~min}^{-1}$

Forward power

$1350 \mathrm{~W}$

Reflected power

$<1 \mathrm{~W}$

Hexapole Ar gas flow

$1.8 \mathrm{~mL} \mathrm{~min}^{-1}$

Hexapole $\mathrm{H}_{2}$ gas flow

$2.5 \mathrm{~mL} \mathrm{~min}^{-1}$

Expansion pressure

$3 \times 10^{-1}$ mbar

Hexapole pressure

$4 \times 10^{-4}$ mbar

Analyzer pressure

$2 \times 10^{-7}$ mbar

Nebulizer gas flow

$0.7 \mathrm{~L} \mathrm{~min}^{-1}$

Cool gas flow

$14 \mathrm{~L} \mathrm{~min}^{-1}$

Aux. gas flow

1.0 $\mathrm{L} \mathrm{min}^{-1}$

Integration time

$2 \mathrm{sec}$ 


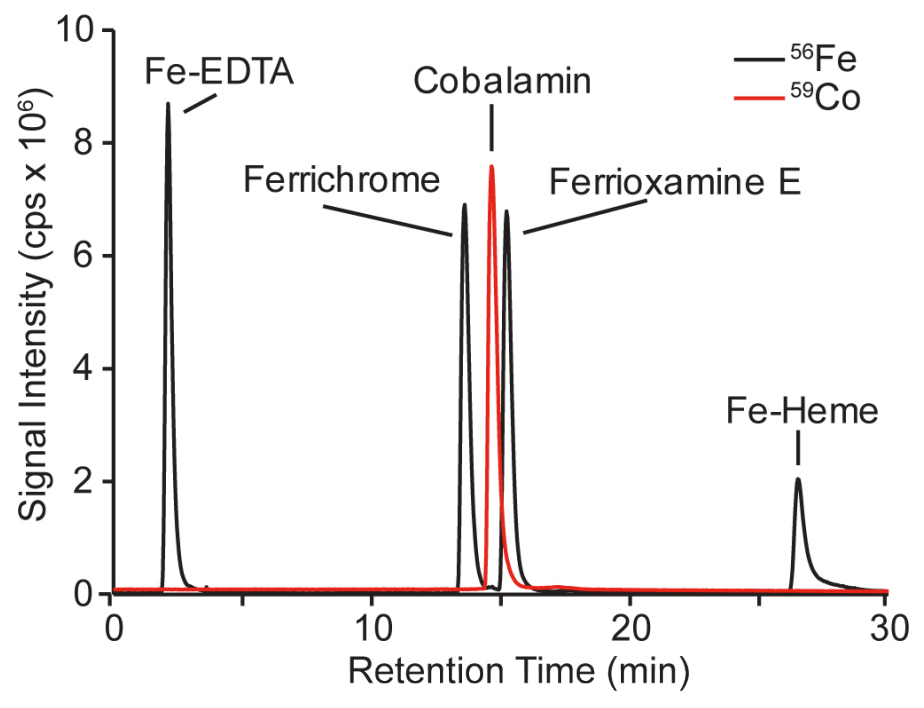

Figure 2.2: Separation of Fe-EDTA, ferrichrome, cobalamin, ferrioxamine E, Fe-heme ( 25 pmol) by HPLC-ICPMS. ICP-MS signal is in detector counts per second (cps). The chromatography gives sharp, well-resolved peaks for each standard compound with little tailing. There is some loss of signal intensity with increasing mobile phase methanol content. 
Table 2.2: Retention time, linear sensitivity, and correlation coefficient of standard compound concentration vs. ICP-MS signal

\begin{tabular}{llll}
\hline Compound & $\begin{array}{l}\text { Retention } \\
\text { time (min) }\end{array}$ & $\begin{array}{l}\text { Sensitivity } \mathbf{( 1 0}^{\mathbf{1 2}} \\
\left.\mathbf{c p s} \mathbf{m i n}^{*} \mathbf{M}^{\mathbf{1}}\right)\end{array}$ & $\mathbf{R}^{\mathbf{2}}$ \\
\hline Fe-EDTA & 2.1 & $3.1 \pm 0.2$ & 0.990 \\
Ferrichrome & 13.6 & $3.3 \pm 0.06$ & 0.999 \\
Ferrioxamine E & 15.2 & $3.4 \pm 0.1$ & 0.998 \\
Fe-heme & 26.5 & $2.5 \pm 0.2$ & 0.987 \\
Cobalamin & 14.6 & $4.0 \pm 0.09$ & 0.999
\end{tabular}




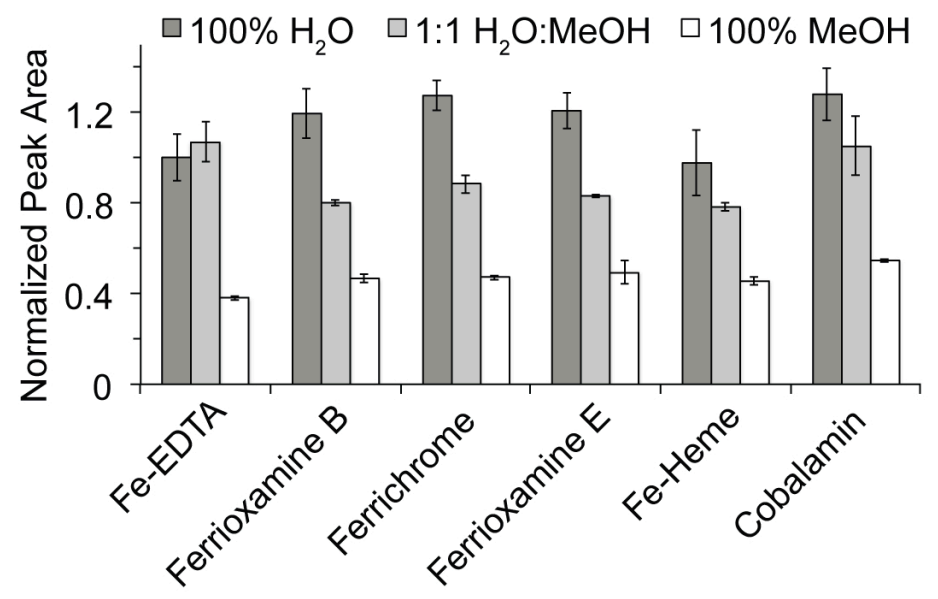

Figure 2.3: Effect of solvent composition on ICP-MS signal from 100 pmol of six standard compounds. Error bars represent $\pm 1 \sigma$. The trace metal response factor decreases by two-fold as the proportion of $\mathrm{MeOH}$ increases from 0 $100 \%$. 


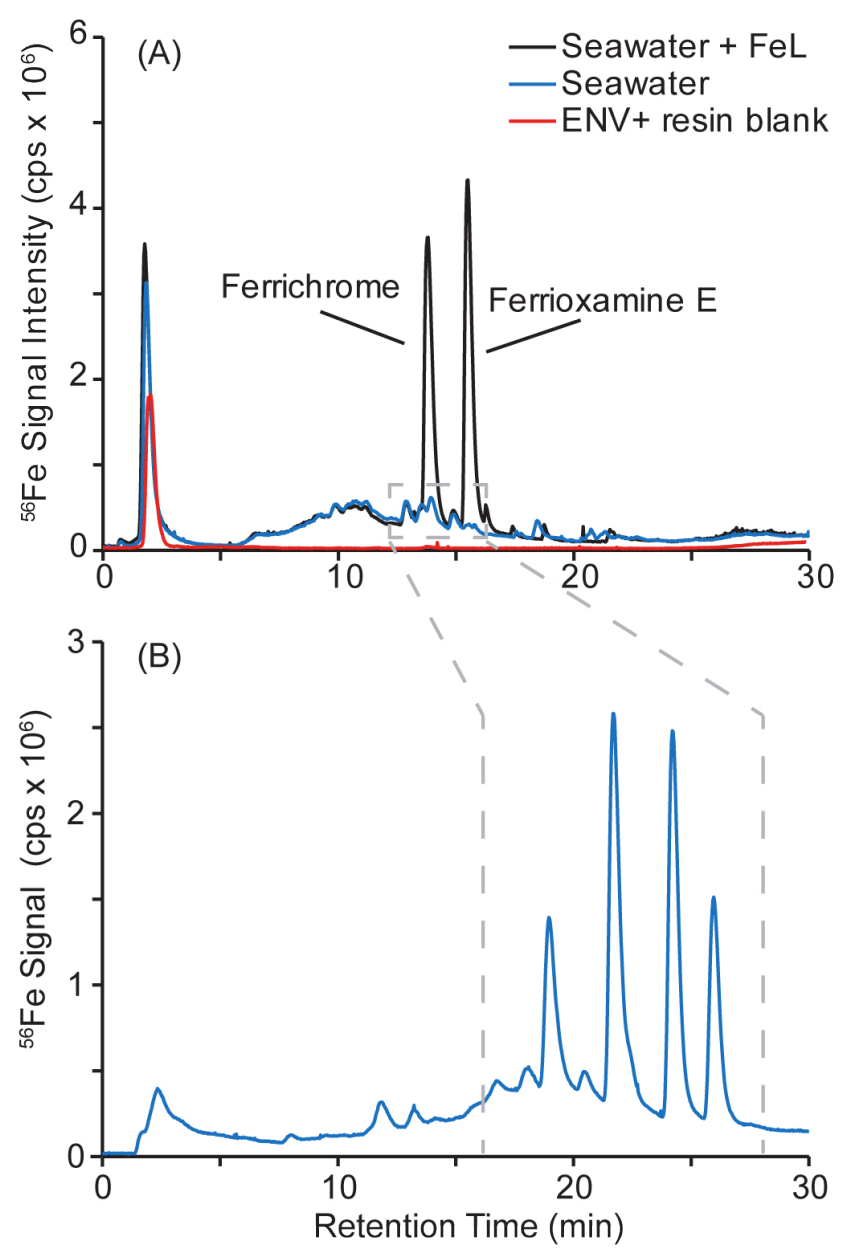

Figure 2.4: (A) Organic extract from 20L of South Pacific Seawater spiked with 10pM ferrichrome and ferrioxamine $\mathrm{E}$ was separated by HPLC with a gradient from $0-100 \%$ methanol over 20 minutes. The two tall peaks between 10-20 minutes correspond to the added siderophores, while smaller peaks that are also present in the seawater control indicate naturally occurring Fe ligands. (B) To obtain better resolution of the naturally occurring ligands, the organic extract of $100 \mathrm{~L}$ of South Pacific seawater was separated with a $0-50 \%$ methanol gradient over 30 minutes. Trace metal clean distilled methanol was used for the separation in (B). The gray dashed lines highlight the regions containing major naturally occurring organic ligands. 


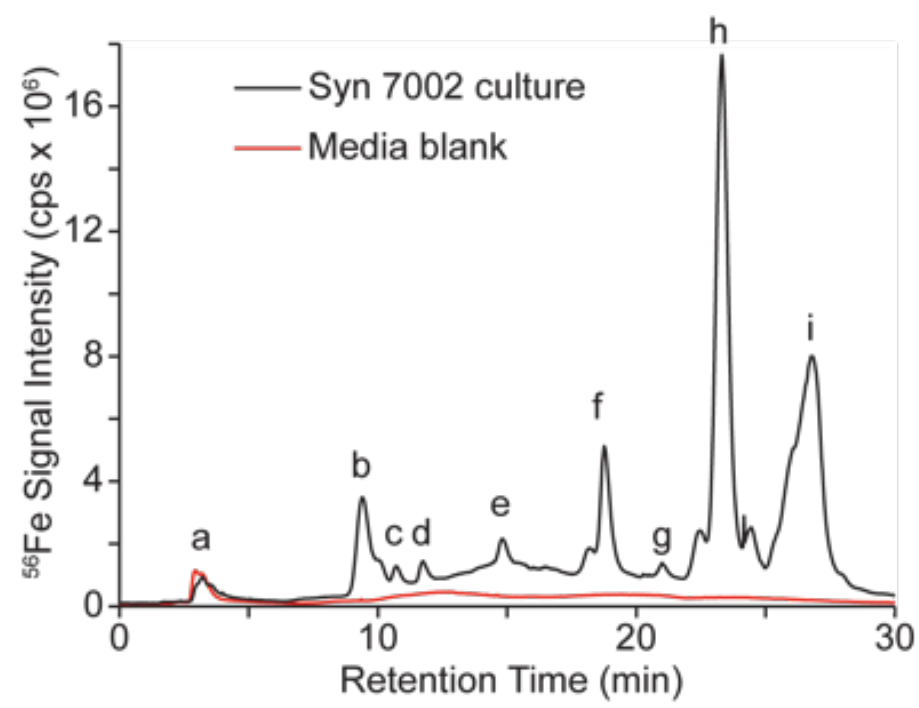

Figure 2.5: Separation of organic extract from a Synechococcus sp. PCC 7002 culture. The ${ }^{56} \mathrm{Fe}$ peaks $\mathbf{f}, \mathbf{h}$, and $\mathbf{i}$ correspond to the three Synechobactins previously characterized by Ito and Butler (2005). The other peaks (b-e, g) indicate the existence of unidentified compounds produced under low Fe conditions. 


\section{Appendix 2.1 Characterization of iron binding ligands from Prochlorococcus}

Marine organisms produce a wide array of organic ligands with a strong affinity for iron. These ligands may serve many functions, including Fe transport, uptake, storage, and detoxification, and their release from cells is a significant source of the iron binding ligands found in seawater [Boye and van Den Berg, 2000; Sandy and Butler, 2009; Vraspir and Butler, 2009; Buck et al., 2010; Gledhill and Buck, 2012]. Marine picocyanobacteria are of particular interest since they are the most abundant photosynthetic organisms on the planet and they dominate primary productivity in Fe limited regions. Any iron ligands produced by marine picocyanobacteria are likely to be important to marine iron cycling. However, iron binding ligands from the major marine cyanobacteria Prochlorococcus have not been characterized.

Preliminary data using LC-ICPMS screening techniques indicates that most strains of Prochlorococcus tested thus far are capable of producing iron ligands (Fig. A2.1). The retention times of these compounds are variable, suggesting that strains may produce different ligand suites. In addition, iron ligands are absent from methanol extracts of Prochlorococcus cells, suggesting that they may be exuded rather than released from lysed cells. The production of these compounds does not appear to be linked to iron limitation (Figure A2.2), but rather scales with overall cell growth. These compounds were stable on frozen SPE columns (Biotage ENV+), but degraded within days upon elution in $\mathrm{MeOH}$ and storage at $4^{\circ} \mathrm{C}$. Numerous attempts were made to purify the compounds for MS or NMR analysis but the compound instability remained an issue. Future work will focus on the determination of the compound masses by high resolution LC-ESIMS on a freshly eluted sample. These compounds were not detected in cultures of Synechococcus WH 8102.

It is possible that the release of iron-binding compounds by Prochlorococcus may impact iron bioavailability. Estimates of uncomplexed iron concentrations in iron limited regions are often too low to possibly support the growth of these organisms [Morel et al., 2008; Thompson et al., 2011], suggesting that they are able to access the organically complexed Fe dissolved in surface waters. Furthermore, several clades of Prochlorococcus, including Prochlorococcus 9202, possess Ton-dependent siderophore transporters that are upregulated under conditions of iron stress [Malmstrom et al., 2013]. Other studies have suggested that cyanobacteria may reduce free 
iron from strong complexing ligands prior to uptake, although few $\mathrm{Fe}^{2+}$ transport genes are found in picocyanobacteria genomes [Hopkinson and Barbeau, 2012; Shaked and Lis, 2012]. Some unbound siderophores have been shown to prevent the growth of Synechococcus strains, possibly by interfering with reductive uptake mechanisms or by entering the cell and competing for Fe from essential enzymes [Lis and Shaked, 2009; Rivers, 2009; Shaked and Lis, 2012]. Furthermore, different Prochlorococcus strains have distinct responses to low iron concentrations, suggesting multiple adaptive strategies to iron stress (Fig A2.3). It has yet to be definitively shown whether organically complexed iron is accessible to marine picocyanobacteria. The results presented here provide preliminary evidence that the release of iron binding metabolites could potentially play a role.

\section{Methods:}

1L cultures of Prochlorococcus strains were grown at $20^{\circ} \mathrm{C}$ in Pro99 media in uv oxidized or solid phase extracted (ENV+ resin, Biotage) seawater (to remove dissolved organic compounds). Nutrient stocks were passed through cleaned Chelex-100 resin to remove metal contamination. Growth was monitored by fluorescence (ex440 em680) daily using a Bio-Tek Synergy HT plate reader. During late-log growth ( $\sim 7$ days for most strains), the media was centrifuged to remove cells, filtered through a $0.2 \mathrm{um}$ sterivex polyethersulfone filter, and extracted onto an ENV+ resin column (1g, 6mL, Biotage). The columns were rinsed with $\mathrm{qH}_{2} \mathrm{O}$ to remove salts and compounds were eluted with $6 \mathrm{~mL} \mathrm{MeOH}$. The methanol extract was concentrated under an $\mathrm{N}_{2}$ stream to $1 \mathrm{~mL}$, and the resulting extract was analyzed by LC-ICPMS as described above. 

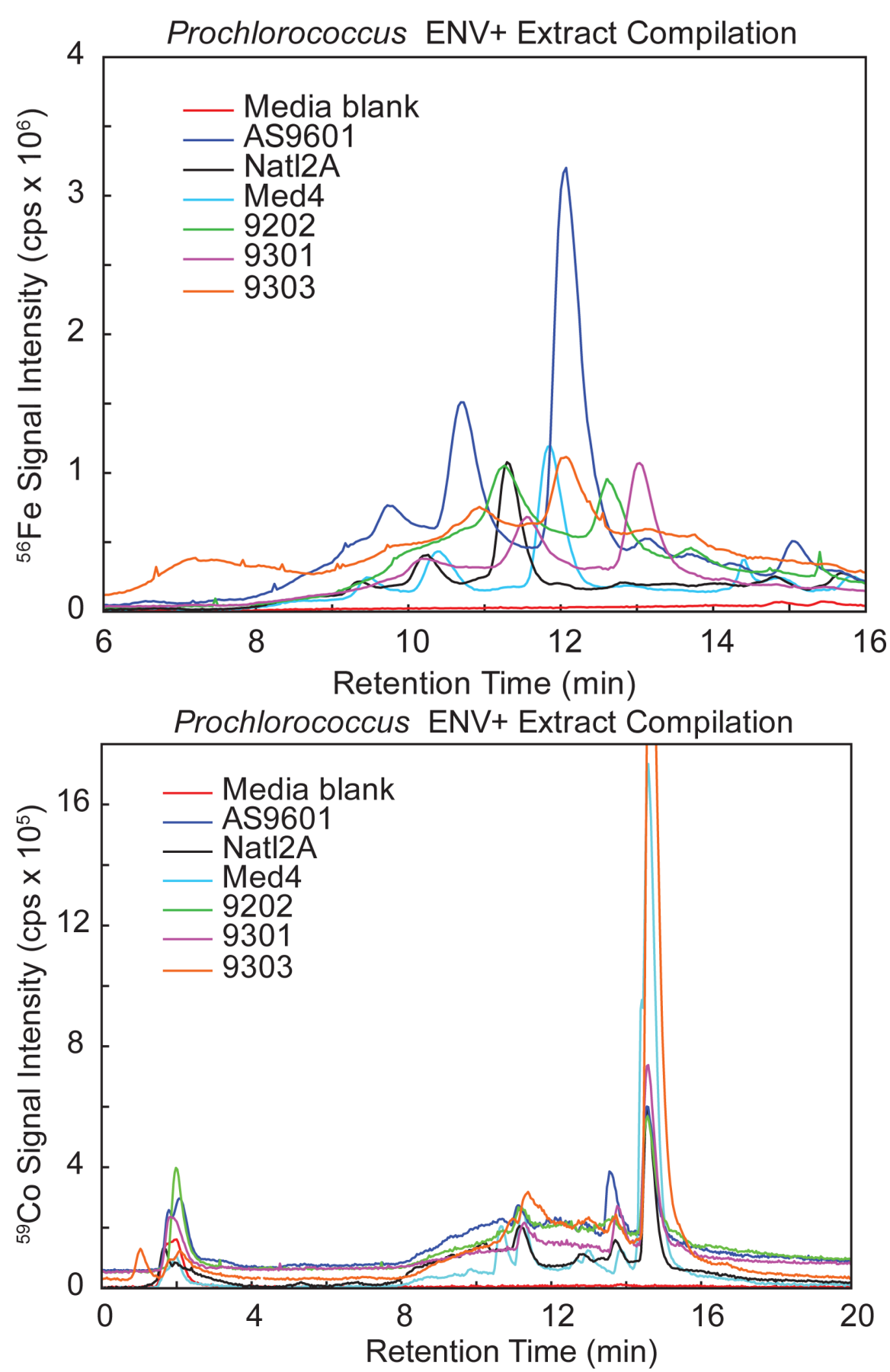

Figure A2.1: Compilation of LC-ICPMS chromatograms of growth media extract from axenic Prochlorococcus cultures for ${ }^{56} \mathrm{Fe}$ (top) and ${ }^{59} \mathrm{Co}$ (bottom). Most strains produces two or more iron ligands (indicated by peaks). All strains also produce pseudocobalamin (large peak in bottom chromatogram, confirmed by LC-ESIMS, $672.776 \mathrm{~m} / \mathrm{z}$ ). 


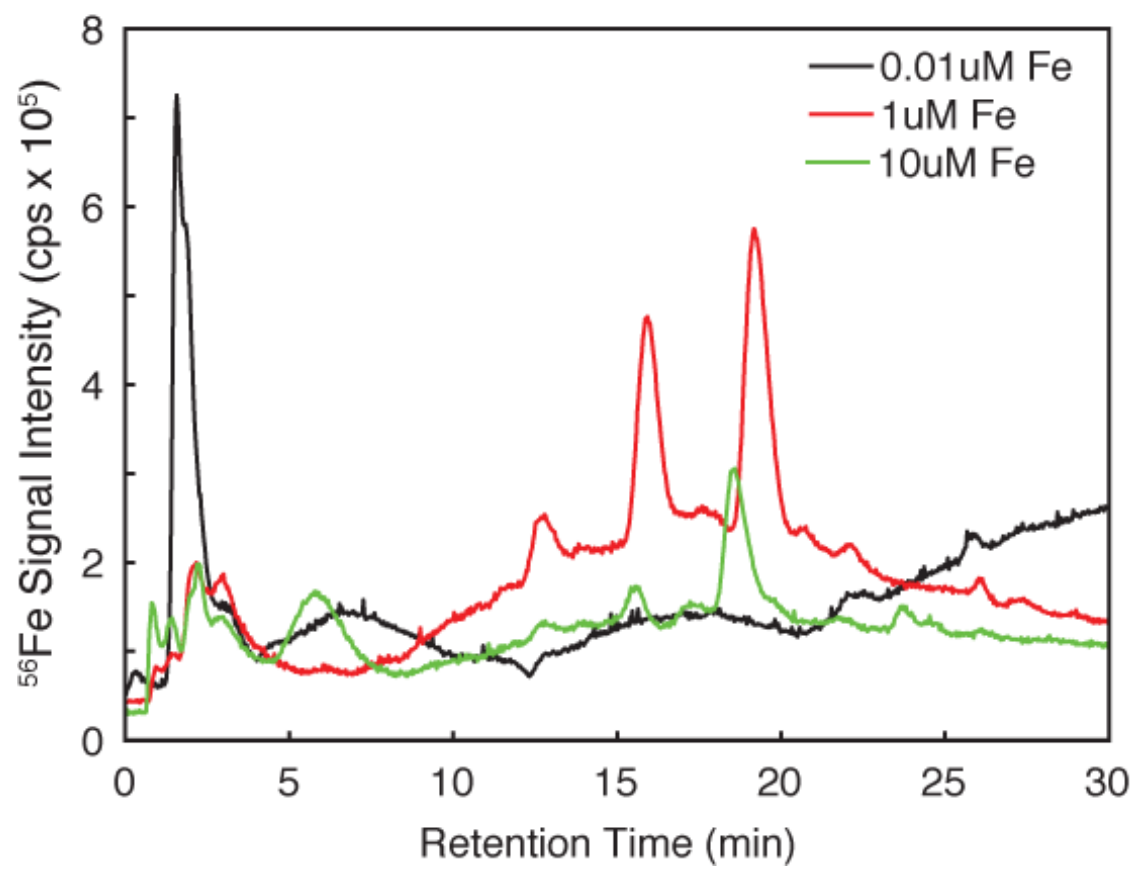

Figure A2.2: LC-ICPMS chromatograms of growth media extract from axenic Prochlorococcus AS9601 grown to late-log phase in media containing different iron concentrations. Abundance of the iron ligands scaled with culture fluorescence. 

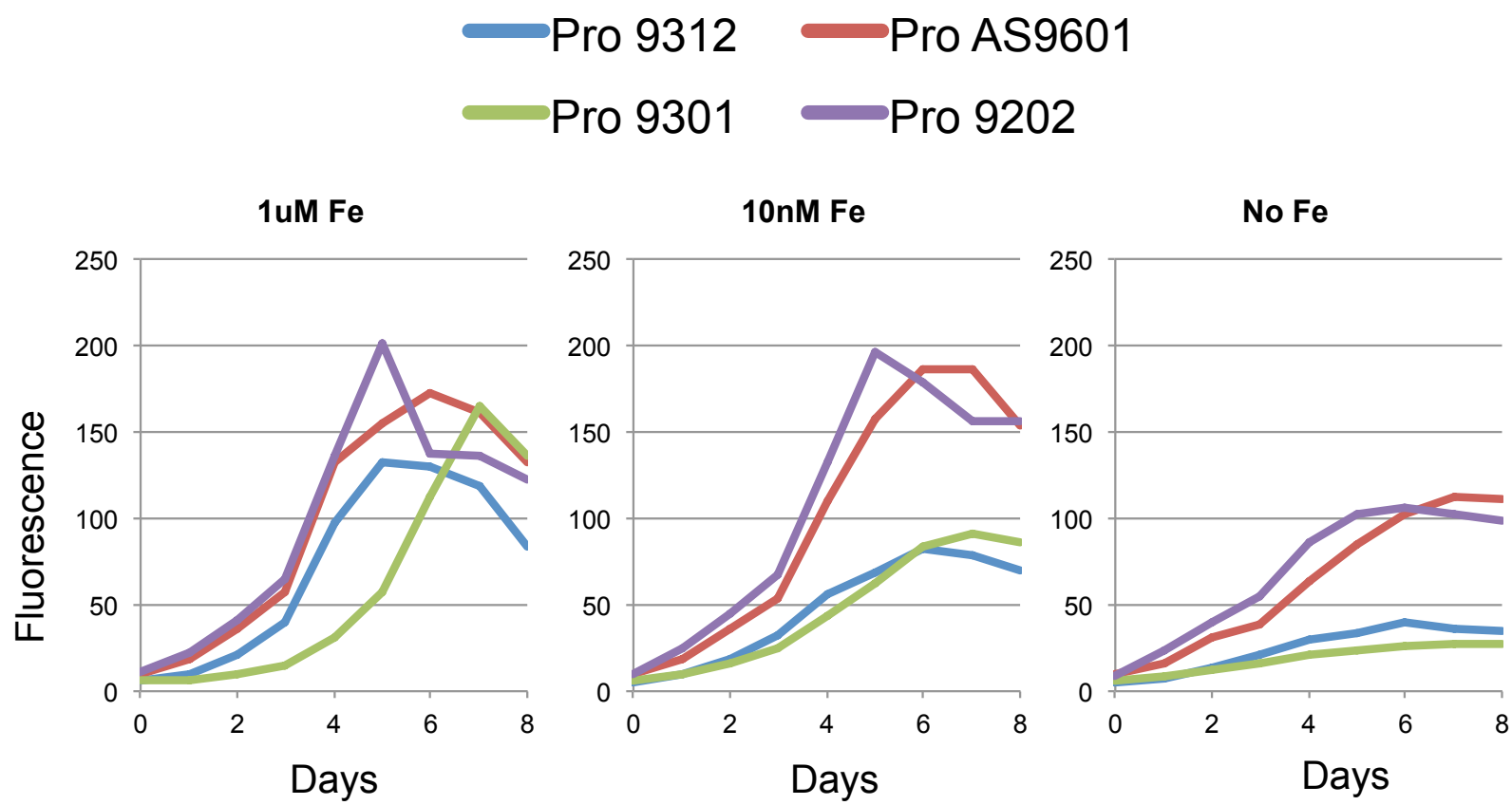

Figure A2.3: Growth curves for Prochlorococcus strains 9312, 9301, AS9601, and 9202. Lower added iron levels have a stronger negative effect on fluorescence levels of Prochlorococcus 9312 and 9301 compared with AS9601 and 9202, suggesting that the latter strains may be better adapted to low iron conditions. 


\title{
Chapter 3: An extended siderophore suite from Synechococcus sp. PCC 7002 revealed by LC-ICPMS-ESIMS.
}

Reproduced with permission from Metallomics, "Boiteau, R.M., Repeta, D.J., An extended siderophore suite from Synechococcus sp. PCC 7002 revealed by LC-ICPMS-ESIMS. Metallomics 7(5) 877-884." Copyright 2014 Royal Society of Chemistry.

\begin{abstract}
Siderophores are thought to play an important role in iron cycling in the ocean, but relatively few marine siderophores have been identified. Sensitive, high throughput methods hold promise for expediting the discovery and characterization of new siderophores produced by marine microbes. We developed a methodology for siderophore characterization that combines liquid chromatography (LC) inductively coupled plasma mass spectrometry (ICPMS) with high resolution electrospray ionization mass spectrometry (ESIMS). To demonstrate this approach, we investigated siderophore production by the marine cyanobacteria Synechococcus sp. PCC 7002. Three hydroxamate siderophores, synechobactin A-C, have been previously isolated and characterized from this strain. These compounds consist of an iron binding head group attached to a fatty acid side chain of variable length $\left(\mathrm{C}_{12}, \mathrm{C}_{10}\right.$, and $\mathrm{C}_{8}$ respectively). In this study, we detected six iron-containing compounds in Synechococcus sp. PCC 7002 media by LC-ICPMS. To identify the molecular ions of these siderophores, we aligned the chromatographic retention times of peaks from the LC-ICPMS chromatogram with features detected from LC-ESIMS spectra using an algorithm designed to recognize metal isotope patterns. Three of these compounds corresponded to synechobactins A $(614 \mathrm{~m} / \mathrm{z}), \mathrm{B}(586 \mathrm{~m} / \mathrm{z})$, and C $(558 \mathrm{~m} / \mathrm{z})$. The MS2 spectra of these compounds revealed diagnostic synechobactin fragmentation patterns which were used to confirm the identity of the three unknown compounds $(600,628$, and $642 \mathrm{~m} / \mathrm{z})$ as new members of the synechobactin suite with side chain lengths of 11, 13, and 14 carbons. These results demonstrate the potential of combined LCMS techniques for the identification of novel iron-organic complexes.
\end{abstract}




\subsection{Introduction:}

Iron availability can influence the biological productivity and ecosystem community composition of the ocean [Morel et al., 2003; De Baar et al., 2005; Moore and Doney, 2007; Sunda, 2012]. In oxic seawater, dissolved iron (III) rapidly forms insoluble oxyhydroxides and precipitates from solution [Liu and Millero, 2002]. This poses a challenge to microbes that require iron for photosynthesis, nitrogen fixation, respiration, protection against oxidative stress, and other essential biological processes. In regions where available iron is scarce, microbes may gain a competitive advantage by producing siderophores; biomolecules that strongly and specifically complex iron in a form that can be recovered using dedicated membrane transporters [Braun and Killmann, 1999; Wandersman and Delepelaire, 2004].

Isolating and identifying siderophores is important for understanding the competition for iron in regions of the ocean where iron scarcity exerts a selective pressure on the microbial community. Indeed, siderophores appear to be present at low concentrations in seawater collected from open ocean sites [Mawji et al., 2008b], and metagenomic analysis has suggested that siderophore production and uptake in the ocean may be common [Desai et al., 2012; Hopkinson and Barbeau, 2012; Toulza et al., 2012; Li et al., 2014]. Some siderophores have been discovered in the extracts of a number of marine bacteria grown in laboratory cultures [Butler, 2005]. However their characterization in marine environmental samples remains a challenge due to their low concentrations. Of the known marine siderophores, many are amphiphilic, consisting of a polar, iron-binding head group and a nonpolar fatty acid tail [Martinez et al., 2003; Martin et al., 2006; Martinez and Butler, 2007; Homann et al., 2009; Vraspir et al., 2011; Gauglitz and Butler, 2013], which may allow the siderophore to be tethered to the outer cell membrane or form micelles in an environment where free siderophores might otherwise diffuse away too quickly to facilitate iron uptake [Martinez et al., 2000; Xu et al., 2002]. It is common for amphiphilic siderophores to occur as homologous series with fatty acid tails that differ by $\mathrm{CH}_{2}$, such that a single bacteria may produce a suite of structurally related siderophores.

Liquid chromatography (LC) coupled to mass spectrometry offers a sensitive, high throughput means of detecting and characterizing siderophores. Two complementary methods have been 
used to detect organic-metal complexes: inductively coupled plasma mass spectrometry (ICPMS) and electrospray ionization mass spectrometry (ESIMS) [Lobiński et al., 2006; Mounicou et al., 2009; Pedrero et al., 2012; Grevenstuk et al., 2013; Grillet et al., 2014]. In the case of ICPMS, as the metal complexes elute from the chromatography column, the metal is atomized and detected directly as the elemental ion. This is a convenient method for sensitively detecting metal complexes and estimating their abundances in biological and environmental samples [Mawji et al., 2008b; Pröfrock and Prange, 2012; Boiteau et al., 2013; Clough et al., 2014; Lechtenfeld et al., 2014]. However, ICPMS provides no information on the structure of the organic complex. Electrospray ionization mass spectrometry (ESIMS) can be used to detect isotopologues of the intact metal complexes [Velasquez et al., 2011; Lehner et al., 2013; Baars et al., 2014; Deicke et al., 2014; Waska et al., 2015]. For metals such as iron that have multiple isotopes, computer algorithms can search LC-ESIMS mass spectra and identify features that match a metal's characteristic isotope pattern [Lehner et al., 2013; Baars et al., 2014; Deicke et al., 2014]. Furthermore, ESI instruments capable of generating MS2 spectra of the parent ion can reveal diagnostic fragmentation patterns that facilitate compound identification. However, it is difficult to quantify organic-metal complexes using ESIMS, and search algorithms often miss isotopologues that occur in low abundance or report false positives due to isobaric interferences from co-eluting compounds [Keith-Roach, 2010].

We developed a methodology that combines both LCMS techniques and used it to investigate the production of siderophores by Synechococcus sp. PCC 7002 (Fig. 1). The metabolites produced by this fast growing model marine cyanobacteria have been the focus of numerous studies [Baran et al., 2010, 2011, 2013; McNeely et al., 2010; Bennette et al., 2011; Lanekoff et al., 2013]. Synechococcus sp. PCC 7002 is known to produce a suite of three siderophores known as synechobactins A, B and C [Trick and Wilhelm, 1995; Wilhelm and Maxwell, 1996; Ito and Butler, 2005]. Synechobactins consist of a citrate head group attached to two 1,3diaminopropane moieties. The terminal amines of the diaminopropane moieties are hydroxylated and one is linked to acetic acid while the other is linked to a fatty acid (Fig. 2). Synechobactins A-C differ by the carbon number of their fatty acid chains, with synechobactin A having octanoic acid $\left(\mathrm{C}_{8}\right)$, $\mathrm{B}$ having decanoic acid $\left(\mathrm{C}_{10}\right)$, and $\mathrm{C}$ having dodecanoic acid $\left(\mathrm{C}_{12}\right)$ [Ito and Butler, 2005]. Additional iron complexes were later detected in the media of Synechococcus 
sp. PCC 7002 with LC-ICPMS [Boiteau et al., 2013]. Here we report the improved separation of Synechococcus sp. PCC 7002 siderophores and the use of an isotope matching algorithm to assign parent ion masses to three previously undescribed complexes. By comparing the MS2 fragmentation spectra of known synechobactins to the unknown complexes, we were able to identify them as new members of the synechobactin family of siderophores.

\subsection{Experimental:}

\subsubsection{Materials and reagents:}

Ultrahigh purity water $(18.2 \mathrm{M} \Omega \mathrm{cm})$, and LCMS grade methanol $(\mathrm{MeOH})$, ammonium formate, and formic acid (Optima, Fisher scientific) were used in this study. The methanol was further purified by sub-boiling-point distillation in a polytetrafluoroethylene (PTFE) still to reduce Fe contamination [Boiteau et al., 2013]. Nutrient salts and vitamins for culture media were obtained from Sigma Aldrich. Polycarbonate plastic bottles used for culturing and PTFE vials for sample storage were soaked overnight in $0.1 \%$ detergent (Citranox), rinsed $5 \mathrm{x}$ with $\mathrm{H}_{2} \mathrm{O}$, and then soaked in $1 \mathrm{~N}$ hydrochloric acid (J.T. Baker) for 2 days followed by a final $5 \mathrm{x}$ rinse with $\mathrm{H}_{2} \mathrm{O}$. PTFE and platinized silicone tubing (Cole Parmer) and tube adapters (Visiprep, Sigma Aldrich) used for solid phase extraction were cleaned by rinsing with $1 \mathrm{~N} \mathrm{HCl}$ through the tubing for 12 hrs followed by rinsing with $\mathrm{H}_{2} \mathrm{O}$ for another 12 hours using a peristaltic pump (Cole Parmer). All samples for LCMS analysis were placed in certified $2 \mathrm{~mL}$ amber glass autosampler vials or $250 \mu \mathrm{L}$ vial inserts (Agilent).

\subsubsection{Synechococcus sp. PCC 7002 culture:}

Cultures of Synechococcus sp. PCC 7002 were grown in polycarbonate bottles in continuous light at $23^{\circ} \mathrm{C}$. A $10 \mathrm{~mL}$ inoculum was used to inoculate $500 \mathrm{~mL}$ of sterile $\mathrm{SN}$ medium [Waterbury et al., 1986] containing only $50 \mathrm{nM} \mathrm{FeCl}{ }_{3} * 6 \mathrm{H}_{2} \mathrm{O}$ plus $1 \mu \mathrm{g} / \mathrm{L}$ cyanocobalamin. After seven days of growth, the culture media was centrifuged to remove cells, filtered $(0.2 \mu \mathrm{m}$ polyethersulfone sterivex, Millipore), and pumped through an ENV+ resin column $(1 \mathrm{~g}, 6 \mathrm{~mL}$, Biotage) at a flow rate of $10 \mathrm{~mL} / \mathrm{min}$. A $500 \mathrm{~mL}$ volume of sterile media was processed as a procedural blank to monitor contamination. The columns were rinsed with $\mathrm{H}_{2} \mathrm{O}$ to remove salts and eluted with $6 \mathrm{~mL}$ of distilled $\mathrm{MeOH}$ into PTFE vials. This organic extract was concentrated 
to $0.5 \mathrm{~mL}$ by evaporation under a stream of nitrogen gas and stored in the dark at $-20^{\circ} \mathrm{C}$ until analysis by LC-MS. Effort was made to minimize exposure of the sample to light and avoid photodegradation.

\subsubsection{Liquid chromatography:}

Organic extracts were separated using an Agilent 1260 series bioinert high pressure chromatography pump and autosampler fitted with a C18 column (Kinetex $2.1 \times 100 \mathrm{~mm}, 1.7 \mu \mathrm{m}$ particle size) and polyetheretherketone (PEEK) tubing and connectors. The mobile phase consisted of (A) $5 \mathrm{mM}$ aqueous ammonium formate or $0.1 \%$ formic acid in $\mathrm{H}_{2} \mathrm{O}$ (B) $5 \mathrm{mM}$ ammonium formate or $0.1 \%$ formic acid in distilled $\mathrm{MeOH}$. Optimal separation and electrospray ionization were achieved using ammonium formate buffer and a 30 minute solvent gradient from $5 \%$ to $100 \%$ B followed by a 10 minute isocratic elution in $100 \%$ B at a flow rate of $100 \mu \mathrm{L} / \mathrm{min}$. A post column PEEK flow splitter directed 50\% of the flow into the ICP-MS or ESI-MS. Reducing the flow entering the ICP-MS to $50 \mu \mathrm{L} / \mathrm{min}$ eliminated the need for post-column desolvation, even when eluting with $100 \%$ organic phase. On the same column, chromatographic retention times were reproducible within $0.1 \mathrm{~min}$ over several months of analysis.

\subsubsection{LC-ICPMS conditions:}

The flow of the LC column was coupled directly to a quadrupole ICPMS (Thermo ICAPq) using a teflon STD micronebulizer (ESI) and a cyclonic spray chamber cooled to $0^{\circ} \mathrm{C}$. Oxygen gas was introduced to the plasma at $25 \mathrm{~mL} / \mathrm{min}$ to combust organic solvents and buffers to $\mathrm{CO}_{2}$ thereby preventing the formation of reduced carbon deposits that would otherwise accumulate on the cones. The ICPMS was equipped with platinum sampler and skimmer cones, and was tuned each day using an automated tuning feature and the 'Tune B' solution purchased from Thermo

Scientific. ${ }^{54} \mathrm{Fe},{ }^{56} \mathrm{Fe},{ }^{57} \mathrm{Fe}$, and ${ }^{59} \mathrm{Co}$ were monitored with an integration time of 0.05 seconds each. The instrument was run in KED mode with a He collision gas introduced at a rate of 4.2 $\mathrm{mL} / \mathrm{min}$ to minimize $\mathrm{ArO}^{+}$interferences on ${ }^{56} \mathrm{Fe}$.

\subsubsection{LC-ESIMS conditions:}


For LC-ESIMS analysis, the flow from the LC was coupled to a Thermo Scientific Orbitrap Fusion mass spectrometer equipped with a heated electrospray ionization source. ESI source parameters were set to a capillary voltage of $3500 \mathrm{~V}$, sheath, auxiliary and sweep gas flow rates of 12,6 , and 2 (arbitrary units), and ion transfer tube and vaporizer temperatures of $300^{\circ} \mathrm{C}$ and $75^{\circ} \mathrm{C}$. MS1 scans were collected in high resolution $(450 \mathrm{~K})$ positive and negative mode. High energy collision induced dissociation (HCD) MS2 spectra for the most abundant compounds in each orbitrap scan were collected simultaneously on the ion trap mass analyzer. Ions were trapped using quadrupole isolation of $\mathrm{a} \pm 2 \mathrm{~m} / \mathrm{z}$ mass window and were then fragmented using an HCD collision energy of $35 \%$. For the six iron containing compounds investigated in this study, targeted high resolution MS2 spectra were collected with the orbitrap mass analyzer during a second analytical run to obtain accurate fragment masses.

\subsubsection{Data processing:}

The LC-ESIMS data was converted to mzXML file format (MSconvert, proteowizard) and iron isotopologue features were identified using a data-mining algorithm (script written in $\mathrm{R}$ using xcmsRaw class [Smith et al., 2006]). This algorithm searches through each ms scan and compiles a list of peak sets (retention time, masses, and intensities) that fit a specified isotope pattern. Both low tolerance and high tolerance (described below) peak ratio filters were used. The compiled peak sets are then binned by mass in $0.01 \mathrm{~m} / \mathrm{z}$ increments. Two criteria are used to automatically remove mass sets that result from instrumental noise rather than chromatographic peaks: (1) Mass sets that do not appear at least twice within a 10 second interval are discarded. (2) Mass sets that are found in 8 or more 30 second intervals are discarded if they do not contain any points above the intensity baseline. The baseline is calculated based on the maximum intensity points from each 30 second time interval. The highest $25 \%$ of intensities are discarded, and the baseline is calculated as $3 x$ the standard deviation plus the mean of the remaining values.

The resulting mass list was manually curated by inspecting the extracted ion chromatograms (EICs) of the putative isotopologues. A constant time offset was applied to the LC-ESIMS chromatogram in order to align the retention time of the cyanocobalamin $[\mathrm{M}+\mathrm{H}]^{2+}$ peak (EIC of $\mathrm{m} / \mathrm{z}=678$ ) with the cyanocobalamin ${ }^{59} \mathrm{Co}$ peak at $20.7 \mathrm{~min}$ in the LC-ICPMS chromatogram. Iron-containing ions were considered valid if peaks in the EICs: (1) Have the same retention time 
and peak shape compared to each other when the intensities are scaled relative to the expected isotope ratio. (2) Have the same retention time as the associated peak in the LC-ICPMS spectrum.

\subsection{Results and discussion}

\subsubsection{LC-ICP-MS:}

Tandem LC-ICPMS enables rapid detection of metal organic complexes. Over the course of the chromatographic separation, siderophores elute from the column and enter the plasma where the iron is atomized, ionized, and detected by the mass spectrometer as ${ }^{56} \mathrm{Fe}^{+}$. The chromatogram that results from this analysis indicates the retention time and abundance of each compound that contains iron.

LC-ICPMS was used to detect iron-binding compounds extracted from Synechoccocus sp. PCC 7002 culture (Fig. 3a). Chromatographic conditions were optimized to achieve baseline resolution of the compounds which appear as six distinct peaks of iron, with characteristic retention times of 29.4, 33.9, 35.8, 37.1, 38.5 and 39.6 minutes. This separation was achieved using a 30-minute gradient from $0-100 \%$ methanol using a $5 \mathrm{mM}$ aqueous ammonium formate buffer.

\subsubsection{LC-ESIMS:}

While ICPMS provides information on the quantity and number of different ligands in the samples along with their retention times, ESIMS provides complimentary information on the parent ion mass and fragmentation pattern. Using the same chromatography as described above, the Synechococcus media extract was analyzed by LC-ESIMS. As a soft ionization technique, ESIMS measures the mass of the intact metal-ligand complexes.

Samples were analyzed in both positive and negative ionization modes, with either $0.1 \%$ formic acid or $5 \mathrm{mM}$ ammonium formate as a mobile phase buffer. For the previously characterized siderophores (synechobactins $\mathrm{A}, \mathrm{B}$, and $\mathrm{C}$ ), the use of $5 \mathrm{mM}$ ammonium formate in positive 
mode resulted in the greatest parent ion signal intensity. The apo (metal-free) form of the siderophore was also detected under these conditions, although the intensity was $<2 \%$ relative to the iron-bound form. When $0.1 \%$ formic acid was used in the mobile phase, the parent ion signal decreased by a factor of 10 and the apo form intensity was $8-11 \%$ relative to the iron-bound form. While the intensity ratio between a ligand and its complex in ESI MS spectra does not necessarily reflect concentration ratio as the two species may ionize differently in an ESI source, these results demonstrate that ammonium formate is a preferred buffer for detecting iron-bound hydroxamate siderophores such as synechobactins.

To determine the masses of the three major unknown iron containing compounds, an $\mathrm{R}$ based algorithm was used to extract mass spectral features that matched the natural abundance pattern of iron stable isotopes. Results were manually curated to determine which matches aligned with one of the six iron peaks detected by LC-ICPMS (Fig. 3). The choice of isotope pattern criteria balances flexibility and specificity. Ideally, the selection criteria are flexible enough to account for imprecision in the instrumental measurement but specific enough to exclude other common isotope patterns and to reduce the risk of false positives that result from co-eluting ions or from instrumental noise that coincidentally match the specified isotope pattern.

The algorithm used in this study searched each spectral scan for pairs of peaks with a mass difference of $1.995 \mathrm{~m} / \mathrm{z}$ and a light/heavy intensity ratio of 0.06 , corresponding to isotopologues containing ${ }^{54} \mathrm{Fe}$ and ${ }^{56} \mathrm{Fe}$ respectively. A mass window of $\pm 3 \mathrm{mDa}$ was used to account for uncertainty in the accuracy of the orbitrap analyzer at 450,000 mass resolution. Since the uncertainty in the isotopologue intensity ratio depends on signal intensity, detecting low abundance isotope pairs requires a larger range in the intensity ratio criteria than is required for more abundant compounds. When a narrow ratio window $(0.06 \pm 0.015)$ was used, 40 isotope pairs were identified by the algorithm and 17 of these peaks passed the manual curation step. Using a wide ratio window $(0.06 \pm 0.036), 92$ isotope sets were initially identified, and 29 passed the manual curation step. The most abundant isotope pair found at each retention time was assigned as the parent ion (Fig. 3, Table 1). Other peak pairs corresponded to ${ }^{13} \mathrm{C}$ isotopologues as well as adducts with other abundant coeluting compounds (a list is included in the supplementary information, Fig. SI-1, Table SI-1). 


\subsubsection{MS2 Fragmentation:}

MS2 fragmentation patterns were used to structurally characterize the six synechobactins detected in this study (Fig. 4). Once the parent ion masses were assigned, the sample was reanalyzed by LC-ESIMS for targeted high resolution MS2 analysis. An isolation window of \pm 2 $\mathrm{m} / \mathrm{z}$ was used to retain the iron isotope patterns of fragments that contain iron.

First, the characteristic fragmentation patterns of synechobactins $A, B$, and $C(\mathrm{~m} / \mathrm{z} 614.261$, 586.23, and 558.198) were determined. Structurally, synechobactins consists of an iron binding head group composed of citrate and two aminopropane moieties linked to a fatty acid or acetic acid. Most of the major fragments that were observed for the known synechobactins retain iron (based on the appearance of ions corresponding to the ${ }^{54} \mathrm{Fe}$ and ${ }^{56} \mathrm{Fe}$ isotopologues). The MS2 analysis of all three synechobactins displayed a major fragment with neutral losses of 156.006 [$\mathrm{C}_{6} \mathrm{H}_{4} \mathrm{O}_{5}$ ], corresponding to cleavage at the amide linkage and loss of citrate (Fig. 5a). This fragmentation pattern is consistent with those of other citrate and hydroxamate based siderophores [Gledhill, 2001; Mawji et al., 2008a; Gauglitz et al., 2012]. Other major neutral mass losses correspond to fragmentation within the citrate head group including 46.006 [$\left.\mathrm{CH}_{2} \mathrm{O}_{2}\right], 113.996\left[-\mathrm{C}_{4} \mathrm{H}_{2} \mathrm{O}_{4}\right]$, and 130.027 [ $\left.-\mathrm{C}_{5} \mathrm{H}_{6} \mathrm{O}_{4}\right]$. Several additional fragments indicate head group cleavages that result in the loss of one of the hydroxamate groups (Fig. 5b). For synechobactin $A$, for instance, these fragments occur in pairs that differ by $\mathrm{C}_{10} \mathrm{H}_{20}(140.157 \mathrm{~m} / \mathrm{z})$, corresponding to the loss of the long fatty acid hydroxamate side chain (including fragments at 206.035, 270.030, 288.040, 298.025, Fig. 4d) and the acetohydroxamate respectively (fragments at 346.191, 410.186, 428.198, and 438.181, Fig. 4d). MS2 spectra of the positive apo (iron free) ion of synechobactin A-B and the negative iron-bound ion of synechobactin A were also measured (supplementary information, Fig. SI-2).

The fragmentation patterns of synechobactins A-C help in the interpretation of MS2 spectra of the three uncharacterized siderophores. The mass difference between the unknown compounds and synechobactins A-C correspond to the addition or loss of $\left(\mathrm{CH}_{2}\right)_{\mathrm{n}}(\Delta \mathrm{m} / \mathrm{z}=\mathrm{n} * 14)$. All three uncharacterized siderophores exhibit neutral losses that are characteristic of fragmentation of a 
citrate head group including $46.006\left[-\mathrm{CH}_{2} \mathrm{O}_{2}\right], 113.996\left[-\mathrm{C}_{4} \mathrm{H}_{2} \mathrm{O}_{4}\right], 130.027\left[-\mathrm{C}_{5} \mathrm{H}_{6} \mathrm{O}_{4}\right]$, and $156.006\left[-\mathrm{C}_{6} \mathrm{H}_{4} \mathrm{O}_{5}\right]$. They also contain the 206.035, 270.030, 288.040, and 298.025 fragments that are attributed to cleavage across the citrate head group that retains the acetohydroxamate group. Thus, these new compounds vary by the length of the fatty acid side chain, and correspond to the $\mathrm{C}_{13}, \mathrm{C}_{15}$, and $\mathrm{C}_{16}$ synechobactins.

The MS2 spectra of the low abundance siderophores with $\mathrm{m} / \mathrm{z}$ of 600.245 and 628.276 contain additional fragments from coeluting compounds (red masses, Fig. 4). By obtaining off-peak MS2 scans from the interfering parent ions, we were able to identify the masses that arise from the interfering ion rather than the siderophore (supplementary information, Fig. SI-3).

\subsubsection{Connection to synechobactin biosynthesis:}

The six major compounds detected by LC-ICPMS were all identified as homologues of synechobactin A. A search for ions in the LC-ESIMS spectra that correspond to other synechobactin homologues revealed low intensity peaks that potentially correspond to $\mathrm{C}_{9}$ synechobactin and $\mathrm{C}_{16}$-synechobactin, although the ${ }^{54} \mathrm{Fe}$ isotopologues for both were below detection (supplementary information, Fig. SI-4). These results reveal that Synechococcus sp. PCC 7002 is capable of incorporating a wide range of fatty acids into the hydroxamate side chain of synechobactin. There appears to be a preference for an even number of carbons over odd carbon numbers. Additional synechobactins with longer hydrophobic side chains may have been present in the sample, but were not recovered from the extraction column by the methanol wash.

Such a wide range of siderophores produced by Synechococcus sp. PCC 7002 suggests substrate flexibility for the enzymes involved in synechobactin biosynthesis. The operon responsible for synechobactin synthesis (SYNPCC7002_G0019-G0024) is located on a plasmid that is largely dedicated to the synthesis, uptake, and processing of these compounds, and these genes are upregulated in low iron conditions [Ludwig and Bryant, 2012]. Synechobactin synthesis appears to be similar to the synthesis of rhizobactin, which is structurally equivalent to synechobactin $B$ $\left(\mathrm{C}_{10}\right)$ with a trans double bond between carbons 2,3 of the fatty acid side chain (Fig. 2). The production of rhizobactin is thought to involve the synthesis of schizokinen, which contains two 
acetohydroxamates [Mullis et al., 1971], followed by the replacement of one acetic acid with 2decenoic acid [Lynch et al., 2001]. It also is possible that Synecoccoccus sp. PCC 7002 uses schizokinen as an intermediate in synechobactin synthesis. Indeed, in a culture of Synechococcus sp. PCC 7002 that was harvested at an earlier growth phase, an additional chromatographic ${ }^{56} \mathrm{Fe}$ peak was observed [Boiteau et al., 2013] with $\mathrm{m} / \mathrm{z} 474$ that corresponds to schizokinen (supplementary information Fig. SI-5). A putative lipase that is encoded at the end of the synechobactin biosynthesis operon (SYNPCC7002_G0018) may be responsible for nonspecifically catalyzing the attachment of the fatty acid side chain to the synechobactin precursor. A better understanding of the metabolic pathways involved in catalyzing this reaction will help reveal how amphiphilic marine siderophores are produced, shed light on how this functionality evolved, and will improve our ability to search for amphiphilic siderophore biosynthetic potential in marine genomic/metagenomic libraries.

\subsection{Conclusions}

The methodology described here provides a means to confidently detect and determine the masses and MS2 spectra of even minor siderophores in a biological extract with no prior knowledge of the siderophores produced in a culture. Common siderophore functional groups can have diagnostic fragmentation patterns that facilitate structural characterization of new siderophores, as illustrated in this study with the citrate head group fragmentation of the synechobactins. Using this approach, we were able to characterize three new synechobactins, and provide preliminary evidence for two additional homologues that may be present at very low concentrations. As MS2 libraries of known siderophores grow, our ability to characterize new siderophores based on high resolution mass spectrometry analysis will improve.

The combination of LC-ICPMS and LC-ESIMS has the potential to increase the pace and depth of siderophore discovery. Looking ahead, this work represents a step towards the eventual goal of detecting and characterizing metal-binding organic compounds directly in environmental samples where they impact ecosystems and trace metal cycling. Organic complexation is thought to largely control iron solubility and bioavailability in the ocean [Hunter and Boyd, 2007; Gledhill and Buck, 2012; Kraemer et al., 2014], and understanding the speciation of iron is 
critical for forecasting ecosystem changes in low iron regions of the ocean [Tagliabue et al., 2009; Shi et al., 2010; Tagliabue and Völker, 2011]. The challenge of characterizing these marine iron binding molecules (including siderophores [Mawji et al., 2008b, 2011], heme [Gledhill, 2014; Hogle et al., 2014], polysaccharides [Hassler et al., 2011, 2014], and humic substances [Laglera and van den Berg, 2009; Yang and Van den Berg, 2009; Batchelli et al., 2010; Laglera et al., 2011; Misumi et al., 2013; Bundy et al., 2015]) lies in their low concentrations (often sub-picomolar for a specific compound) within a very complex organic matrix. Methods such as the one described in this study have the potential to address these challenges and reveal the structural diversity of metal organic ligands in environmental samples.

\subsection{Acknowledgements}

We would like to thank Matt McIlvin for his assistance with the orbitrap mass spectrometer, John Waterbury and Athena Archer for supplying the Synechococcus sp. PCC 7002 strain, and two anonymous reviewers for their constructive comments. This work was supported by the

National Science Foundation program in Chemical Oceanography (OCE-1356747), and by the National Science Foundation Science and Technology Center for Microbial Oceanography Research and Education (C-MORE; DBI-0424599).

\subsection{References}

De Baar, H., P. Boyd, K. Coale, and M. Landry (2005), Synthesis of iron fertilization experiments: From the Iron Age in the Age of Enlightenment, J. Geophys. Res, 110(C9), 124, doi:10.1029/2004JC002601.

Baars, O., F. M. M. Morel, and D. H. Perlman (2014), ChelomEx: Isotope-Assisted Discovery of Metal Chelates in Complex Media Using High-Resolution LC-MS, Anal. Chem., 86(22), 11298-11305, doi:10.1021/ac503000e.

Baran, R., B. P. Bowen, N. J. Bouskill, E. L. Brodie, S. M. Yannone, and T. R. Northen (2010), Metabolite Identification in Synechococcus sp. PCC 7002 Using Untargeted Stable Isotope Assisted Metabolite Profiling., Anal. Chem., 82(21), 9034-9042, doi:10.1021/ac1020112.

Baran, R., B. P. Bowen, and T. R. Northen (2011), Untargeted metabolic footprinting reveals a surprising breadth of metabolite uptake and release by Synechococcus sp. PCC 7002., Mol. Biosyst., 7(12), 3200-6, doi:10.1039/c1mb05196b. 
Baran, R., N. N. Ivanova, N. Jose, F. Garcia-Pichel, N. C. Kyrpides, M. Gugger, and T. R. Northen (2013), Functional genomics of novel secondary metabolites from diverse cyanobacteria using untargeted metabolomics., Mar. Drugs, 11(10), 3617-31, doi:10.3390/md11103617.

Batchelli, S., F. L. L. Muller, K.-C. Chang, and C. Lee (2010), Evidence for strong but dynamic iron-humic colloidal associations in humic-rich coastal waters., Environ. Sci. Technol., 44(22), 8485-90, doi:10.1021/es101081c.

Bennette, N., J. Eng, and G. Dismukes (2011), An LC-MS-based chemical and analytical method for targeted metabolite quantification in the model cyanobacterium Synechococcus sp. PCC 7002, Anal. Chem., 83(10), 3808-16, doi:10.1021/ac200108a.

Boiteau, R. M., J. N. Fitzsimmons, D. J. Repeta, and E. A. Boyle (2013), Detection of iron ligands in seawater and marine cyanobacteria cultures by high-performance liquid chromatography-inductively coupled plasma-mass spectrometry., Anal. Chem., 85(9), 4357-62, doi:10.1021/ac3034568.

Braun, V., and H. Killmann (1999), Bacterial solutions to the iron-supply problem., Trends Biochem. Sci., 24(3), 104-9.

Bundy, R. M., H. A. N. Abdulla, P. G. Hatcher, D. V. Biller, K. N. Buck, and K. A. Barbeau (2015), Iron-binding ligands and humic substances in the San Francisco Bay estuary and estuarine-influenced shelf regions of coastal California, Mar. Chem., 173, 183-194, doi:10.1016/j.marchem.2014.11.005.

Butler, A. (2005), Marine siderophores and microbial iron mobilization, Biometals, 18(4), 369374, doi:10.1007/s10534-005-3711-0.

Clough, R., C. F. Harrington, S. J. Hill, Y. Madrid, and J. F. Tyson (2014), Atomic spectrometry updates. Review of advances in elemental speciation, J. Anal. At. Spectrom., 29(7), 1158, doi:10.1039/c4ja90029d.

Deicke, M., J. F. Mohr, J.-P. Bellenger, and T. Wichard (2014), Metallophore mapping in complex matrices by metal isotope coded profiling of organic ligands, Analyst, 139, 60966099, doi:10.1039/C4AN01461H.

Desai, D. K., F. D. Desai, and J. La Roche (2012), Factors influencing the diversity of iron uptake systems in aquatic microorganisms., Front. Microbiol., 3(October), 362, doi:10.3389/fmicb.2012.00362.

Gauglitz, J. M., and A. Butler (2013), Amino acid variability in the peptide composition of a suite of amphiphilic peptide siderophores from an open ocean Vibrio species., J. Biol. Inorg. Chem., 18(5), 489-97, doi:10.1007/s00775-013-0995-3. 
Gauglitz, J. M., H. Zhou, and A. Butler (2012), A suite of citrate-derived siderophores from a marine Vibrio species isolated following the Deepwater Horizon oil spill., J. Inorg. Biochem., 107(1), 90-5, doi:10.1016/j.jinorgbio.2011.10.013.

Gledhill, M. (2001), Electrospray ionisation-mass spectrometry of hydroxamate siderophores, Analyst, 126(8), 1359-1362, doi:10.1039/b1012681.

Gledhill, M. (2014), The detection of iron protoporphyrin (heme b) in phytoplankton and marine particulate material by electrospray ionisation mass spectrometry - comparison with diode array detection., Anal. Chim. Acta, 841,33-43, doi:10.1016/j.aca.2014.06.045.

Gledhill, M., and K. N. Buck (2012), The organic complexation of iron in the marine environment: a review., Front. Microbiol., 3, 1-17, doi:10.3389/fmicb.2012.00069.

Grevenstuk, T., P. Flis, L. Ouerdane, R. Lobinski, and A. Romano (2013), Identification of the tri-Al tricitrate complex in Plantago almogravensis by hydrophilic interaction LC with parallel ICP-MS and electrospray Orbitrap MS/MS detection., Metallomics, 5(9), 1285-93, doi:10.1039/c3mt00101f.

Grillet, L., L. Ouerdane, P. Flis, M. T. T. Hoang, M. Isaure, R. Lobinski, C. Curie, and S. Mari (2014), Ascorbate efflux as a new strategy for iron reduction and transport in plants., $J$. Biol. Chem., 289(5), 2515-25, doi:10.1074/jbc.M113.514828.

Hassler, C. S., V. Schoemann, C. M. Nichols, E. C. V. Butler, and P. W. Boyd (2011), Saccharides enhance iron bioavailability to Southern Ocean phytoplankton, Proc. Natl. Acad. Sci., 108(3), 1076-1081, doi:10.1073/pnas.1010963108.

Hassler, C. S., L. Norman, C. A. Mancuso Nichols, L. A. Clementson, C. Robinson, V. Schoemann, R. J. Watson, and M. A. Doblin (2014), Iron associated with exopolymeric substances is highly bioavailable to oceanic phytoplankton, Mar. Chem., doi:10.1016/j.marchem.2014.10.002.

Hogle, S. L., K. A. Barbeau, and M. Gledhill (2014), Heme in the marine environment: from cells to the iron cycle., Metallomics, 6(6), 1107-20, doi:10.1039/c4mt00031e.

Homann, V. V, M. Sandy, J. A. Tincu, A. S. Templeton, B. M. Tebo, and A. Butler (2009), Loihichelins A-F, a suite of amphiphilic siderophores produced by the marine bacterium Halomonas LOB-5., J. Nat. Prod., 72(5), 884-8, doi:10.1021/np800640h.

Hopkinson, B. M., and K. A. Barbeau (2012), Iron transporters in marine prokaryotic genomes and metagenomes., Environ. Microbiol., 14(1), 114-28, doi:10.1111/j.14622920.2011.02539.x.

Hunter, K. A., and P. W. Boyd (2007), Iron-binding ligands and their role in the ocean biogeochemistry of iron, Environ. Chem., 4(4), 221, doi:10.1071/EN07012. 
Ito, Y., and A. Butler (2005), Structure of synechobactins, new siderophores of the marine cyanobacterium Synechococcus sp. PCC 7002, Limnol. Oceanogr., 50(6), 1918-1923, doi:10.4319/1o.2005.50.6.1918.

Keith-Roach, M. J. (2010), A review of recent trends in electrospray ionisation-mass spectrometry for the analysis of metal-organic ligand complexes., Anal. Chim. Acta, 678(2), 140-8, doi:10.1016/j.aca.2010.08.023.

Kraemer, S. M., O. W. Duckworth, J. M. Harrington, and W. D. C. Schenkeveld (2014), Metallophores and Trace Metal Biogeochemistry, in Aquatic Geochemistry.

Krey, W. B. (2008), Siderophore Production by Heterotrophic Bacterial Isolates from the Costa Rica Upwelling Dome, Massachusetts Institute of Technology.

Laglera, L. M., and C. M. G. van den Berg (2009), Evidence for geochemical control of iron by humic substances in seawater, Limnol. Oceanogr., 54(2), 610-619.

Laglera, L. M., G. Battaglia, and C. M. G. van den Berg (2011), Effect of humic substances on the iron speciation in natural waters by CLE/CSV, Mar. Chem., 127(1-4), 134-143, doi:10.1016/j.marchem.2011.09.003.

Lanekoff, I., O. Geydebrekht, G. E. Pinchuk, A. E. Konopka, and J. Laskin (2013), Spatially resolved analysis of glycolipids and metabolites in living Synechococcus sp. PCC 7002 using nanospray desorption electrospray ionization., Analyst, 138(7), 1971-8, doi:10.1039/c3an36716a.

Lechtenfeld, O. J., G. Kattner, R. Flerus, S. L. McCallister, P. Schmitt-Kopplin, and B. P. Koch (2014), Molecular transformation and degradation of refractory dissolved organic matter in the Atlantic and Southern Ocean, Geochim. Cosmochim. Acta, 126, 321-337, doi:10.1016/j.gca.2013.11.009.

Lehner, S. M., L. Atanasova, N. K. N. Neumann, R. Krska, M. Lemmens, I. S. Druzhinina, and R. Schuhmacher (2013), Isotope-assisted screening for iron-containing metabolites reveals a high degree of diversity among known and unknown siderophores produced by Trichoderma spp., Appl. Environ. Microbiol., 79(1), 18-31, doi:10.1128/AEM.02339-12.

Li, M., B. M. Toner, B. J. Baker, J. A. Breier, C. S. Sheik, and G. J. Dick (2014), Microbial iron uptake as a mechanism for dispersing iron from deep-sea hydrothermal vents., Nat. Commun., 5, 3192, doi:10.1038/ncomms4192.

Liu, X., and F. Millero (2002), The solubility of iron in seawater, Mar. Chem., 77, 43-54.

Lobiński, R., D. Schaumlöffel, and J. Szpunar (2006), Mass spectrometry in bioinorganic analytical chemistry., Mass Spectrom. Rev., 25(2), 255-89, doi:10.1002/mas.20069.

Ludwig, M., and D. A. Bryant (2012), Acclimation of the Global Transcriptome of the 
Cyanobacterium Synechococcus sp. Strain PCC 7002 to Nutrient Limitations and Different Nitrogen Sources., Front. Microbiol., 3(April), 145, doi:10.3389/fmicb.2012.00145.

Lynch, D., J. O’Brien, T. Welch, P. Clarke, P. O. Cuív, J. H. Crosa, and M. O’Connell (2001), Genetic organization of the region encoding regulation, biosynthesis, and transport of rhizobactin 1021, a siderophore produced by Sinorhizobium meliloti., J. Bacteriol., 183(8), 2576-85, doi:10.1128/JB.183.8.2576-2585.2001.

Martin, J. D., Y. Ito, V. V Homann, M. G. Haygood, and A. Butler (2006), Structure and membrane affinity of new amphiphilic siderophores produced by Ochrobactrum sp. SP18., J. Biol. Inorg. Chem., 11(5), 633-41, doi:10.1007/s00775-006-0112-y.

Martinez, J., G. Zhang, P. Holt, and H. Jung (2000), Self-assembling amphiphilic siderophores from marine bacteria, Science, 287(5456), 1245-1247, doi:10.1126/science.287.5456.1245.

Martinez, J. S., and A. Butler (2007), Marine amphiphilic siderophores: marinobactin structure, uptake, and microbial partitioning, J. Inorg. Biochem., 101(805), 1692-1698, doi:10.1016/j.jinorgbio.2007.07.007.Marine.

Martinez, J. S., J. N. Carter-Franklin, E. L. Mann, J. D. Martin, M. G. Haygood, and A. Butler (2003), Structure and membrane affinity of a suite of amphiphilic siderophores produced by a marine bacterium., Proc. Natl. Acad. Sci. U. S. A., 100(7), 3754-9, doi:10.1073/pnas.0637444100.

Mawji, E., M. Gledhill, P. J. Worsfold, and E. P. Achterberg (2008a), Collision-induced dissociation of three groups of hydroxamate siderophores : ferrioxamines, ferrichromes and coprogens / fusigens, Rapid Commun. Mass Spectrom., (22), 2195-2202, doi:10.1002/rcm.

Mawji, E., M. Gledhill, J. A. Milton, G. A. Tarran, S. Ussher, A. Thompson, G. A. Wolff, P. J. Worsfold, and E. P. Achterberg (2008b), Hydroxamate siderophores: occurrence and importance in the Atlantic Ocean., Environ. Sci. Technol., 42(23), 8675-80.

Mawji, E., M. Gledhill, J. A. Milton, M. V. Zubkov, A. Thompson, G. A. Wolff, and E. P. Achterberg (2011), Production of siderophore type chelates in Atlantic Ocean waters enriched with different carbon and nitrogen sources, Mar. Chem., 124(1-4), 90-99, doi:10.1016/j.marchem.2010.12.005.

McNeely, K., Y. Xu, N. Bennette, D. a Bryant, and G. C. Dismukes (2010), Redirecting reductant flux into hydrogen production via metabolic engineering of fermentative carbon metabolism in a cyanobacterium., Appl. Environ. Microbiol., 76(15), 5032-8, doi:10.1128/AEM.00862-10.

Misumi, K., K. Lindsay, J. K. Moore, S. C. Doney, D. Tsumune, and Y. Yoshida (2013), Humic substances may control dissolved iron distributions in the global ocean: Implications from numerical simulations, Global Biogeochem. Cycles, 27(2), 450-462, 
doi:10.1002/gbc.20039.

Moore, J. K., and S. C. Doney (2007), Iron availability limits the ocean nitrogen inventory stabilizing feedbacks between marine denitrification and nitrogen fixation, Global Biogeochem. Cycles, 21(2), doi:10.1029/2006GB002762.

Morel, F. M. M., A. J. Milligan, and M. A. Saito (2003), Marine Bioinorganic Chemistry: The Role of Trace Metals in the Oceanic Cycles of Major Nutrients, Treatise Geochemistry, Vol. 6.

Mounicou, S., J. Szpunar, and R. Lobinski (2009), Metallomics: the concept and methodology., Chem. Soc. Rev., 38(4), 1119-38, doi:10.1039/b713633c.

Mullis, K. B., J. R. Pollack, and J. B. Neilands (1971), Structure of Schizokinen, an IronTransport Compound from Bacillus megaterium, Biochemistry, 10(26), 4894-4898.

Neilands, J. B., and K. Nakamura (1991), Detection, Determination, Isolation, Characterization and Regulation of Microbial Iron Chelates, in CRC Handbook of Microbial Iron Chelates, edited by G. Winkelmann, pp. 1-14, CRC Press, Boca Raton, FL.

Pedrero, Z., L. Ouerdane, S. Mounicou, R. Lobinski, M. Monperrus, and D. Amouroux (2012), Identification of mercury and other metals complexes with metallothioneins in dolphin liver by hydrophilic interaction liquid chromatography with the parallel detection by ICP MS and electrospray hybrid linear/orbital trap MS/MS., Metallomics, 4(5), 473-9, doi:10.1039/c2mt00006g.

Pröfrock, D., and A. Prange (2012), Inductively coupled plasma-mass spectrometry (ICP-MS) for quantitative analysis in environmental and life sciences: a review of challenges, solutions, and trends., Appl. Spectrosc., 66(8), 843-68, doi:10.1366/12-06681.

Saito, M. A., G. Rocap, and J. W. Moffett (2005), Production of cobalt binding ligands in a Synechococcus feature at the Costa Rica upwelling dome, Limnol. Oceanogr., 50(1), 279290, doi:10.4319/10.2005.50.1.0279.

Schwyn, B., and J. B. Neilands (1987), Universal chemical assay for the detection and determination of siderophores., Anal. Biochem., 160(1), 47-56.

Shi, D., Y. Xu, B. M. Hopkinson, and F. M. M. Morel (2010), Effect of ocean acidification on iron availability to marine phytoplankton., Science, 327(5966), 676-9, doi:10.1126/science.1183517.

Smith, C. A., E. J. Want, G. O’Maille, R. Abagyan, and G. Siuzdak (2006), XCMS: processing mass spectrometry data for metabolite profiling using nonlinear peak alignment, matching, and identification., Anal. Chem., 78(3), 779-87, doi:10.1021/ac051437y.

Sunda, W. G. (2012), Feedback Interactions between Trace Metal Nutrients and Phytoplankton 
in the Ocean., Front. Microbiol., 3(June), 204, doi:10.3389/fmicb.2012.00204.

Tagliabue, A., and C. Völker (2011), Towards accounting for dissolved iron speciation in global ocean models, Biogeosciences, 8(10), 3025-3039, doi:10.5194/bg-8-3025-2011.

Tagliabue, A., L. Bopp, O. Aumont, and K. R. Arrigo (2009), Influence of light and temperature on the marine iron cycle: From theoretical to global modeling, Global Biogeochem. Cycles, 23(2), doi:10.1029/2008GB003214.

Toulza, E., A. Tagliabue, S. Blain, and G. Piganeau (2012), Analysis of the global ocean sampling (GOS) project for trends in iron uptake by surface ocean microbes., PLoS One, 7(2), e30931, doi:10.1371/journal.pone.0030931.

Trick, C., and S. Wilhelm (1995), Physiological changes in the coastal marine cyanobacterium Synechococcus sp. PCC 7002 exposed to low ferric ion levels, Mar. Chem., 50(1-4), 207217, doi:10.1016/0304-4203(95)00036-Q.

Velasquez, I., B. L. Nunn, E. Ibisanmi, D. R. Goodlett, K. A. Hunter, and S. G. Sander (2011), Detection of hydroxamate siderophores in coastal and Sub-Antarctic waters off the South Eastern Coast of New Zealand, Mar. Chem., 126(1-4), 97-107, doi:10.1016/j.marchem.2011.04.003.

Vraspir, J. M., P. D. Holt, and A. Butler (2011), Identification of new members within suites of amphiphilic marine siderophores., Biometals, 24(1), 85-92, doi:10.1007/s10534-010-93781.

Wandersman, C., and P. Delepelaire (2004), Bacterial iron sources: from siderophores to hemophores., Annu. Rev. Microbiol., 58, 611-47, doi:10.1146/annurev.micro.58.030603.123811.

Waska, H., A. Koschinsky, M. J. Ruiz Chancho, and T. Dittmar (2015), Investigating the potential of solid-phase extraction and Fourier-transform ion cyclotron resonance mass spectrometry (FT-ICR-MS) for the isolation and identification of dissolved metal-organic complexes from natural waters, Mar. Chem., 173, 78-92, doi:10.1016/j.marchem.2014.10.001.

Waterbury, J., S. Watson, F. Valois, and D. Franks (1986), Biological and ecological characterization of the marine unicellular cyanobacterium Synechococcus, Can Bull Fish Aquat Sci, 214, 71.

Wilhelm, S., and D. Maxwell (1996), Growth, iron requirements, and siderophore production in iron-limited Synechococcus PCC 7002, Limnol. Oceanogr., 41(1), 89-97.

Xu, G., J. S. Martinez, J. T. Groves, and A. Butler (2002), Membrane affinity of the amphiphilic marinobactin siderophores., J. Am. Chem. Soc., 124(45), 13408-15. 
Yang, R., and C. M. G. Van den Berg (2009), Metal complexation by humic substances in seawater., Environ. Sci. Technol., 43(19), 7192-7. 


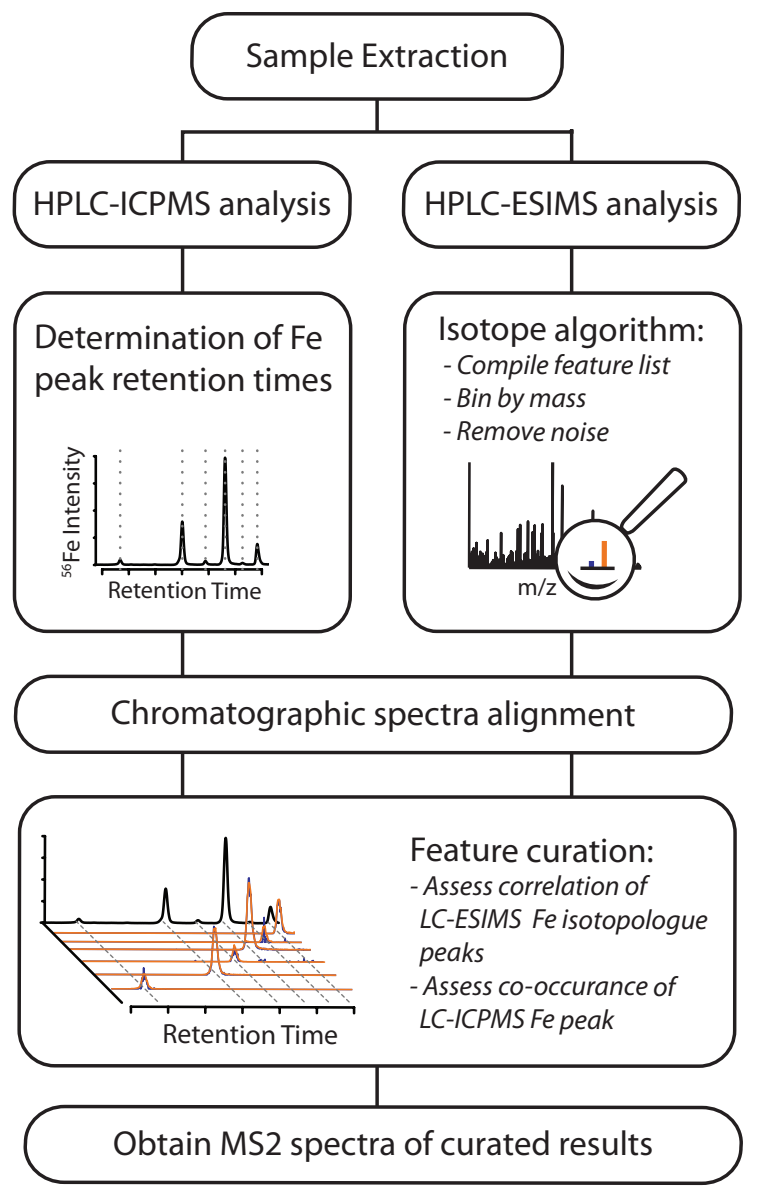

Figure 3.1: Workflow for unknown iron compound identification by combined LC-ICPMSESIMS. 


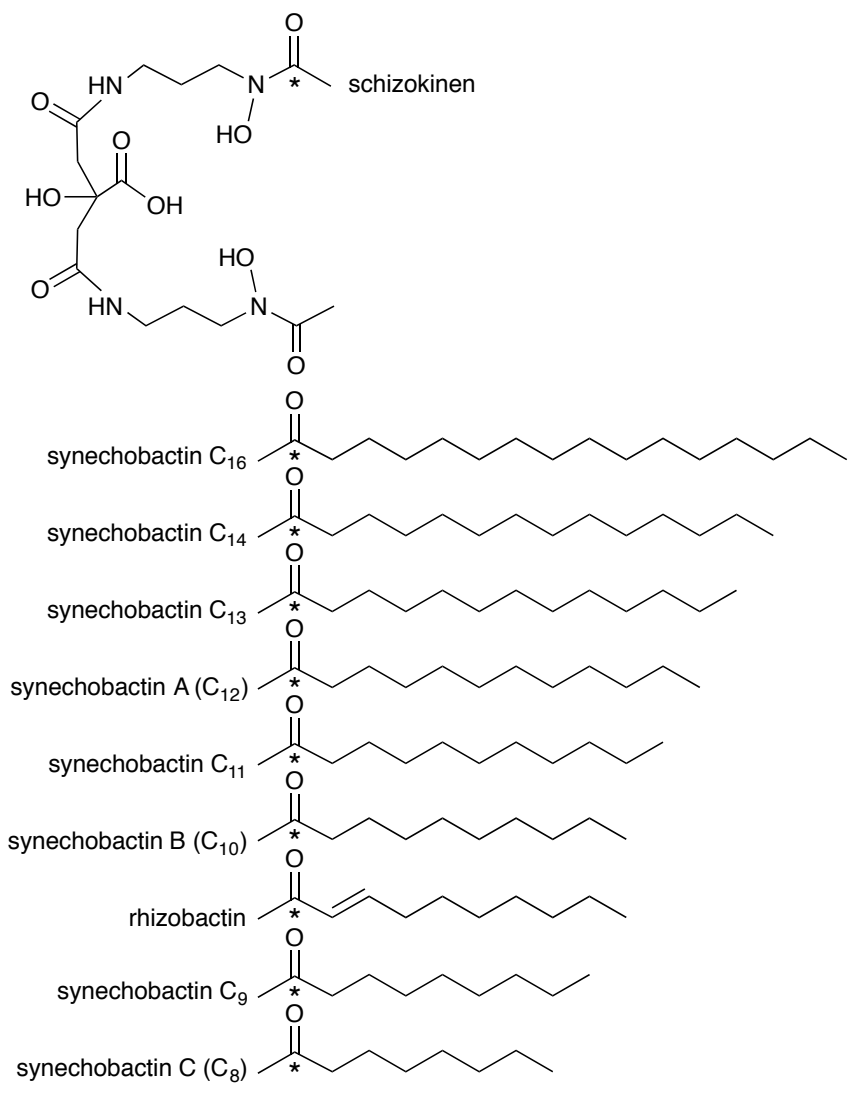

Figure 3.2: Chemical structure of the synechobactins and related compounds. The hydroxamate side chain (indicated by *) differentiates these compounds. 


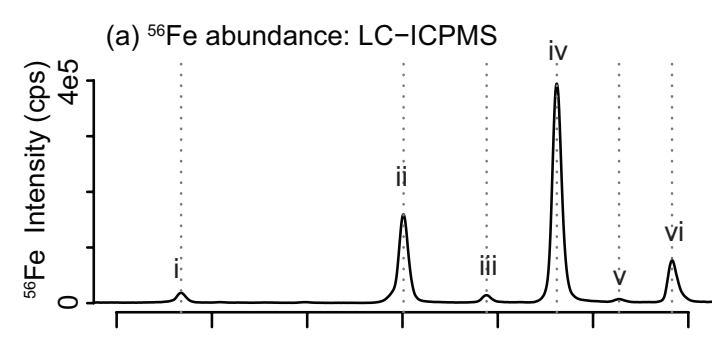

(b) Fe Isotopologues: LC-ESIMS

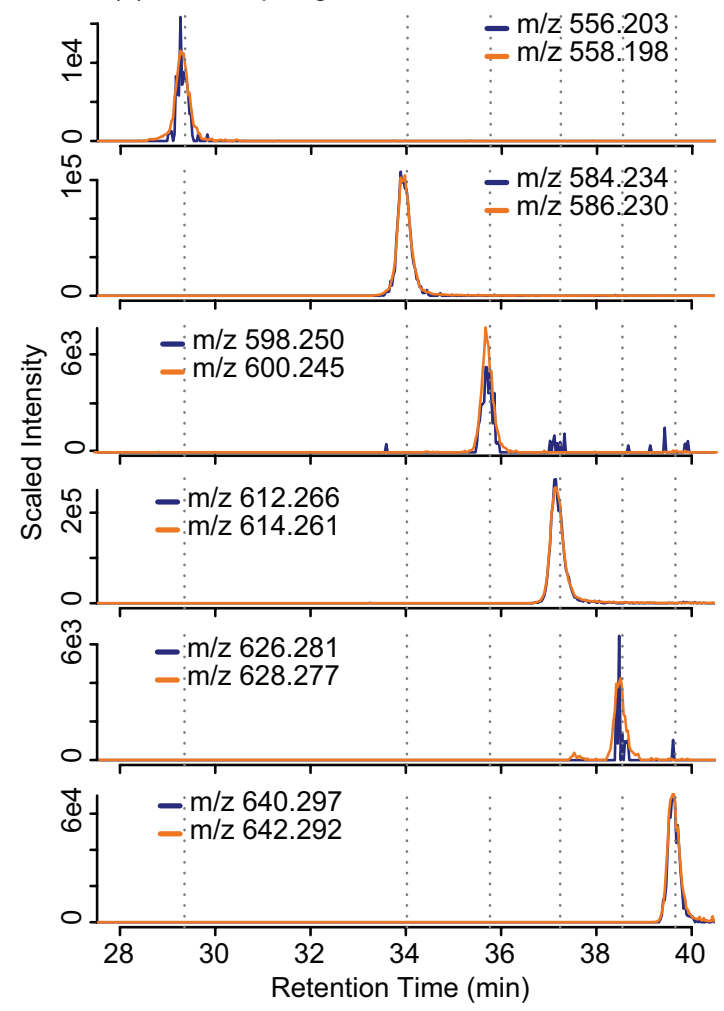

Figure 3.3: LC-MS chromatograms of Synechoccoccus sp. PCC 7002 media extract. (a) ${ }^{56} \mathrm{Fe}$ LC-ICPMS chromatogram. The six labeled peaks correspond to siderophores produced by Synechococcus sp. PCC 7002 in this experiment (b) extracted ion chromatograms from positive mode LC-ESIMS runs. Blue lines correspond to the light iron isotopologue $\left[\mathrm{M}+{ }^{54} \mathrm{Fe}^{3+}-2 \mathrm{H}^{+}\right]$that were identified by the isotope algorithm, and orange lines correspond to the heavy iron isotopologue $\left[\mathrm{M}+{ }^{56} \mathrm{Fe}^{3+}-2 \mathrm{H}^{+}\right]$. The intensity of the heavy iron isotopologue has been scaled by the natural abundance ratio of ${ }^{56} \mathrm{Fe} /{ }^{54} \mathrm{Fe}$ (divided by 15.7) so that the isotopologues overlap. 

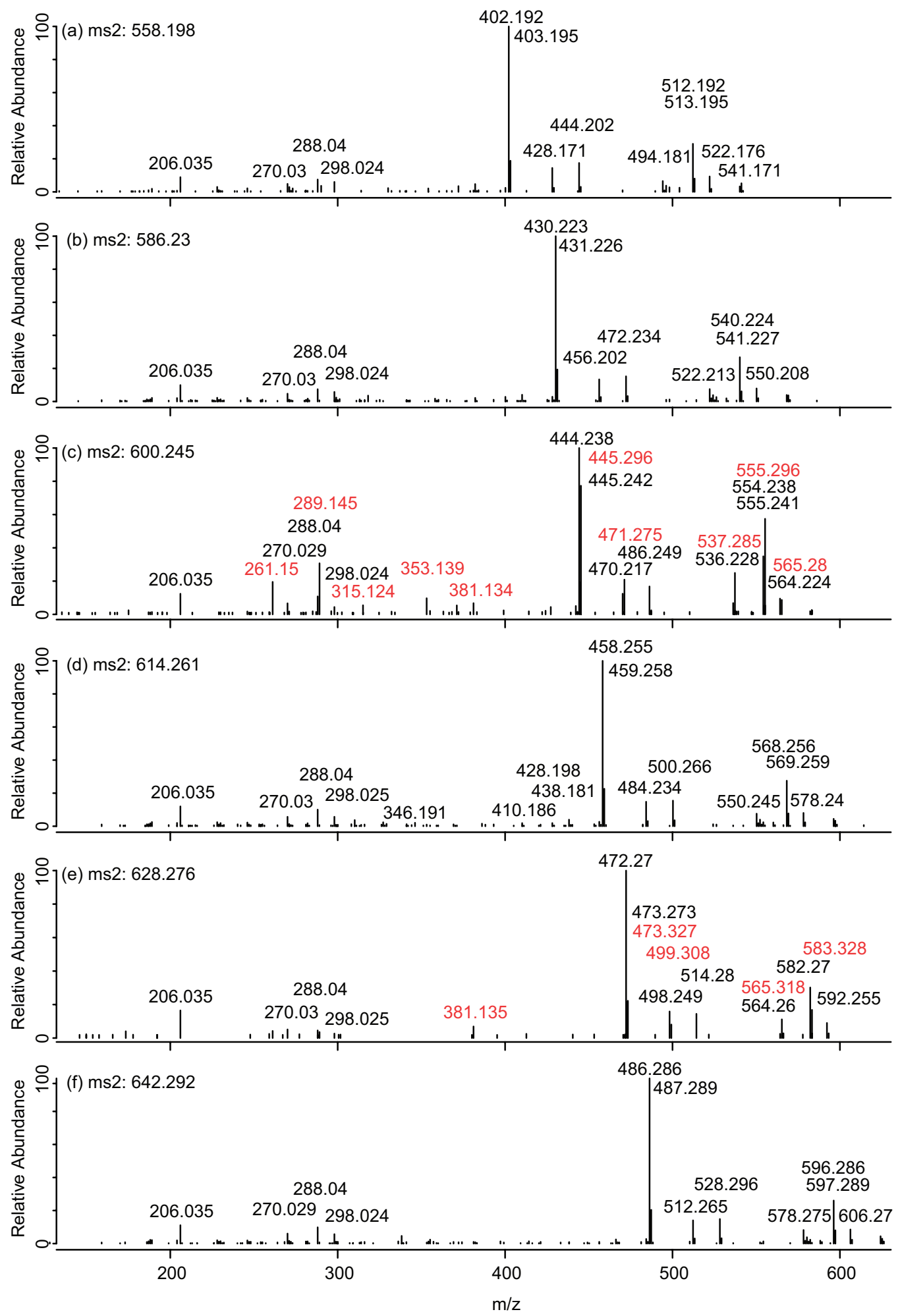

Figure 3.4: Positive mode MS2 spectra of the six identified Fe containing compounds. (a) $\mathrm{C}_{8}$ Synechobactin, (b) $\mathrm{C}_{10}$-synechobactin, (c) $\mathrm{C}_{11}$-synechobactin (d) $\mathrm{C}_{12}$-synechobactin (e) $\mathrm{C}_{13^{-}}$ synechobactin (f) $\mathrm{C}_{14}$-synechobactin. Red labels correspond to interferences from a coeluting ion (see supplementary information Figure 2). 
(a)

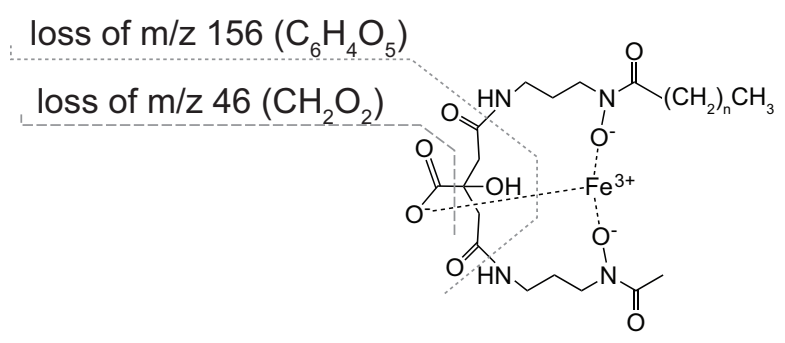

(b)

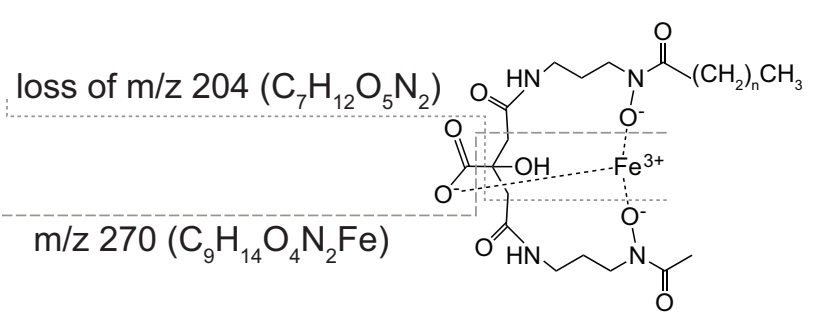

Figure 3.5: Characteristic fragmentation patterns of Fe-synechobactins. (a) Major fragmentations that result in the loss of the citrate head group. (b) Example of fragment pair that results in the symmetric loss of the short and long hydroxamate side chains. 
Table 3.1: Siderophores from Synechococcus sp. PCC 7002

\begin{tabular}{llll}
\hline $\begin{array}{l}\text { Monoisotopic } \\
\left({ }^{56} \mathrm{Fe} \text { form }\right)\end{array}$ & $\begin{array}{l}\text { Retention } \\
(\mathrm{min})\end{array}$ & $\begin{array}{l}\text { Time } \\
\text { farent } \\
\text { formula }\end{array}$ & \multicolumn{1}{c}{ ion } \\
Compound I.D.
\end{tabular}

*Previously described by Ito and Butler, 2005

$\dagger$ Putative ID based on detection of ${ }^{56} \mathrm{Fe}$ monoisotopic mass. ${ }^{54} \mathrm{Fe}$ isotopologue and MS2 peaks were below detection limit. 


\section{Chapter 3 Supplementary Information:}
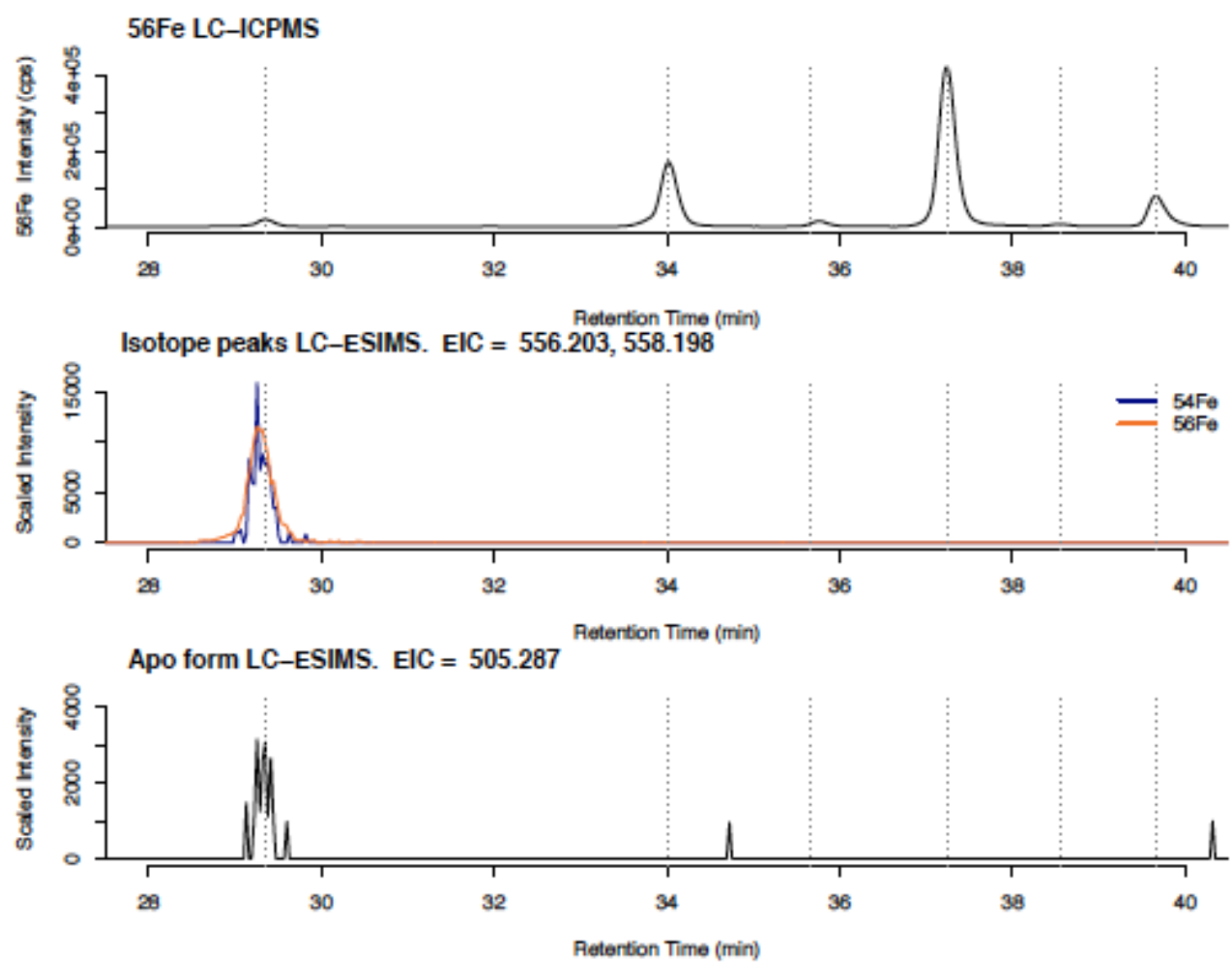

MS: $29.26 \mathrm{~min}$

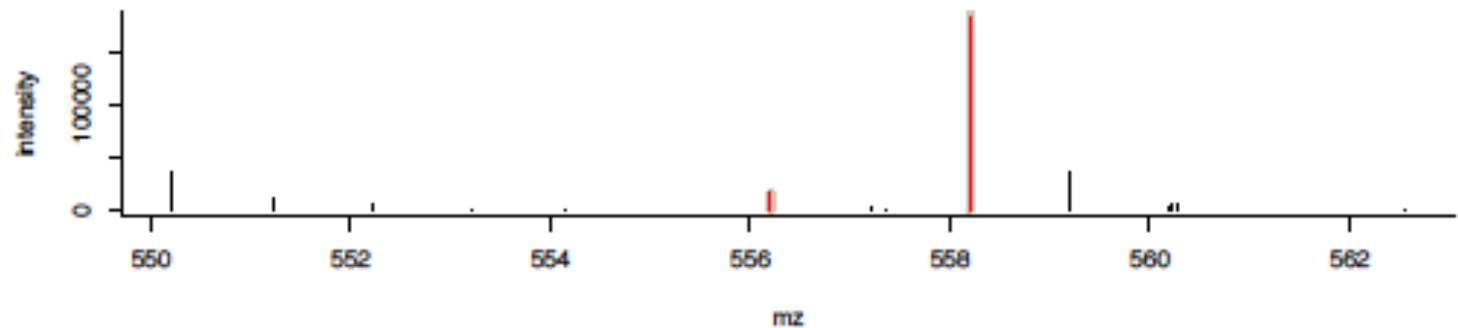

Figure SI-1a: Chromatograms and mass spectra from the major curated isotope pairs identified for each LC-ICPMS feature. (Top panel) ${ }^{56} \mathrm{Fe}$ LC-ICPMS chromatogram. Dotted lines indicate the retention times of the six synechobactins. (Second panel) extracted ion chromatograms from positive mode LC-ESIMS runs. Blue lines correspond to the light iron isotopologue $\left[\mathrm{M}^{54} \mathrm{Fe}^{3+}\right.$ $\left.2 \mathrm{H}^{+}\right]$that were identified by the isotope algorithm, and orange lines correspond to the heavy iron isotopologue $\left[\mathrm{M}+{ }^{56} \mathrm{Fe}^{3+}-2 \mathrm{H}^{+}\right]$. The intensity of the heavy iron isotopologue has been scaled by the natural abundance ratio of ${ }^{56} \mathrm{Fe} /{ }^{54} \mathrm{Fe}$ (divided by 15.7) so that the isotopologues overlap. (Third panel) extracted ion chromatograms of the Apo (iron free) monoisotopic $\mathrm{m} / \mathrm{z}\left[\mathrm{M}+\mathrm{H}^{+}\right]$. (Bottom panel) MS1 spectra of the Fe-complex. The red lines highlight the peaks corresponding to the ${ }^{54} \mathrm{Fe}$ and ${ }^{56} \mathrm{Fe}$ isotopologues that are plotted in the second panel. The theoretical Fe isotope pattern (Mass $\mathrm{M}_{1}-\mathrm{M}_{2}=1.995 \mathrm{~m} / \mathrm{z}$, Intensity $\mathrm{M}_{1} / \mathrm{M}_{2}=15.7$ ) for the ion pair is displayed as gray bars. 


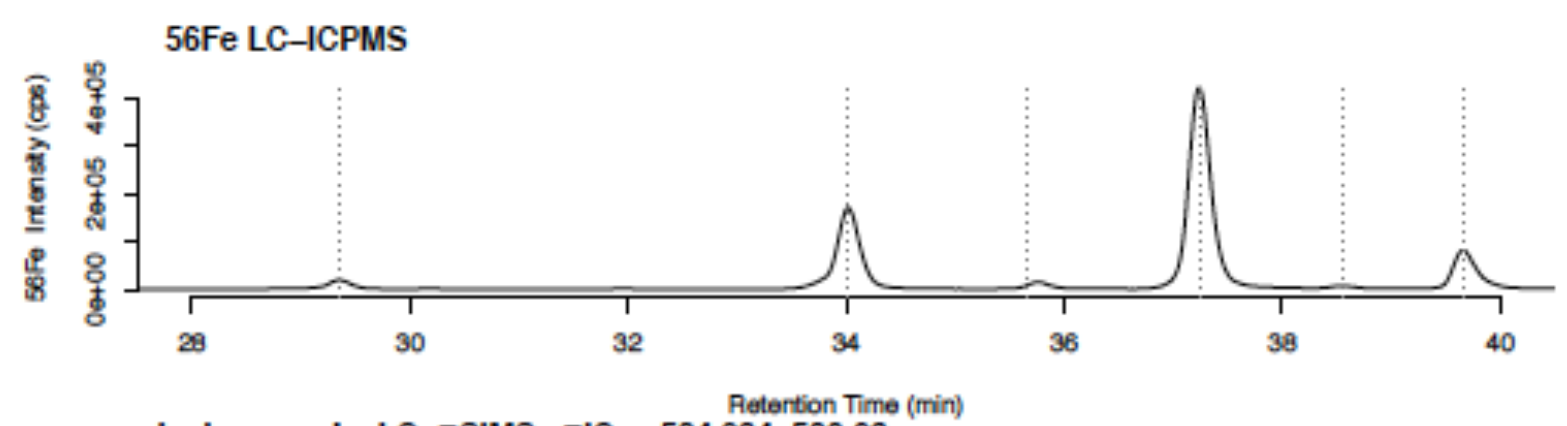

Isotope peaks LC-ESIMS. EIC $=584.234,586.23$

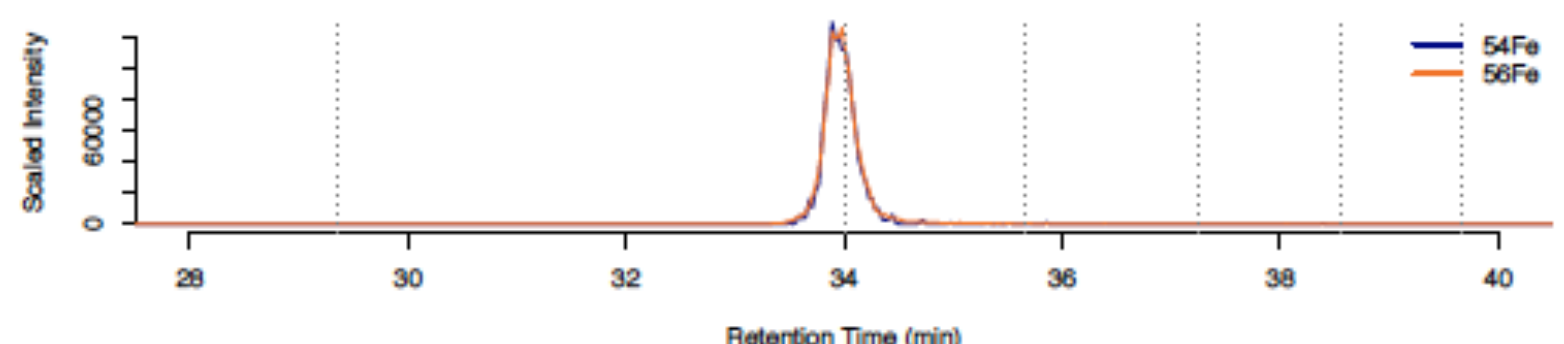

Apo form LC-ESIMS. EIC $=533.318$

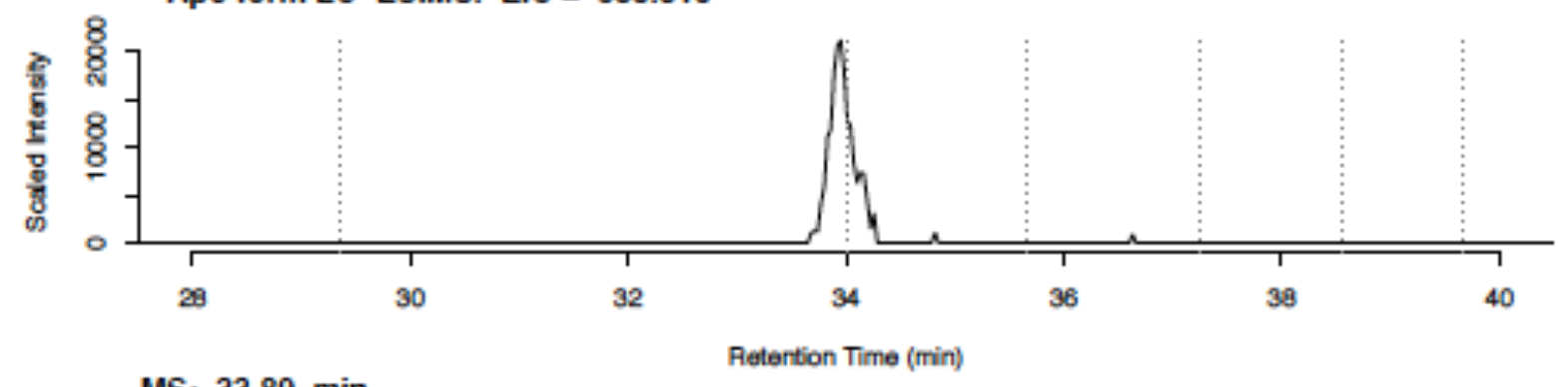

MS: $33.89 \mathrm{~min}$

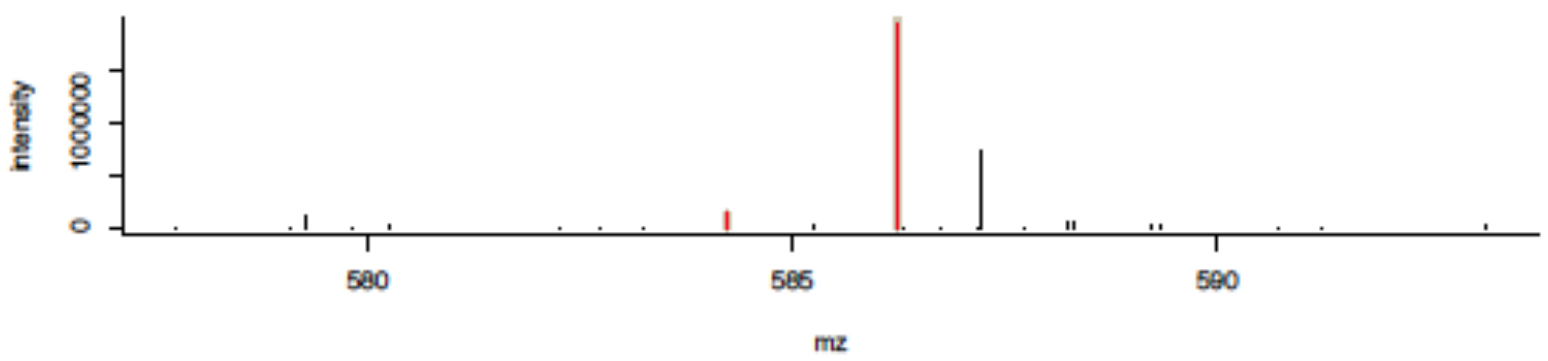

Figure SI-1b: Chromatograms and mass spectra from the major curated isotope pairs identified for each LC-ICPMS feature. Panels are the same are described in the legend for figure SI-1a. 

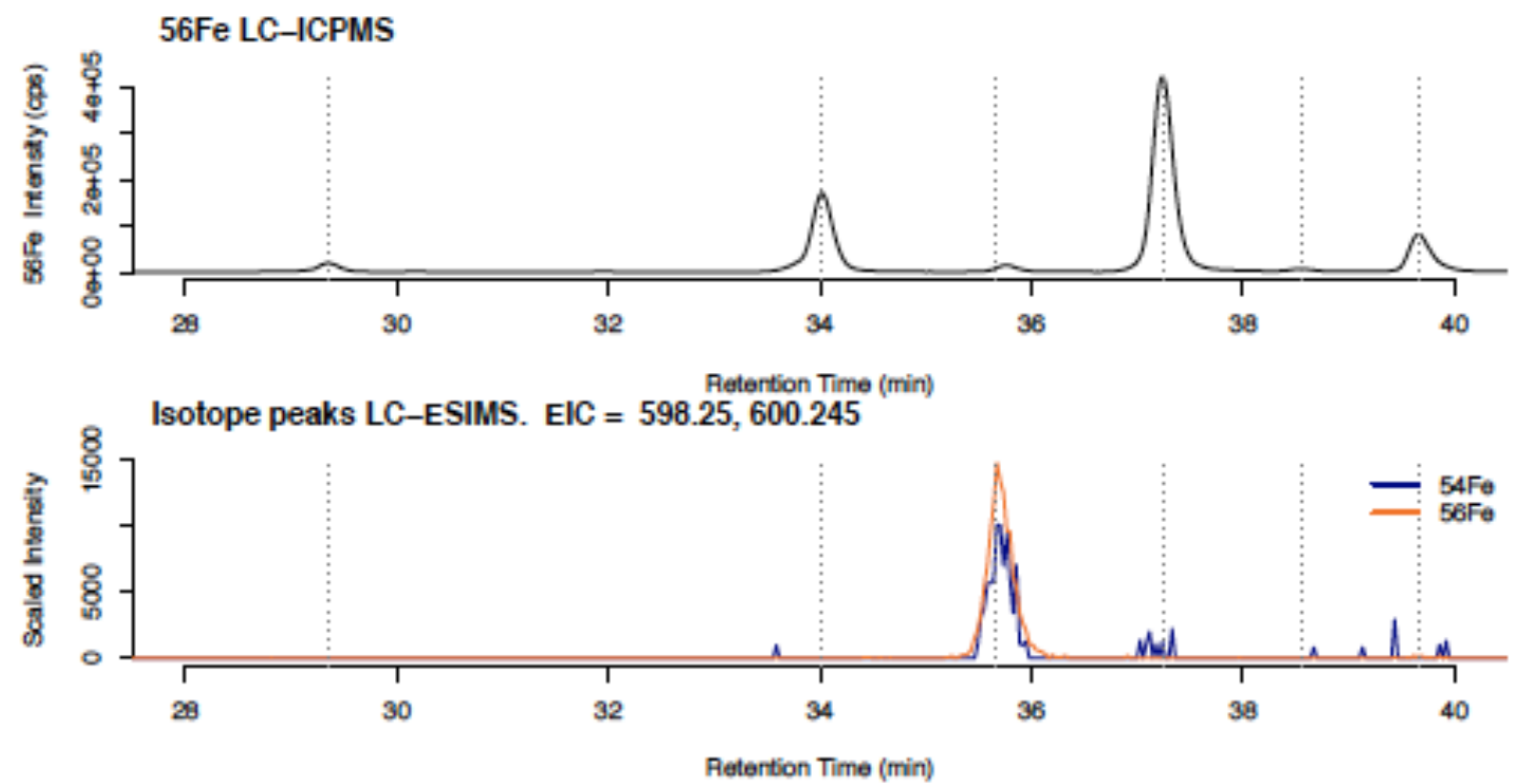

Apo form LC-ESIMS. EIC $=547.334$

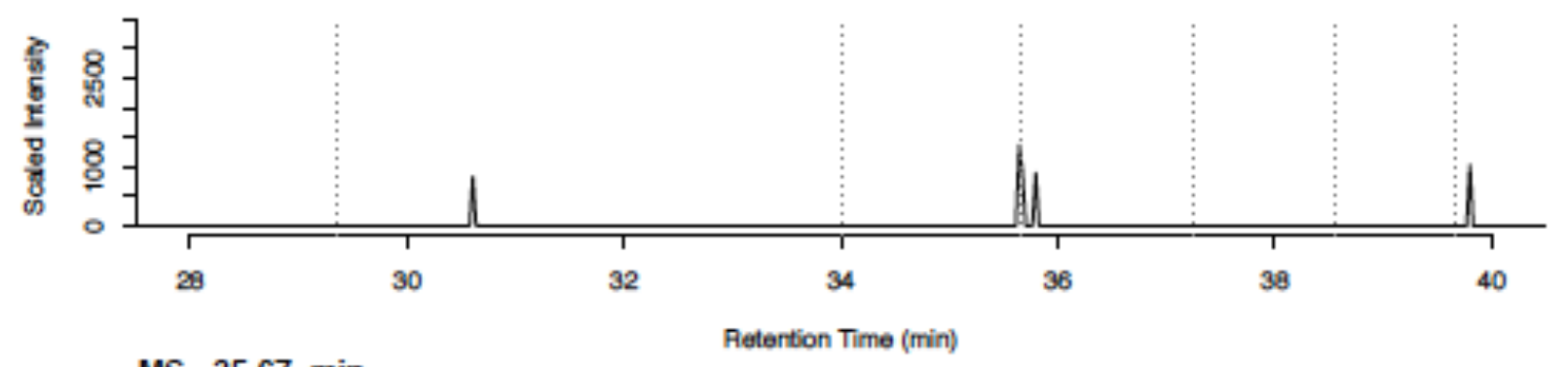

MS: $35.67 \mathrm{~min}$

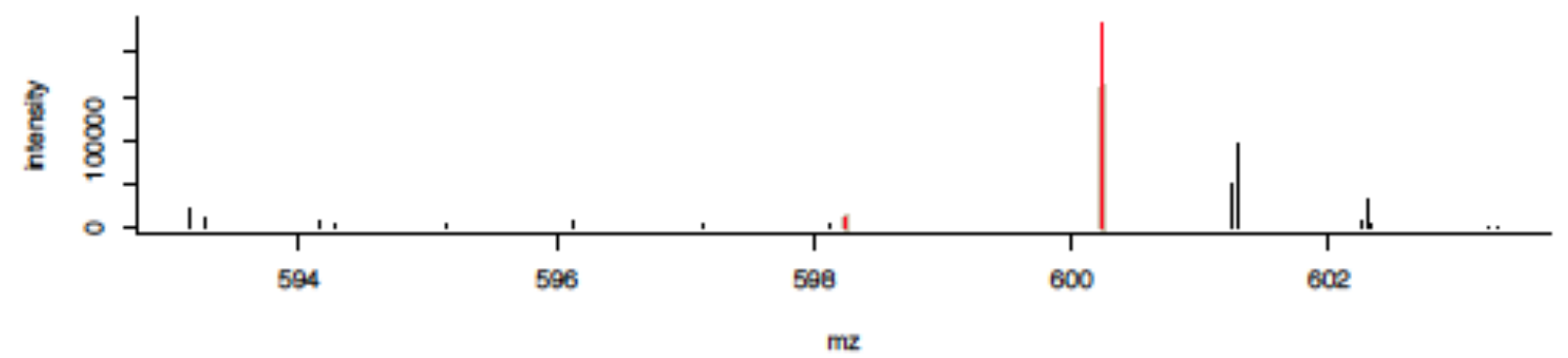

Figure SI-1c: Chromatograms and mass spectra from the major curated isotope pairs identified for each LC-ICPMS feature. Panels are the same are described in the legend for figure SI-1a. 


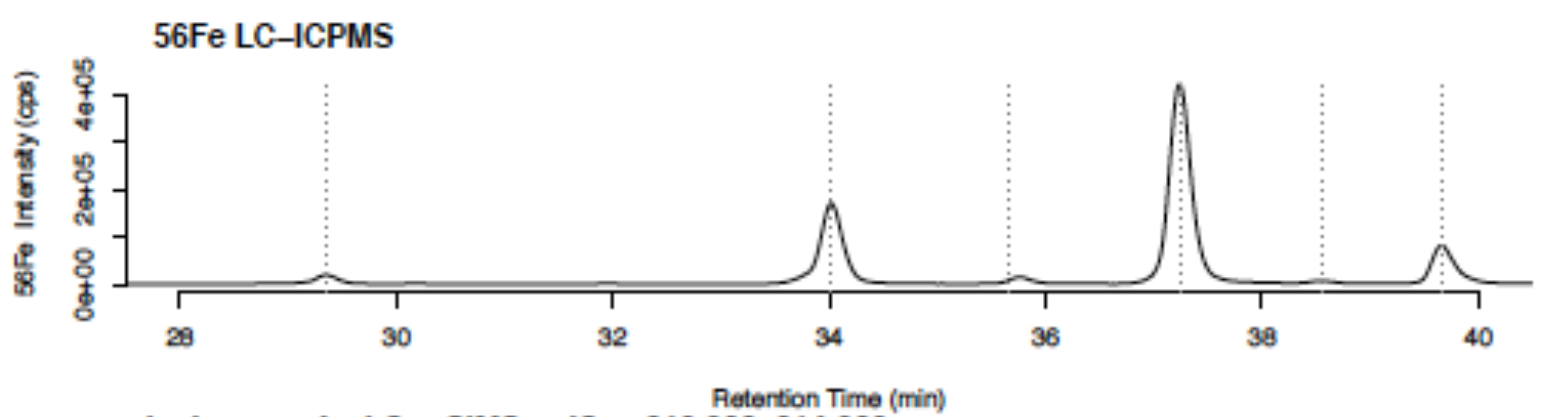

Isotope peaks LC-ESIMS. EIC $=612.266,614.262$
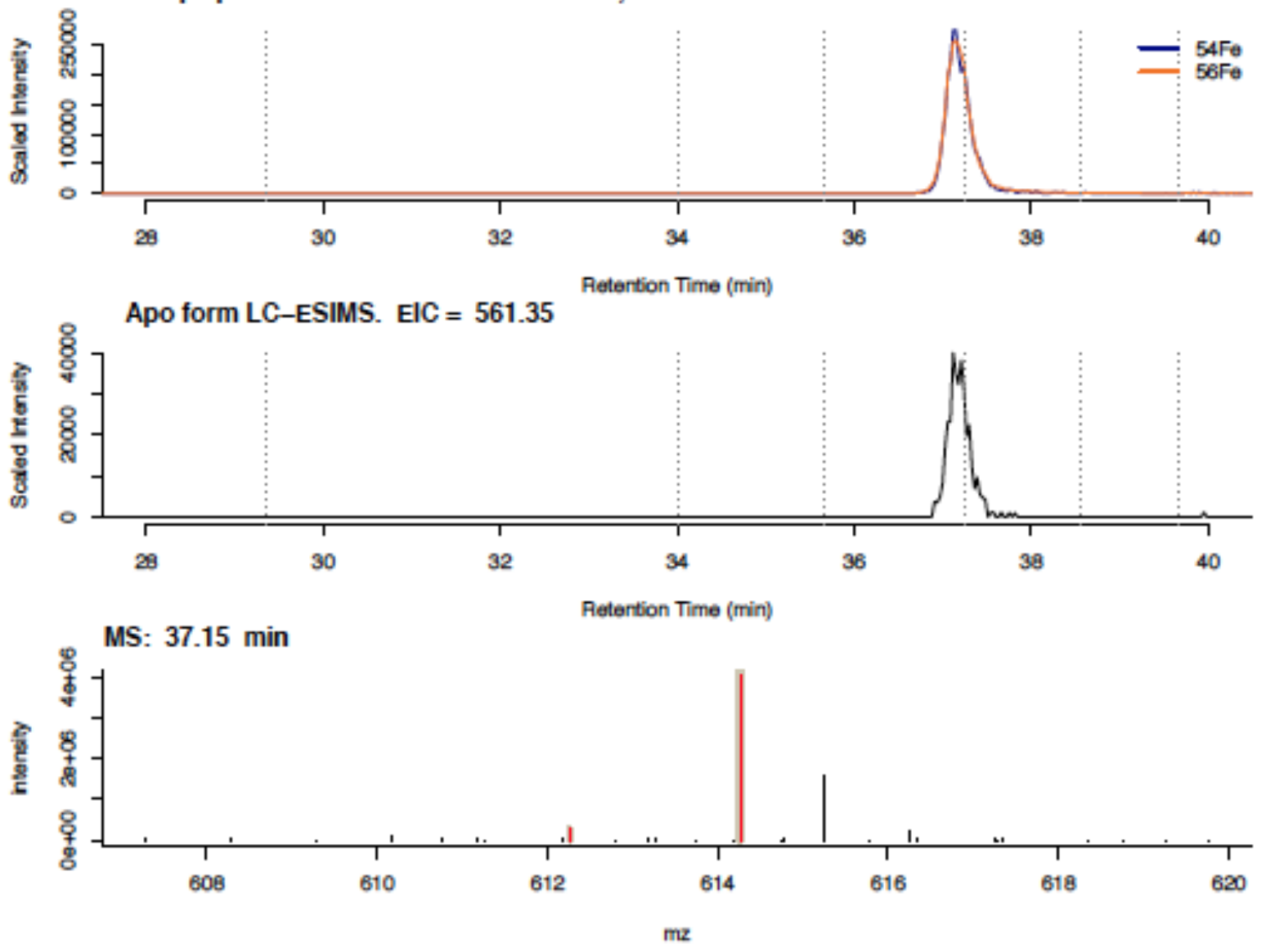

Figure SI-1d: Chromatograms and mass spectra from the major curated isotope pairs identified for each LC-ICPMS feature. Panels are the same are described in the legend for figure SI-1a. 


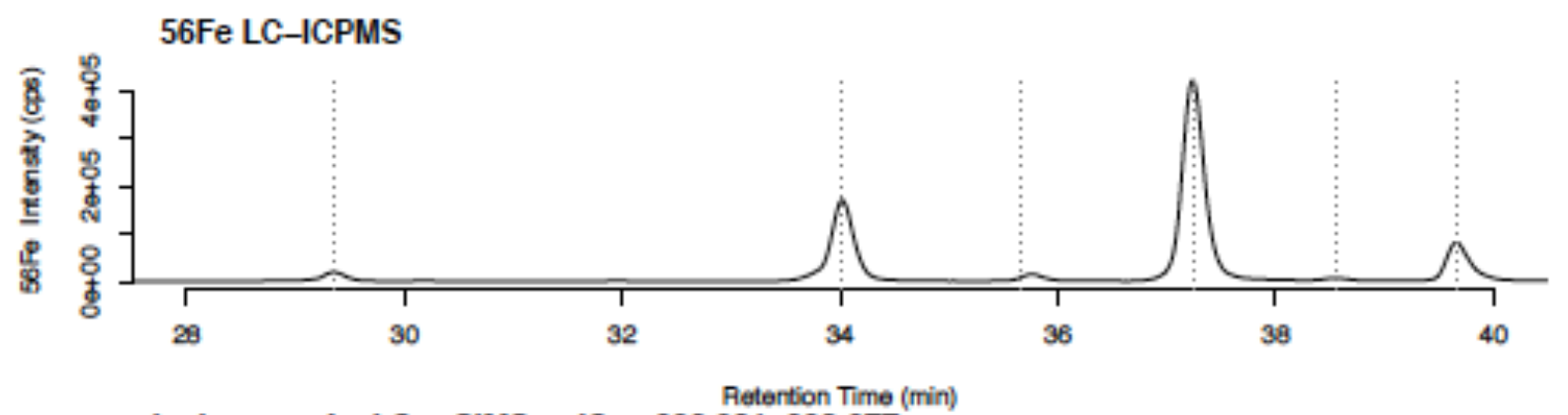

Isotope peaks LC-ESIMS. EIC $=626.281,628.277$

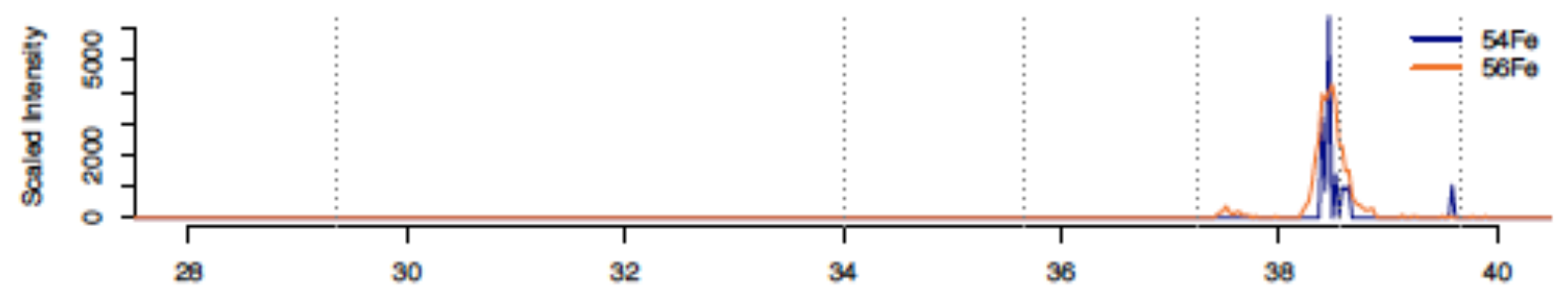

Apo form LC-ESIMS. EIC $=575.365$

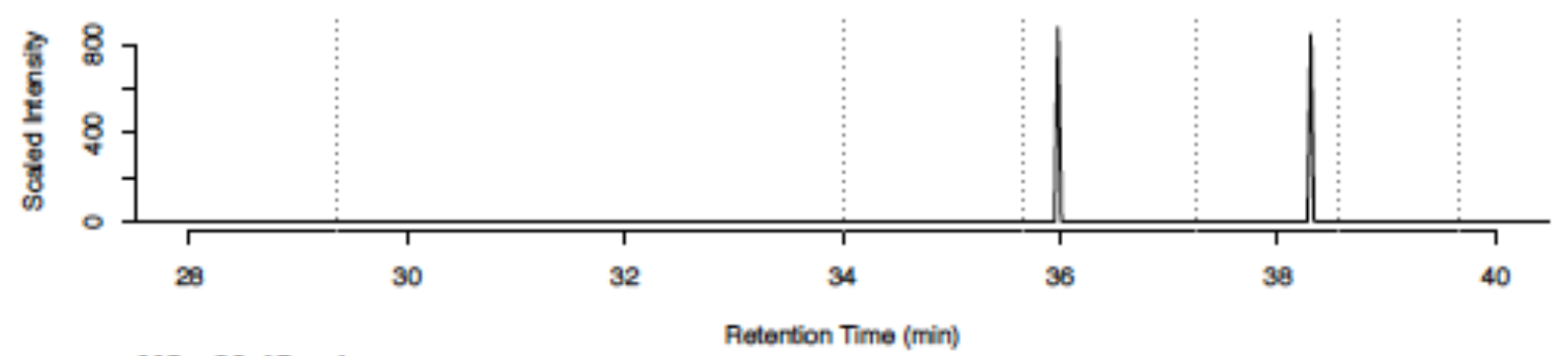

MS: $38.45 \min$

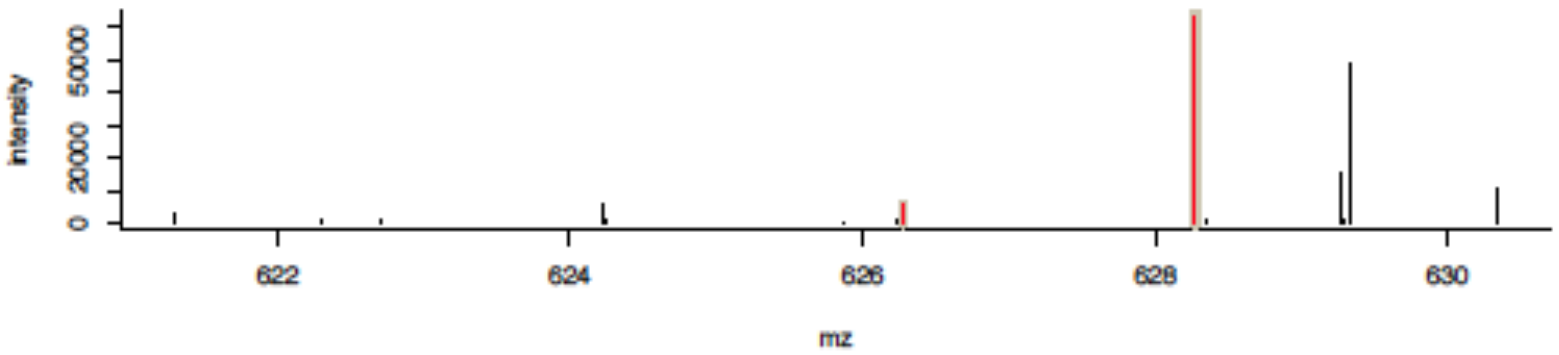

Figure SI-1e: Chromatograms and mass spectra from the major curated isotope pairs identified for each LC-ICPMS feature. Panels are the same are described in the legend for figure SI-1a. 


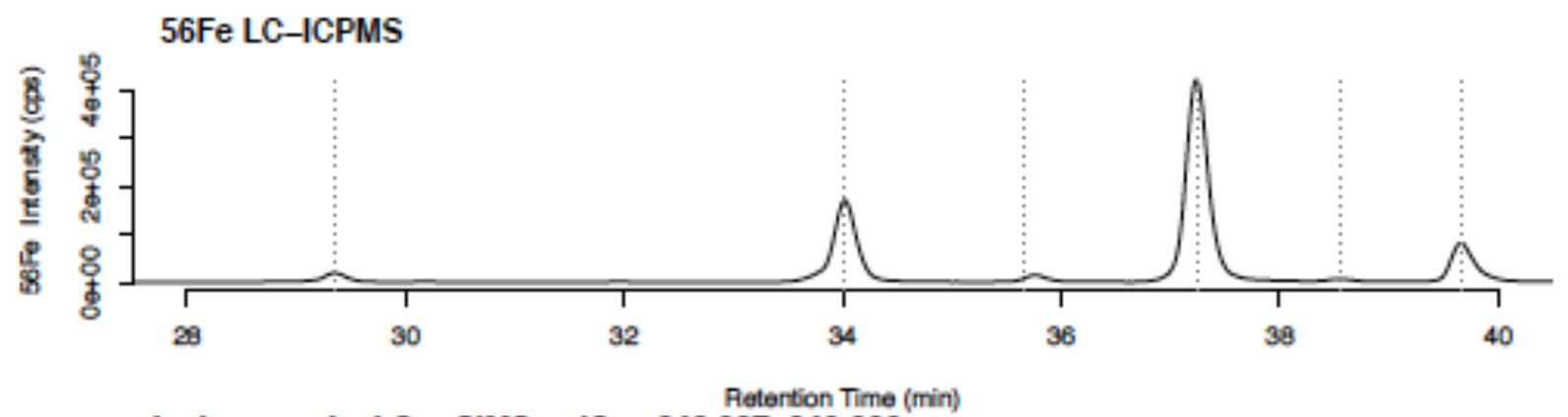

Isotope peaks LC-ESIMS. EIC $=640.297,642.292$

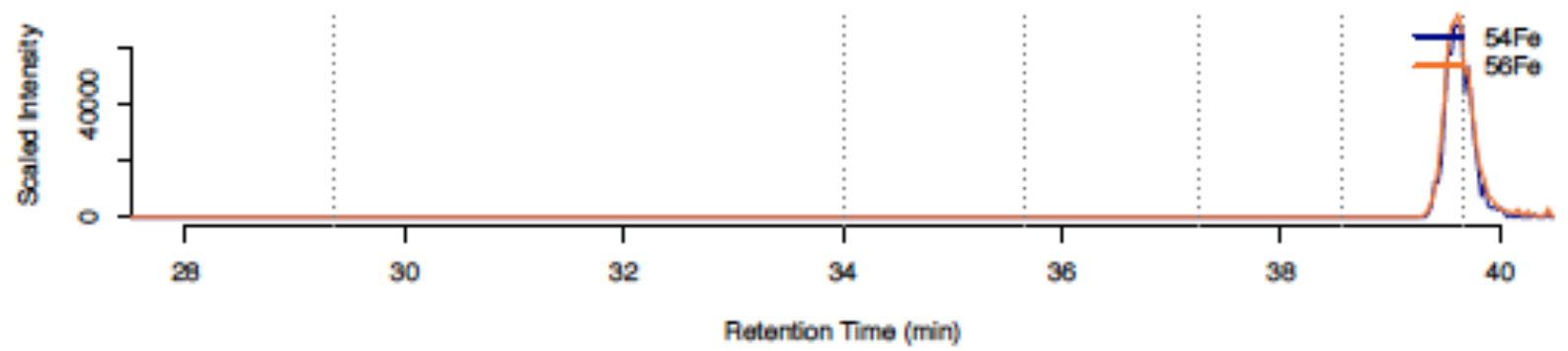

Apo form LC-ESIMS. EIC $=589.381$
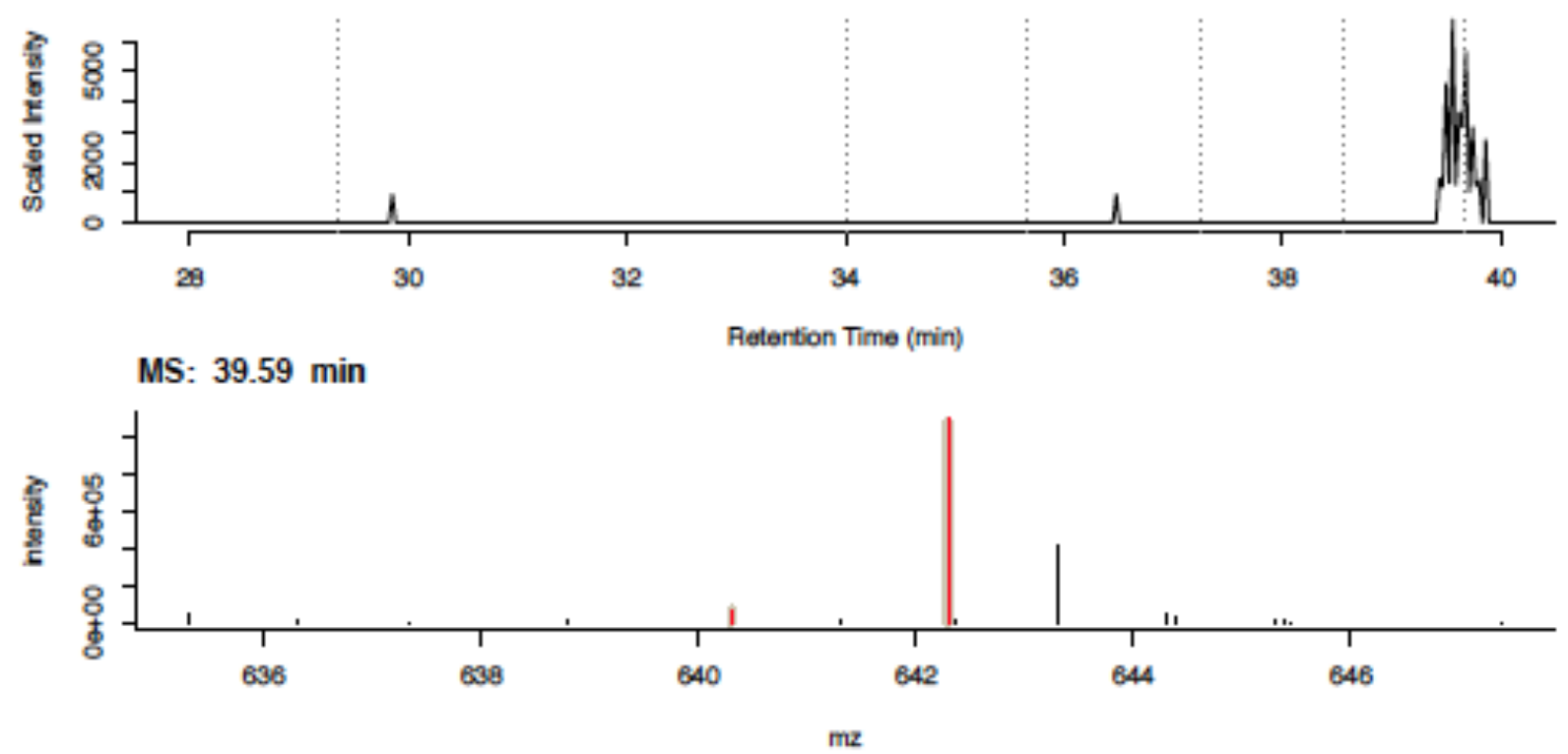

Figure SI-1f: Chromatograms and mass spectra from the major curated isotope pairs identified for each LC-ICPMS feature. Panels are the same are described in the legend for figure SI-1a. 
(a) ms2: 533.318 (Apo form, synechobactin $B\left(C_{10}\right)$, positive mode)

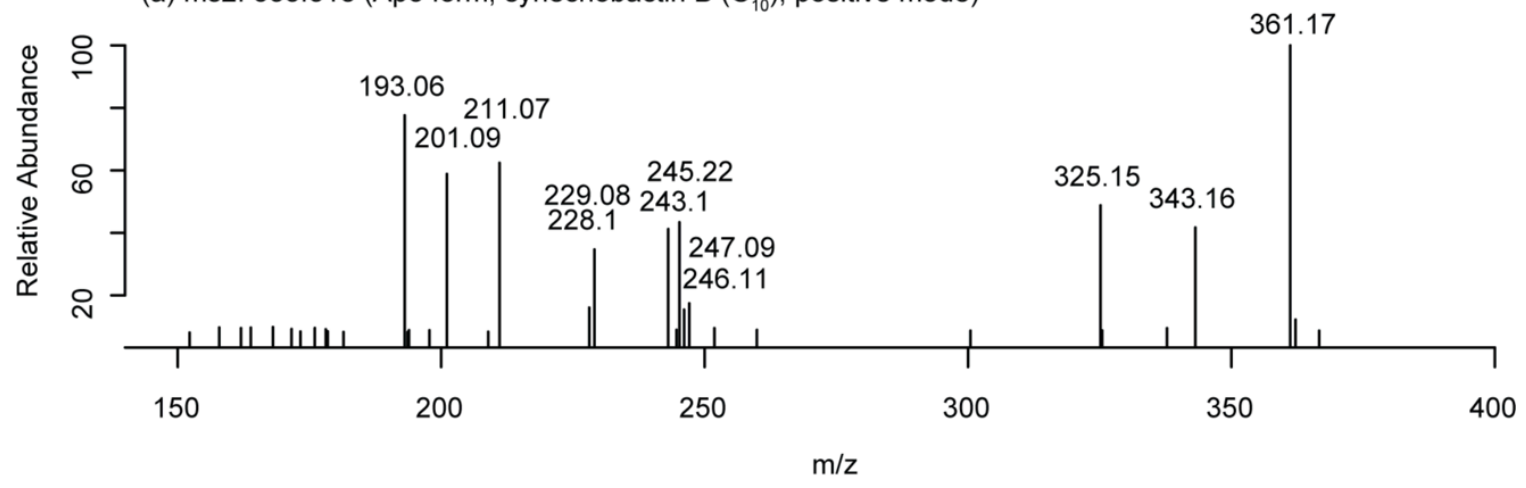

(b) ms2: 561.35 (Apo form, synechobactin $A\left(C_{12}\right)$, positive mode)

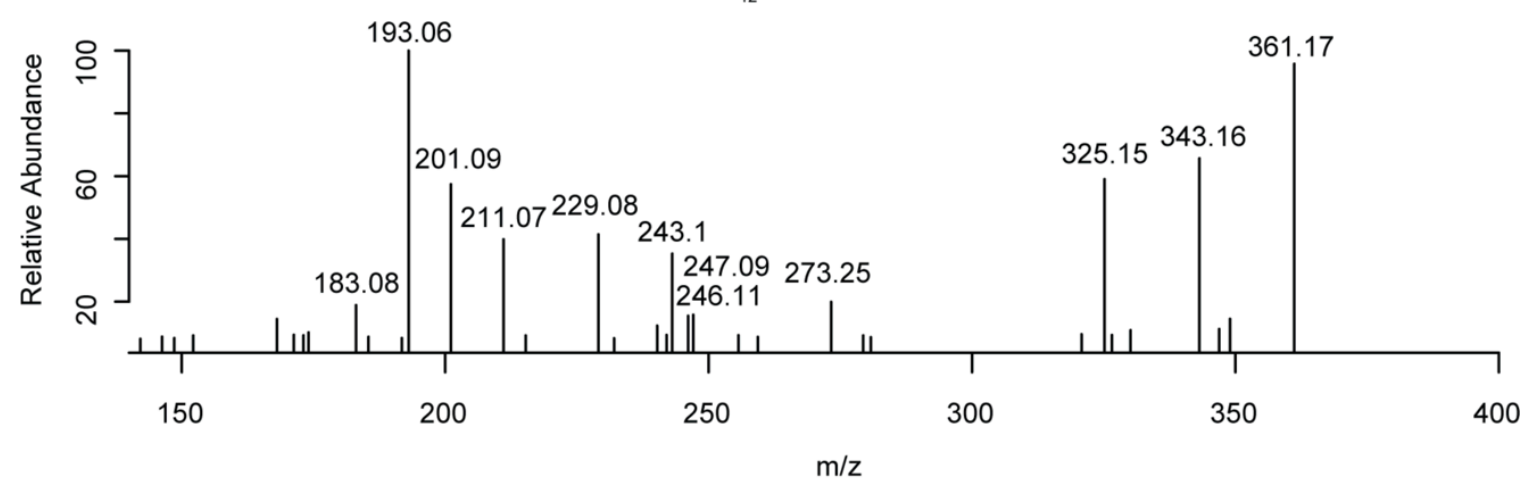

(c) ms2: $612.24\left({ }^{56} \mathrm{Fe}\right.$ synechobactin $\mathrm{A}\left(\mathrm{C}_{12}\right)$, negative mode)

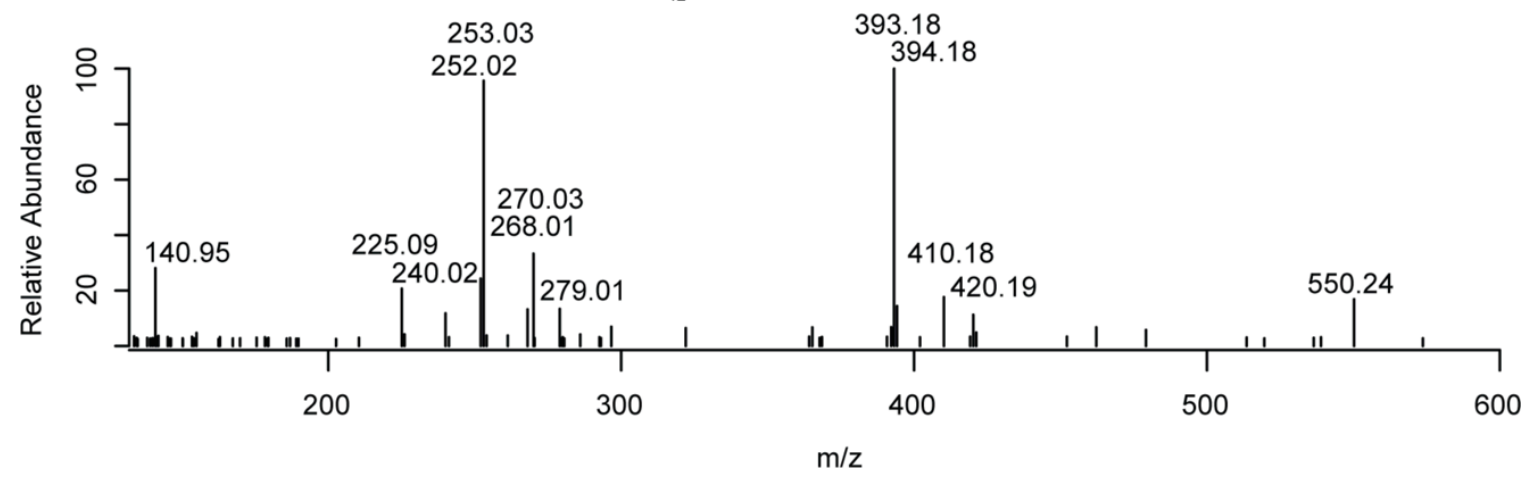

Figure SI-2: (a-b) Top and middle panels show the positive mode MS2 spectra of apo (iron-free) form $\left[\mathrm{M}+\mathrm{H}^{+}\right]$of synechobactin $\mathrm{B}$ and synechobactin A. (c) Bottom panel shows the negative mode MS2 spectra of the iron bound form of synechobactin A $\left[\mathrm{M}+{ }^{56} \mathrm{Fe}-4 \mathrm{H}^{+}\right]$. Data was collected on the low mass resolution ion trap detector using an isolation window of $\pm 2 \mathrm{~m} / \mathrm{z}$ 
Sources of interfering ions in synechobactin MS2 spectra:
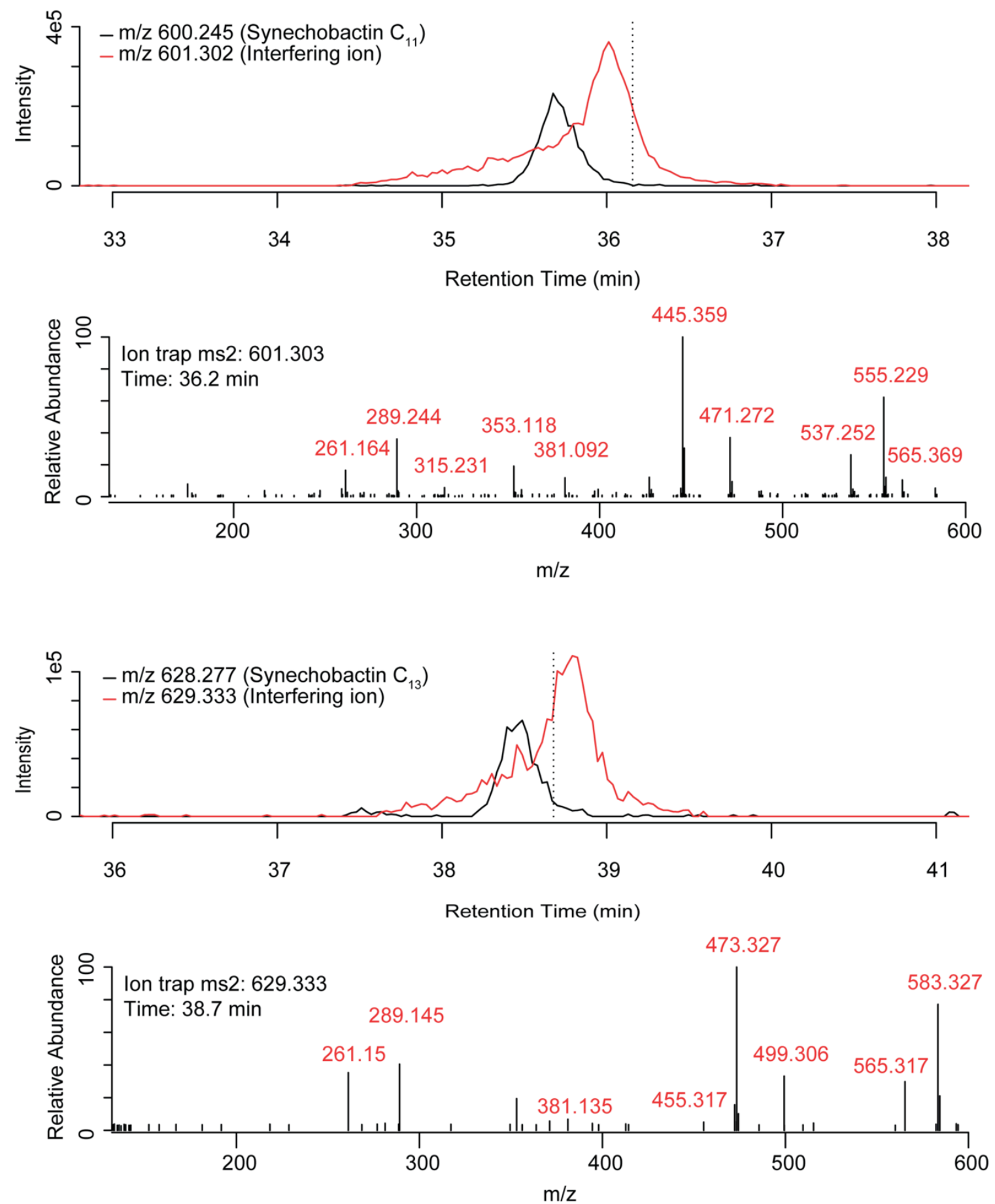

Figure SI-3: EIC and MS2 spectra of interfering ions. The upper panel shows the extracted ion chromatogram (EIC) of synechobactins (a) $\mathrm{C}_{11}$ and (b) $\mathrm{C}_{13}$ in black and the EIC of the coeluting ion that interferes with the MS2 spectra in red. The vertical dashed lines indicate the retention time at which MS2 spectra of the interfering ion (shown in the lower panel) was collected on the low mass resolution ion trap detector using an isolation window of $\pm 2 \mathrm{~m} / \mathrm{z}$. The red labels in the lower panels correspond to the interfering MS2 ions that appear in the spectra in Figure 4 of the text. 


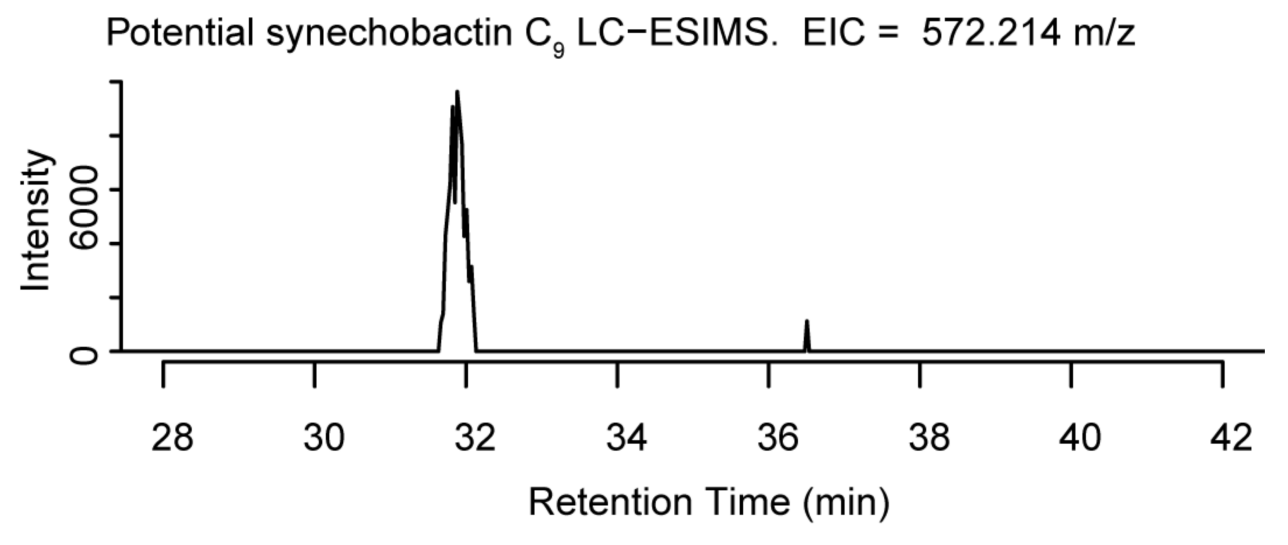

Potential synechobactin $C_{16}$ LC-ESIMS. EIC $=670.324 \mathrm{~m} / \mathrm{z}$

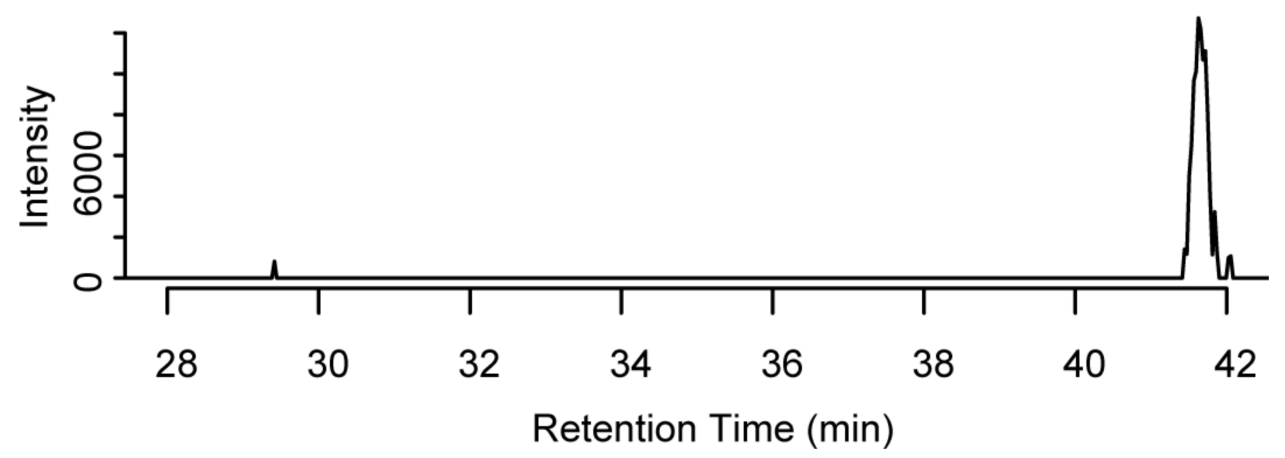

Figure SI-4: Extracted ion chromatograms (EIC) of m/z 670.323 and 572.214, which correspond to the ${ }^{56} \mathrm{Fe}$ monoisotopic mass $\left[\mathrm{M}+{ }^{56} \mathrm{Fe}^{3+}-2 \mathrm{H}^{+}\right]$of synechobactin $\mathrm{C}_{9}$ and synechobactin $\mathrm{C}_{16}$. 
(a) ${ }^{56} \mathrm{Fe}$ abundance: LC-ICPMS

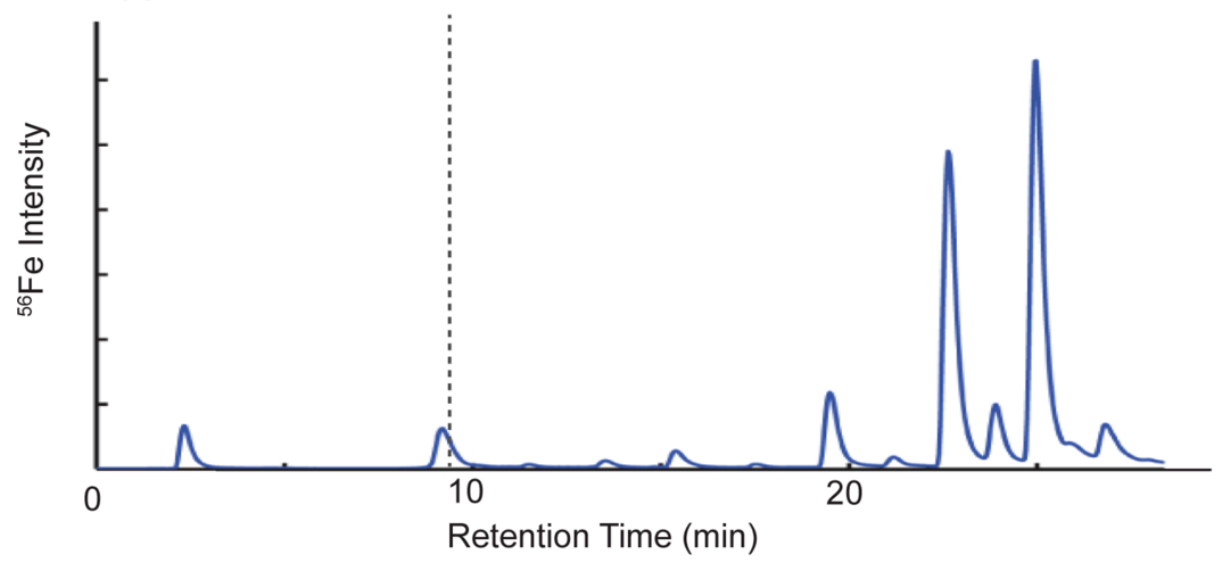

(b) Fe Isotopologues LC-ESIMS (schizokinen)

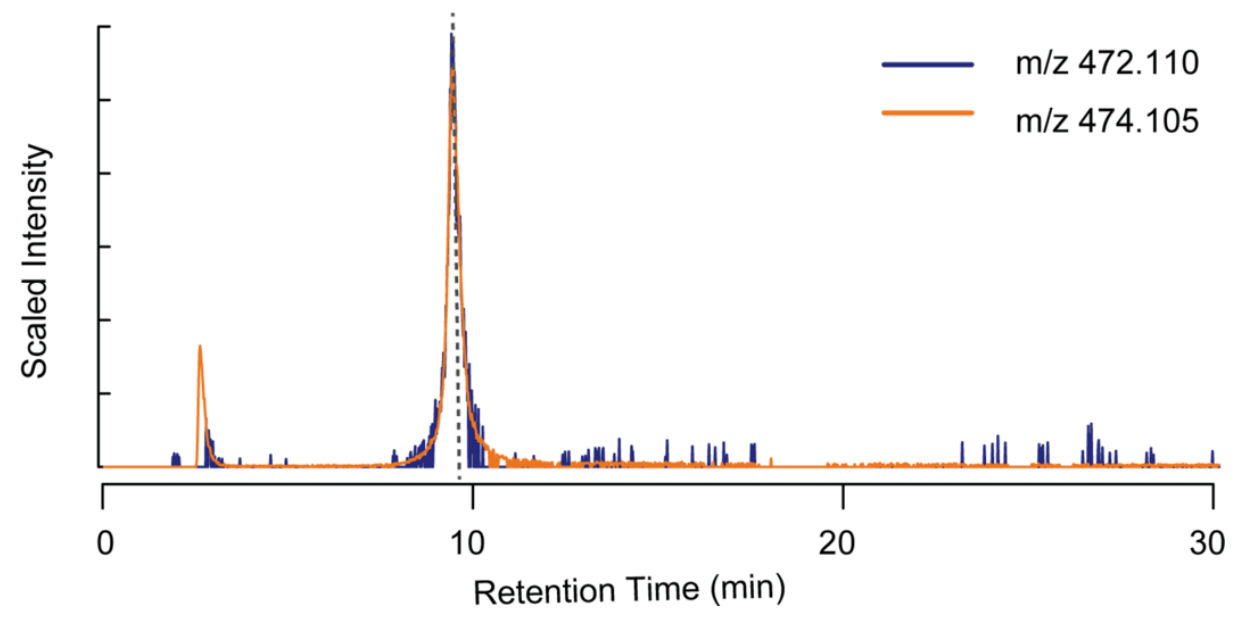

Figure SI-5: Schizokinen production by Synechococcus sp. PCC 7002. LC-MS chromatograms of Synechoccoccus sp. PCC 7002 media extract collected during a previous study (Boiteau et al., 2013). (a) ${ }^{56} \mathrm{Fe}$ LC-ICPMS chromatogram. The peak at 10 minutes corresponds to schizokinen produced by Synechococcus sp PCC 7002 in this experiment (b) extracted ion chromatograms from positive mode LC-ESIMS run. The blue line correspond to the light iron isotopologue of schizokinen $\left[\mathrm{M}+{ }^{54} \mathrm{Fe}^{3+}-2 \mathrm{H}^{+}\right]$that were identified by the isotope algorithm, and the orange line corresponds to the heavy iron isotopologue $\left[\mathrm{M}+{ }^{56} \mathrm{Fe}^{3+}-2 \mathrm{H}^{+}\right]$. The intensity of the heavy iron isotopologue has been scaled by the natural abundance ratio of ${ }^{56} \mathrm{Fe} /{ }^{54} \mathrm{Fe}$ (divided by 15.7 ) so that the isotopologues overlap. Compounds were separated with a 20 minute chromatographic gradient from $\mathrm{H}_{2} \mathrm{O}$ to $\mathrm{MeOH}$ using $5 \mathrm{mM}$ ammonium formate as a buffer and a $3 \mu \mathrm{m}$ particle size $2.1 \times 150 \mathrm{~mm} \mathrm{C18}$ column (Hamilton). 
Table SI-1: Complete list of curated isotope pairs identified by algorithm

\begin{tabular}{|c|c|c|c|c|c|}
\hline $\begin{array}{l}\text { Retention } \\
\text { Time (min) }\end{array}$ & $\begin{array}{l}{ }^{54} \mathrm{FeL} \\
{\left[\mathrm{M}+{ }^{54} \mathrm{Fe}^{3}\right.} \\
\left.{ }^{+}-2 \mathbf{H}^{+}\right] \\
\mathrm{m} / \mathbf{z}\end{array}$ & $\begin{array}{l}\text { Max }{ }^{54} \mathrm{FeL} \\
\text { intensity } \\
\text { (cps) }\end{array}$ & $\begin{array}{l}{ }^{56} \mathrm{FeL} \\
{\left[\mathrm{M}+{ }^{56} \mathrm{Fe}^{3}\right.} \\
\left.{ }^{+}-2 \mathrm{H}^{+}\right] \\
\mathrm{m} / \mathrm{z}\end{array}$ & $\begin{array}{l}\text { Max }{ }^{56} \mathrm{FeL} \\
\text { intensity } \\
\text { (cps) }\end{array}$ & $\begin{array}{l}\text { Detected with } \\
\text { narrow } \\
\text { intensity ratio } \\
\text { tolerance } \\
( \pm 25 \%) ?\end{array}$ \\
\hline 29.3 & 556.203 & 15865 & 558.198 & 181282 & $\mathrm{Y}$ \\
\hline 29.3 & 557.206 & 2958 & 559.202 & 39739 & $\mathrm{Y}$ \\
\hline 33.9 & 584.235 & 128678 & 586.230 & 1922865 & $\mathrm{Y}$ \\
\hline 33.6 & 656.292 & 3707 & 658.287 & 66731 & $\mathrm{Y}$ \\
\hline 33.9 & 585.238 & 22767 & 587.234 & 560587 & $\mathrm{Y}$ \\
\hline 33.9 & 583.242 & 1075 & 585.238 & 26840 & \\
\hline 33.9 & 606.217 & 1255 & 608.211 & 25138 & \\
\hline 33.9 & 645.287 & 2925 & 647.282 & 31892 & \\
\hline 34.0 & 1140.502 & 1348 & 1142.499 & 20312 & $\mathrm{Y}$ \\
\hline 35.7 & 598.250 & 10117 & 600.245 & 232330 & $\mathrm{Y}$ \\
\hline 35.6 & 599.253 & 3057 & 601.249 & 31863 & $\mathrm{Y}$ \\
\hline 35.8 & 628.261 & 1122 & 630.256 & 22456 & \\
\hline 37.1 & 612.266 & 274030 & 614.261 & 4023540 & $\mathrm{Y}$ \\
\hline 37.1 & 613.270 & 66696 & 615.263 & 1588548 & $\mathrm{Y}$ \\
\hline 37.2 & 673.318 & 5209 & 675.314 & 84421 & \\
\hline 37.2 & 1213.593 & 7249 & 1215.588 & 107493 & $\mathrm{Y}$ \\
\hline 37.1 & 928.528 & 2713 & 930.523 & 35734 & $\mathrm{Y}$ \\
\hline 37.1 & 1196.565 & 10849 & 1198.561 & 179926 & $\mathrm{Y}$ \\
\hline 37.1 & 1197.570 & 6392 & 1199.564 & 103510 & $\mathrm{Y}$ \\
\hline 37.2 & 629.291 & 3316 & 631.287 & 51143 & $\mathrm{Y}$ \\
\hline 37.1 & 1214.596 & 4136 & 1216.592 & 66421 & \\
\hline 37.1 & 1225.521 & 5997 & 1227.515 & 76948 & $\mathrm{Y}$ \\
\hline 37.2 & 611.277 & 4396 & 613.270 & 52233 & \\
\hline 37.1 & 674.326 & 2953 & 676.321 & 43493 & \\
\hline 37.1 & 1243.603 & 1442 & 1245.599 & 14531 & \\
\hline 38.5 & 626.282 & 6402 & 628.276 & 62850 & \\
\hline 39.6 & 640.297 & 68580 & 642.292 & 1091913 & $\mathrm{Y}$ \\
\hline 39.5 & 701.350 & 1098 & 703.345 & 14075 & \\
\hline 39.6 & 641.301 & 20860 & 643.296 & 425004 & \\
\hline
\end{tabular}




\section{Appendix 3 Marinobactins from Costa Rica Upwelling Dome heterotrophs}

The LC-ICPMS-ESIMS based siderophore screening method described in this chapter opens new opportunities for high throughput identification of siderophores produced by marine microbes in culture. Such analyses will expand our understanding of the taxonomic groups that are capable of producing specific siderophores and expand our libraries of known siderophores. This work is necessary to connect the masses and fragmentation spectra of siderophores identified in the environment to specific compounds, biosynthetic genes, and eventually producers and consumers.

To this end, we investigated siderophores produced by copiotrophic marine bacterial isolates from the iron-deficient Costa Rica Upwelling Dome. Currently, heterotrophic siderophores in seawater are typically identified by using the colorometric CAS assay to screen fractions of organic carbon that have been separated by HPLC, which requires nanomole quantities of unbound ligand [Schwyn and Neilands, 1987; Neilands and Nakamura, 1991]. The LC-ICPMSESIMS pipeline requires smaller sample sizes and shorter screening times with a lower detection limit than CAS based methods for characterizing marine Fe ligands.

The Webb laboratory at the University of Southern California has created a library of marine heterotrophs from the Costa Rica Upwelling Dome cruise in 2005. This site is characterized by seasonal upwelling of nutrient rich waters that results in enhanced primary productivity. While most upwelling regimes are dominated by fast-growing eukaryotes such as diatoms, the Costa Rica Upwelling Dome (CRUD) is characterized by high concentrations of the cyanobacteria Synechococcus, and thus provides a unique environment to study metal-organism interactions [Saito et al., 2005]. The isolated heterotroph strains have been sequenced and screened for siderophore production using the CAS assay, the Arnow test, and the Czaky assay. These colorimetric tests indicate the presence or absence of general iron ligands, catecholate, and hydroxamate siderophores respectively [Neilands and Nakamura, 1991]. The importance of siderophores in the Costa Rican Dome ecosystem is highlighted by the prevalence of siderophore producers: of 867 different heterotroph strains isolated from this environment, 340 were capable of making siderophores [Krey, 2008]. 
Of the 13 heterotrophic strains were analyzed, two strains (Alteromonas 2E5 and Pseudoalteromonas 2E11) were found to produce suites of marinobactins, amphiphilic hydroxamate siderophores originally identified in Marinobacter species strains DS40M6 and DS40M8 [Martinez et al., 2000] (Fig. A3.1). In addition to the four previously characterized marinobactins, we found two novel compounds that were putatively identified as unsaturated forms of marinobactin A. It is notable that Alteromonas 2E5 and Pseudoalteromonas 2E11 were collected from the same water mass in samples taken $2 \mathrm{~m}$ apart. These findings expand the known producers of marinobactins and suggest that positive selection pressure for iron acquisition in the marine environment drives the horizontal gene transfer of marinobactin biosynthetic pathways. Future work will determine the sequence similarity between marinobactin biosynthetic gene clusters in Alteromonas 2E5 and Pseudoalteromonas 2E11, and will look for nearby elements involved in genetic transposition or integration.

\section{Methods:}

Heterotrophic strains were grown in artificial seawater media. To make the media, $25.95 \mathrm{~g} \mathrm{NaCl}$, $3 \mathrm{~g} \mathrm{MgSO}_{4} * 7 \mathrm{H}_{2} \mathrm{O}, 2 \mathrm{~g} \mathrm{MgCl}_{2} * 6 \mathrm{H}_{2} \mathrm{O}, 0.12 \mathrm{~g} \mathrm{CaCl}_{2} * 2 \mathrm{H}_{2} \mathrm{O}$, and $10 \mathrm{~mL}$ of $1 \mathrm{M} \mathrm{NH}_{4} \mathrm{Cl}$ were added to $400 \mathrm{~mL} \mathrm{H}_{2} \mathrm{O}$. In a separate beaker, $0.8 \mathrm{~g} \mathrm{~K}_{2} \mathrm{HPO}_{4}$ and $0.2 \mathrm{~g} \mathrm{H}_{2} \mathrm{PO}_{4}$ were added to $400 \mathrm{~mL} \mathrm{H}_{2} \mathrm{O}$. The $\mathrm{pH}$ of both solutions was adjusted to $\mathrm{pH} 8$ and the solutions were autoclaved. Once cooled, the solutions were combined. Sterile $0.5 \%$ glycerol was added as a carbon source, along with $800 \mu \mathrm{L}$ of $\mathrm{Na}_{2} \mathrm{MO}_{4} * 2 \mathrm{H}_{2} \mathrm{O}(0.03 \mathrm{~g} / \mathrm{mL})$, a vitamin stock solution $(40 \mathrm{mg}$ aminobenzoate, $10 \mathrm{mg}$ D-biotin, $30 \mathrm{mg}$ folate, $10 \mathrm{mg}$ lipoate, $100 \mathrm{mg}$ nicotinate, $50 \mathrm{mg}$ Ca-D-pantothenate, $100 \mathrm{mg}$ pyridoxamine hiydroxochloride, 100mg thiamine hydrochloride, 50mg vitamin B12 per L, filter sterilized), a trace metal stock solution $\left(2.86 \mathrm{~g} \mathrm{H}_{3} \mathrm{BO}_{3}, 1.81 \mathrm{~g} \mathrm{MnCl}_{2} * 4 \mathrm{H}_{2} \mathrm{O}, 0.24 \mathrm{~g} \mathrm{ZnSO} * 7 \mathrm{H}_{2} \mathrm{O}, 0.075 \mathrm{~g}\right.$ $\mathrm{CuSO} 4 * 5 \mathrm{H} 2 \mathrm{O}, 0.0494 \mathrm{~g} \mathrm{CaCl} 2 * 6 \mathrm{H}_{2} \mathrm{O}, 0.005 \mathrm{~g} \mathrm{NiCl} * 6 \mathrm{H}_{2} \mathrm{O}$ per $\mathrm{L}$ ), and $100 \mu \mathrm{L}$ of $1 \mathrm{mM}$ stock FeEDTA solution. 200mL cultures were grown in the Webb laboratory (courtesy of Chris Suffridge) and the cultures were centrifuged to remove cells, media was decanted into $50 \mathrm{~mL}$ polypropylene tubes and frozen. Prior to analysis, the samples were thawed and $25 \mathrm{~mL}$ of media was filtered $(0.2 \mu \mathrm{m}$, supor polyether sulfone sterivex) and extracted through $0.5 \mathrm{~g}$ C18 solid phase extraction columns (biotage), rinsed with $\mathrm{qH}_{2} \mathrm{O}$, and eluted with $5 \mathrm{~mL} \mathrm{MeOH}$. The extracts 
were concentrated under an $\mathrm{N}_{2}$ stream and analyzed by LC-ICPMS as described above for the Synechococcus sp. PCC 7002 culture.
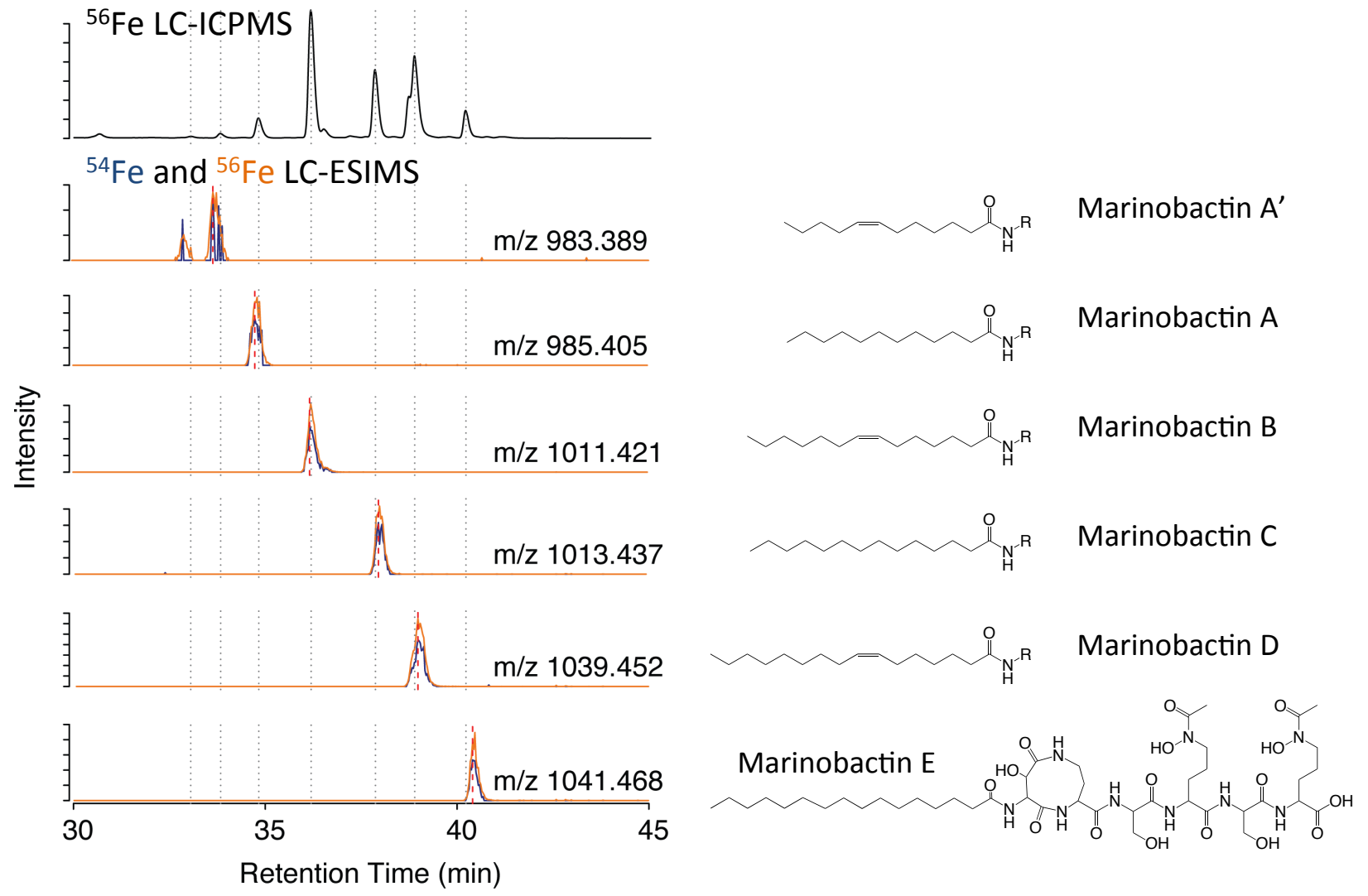

Figure A3.1 Marinobactins identified from alteromonas 2E5. The LC-ICPMS chromatogram shows seven distinct iron peaks, and the masses of each peak were determined using the isotope searching algorithms described above. Extracted ion chromatograms for the ${ }^{54} \mathrm{Fe}$ and ${ }^{56} \mathrm{Fe}$ isotopologues $\left[\mathrm{M}+\mathrm{Fe}^{3+}-2 \mathrm{H}^{+}\right]$are show, scaled by the natural abundance ratio of ${ }^{56} \mathrm{Fe} /{ }^{54} \mathrm{Fe}$ (divided by 15.7) so that the isotopologues overlap (left panel). Masses correspond to the $\left[\mathrm{M}+{ }^{56} \mathrm{Fe}^{3+}-2 \mathrm{H}^{+}\right]$isotopologues. Corresponding marinobactin structures are show to the right. The structure of Marinobactin A' was putatively identified based on exact mass. 


\title{
Chapter 4: Siderophore-based microbial adaptations to iron scarcity across the eastern Pacific
}

Authors: Rene M. Boiteau ${ }^{1,2}$, Nicholas J. Hawco, ${ }^{1,2}$, Daniel R. Mende ${ }^{3}$, Matthew R. McIlvin ${ }^{1}$, Peter N. Sedwick ${ }^{4}$, Mak A. Saito ${ }^{1}$, Edward F. Delong ${ }^{3}$, Daniel J. Repeta ${ }^{1}$

${ }^{1}$ Department of Marine Chemistry and Geochemistry, Woods Hole Oceanographic Institution, Woods Hole, MA.

${ }^{2}$ Department of Earth, Atmospheric, and Planetary Sciences, Massachusetts Institute of Technology, Cambridge, MA.

${ }^{3}$ Center for Microbial Oceanography Research and Education, University of Hawaii at Manoa, Honolulu, HI.

${ }^{4}$ Department of Ocean, Earth and Atmospheric Sciences, Old Dominion University, Norfolk, VA

\begin{abstract}
:
Iron availability regulates microbial growth and ecosystem productivity in vast regions of the ocean [Boyd et al., 2007; Moore et al., 2013]. Nearly all dissolved iron is complexed to strong organic ligands [Gledhill and Buck, 2012]. As competition for iron intensifies, microbes face enormous selective pressure to develop effective strategies to take up and utilize this organically bound iron [Dutkiewicz et al., 2009]. However, the composition of organic ligands in seawater and their control over iron bioavailability is poorly understood. Here we show that siderophores are a dynamic component of the ligand pool across the iron-starved eastern Pacific. We detected twenty-seven distinct ligands, eleven of which could be identified as known or novel siderophores. Siderophore concentrations were five-fold higher in iron-deficient offshore waters $(9 \mathrm{pM})$ compared with coastal and oligotrophic waters, and were dominated by amphibactins, amphiphilic siderophores that partition into cell membranes. Amphibactins were replaced with lower concentrations (1-2pM) of hydrophilic ferrioxamine siderophores in coastal and oligotrophic regions. A survey of global ocean metagenomes revealed that amphibactin synthesis genes are also present in other major iron-starved regions. Our results show that production and uptake of amphiphilic siderophores that can be tethered to cell membranes to reduce diffusive loss to the environment is likely a widespread adaptation of microbial communities in ironlimited regions of the ocean. These adaptations impact dissolved iron bioavailability and thus
\end{abstract}


have important consequences for marine ecosystem community structures and primary productivity.

\subsection{Main Text:}

Marine photosynthesis, respiration, and nitrogen fixation all depend on enzymes that require iron [Morel and Price, 2003], which is supplied to the ocean by continental weathering and deep sea hydrothermal activity[Boyd and Ellwood, 2010; Resing et al., 2015]. High biological demand in the sunlit portion of the marine water column, particularly in open ocean regions remote from continental inputs, can reduce dissolved iron ( $\mathrm{dFe}$ ) concentrations to $<0.1 \mathrm{nM}$ [Boyd and Ellwood, 2010]. These regions are often characterized by high nutrient, low chlorophyll (HNLC) conditions because iron scarcity limits phytoplankton growth and prevents complete macronutrient utilization [Boyd et al., 2007]. Effectively all marine dFe is complexed by organic ligands of unknown source or composition. These compounds keep otherwise insoluble Fe(III) in solution, but may also change its bioavailability by impeding iron transport into cells [Gledhill and Buck, 2012].

Electrochemical measurements have demonstrated that the strongest ligands in seawater have stability constants similar to siderophores, compounds exuded by microbes under iron stress to facilitate iron uptake [Gledhill and Buck, 2012]. Marine microbes synthesize a wide array of structurally diverse siderophores in laboratory cultures [Sandy and Butler, 2009] and metagenomic surveys hint that homologs to known siderophore uptake genes are common in iron-deficient ocean regions, even though siderophore biosynthesis genes themselves have not been found [Hopkinson and Barbeau, 2012; Toulza et al., 2012]. Siderophore production may be an important strategy to capture iron from the environment while preventing uptake by competitors. However, few siderophores have been directly observed in the environment [Mawji et al., 2008; Gledhill and Buck, 2012], and diffusive loss of siderophores makes them a potentially cost prohibitive means of iron acquisition [Völker and Wolf-Gladrow, 1999]. Alternative uptake mechanisms that reduce ligand-bound Fe(III) to bioavailable Fe(II) directly at the cell surface may be energetically favored in iron-poor HNLC regions, although their 
effectiveness also depends on the speciation and reactivity of exogenous iron [Shaked and Lis, 2012]. Insight into the viability of these different strategies lies within the iron-ligand pool.

Characterizing extremely low concentrations of iron binding ligands within the complex mixture of organic compounds dissolved in seawater poses a formidable analytical challenge. We developed an approach that combines trace metal clean reverse phase liquid chromatography with inductively coupled plasma mass spectrometry (LC-ICPMS) and electrospray ionization mass spectrometry (LC-ESIMS), to identify major iron ligands across the eastern tropical South Pacific Ocean. This is one of the most productive regions in the world's oceans, with high nutrient concentrations supplied by intense wind-driven upwelling along the coast (Fig. 4.1, coastal) [Bruland et al., 2005; Pennington et al., 2006]. As surface waters circulate westward, macronutrients and iron are drawn down by fast growing microplankton. Due to the low rates of atmospheric dust deposition in this region, dissolved iron is depleted relative to macronutrients [Hutchins et al., 2002; Bruland et al., 2005]. Chlorophyll levels drop, and the microbial community shifts to nanoplankton that can utilize the limited iron stocks more efficiently (Fig. 4.1, HNLC). Eventually, nitrate and phosphate are also depleted and nanoplankton are succeeded by cyanobacteria and picoeukaryotes that can effectively compete for these macronutrients (Fig. 4.1, oligotrophic). To investigate iron-binding ligands in these three ecosystems, we collected organic extracts from near-surface seawater during the 2013 US GEOTRACES Eastern Pacific Zonal Transect (EPZT) cruise (Fig. 4.1a).

Twenty-seven unique iron-containing compounds were detected by LC-ICPMS across the six surface water samples (Table S4.1, Fig. S4.1). Using LC-ESIMS we identified the major compound eluting at 22.9 min as ferrioxamine $\mathrm{B}$, an hydroxamate siderophore produced by both gram-positive and gram-negative bacteria including species of Actinomycetes and Vibrio (Fig. 4.2) [Martinez et al., 2001]. The chromatograms also showed low concentrations of nonpolar siderophores eluting between 35-50 minutes (Fig. 4.2). Many of these were identified as amphibactins, a suite of amphiphilic siderophores composed of a peptidic head group with a fatty acid side chain of variable structure. Amphibactins have been reported in marine Gammaproteobacteria, and are produced by two non-ribosomal peptide synthetases [Martinez et al., 2003; Vraspir et al., 2011]. Finally, two major iron-organic compounds with monoisotopic 
masses of 959.429, and 709.372 were also identified at 47.1 and 50.9 minutes respectively. These masses do not appear in a database of 367 known siderophores [Baars et al., 2014], suggesting that they are novel compounds. Tandem mass spectra $\left(\mathrm{MS}^{2}\right)$ of the $959.429 \mathrm{~m} / \mathrm{z}$ ion suggest a siderophore structurally related to amphibactins, containing the same iron-binding head group.

There was a clear trend in the abundance of different siderophores across the three nutrient regimes. Hydrophilic siderophores with retention times between 18-28 minutes were most abundant in the coastal sample. While LC-ICPMS detects compounds that are complexed with iron, electrochemical approaches measure uncomplexed (iron-free) ligands, and ligands that exchange iron rapidly (typically 2-12 hours). Concentrations of these ligands often exceed $\mathrm{dFe}$ concentrations in marine surface waters [Gledhill and Buck, 2012]. To detect the presence of uncomplexed siderophores, we titrated samples with iron to saturate all strong ligands. In the coastal upwelling sample, no changes in iron-bound siderophore concentration were observed (Fig. 4.2), indicating that siderophores in this region were fully complexed with iron, consistent with the high ambient dissolved iron concentrations (0.15-1.1 nM, Fig. 4.1c). The elevated baseline observed upon iron addition is indicative of a large excess of chromatographically unresolved organic compounds that can act as weak iron binding ligands (Fig. 4.2a). These structurally ill-defined compounds were found at similar concentrations across all samples and are likely refractory degradation products that comprise the majority of dissolved organic carbon in the ocean [Abdulla et al., 2010; Bundy et al., 2015; Repeta, 2015].

In the HNLC sample, the siderophore composition changed dramatically. Hydrophilic siderophores were less abundant, whereas amphiphilic siderophores and overall siderophore concentration were five-fold higher. This coincided with a decrease in dFe to $0.06-0.2 \mathrm{nM}$ (average $0.15 \mathrm{nM}$ ) and an increase in the abundance of uncomplexed siderophores, which here make up $40 \%$ of the total. Across the oligotrophic region, amphiphilic siderophore concentrations declined and hydrophilic siderophores returned. As with the HNLC regime, ambient dissolved iron concentrations are low $(0.04-0.13 \mathrm{nM})$ and a large fraction of siderophores (40-65\%) is not complexed with iron. Since dFe is higher than total siderophore concentration (3-9 $\mathrm{pM}$ ) in the HNLC and oligotrophic samples, the presence of a large fraction of iron-free 
siderophores is notable. One explanation is that most $\mathrm{dFe}$ is tightly complexed by other strong ligands that can compete with siderophores for iron [Gledhill and Buck, 2012]. Alternatively, the ligand exchange kinetics of $\mathrm{dFe}$ may be slow relative to the exudation rate of iron-free siderophores. As a result, a significant proportion of the total siderophores in these low iron regions is available to enhance the dissolution of iron from detrital particulate phases or atmospheric dust [Rue and Bruland, 1997; Akafia et al., 2014].

The changes that we observed in siderophore composition across marine biomes reflect how microbes acquire iron under different ecological conditions. In iron starved regions of the ocean, the intense selection pressure drives the microbial community to more effectively take up iron [Maldonado et al., 2001]. Increased concentrations of amphibactins (and siderophores overall) in these areas are indicative of positive selection pressure driving a phenotypic shift in iron acquisition strategy. Thus, the ability to produce these compounds and to utilize iron bound to them confers a specific competitive advantage to microbes inhabiting the HNLC Eastern tropical pacific, which must compete for scarce iron resources.

Differences in membrane affinity may explain why amphiphilic siderophores appear to be better adapted to iron-deficient niches compared to the more hydrophilic compounds. The lipid sidechain of amphibactins can partition into cell membranes [Martinez et al., 2003]. This may reduce the risk of losing a metabolically expensive compound to diffusive loss in environments where iron is scarce. The prevalence of more hydrophilic siderophores in other regions of the ocean implies that the amphiphilic strategy comes at a cost - potentially linked to the weaker binding strength of peptidic amphiphilic siderophores [Barbeau et al., 2001] (unpublished data) compared to ferrioxamines [Rue and Bruland, 1995]. Siderophores also participate in a wide range of microbial interactions related to iron acquisition, including inhibition of competitors, symbioses and pirating, and chemical signaling [Amin et al., 2009; Sandy and Butler, 2009]. It is therefore likely that the siderophores detected across these gradients have important consequences for members of the microbial community beyond their producers by facilitating iron uptake to some organisms while inhibiting it to others. 
Up to $90 \%$ of $\mathrm{dFe}$ uptake in iron-stressed regions is regenerated from the biotic pool by grazing and viral lysis of cells [Boyd et al., 2015]. Copiotrophic bacteria that feed on these lysates are a likely source of siderophores, which may provide an effective way for microbes to capture regenerated iron before it is scavenged by sinking particles. Indeed, the production of ferrioxamines and amphibactins by mixed marine bacterial communities is stimulated by amendments of glucose, glycerol, and chitin [Mawji et al., 2008, 2011]. Siderophores may also provide a source of bioavailable iron for phytoplankton expressing compatible uptake transporters acquired through horizontal gene transfer [Malmstrom et al., 2013]. Assuming a cellular iron to phosphorus (Fe:P) quota of 2-8mmol/mol [Twining et al., 2011] and Redfield cellular N:P (16:1), we estimate that microbes require $\sim 5-25 \mathrm{pM}$ Fe per day to sustain the $\mathrm{N}$ uptake rate of $\sim 50 \mathrm{nM}$ per day characteristic of the eastern tropical South Pacific HNLC region [Dugdale and Wilkerson, 1991]. At 9pM total siderophore concentrations, daily cycling could transfer regenerated iron into siderophores and support much of the microbial community iron requirement.

To complement our chemical analyses, we investigated the prevalence of amphibactin biosynthesis genes within ocean metagenomes. Although genes encoding siderophore uptake proteins are common in HNLC metagenomes, genes encoding siderophore biosynthesis have yet to be reported [Hopkinson and Barbeau, 2012; Toulza et al., 2012]. A pair of nonribosomal peptide synthetases (NRPS), ABO_2093 and ABO_2092, were recently implicated in the production of amphibactins by the Gammaproteobacteria Alcanivorax borkumensis [Kem et al., 2014]. We identified similar NRPS genes in the genomes of numerous marine Vibrio, and confirmed amphibactin production by five additional NRPS sequence pairs through laboratory culture experiments (supplementary information). Using a strict similarity cutoff ( $>90 \%$ identity) we searched for these six amphibactin NRPS pairs within the Tara Ocean metagenomic catalogue [Sunagawa et al., 2015]. Homologs to both amphibactin biosynthesis genes were found along the upwelling regions near California and Chile where we measured amphibactins in this study (Fig 4.3, Figs. S4.7-8, Chapter 5). Amphibactin production genes were also present in the South Atlantic - another region where iron limitation has been observed [Moore et al., 2013] and where amphibactin production has been documented in nutrient enrichment experiments [Mawji et al., 2011]. Despite spatial and temporal differences in these data sets, there is broad 
agreement between the regions where amphibactins and amphibactin synthesis genes were detected. Identifying these sequences in assembled genomes from HNLC environments will be the key to explicitly determine which organisms are responsible for producing amphibactins in the ocean.

The changes in siderophore distributions and concentrations we measured across the eastern Pacific Ocean provide strong evidence that siderophores are an active and important component of microbial iron cycling. The ability to produce and assimilate siderophores likely contributes to the competitive success of microbes that inhabit HNLC regions. Amphibactins dominated the siderophore distributions in HNLC areas of both the Eastern Tropical Pacific and CCS (Chapter 5). However, many different amphiphilic siderophores have been identified in marine microbes [Sandy and Butler, 2009], and further surveys of other iron starved regions could reveal a broader suite of siderophores produced under different environmental conditions. Amphiphilic siderophores can be tethered to cell membranes [Martinez et al., 2003], raising questions about the energetic trade-offs that select for specific siderophores, the partitioning of siderophores between dissolved and particulate phases, and the availability of siderophores to the microbial community at large. Contrary to current biogeochemical models that assume a single ligand class [Dutkiewicz et al., 2009], our results show major differences in siderophore composition across major biomes. These compounds potentially affect rates of iron regeneration, dissolution, scavenging, and uptake. Unraveling the cycling of siderophores through combined chemical, genomic, and laboratory culture approaches is therefore an important element to understanding productivity in the iron-starved ocean.

\subsection{Methods:}

\subsubsection{Materials and reagents:}

Ultra high purity water (18.2 $\left.\mathrm{MO} \mathrm{cm}, \mathrm{qH}_{2} \mathrm{O}\right)$ and LCMS grade methanol $(\mathrm{MeOH})$, ammonium formate, and formic acid (Optima, Fisher scientific) were used throughout this study. Methanol was purified to reduce $\mathrm{Fe}$ contamination by sub-boiling-point distillation in a polytetrafluoroethylene (PTFE) still. PTFE solid phase extraction (SPE) column hardware, and 
sample vials were soaked overnight in $0.1 \%$ detergent (Citranox), rinsed with $\mathrm{qH}_{2} \mathrm{O}$, and soaked for 1-2 days in $1 \mathrm{~N} \mathrm{HCl}$ (trace metal grade, Fisher Scientific), followed by a final $\mathrm{qH}_{2} \mathrm{O}$ rinse. Quartz evaporation flasks were combusted at $450^{\circ} \mathrm{C}$ and soaked in $1 \mathrm{~N} \mathrm{HCl}$. PTFE tubing and polyethersulfone capsule filters $(0.2 \mu \mathrm{m}$ Millipore) used for sample collection were flushed thoroughly with $1 \% \mathrm{HCl}$ and $\mathrm{qH}_{2} \mathrm{O}$ prior to use. Stock solutions of $50 \mu \mathrm{M}$ ferrioxamine and cyanocobalamin (Sigma Aldrich) were prepared in $\mathrm{qH}_{2} \mathrm{O}$. An iron citrate stock solution was prepared as $1 \mathrm{mM}$ sodium citrate dihydrate and $100 \mathrm{mM}$ iron (diluted from a $1000 \mathrm{ppm}$ iron reference standard solution, Fisher chemical) in $\mathrm{qH}_{2} \mathrm{O}$. For the isotope validation experiments, a $2000 \mathrm{ppm}{ }^{57} \mathrm{Fe}$ enriched solution was prepared by dissolving ${ }^{57} \mathrm{Fe}$ oxide (Cambridge Isotope Laboratories) in concentrated $\mathrm{HCl}$. This was diluted in a $1 \mathrm{mM}$ sodium citrate solution to make a $4 \mu \mathrm{M}{ }^{57} \mathrm{Fe}$ stock solution.

\subsubsection{Sample collection and processing:}

The sample collection method used in this study was designed to minimize metal contamination and sample processing (e.g. $\mathrm{pH}$ changes) to preserve the iron-organic compounds intact. Tracemetal clean filtered seawater was pumped by a tow-fish from $3 \mathrm{~m}$ depth while transiting across the cruise track during the US GEOTRACES EPZT cruise (Table S4.2). Thus, each sample represents an integrated average signal across a wide region. Between 400-600L of seawater was filtered continuously at a flow rate of $250 \mathrm{~mL} / \mathrm{min}$ and extracted through custom-made SPE columns packed with 20g ENV resin (Bondesil, Agilent). Immediately prior to sample collection, SPE columns were primed with distilled methanol, flushed with $\mathrm{qH}_{2} \mathrm{O}$, acidified to $\mathrm{pH} 2$ with dilute $\mathrm{HCl}$, and finally rinsed with $\mathrm{qH}_{2} \mathrm{O}$. Samples were stored at $-20^{\circ} \mathrm{C}$ and returned to the laboratory for further analyses. Thawed SPE columns were rinsed with $500 \mathrm{~mL}$ of $\mathrm{qH}_{2} \mathrm{O}$, and organic ligands were eluted with $250 \mathrm{~mL}$ of $\mathrm{MeOH}$. Extracts were concentrated by rotary evaporation and the final volume was adjusted to $6 \mathrm{~mL}$ with $\mathrm{qH}_{2} \mathrm{O}$. Samples were stored at $-20^{\circ} \mathrm{C}$ in PTFE vials. Aliquots $(1 \mathrm{~mL})$ of each concentrated sample were removed and spiked with $20 \mu \mathrm{L}$ of $50 \mu \mathrm{M}$ cyanocobalamin (Sigma Aldrich) as an internal standard. A sample blank was also collected by pumping only $200 \mathrm{~mL}$ of filtered seawater through an SPE column, which was frozen, processed, and analyzed with the six seawater samples. Nitrate, dissolved iron, and 
pigment samples were collected and analyzed as described previously [Hooker et al., 2005; Hydes et al., 2010; Resing et al., 2015].

\subsubsection{Liquid chromatography:}

Organic extracts were separated on an Agilent 1260 series bioinert high pressure liquid chromatography (HPLC) system fitted with a C8 column (Hamilton, $2.1 \times 100 \mathrm{~mm}, 3 \mu \mathrm{m}$ particle size) and polyetheretherketone (PEEK) tubing and connectors. Ligands were eluted with (A) $5 \mathrm{mM}$ aqueous ammonium formate and (B) $5 \mathrm{mM}$ ammonium formate in distilled $\mathrm{MeOH}$ using a 50 minute gradient from $10-90 \% \mathrm{~B}$, followed by isocratic elution at $90 \% \mathrm{~B}$ for 10 minutes at a flow rate of $0.2 \mathrm{~mL} / \mathrm{min}$. A post column PEEK flow splitter directed $50 \mu \mathrm{L} / \mathrm{min}$ into the ICPMS or ESIMS.

\subsubsection{Inductively coupled plasma mass spectrometry (ICPMS):}

The method for LC-ICPMS analysis was adapted from Boiteau et al. (2013) [Boiteau et al., 2013]. The flow of the LC column was coupled directly to a quadrupole ICPMS (iCAP Q, Thermo Scientific) using a perfluoroalkoxy micronebulizer (PFA-ST, Elemental Scientific) and a cyclonic spray chamber cooled to $0^{\circ} \mathrm{C}$. Oxygen gas was introduced to the plasma at $25 \mathrm{~mL} / \mathrm{min}$ to prevent the deposition of reduced organics on the cones. The ICPMS was equipped with platinum sampler and skimmer cones. ${ }^{56} \mathrm{Fe},{ }^{57} \mathrm{Fe}$, and ${ }^{59} \mathrm{Co}$ were monitored with an integration time of 0.05 seconds each. Measurements were made in kinetic energy discrimination mode with a He collision gas introduced at a rate of $4.2 \mathrm{~mL} / \mathrm{min}$ to remove $\mathrm{ArO}^{+}$interferences on ${ }^{56} \mathrm{Fe}$.

ICPMS signals were exported into $\mathrm{R}$ for data processing. The chromatographic baseline was subtracted using an asymmetric least squares algorithm through the 'baseline' $r$ package. Chromatographic peaks were quantified as two groups: hydrophilic compounds (retention time of 18-28 minutes), and amphiphilic compounds (retention time of 35-55 minutes). Peak areas were integrated and used to calculate concentrations with a six point calibration curve of a ferrioxamine $\mathrm{E}$ standard solution (retention time $=19.8 \mathrm{~min}$ ) with concentrations ranging from $3 \mathrm{nM}$ to $100 \mathrm{nM}$. Signals were standardized between samples based on the ${ }^{59} \mathrm{Co}$ peak area of the 
cyanocobalamin internal standard to correct for instrument drift and matrix effects on ICPMS sensitivity (these corrections were $<10 \%$ ). No naturally occurring cyanocobalamin was detected in the samples. Since only the iron-bound form is quantified by LC-ICPMS, samples were titrated with excess iron citrate and re-analyzed to quantify total siderophore concentrations. A 1:10 addition of the iron citrate stock solution was sufficient to saturate the unbound iron complexes. Our reported concentrations of amphiphilic compounds eluting after 19.8 minutes were not corrected for the decrease in LC-ICPMS signal intensity with increased organic solvent load (a $25 \%$ decrease was observed in $100 \%$ organic solvent compared to $50 \%$ ) and are therefore conservative. Analytical uncertainty was determined by 4-5 replicate analyses for two samples (standard deviation of $9.1 \%$ and $9.6 \%$ for hydrophilic and amphiphilic siderophore concentrations respectively).

\subsubsection{Electrospray ionization mass spectrometry (ESIMS) analysis:}

For determination of the siderophore mass, the flow from the LC was coupled to an Orbitrap Fusion mass spectrometer (Thermo Scientific) equipped with a heated electrospray ionization source. ESI source parameters were set to a capillary voltage of $3500 \mathrm{~V}$, sheath, auxiliary and sweep gas flow rates of 12, 6, and 2 (arbitrary units), and ion transfer tube and vaporizer temperatures of $300^{\circ} \mathrm{C}$ and $75^{\circ} \mathrm{C}$. MS1 scans were collected in high resolution $(450 \mathrm{~K})$ positive mode. High energy collision induced dissociation (HCD) MS2 spectra were collected on the ion trap mass analyzer. Ions were trapped using a quadrupole isolation window of $1 \mathrm{~m} / \mathrm{z}$ and were then fragmented using an HCD collision energy of 35\%.

The LC-ESIMS data was converted to mzXML file format (MSconvert, proteowizard) and a constant time offset was applied to align the retention time of the cyanocobalamin $[\mathrm{M}+\mathrm{H}]^{2+}$ peak $(\mathrm{m} / \mathrm{z}=678)$ with the cyanocobalamin ${ }^{59} \mathrm{Co}$ peak in the LC-ICPMS chromatogram. Using an isotope pattern search algorithm described previously [Boiteau and Repeta, 2015], candidate masses were determined for the iron-bound form of many of the detected compounds by identifying $\mathrm{m} / \mathrm{z}$ and intensity features from each scan that match mass difference and abundance

ratio of ${ }^{56} \mathrm{Fe}$ and ${ }^{54} \mathrm{Fe}$, and that appear as coherent peaks that match the retention time of the LCICPMS peaks (Figs. S4.2-S4.5). In some cases, the apo (iron free) mass was also observed. 


\subsubsection{Validation by ${ }^{57} \mathrm{Fe}$ spike:}

To validate masses as features that contain iron, rather than coeluting ions that coincidentally match the iron isotope pattern, isotopically enriched ${ }^{57} \mathrm{Fe}$ was added to samples to produce an observable isotopic shift in the $\mathrm{m} / \mathrm{z}$ ratio of the iron-bound siderophore complex. Aliquots of the organic extracts were spiked with ${ }^{57} \mathrm{Fe}$ and analyzed by LC-ESIMS in parallel to the ${ }^{56} \mathrm{Fe}$ addition experiments (described above). Features that contain iron show an increase in the

intensity ratio of the ${ }^{57} \mathrm{Fe}$ isotopologue compared to the ${ }^{56} \mathrm{Fe}$ isotopologue in the ${ }^{57} \mathrm{Fe}$ enriched treatment (Fig. S4.6). $\mathrm{MS}^{2}$ fragmentation patterns were obtained for validated masses (Figs. $\mathrm{S} 4.2 \mathrm{c}-\mathrm{S} 4.5 \mathrm{c}$ ). Only uncomplexed siderophores were labeled with ${ }^{57} \mathrm{Fe}$ during these 1-4 hour long experiments, indicating that isotopic exchange of siderophore-bound iron is slow.

\subsubsection{Siderophore identification:}

The identities of the detected iron ligands were determined by first comparing their masses to those in a library of 367 known siderophores based on the Chelomex siderophore database[Baars et al., 2014]. The masses of peak $\mathrm{g}$ and peaks o, $\mathrm{q}, \mathrm{r}, \mathrm{u}, \mathrm{w}, \mathrm{x}, \mathrm{y}$, and $\mathrm{z}$ matched those of ferrioxamine B and amphibactins (Table S4.1, Figs. S4.2,S4.3). Although background noise at the mass and retention time of ${ }^{57} \mathrm{Fe}$-ferrioxamine $\mathrm{B}$ interfered with validating the $614 \mathrm{~m} / \mathrm{z}$ feature by isotope addition, the retention time and $\mathrm{MS}^{2}$ fragmentation pattern matched that of an authentic Ferrioxamine B standard. Ferrioxamine B has distinctive major fragments of 414.2 and $496.1 \mathrm{~m} / \mathrm{z}$, corresponding to peptide bond cleavages and losses of $\mathrm{C}_{9} \mathrm{H}_{16} \mathrm{~N}_{2} \mathrm{O}_{3}$ and $\mathrm{C}_{5} \mathrm{H}_{14} \mathrm{~N}_{2} \mathrm{O}$ [Gledhill, 2001]. Similarly, we confirmed the identities of peaks o, q, r, u, w, x, y, and $\mathrm{z}$ as amphibactins by comparing their fragmentation spectra to authentic compounds isolated from Vibrio cyclitrophicus FF75. The $\mathrm{MS}^{2}$ fragmentation spectra of these compounds show diagnostic cleavages along the peptidic bonds, resulting in neutral losses of $\mathrm{m} / \mathrm{z} 218$ $\left(\mathrm{C}_{8} \mathrm{H}_{14} \mathrm{~N}_{2} \mathrm{O}_{5}\right), 277\left(\mathrm{C}_{10} \mathrm{H}_{19} \mathrm{~N}_{3} \mathrm{O}_{6}\right)$, and $305\left(\mathrm{C}_{11} \mathrm{H}_{19} \mathrm{~N}_{3} \mathrm{O}_{7}\right)$ as well as head group fragment ions of $\mathrm{m} / \mathrm{z} 485,371$, and 112 that lack the fatty acid side chain [Martinez et al., 2003; Vraspir et al., 2011]. 
The masses of the compound eluting at 46.8 and 50.9 minutes (peaks $\mathrm{v}$ and aa, $959.43 \mathrm{~m} / \mathrm{z}$ and $709.372 \mathrm{~m} / \mathrm{z}$ moniosotopic masses, Figs. S4.4,S4.5) were both validated by ${ }^{57} \mathrm{Fe}$ addition, but did not match any known compound in the database. The $959.43 \mathrm{~m} / \mathrm{z}$ compound has the same major fragments listed above for the amphibactins, indicating that it contains the same peptidic ironbinding head group composed of three N-hydroxylated and acetylated ornithine residues and one serine residue. The $709.372 \mathrm{~m} / \mathrm{z}$ compound is structurally distinct, exhibiting major neutral losses that include $61,115,132,276$, and $316 \mathrm{~m} / \mathrm{z}$.

\subsubsection{Vibrio laboratory culture:}

Homologs to the two known amphibactin biosynthesis NRPSs from Alkanivorax borkumensis SK2 (ABO_2092 and ABO_2093) with $>40 \%$ sequence similarity were found in the National Center for Biotechnology Information database using blastp. Five protein pairs were selected from cultured Vibrio species for further investigation. Using the PKS/NRPS analysis webserver from the University of Maryland [Bachmann and Ravel, 2009], these genes were predicted to assemble (from $\mathrm{N}$ to $\mathrm{C}$ terminus) $\mathrm{N}^{5}$-acyl- $\mathrm{N}^{5}$-hydroxyornithine - $\mathrm{N}^{5}$-acyl- $\mathrm{N}^{5}$-hydroxyornithine serine - $\mathrm{N}^{5}$-acyl- $\mathrm{N}^{5}$-hydroxyornithine, which is consistent with the production of amphibactin. These strains (Vibrio cyclitrophicus FF75, Vibrio cyclitrophicus 1F-53, Vibrio splendidus ZS139, Vibrio Splendidus ZF-90, and Vibrio Lentus ZS-17, generously provided by the Polz group at the Massachusetts Institute of Technology) were grown in low iron casamino acid based media as described previously [Martinez et al., 2003; Vraspir et al., 2011]. Amphibactins were extracted from $10 \mathrm{~mL}$ of filtered media onto ENV SPE columns, eluted with methanol, and detected by LC-ESIMS. The presence of amphibactins from these iron-starved culture confirmed that these gene sequences are responsible for amphibactin production, and provided additional query sequences for the detection of these genes from metagenomes.

\subsubsection{Tara Ocean catalog search:}

In order to detect genes from the Tara Ocean Microbial Reference Catalog (OMRGC)[Sunagawa et al., 2015] that correspond to the amphibactin biosynthesis genes from Alkanivorax 
borkumensis or the Vibrio strains from this study, we used tblastn[Altschul et al., 1990]. Genes in the Tara OMRGC with at least $90 \%$ amino acid identity to the amphibactin biosynthesis genes were considered of same functionality (Fig. S4.7). The global abundance profile of these genes was then extracted from the Tara OMRGC. The genes were deemed present if they had a combined coverage of at least $1 \mathrm{X}$ within a sample. Both genes were found in samples collected from four stations $(133,93,94$, and 70$)$ in late summer to early fall. Fig. S4.8 visualizes the global distribution of the selected amphibactin biosynthesis genes across depths, and Fig. 4.3 in the main text shows the combined results irrespective of depth.

\subsection{Acknowledgments:}

We would like to thank B Twining, and B Bidigare for pigment community data, the Ocean Data Facility for nitrate measurements, NASA Ocean Biology (OB.DAAC) for SeaWiFS sea surface chlorophyll data, and P Arevalo, M Cutler and M Polz for providing Vibrio strains. We are grateful to co-chief scientists J Moffett and C German, G Smith for sampling assistance, B Sohst for iron data, and the scientists and crew of the US GEOTRACES EPZT cruise. Support was provided by the National Science Foundation (NSF) program in Chemical Oceanography (OCE1356747, OCE-1233261, and OCE-1237034), the NSF Science and Technology Center for

Microbial Oceanography Research and Education (C-MORE; DBI-0424599), the Gordon and Betty Moore Foundation, and the Simons Foundation.

\subsection{References:}

Abdulla, H. A. N., E. C. Minor, R. F. Dias, and P. G. Hatcher (2010), Changes in the compound classes of dissolved organic matter along an estuarine transect: A study using FTIR and 13C NMR, Geochim. Cosmochim. Acta, 74(13), 3815-3838, doi:10.1016/j.gca.2010.04.006.

Akafia, M. M., J. M. Harrington, J. R. Bargar, and O. W. Duckworth (2014), Metal oxyhydroxide dissolution as promoted by structurally diverse siderophores and oxalate, Geochim. Cosmochim. Acta, 141, 258-269, doi:10.1016/j.gca.2014.06.024.

Altschul, S. F., W. Gish, W. Miller, E. W. Myers, and D. J. Lipman (1990), Basic Local Alignment Search Tool, J Mol Biol., 215(3), 403-410.

Amin, S. A., D. H. Green, M. C. Hart, F. C. Kupper, W. G. Sunda, and C. J. Carrano (2009), Photolysis of iron-siderophore chelates promotes bacterial-algal mutualism, Proc. Natl. 
Acad. Sci., 106(40), 17071.

Baars, O., F. M. M. Morel, and D. H. Perlman (2014), ChelomEx: Isotope-Assisted Discovery of Metal Chelates in Complex Media Using High-Resolution LC-MS, Anal. Chem., 86(22), 11298-11305, doi:10.1021/ac503000e.

Bachmann, B. O., and J. Ravel (2009), In Silico Prediction of Microbial Polyketide and Nonribosomal Peptide Biosynthetic Pathways from DNA Sequence Data, Methods Enzymol., 458(09), 181-217, doi:10.1016/S0076-6879(09)04808-3.

Barbeau, K. A., E. L. Rue, K. W. Bruland, and A. Butler (2001), Photochemical cycling of iron in the surface ocean mediated by microbial iron(III)-binding ligands., Nature, 413(6854), 409-13, doi:10.1038/35096545.

Boiteau, R. M., and D. J. Repeta (2015), An extended siderophore suite from Synechococcus sp. PCC 7002 revealed by LC-ICPMS-ESIMS, Metallomics, 7(5), 877-884, doi:10.1039/C5MT00005J.

Boiteau, R. M., J. N. Fitzsimmons, D. J. Repeta, and E. A. Boyle (2013), Detection of iron ligands in seawater and marine cyanobacteria cultures by high-performance liquid chromatography-inductively coupled plasma-mass spectrometry., Anal. Chem., 85(9), 4357-62, doi:10.1021/ac3034568.

Boyd, P. W., and M. J. Ellwood (2010), The biogeochemical cycle of iron in the ocean, Nat. Geosci., 3(10), 675-682, doi:10.1038/ngeo964.

Boyd, P. W. et al. (2007), Mesoscale Iron Enrichment Experiments 1993-2005: Synthesis and Future Directions, Science, 315(February), 612-617.

Boyd, P. W., R. F. Strzepek, M. J. Ellwood, D. A. Hutchins, S. D. Nodder, B. S. Twining, and S. W. Wilhelm (2015), Why are biotic iron pools uniform across high- and low-iron pelagic ecosystems?, Global Biogeochem. Cycles, 29(7), 1028-1043, doi:10.1002/2014GB005014.

Bruland, K. W., E. L. Rue, G. J. Smith, and G. R. DiTullio (2005), Iron, macronutrients and diatom blooms in the Peru upwelling regime: brown and blue waters of Peru, Mar. Chem., 93(2-4), 81-103, doi:10.1016/j.marchem.2004.06.011.

Bundy, R. M., H. A. N. Abdulla, P. G. Hatcher, D. V. Biller, K. N. Buck, and K. a. Barbeau (2015), Iron-binding ligands and humic substances in the San Francisco Bay estuary and estuarine-influenced shelf regions of coastal California, Mar. Chem., 173, 183-194, doi:10.1016/j.marchem.2014.11.005.

Dugdale, R. C., and F. P. Wilkerson (1991), Low specific nitrate uptake rate : A common feature of marine ecosystems, Limnol. Oceanogr., 36(8), 1678-1688.

Dutkiewicz, S., M. J. Follows, and J. G. Bragg (2009), Modeling the coupling of ocean ecology 
and biogeochemistry, Global Biogeochem. Cycles, 23(4), 1-15, doi:10.1029/2008GB003405.

Gledhill, M. (2001), Electrospray ionisation-mass spectrometry of hydroxamate siderophores, Analyst, 126(8), 1359-1362, doi:10.1039/b1012681.

Gledhill, M., and K. N. Buck (2012), The organic complexation of iron in the marine environment: a review., Front. Microbiol., 3, 1-17, doi:10.3389/fmicb.2012.00069.

Hooker, S. B. et al. (2005), The Fourth SeaWiFS HPLC Analysis Round-Robin Experiment (SeaHARRE-4), NASA Tech. Memo, 2005-21278, 112pp.

Hopkinson, B. M., and K. A. Barbeau (2012), Iron transporters in marine prokaryotic genomes and metagenomes., Environ. Microbiol., 14(1), 114-28, doi:10.1111/j.14622920.2011.02539.x.

Hutchins, D. A., C. E. Hare, R. S. Weaver, Y. Zhang, G. F. Firme, G. R. DiTullio, M. B. Alm, S. F. Riseman, J. M. Maucher, and M. E. Geesey (2002), Phytoplankton iron limitation in the Humboldt Current and Peru Upwelling, Limnol. Oceanogr., 47(4), 997-1011, doi:10.4319/lo.2002.47.4.0997.

Hydes, D. et al. (2010), Determination of dissolved nutrients (N, P, Si) in seawater with high precision and inter-comparability using gas-segmented continuous flow analysers, Go-sh. Repeat Hydrogr. Man. IOCCP Rep., 134(14), 1-87.

Kem, M. P., H. K. Zane, S. D. Springer, J. M. Gauglitz, and A. Butler (2014), Amphiphilic siderophore production by oil-associating microbes, Metallomics, 6(6), 1150, doi:10.1039/c4mt00047a.

Maldonado, M. T., P. W. Boyd, J. LaRoche, R. Strzepek, A. Waite, A. R. Bowie, P. L. Croot, R. D. Frew, and N. M. Price (2001), Iron uptake and physiological response of phytoplankton during a mesoscale Southern Ocean iron enrichment, Limnol. Oceanogr., 46(7), 1802-1808, doi:10.4319/lo.2001.46.7.1802.

Malmstrom, R. R., S. Rodrigue, K. H. Huang, L. Kelly, S. E. Kern, A. Thompson, S. Roggensack, P. M. Berube, M. R. Henn, and S. W. Chisholm (2013), Ecology of uncultured Prochlorococcus clades revealed through single-cell genomics and biogeographic analysis., ISME J., 7, 184-198, doi:10.1038/ismej.2012.89.

Martinez, J. S., M. G. Haygood, and A. Butler (2001), Identification of a natural desferrioxamine siderophore produced by a marine bacterium, Limnol. Oceanogr., 46(2), 420-424, doi:10.4319/1o.2001.46.2.0420.

Martinez, J. S., J. N. Carter-Franklin, E. L. Mann, J. D. Martin, M. G. Haygood, and A. Butler (2003), Structure and membrane affinity of a suite of amphiphilic siderophores produced by 
a marine bacterium., Proc. Natl. Acad. Sci. U. S. A., 100(7), 3754-9, doi:10.1073/pnas.0637444100.

Mawji, E., M. Gledhill, J. A. Milton, G. A. Tarran, S. Ussher, A. Thompson, G. A. Wolff, P. J. Worsfold, and E. P. Achterberg (2008), Hydroxamate siderophores: occurrence and importance in the Atlantic Ocean., Environ. Sci. Technol., 42(23), 8675-80.

Mawji, E., M. Gledhill, J. A. Milton, M. V. Zubkov, A. Thompson, G. A. Wolff, and E. P. Achterberg (2011), Production of siderophore type chelates in Atlantic Ocean waters enriched with different carbon and nitrogen sources, Mar. Chem., 124(1-4), 90-99, doi:10.1016/j.marchem.2010.12.005.

Moore, C. M. et al. (2013), Processes and patterns of oceanic nutrient limitation, Nat. Geosci., 6(9), 701-710, doi:10.1038/ngeo1765.

Morel, F. M. M., and N. M. Price (2003), The biogeochemical cycles of trace metals in the oceans., Science, 300(5621), 944-7, doi:10.1126/science.1083545.

Pennington, J. T., K. L. Mahoney, V. S. Kuwahara, D. D. Kolber, R. Calienes, and F. P. Chavez (2006), Primary production in the eastern tropical Pacific: A review, Prog. Oceanogr., 69(2-4), 285-317, doi:10.1016/j.pocean.2006.03.012.

Repeta, D. J. (2015), Chemical characterization and cycling of dissolved organic matter, in Biogeochemistry of Marine Dissolved Organic Matter, edited by D. A. Hansell and C. A. Carlson, Elsevier.

Resing, J. A., P. N. Sedwick, C. R. German, W. J. Jenkins, J. W. Moffett, B. M. Sohst, and A. Tagliabue (2015), Basin-scale transport of hydrothermal dissolved metals across the South Pacific Ocean, Nature, 523(7559), 200-203, doi:10.1038/nature14577.

Rue, E., and K. Bruland (1995), Complexation of iron(III) by natural organic ligands in the Central North Pacific as determined by a new competitive ligand equilibration/adsorptive cathodic stripping voltammetric method, Mar. Chem., 50(1-4), 117-138, doi:10.1016/03044203(95)00031-L.

Rue, E. L., and K. W. Bruland (1997), The role of organic complexation on ambient iron chemistry in the equatorial Pacific Ocean and the response of a mesoscale iron addition experiment, Limnol. Oceanogr., 42(5), 901-910, doi:10.4319/1o.1997.42.5.0901.

Sandy, M., and A. Butler (2009), Microbial iron acquisition: marine and terrestrial siderophores., Chem. Rev., 109(10), 4580-95, doi:10.1021/cr9002787.

Shaked, Y., and H. Lis (2012), Disassembling iron availability to phytoplankton., Front. Microbiol., 3(April), 123, doi:10.3389/fmicb.2012.00123.

Sunagawa, S. et al. (2015), Structure and function of the global ocean microbiome, Science, 
348(6237), 1261359-1261359, doi:10.1126/science.1261359.

Toulza, E., A. Tagliabue, S. Blain, and G. Piganeau (2012), Analysis of the global ocean sampling (GOS) project for trends in iron uptake by surface ocean microbes., PLoS One, 7(2), e30931, doi:10.1371/journal.pone.0030931.

Twining, B. S., S. B. Baines, J. B. Bozard, S. Vogt, E. a. Walker, and D. M. Nelson (2011), Metal quotas of plankton in the equatorial Pacific Ocean, Deep. Res. Part II Top. Stud. Oceanogr., 58(3-4), 325-341, doi:10.1016/j.dsr2.2010.08.018.

Völker, C., and D. A. Wolf-Gladrow (1999), Physical limits on iron uptake mediated by siderophores or surface reductases, Mar. Chem., 65(3-4), 227-244, doi:10.1016/S03044203(99)00004-3.

Vraspir, J. M., P. D. Holt, and A. Butler (2011), Identification of new members within suites of amphiphilic marine siderophores., Biometals, 24(1), 85-92, doi:10.1007/s10534-010-93781 . 


\subsection{Figures:}
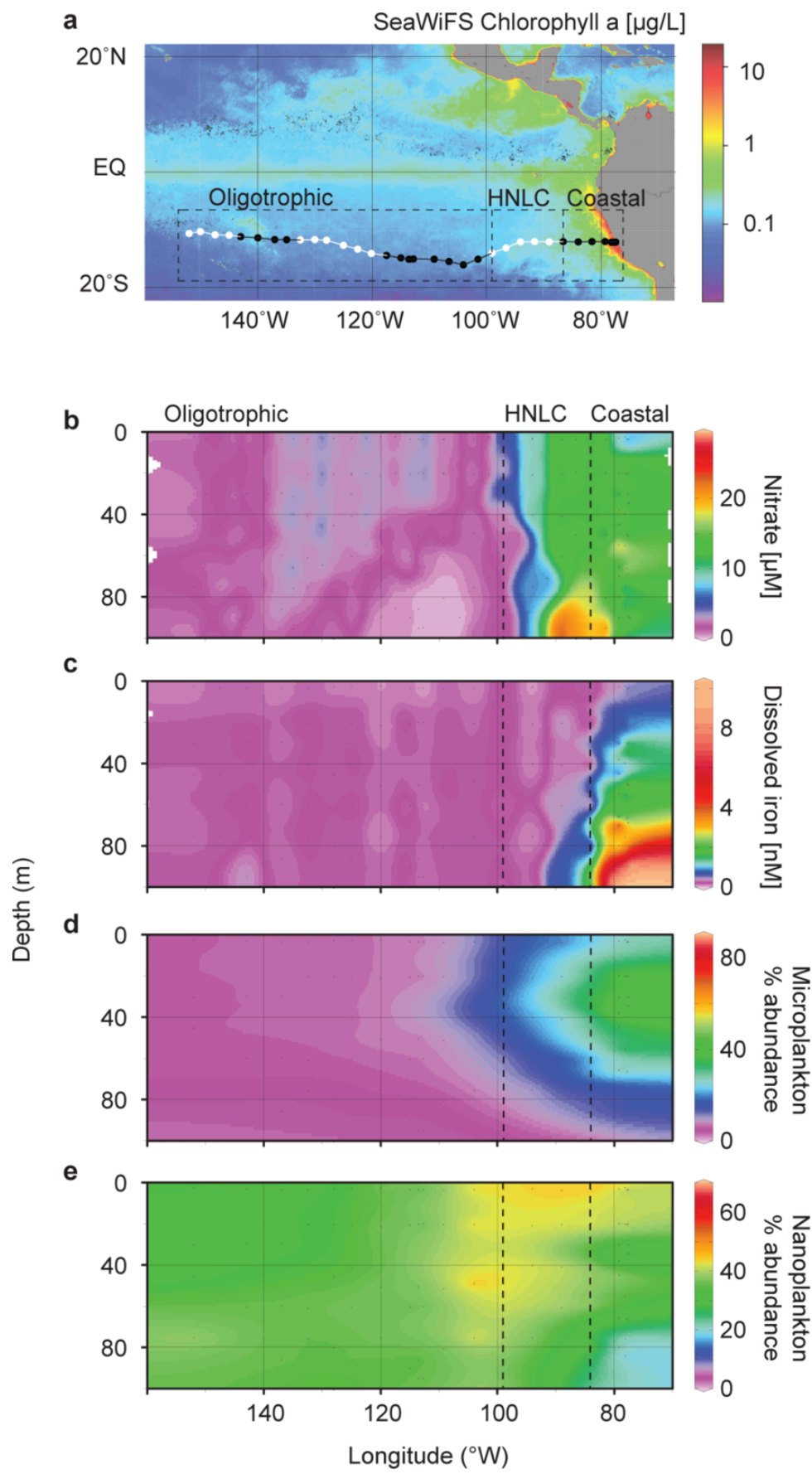

Figure 4.1: (a) US GEOTRACES EPZT cruise track map with SeaWiFS-derived average sea surface chlorophyll (austral summer 2013-2014). Surface seawater was collected continuously from a trace metal clean tow fish across coastal (sample 1), high nutrient low chlorophyll (sample 2), and oligotrophic (samples 3-6) regions. Alternating black and white lines represent the six sampling intervals. (b-c) Interpolated concentrations of nitrate and dissolved iron[Resing et al., 2015]. (d-e) Community composition based on HPLC pigment analysis. 

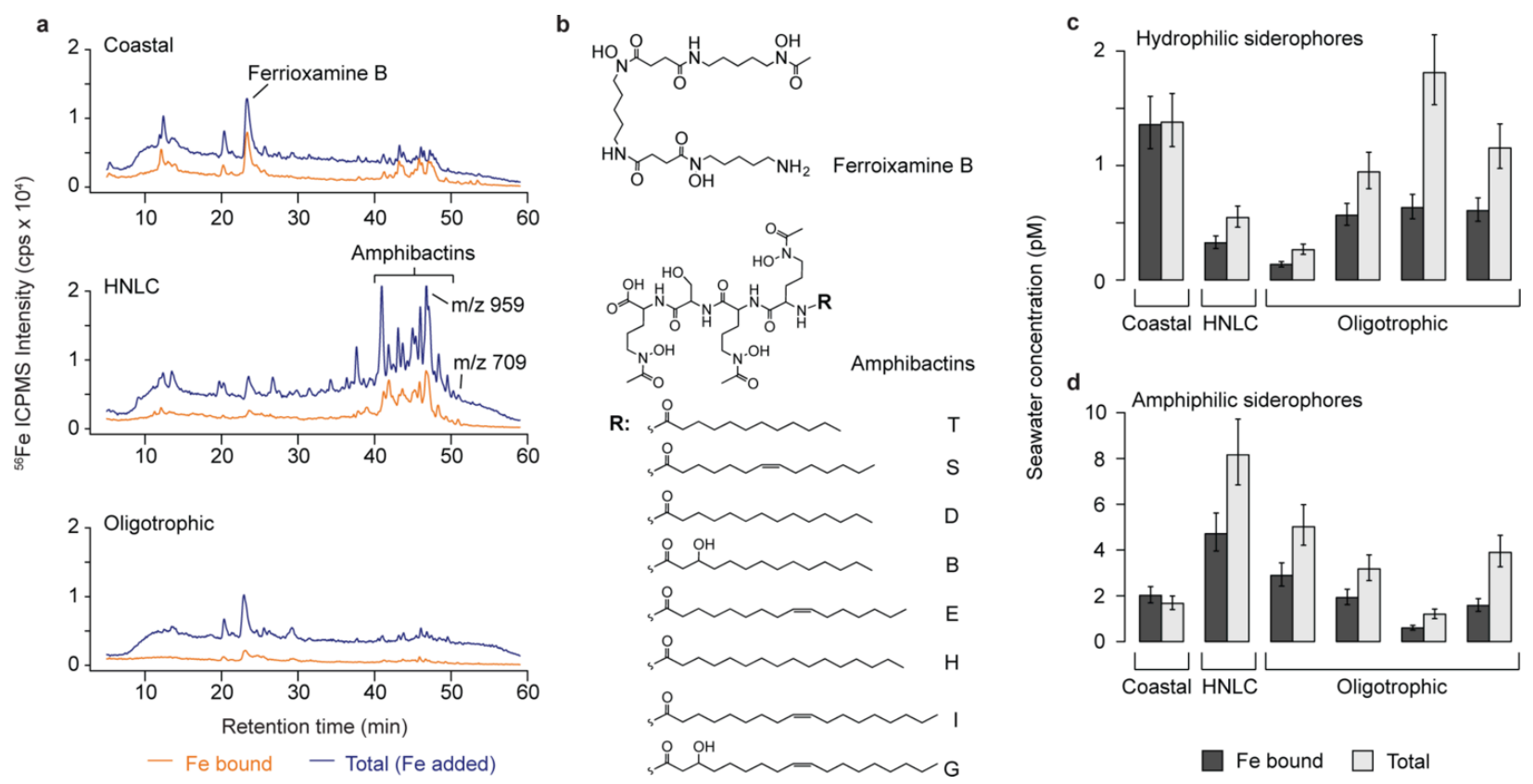

Figure 4.2: (a) Representative LC-ICPMS chromatograms of samples collected from the coastal, HNLC, and oligotrophic surface ocean. The orange line indicates ligands bound to naturally occurring iron, while the blue line indicates total ligand after addition of excess iron to saturate uncomplexed ligands. The major peaks at 23 minutes and between 40-50 minutes were identified as the siderophores ferrioxamine B and amphibactins B-T (structures shown in panel b). (c,d) Concentrations of hydrophilic siderophores (eluting between 18-28 minutes) and amphiphilic siderophores (eluting between 35-55 minutes). These total concentrations are anti-correlated $\left(\mathrm{r}^{2}=\right.$ $0.65)$. Error bars represent the analytical uncertainty $( \pm 2 \sigma)$. 


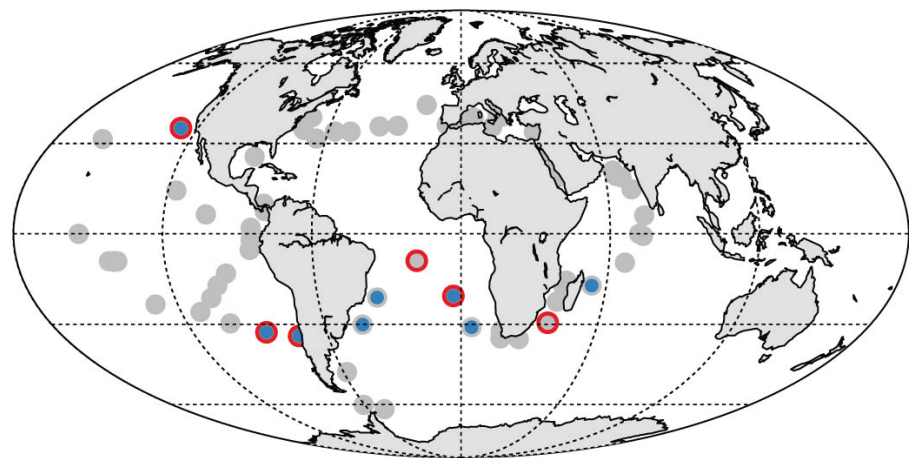

- ABO_2093 NRPS homologue present

- ABO_2092 NRPS homologue present

- Genes below detection limit

Figure 4.3: Geographic locations of nonribosomal peptide synthetase (NRPS) amphibactin biosynthesis genes within the Tara Ocean Microbial Reference Catalog. 


\subsection{Supplementary Figures and Tables:}

Table S4.1: Full list of iron compounds detected by LC-ICPMS and LC-ESIMS

\begin{tabular}{|c|c|c|c|c|c|c|c|}
\hline Peak & $\begin{array}{c}\text { Retention } \\
\text { Time (min) }\end{array}$ & $\begin{array}{c}54 \mathrm{Fe} \\
\text { complex } \\
\mathrm{m} / \mathrm{z}\end{array}$ & $\begin{array}{c}56 \mathrm{Fe} \\
\text { complex } \\
\mathrm{m} / \mathrm{z} \\
\end{array}$ & $\begin{array}{c}\text { Iron free } \\
\text { compound } \\
\mathrm{m} / \mathrm{z}\end{array}$ & ID & $\mathrm{MS}^{2} ?$ & $\begin{array}{c}57 \mathrm{Fe} \\
\text { validated? }\end{array}$ \\
\hline $\mathrm{a}$ & 11.9 & & & & & & \\
\hline b & 12.4 & & & & & & \\
\hline $\mathrm{c}$ & 13.5 & & & & & & \\
\hline d & 18.4 & & & & & & \\
\hline $\mathrm{e}$ & 19.7 & & & & & & \\
\hline $\mathrm{f}$ & 20.2 & & & & & & \\
\hline $\mathrm{g}$ & 22.9 & 612.278 & 614.273 & 561.361 & $\begin{array}{c}\text { ferrioxamine } \\
\text { B }\end{array}$ & Yes & No \\
\hline $\mathrm{h}$ & 25.6 & & & & & & \\
\hline $\mathrm{i}$ & 26.7 & & & & & & \\
\hline $\mathrm{j}$ & 29.2 & & & & & & \\
\hline $\mathrm{k}$ & 31.4 & & & & & & \\
\hline 1 & 34.3 & & & & & & \\
\hline $\mathrm{m}$ & 36.4 & & & & & & \\
\hline $\mathrm{n}$ & 37.6 & & & & & & \\
\hline o & 40.9 & 855.388 & 857.383 & 804.472 & $\begin{array}{c}\text { Amphibactin } \\
\mathrm{T}\end{array}$ & Yes & No \\
\hline $\mathrm{p}$ & 41.8 & & & & & & \\
\hline q & 43.1 & 899.415 & 901.410 & 848.499 & $\begin{array}{c}\text { Amphibactin } \\
\text { B } \\
\text { Amphibactin }\end{array}$ & No & No \\
\hline $\mathrm{r}$ & 43.7 & 881.404 & 883.399 & 830.488 & $\mathrm{~S}$ & No & Yes \\
\hline $\mathrm{s}$ & 45.0 & & & & & & \\
\hline $\mathrm{t}$ & 45.4 & & & & & & \\
\hline $\mathrm{u}$ & 46.0 & 883.419 & 885.414 & 832.503 & $\begin{array}{c}\text { Amphibactin } \\
\text { D }\end{array}$ & Yes & Yes \\
\hline $\mathrm{v}$ & 46.8 & 957.435 & 959.43 & 906.519 & $\begin{array}{c}\text { Unknown } \\
\text { Amphibactin }\end{array}$ & Yes & Yes \\
\hline w & 47.1 & 909.434 & 911.429 & 858.518 & $\begin{array}{c}\mathrm{E} \\
\text { Amphibactin }\end{array}$ & Yes & Yes \\
\hline $\mathrm{x}$ & 48.4 & 953.461 & 955.456 & 902.545 & $\begin{array}{c}\mathrm{G} \\
\text { Amphibactin }\end{array}$ & No & No \\
\hline $\mathrm{y}$ & 49.5 & 911.450 & 913.445 & 860.534 & $\begin{array}{c}\mathrm{H} \\
\text { Amphibactin }\end{array}$ & Yes & Yes \\
\hline $\mathrm{z}$ & 50.3 & 937.467 & 939.462 & 886.551 & I & Yes & Yes \\
\hline aa & 50.9 & 707.377 & 709.372 & 656.461 & Unknown & Yes & Yes \\
\hline
\end{tabular}


Table S4.2: Location of South Pacific Samples collected during US GEOTRACES EPZT Cruise

\begin{tabular}{lllllll}
\hline Sample & \multirow{2}{*}{ Conditions } & \multicolumn{2}{c}{ Transect Start } & \multicolumn{2}{c}{ Transect End } & Depth \\
& & Latitude & Longitude & Latitude & Longitude & \\
\hline Sample 1 & Coastal upwelling & $12.047^{\circ} \mathrm{S}$ & $77.432^{\circ} \mathrm{W}$ & $12.001^{\circ} \mathrm{S}$ & $86.524^{\circ} \mathrm{W}$ & $3 \mathrm{~m}$ \\
Sample 2 & HNLC & $12.001^{\circ} \mathrm{S}$ & $86.524^{\circ} \mathrm{W}$ & $13.96^{\circ} \mathrm{S}$ & $99.049^{\circ} \mathrm{W}$ & $3 \mathrm{~m}$ \\
Sample 3 & Oligotrophic & $13.96^{\circ} \mathrm{S}$ & $99.049^{\circ} \mathrm{W}$ & $14.383^{\circ} \mathrm{S}$ & $117.534^{\circ} \mathrm{W}$ & $3 \mathrm{~m}$ \\
Sample 4 & Oligotrophic & $14.383^{\circ} \mathrm{S}$ & $117.534^{\circ} \mathrm{W}$ & $11.64^{\circ} \mathrm{S}$ & $132.563^{\circ} \mathrm{W}$ & $3 \mathrm{~m}$ \\
Sample 5 & Oligotrophic & $11.64^{\circ} \mathrm{S}$ & $132.563^{\circ} \mathrm{W}$ & $11.073^{\circ} \mathrm{S}$ & $143.003^{\circ} \mathrm{W}$ & $3 \mathrm{~m}$ \\
Sample 6 & Oligotrophic & $11.073^{\circ} \mathrm{S}$ & $143.003^{\circ} \mathrm{W}$ & $10.518^{\circ} \mathrm{S}$ & $152.079^{\circ} \mathrm{W}$ & $3 \mathrm{~m}$
\end{tabular}



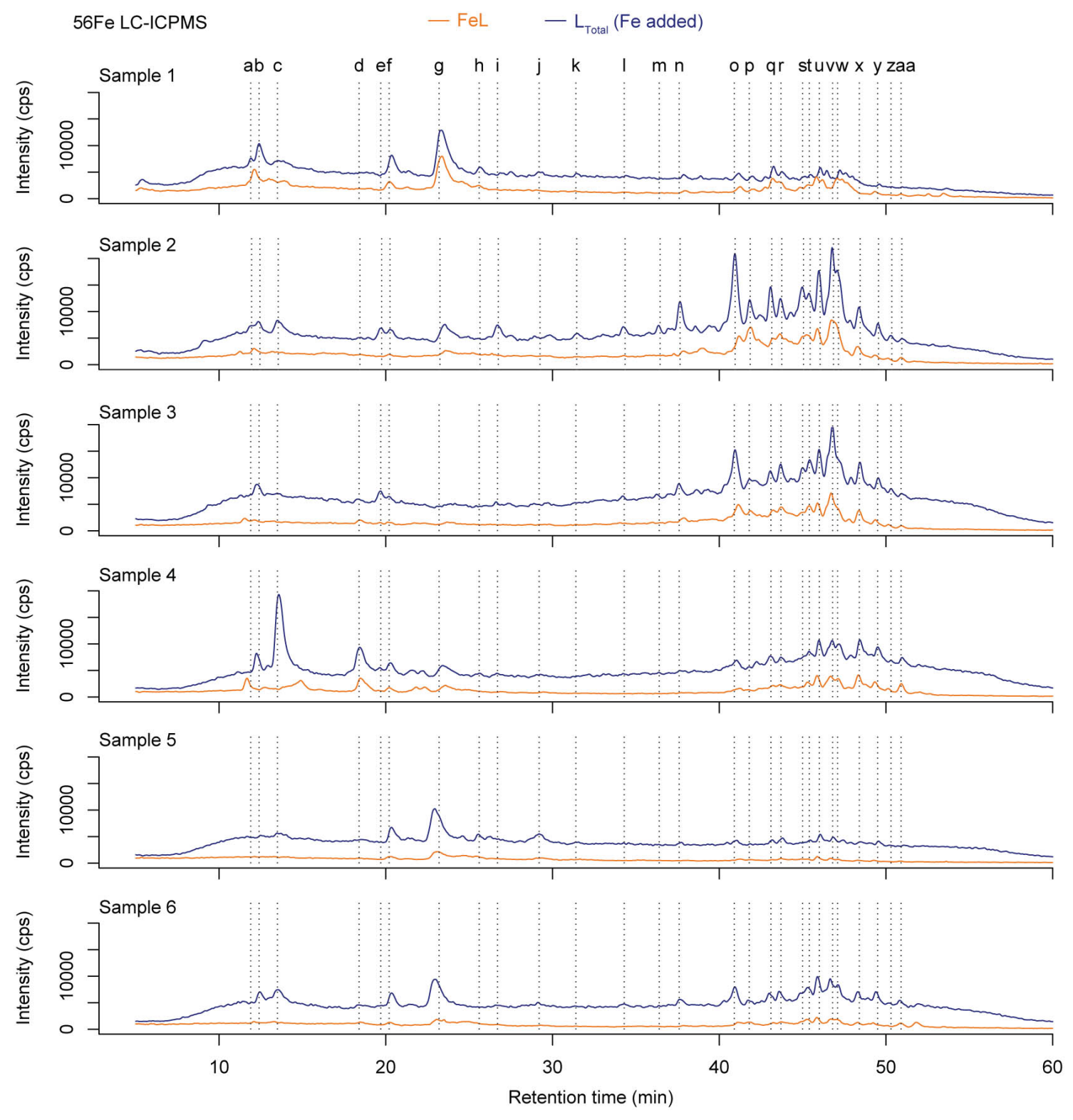

Figure S4.1: ${ }^{56} \mathrm{Fe}$ LC-ICPMS chromatograms from the six samples collected from the coastal environment (sample 1), the HNLC region (sample 2), and the oligotrophic region (samples 3-6). All signals have been standardized to account for differences in preconcentration. Ligands that were bound to naturally occurring iron were initially detected (orange line). After addition of excess iron to each sample, the uncomplexed ligands were saturated with iron and the total ligand pool was detected (blue line). 
a

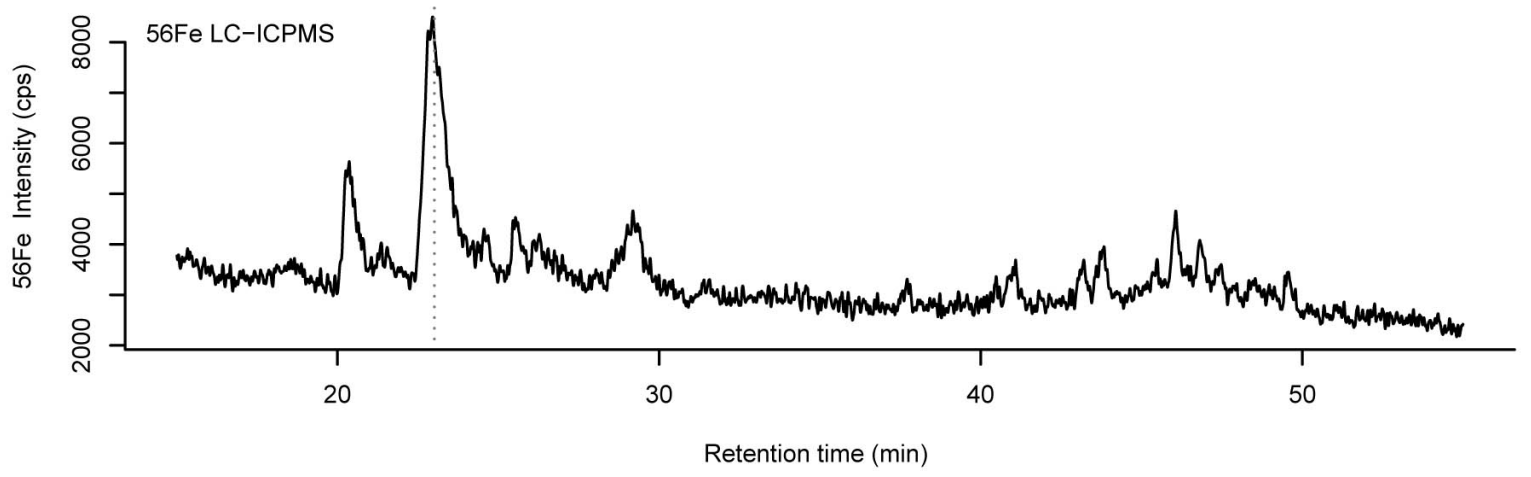

b

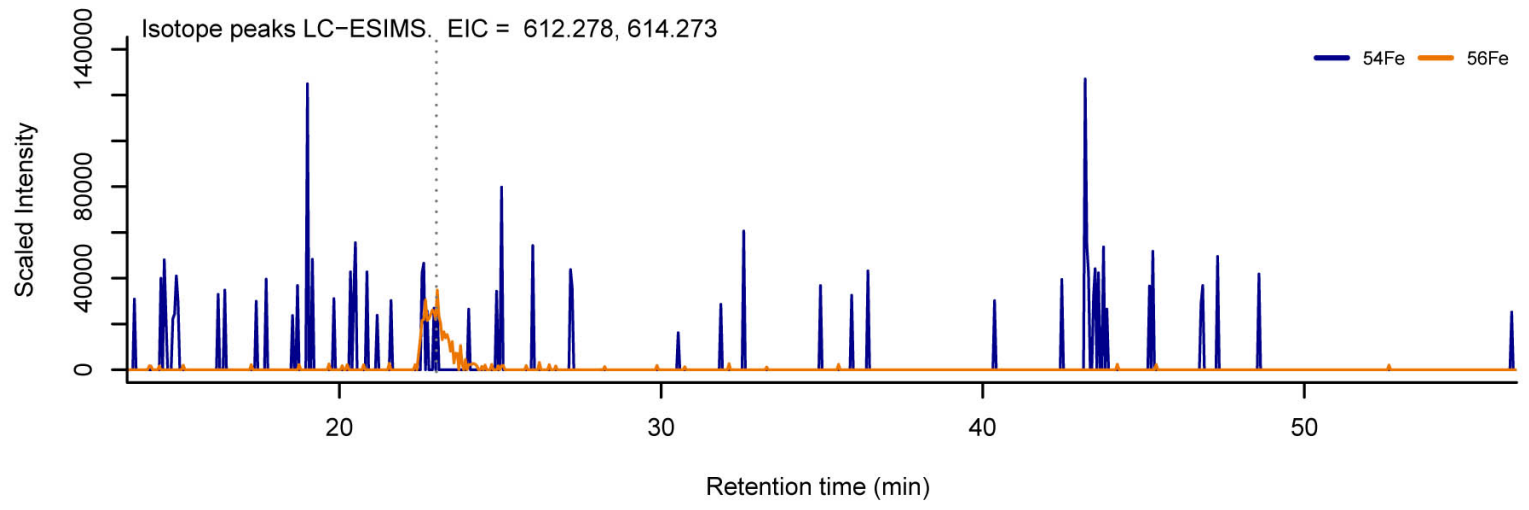

C

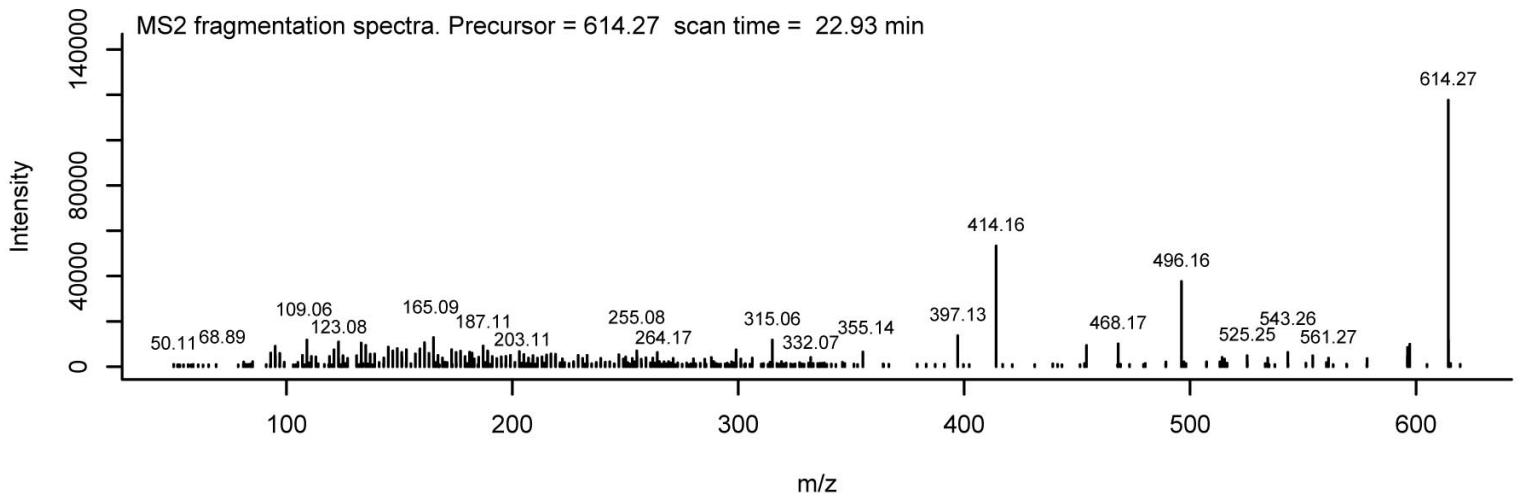

Figure S4.2: Ferrioxamine B characterization. (a) ${ }^{56}$ Fe LC-ICPMS chromatogram. (b) Extracted ion chromatograms (EIC) from positive mode LC-ESIMS chromatograms. Blue lines correspond to the ${ }^{54} \mathrm{Fe}$-siderophore $[\mathrm{M}+\mathrm{H}]^{+}$isotopologue and orange lines correspond to the ${ }^{56} \mathrm{Fe}-$ siderophore $[\mathrm{M}+\mathrm{H}]^{+}$isotopologue. The intensity of the heavy iron isotopologue has been scaled by the natural abundance ratio of ${ }^{56} \mathrm{Fe} /{ }^{54} \mathrm{Fe}$ (divided by 15.7) so that the isotopologues overlap. (c) $\mathrm{MS}^{2}$ fragmentation spectra of the ${ }^{56} \mathrm{Fe}$ isotopologues. 
a

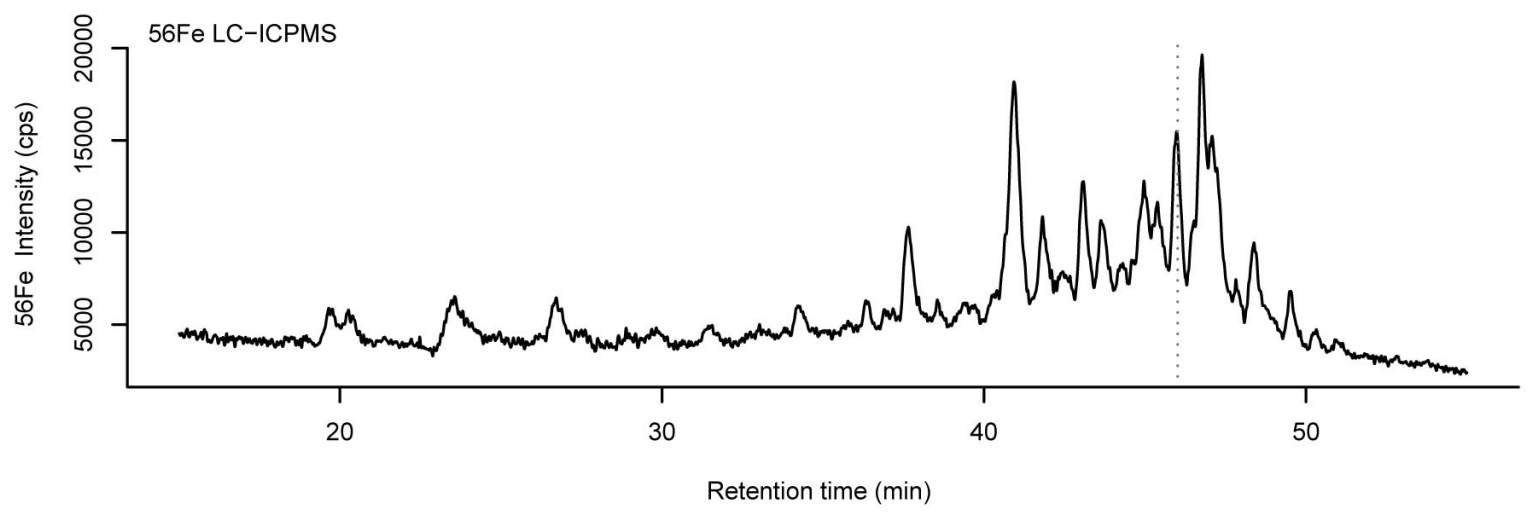

b

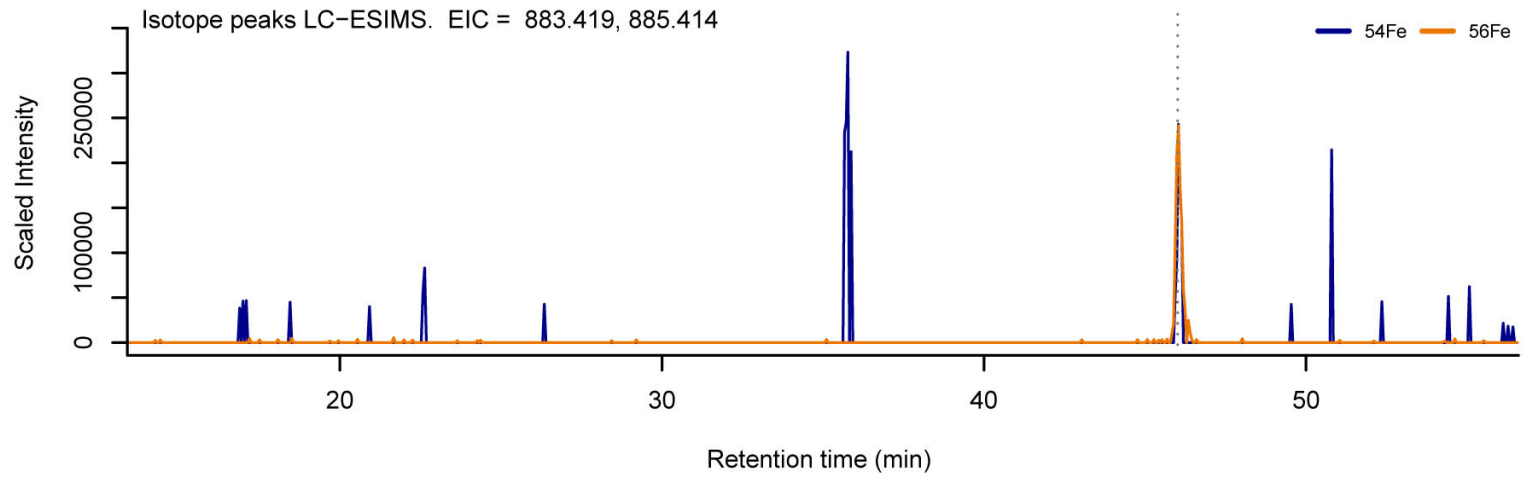

C

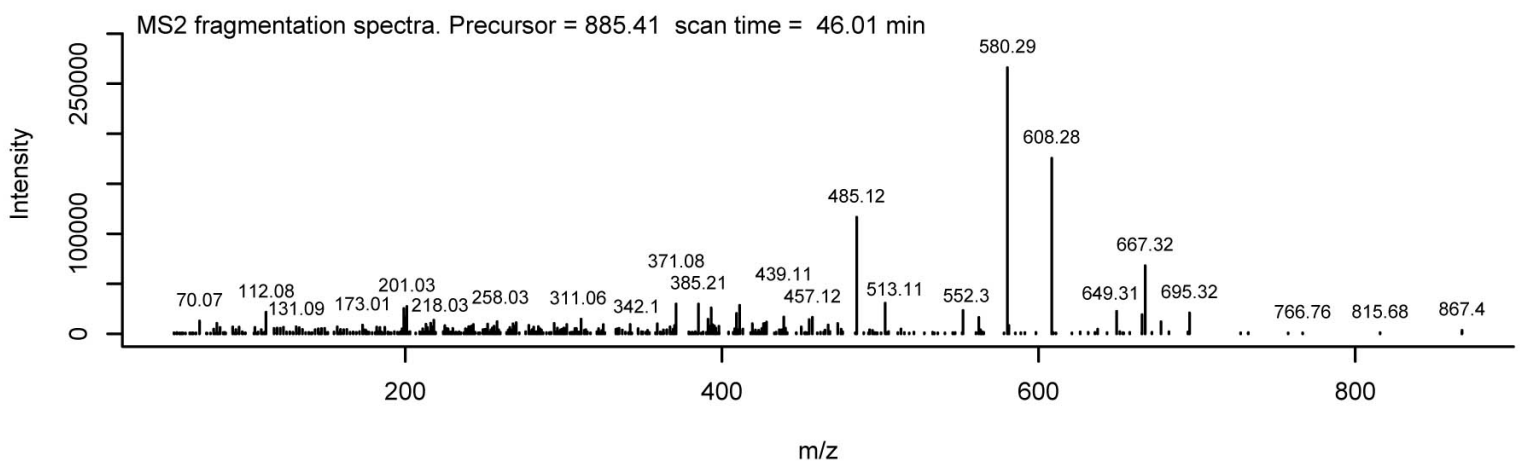

Figure S4.3: Ampibactin D characterization. The subpanels are the same as presented in Extended Data Figure 2. 
a

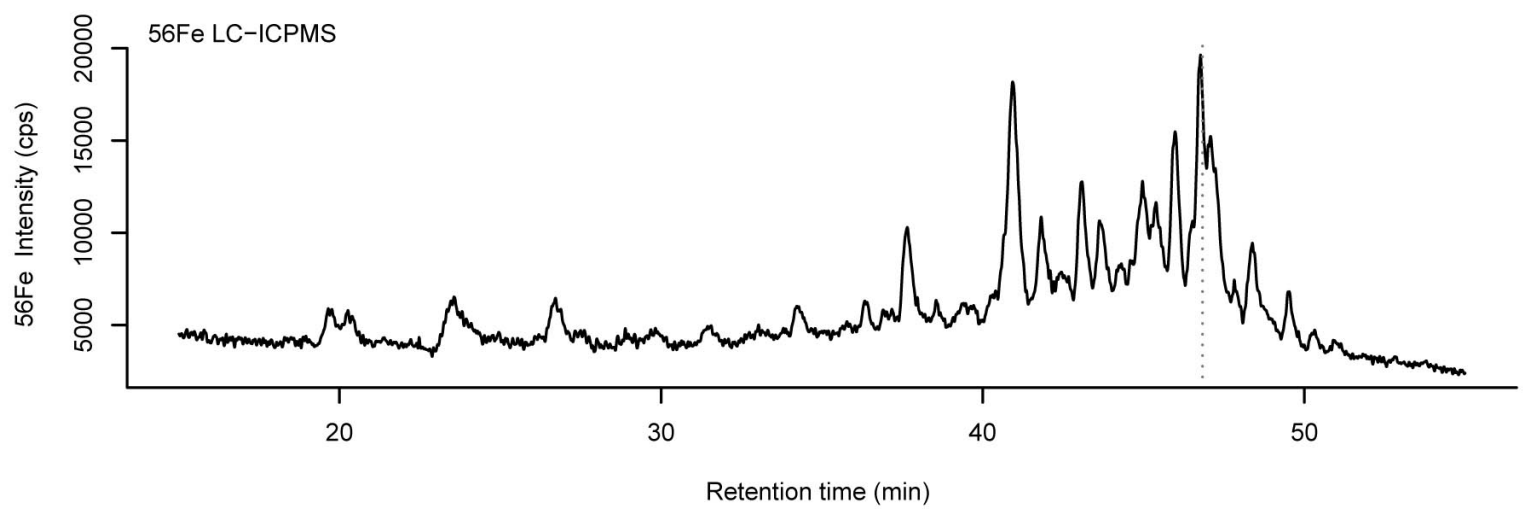

b

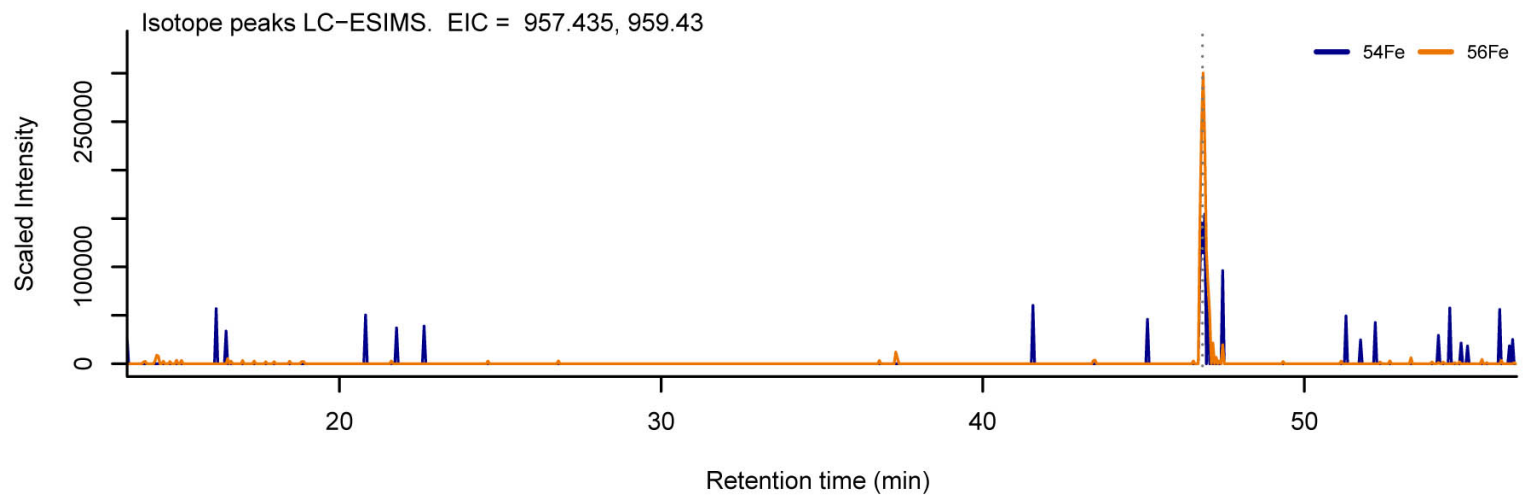

C

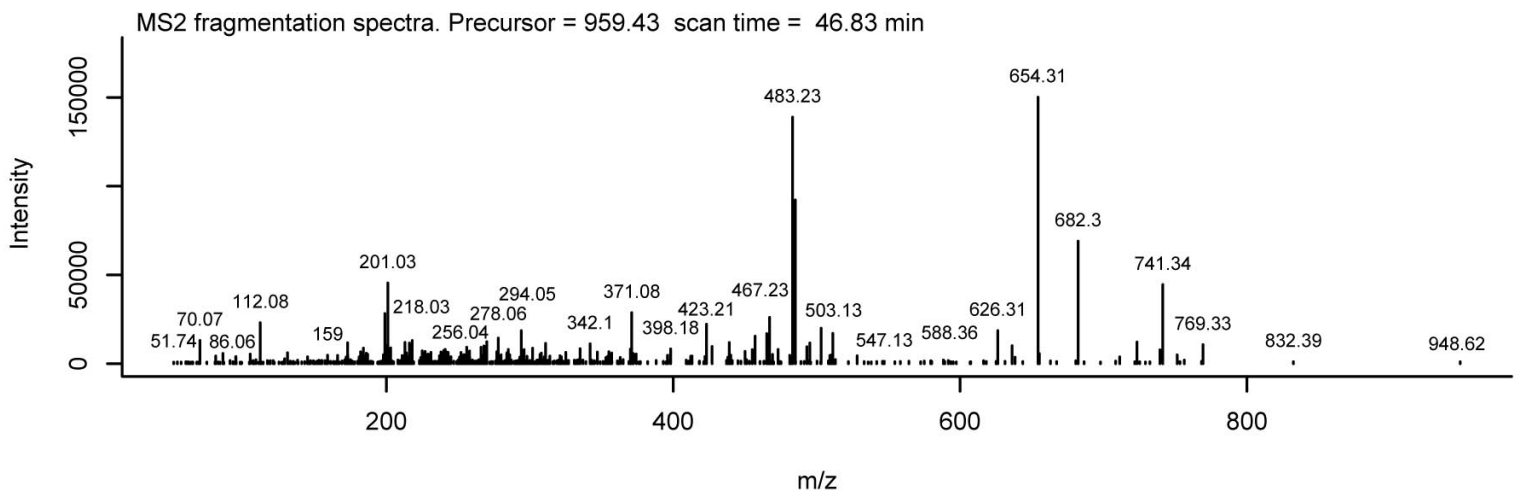

Figure S4.4: Unknown compound $(959 \mathrm{~m} / \mathrm{z})$ characterization. The subpanels are the same as presented in Extended Data Figure 2. 
a

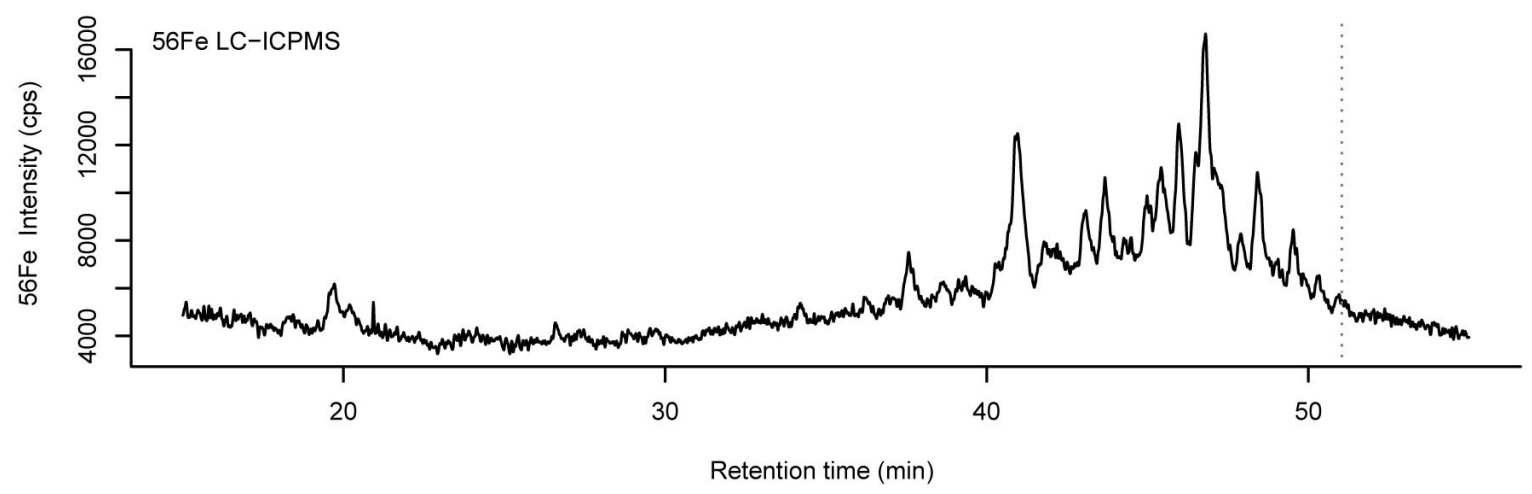

b

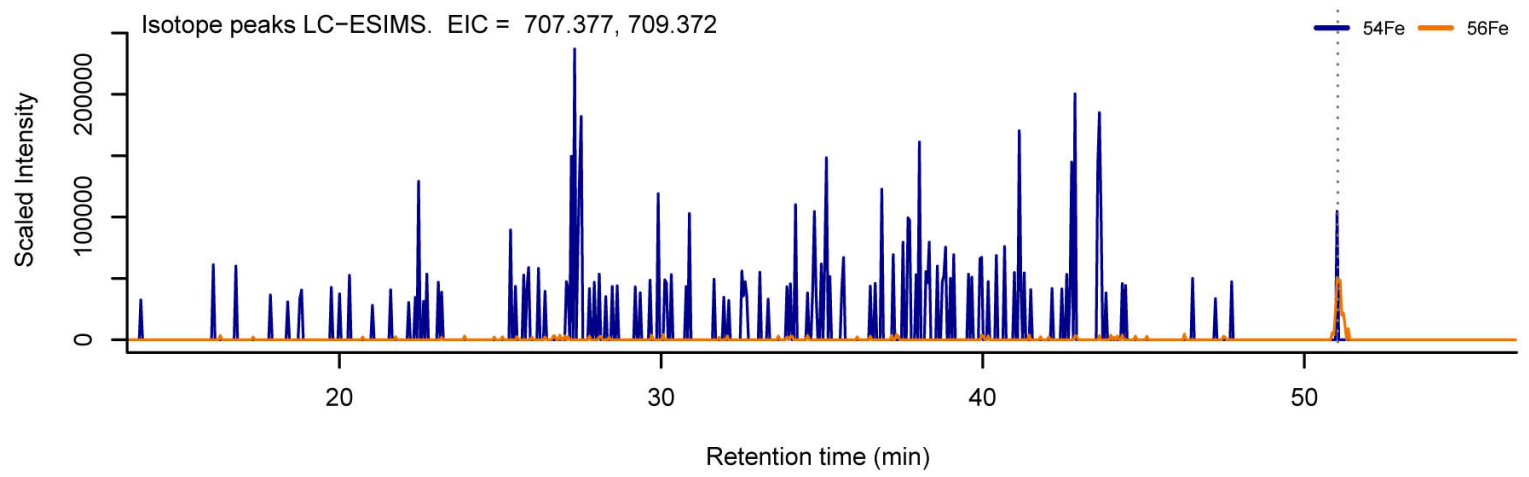

C

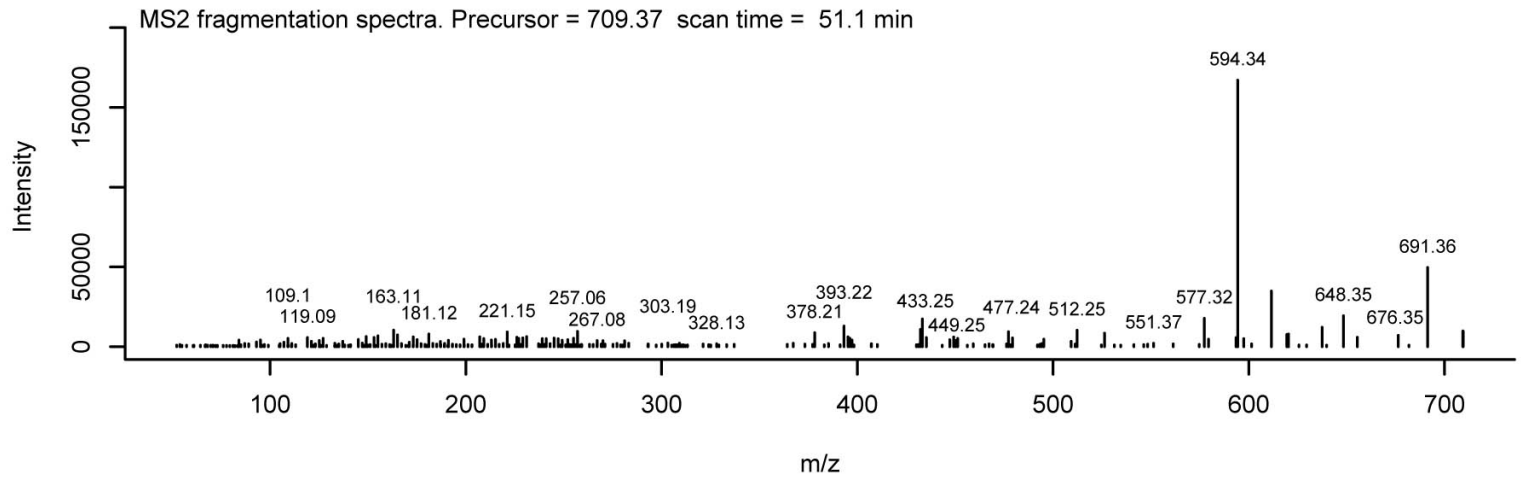

Figure S4.5: Unknown compound $(709 \mathrm{~m} / \mathrm{z})$ characterization. The subpanels are the same as presented in Extended Data Figure 2. 


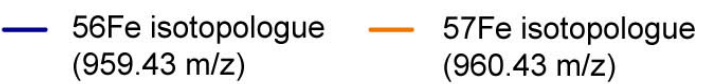

a

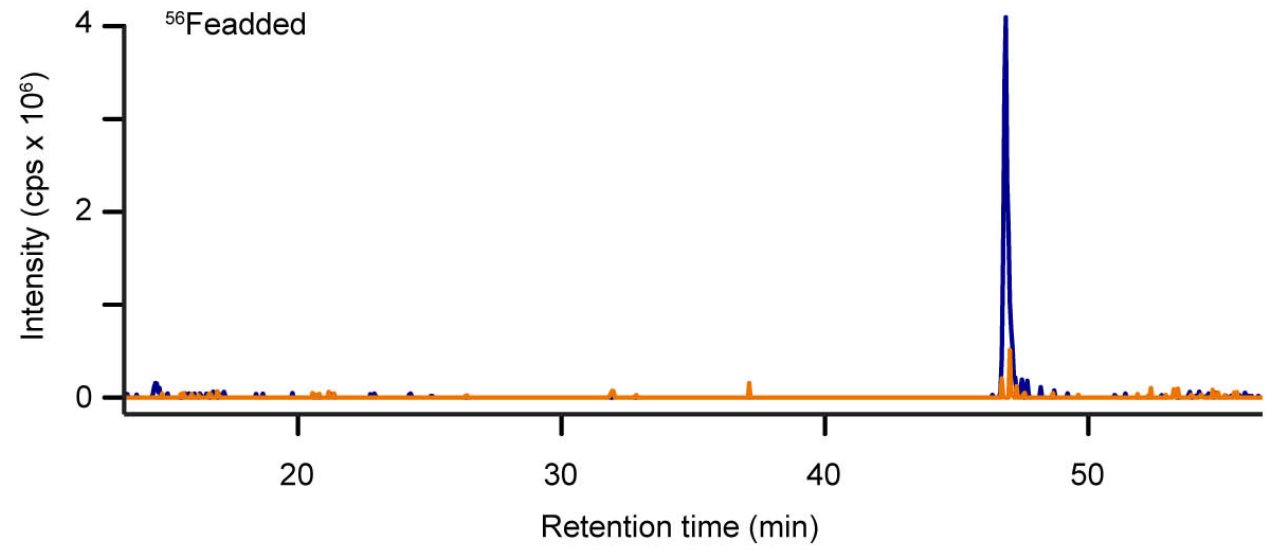

b

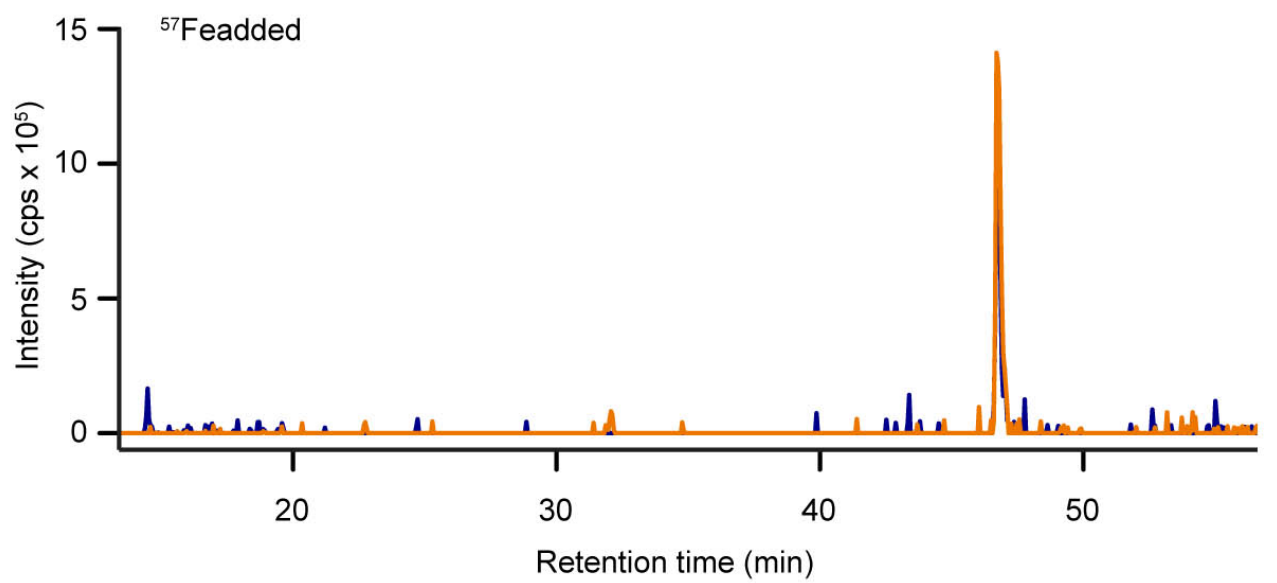

Figure S4.6: Example of ${ }^{57} \mathrm{Fe}$ validation of siderophore masses for unknown compound (959 $\mathrm{m} / \mathrm{z}$ ). Extracted ion chromatograms from LC-ESIMS chromatograms of (a) ${ }^{56} \mathrm{Fe}$ spiked samples and (b) ${ }^{57} \mathrm{Fe}$ spiked samples. Blue lines correspond to the ${ }^{56} \mathrm{Fe}$-siderophore $[\mathrm{M}+\mathrm{H}]^{+} \mathrm{m} / \mathrm{z}$ and orange lines correspond to the ${ }^{57} \mathrm{Fe}$-siderophore $[\mathrm{M}+\mathrm{H}]^{+} \mathrm{m} / \mathrm{z}$. Features that contain iron show a difference in the relative abundance of the two isotopologues between the treatments. 
a ABO_2093 Amphibactin NRPS homologs

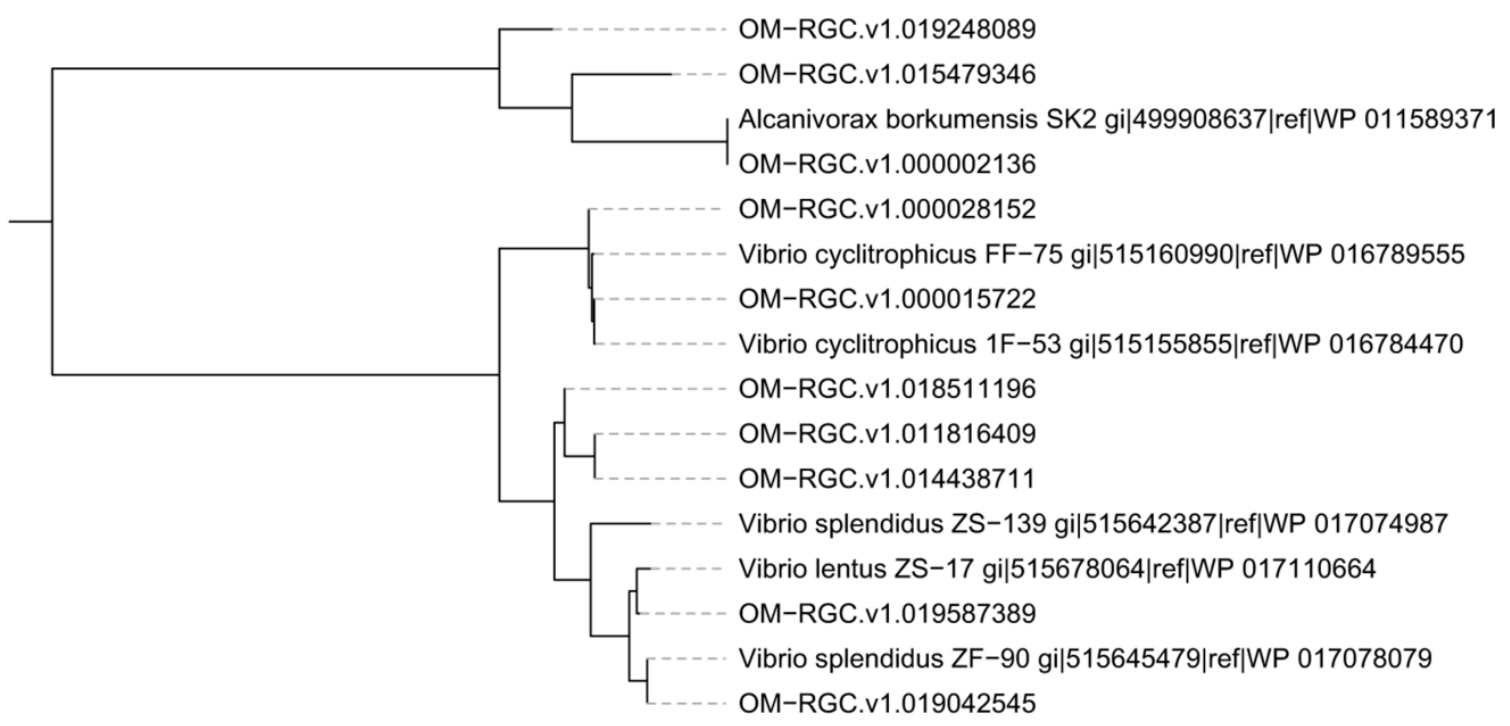

b ABO_2092 Amphibactin NRPS homologs

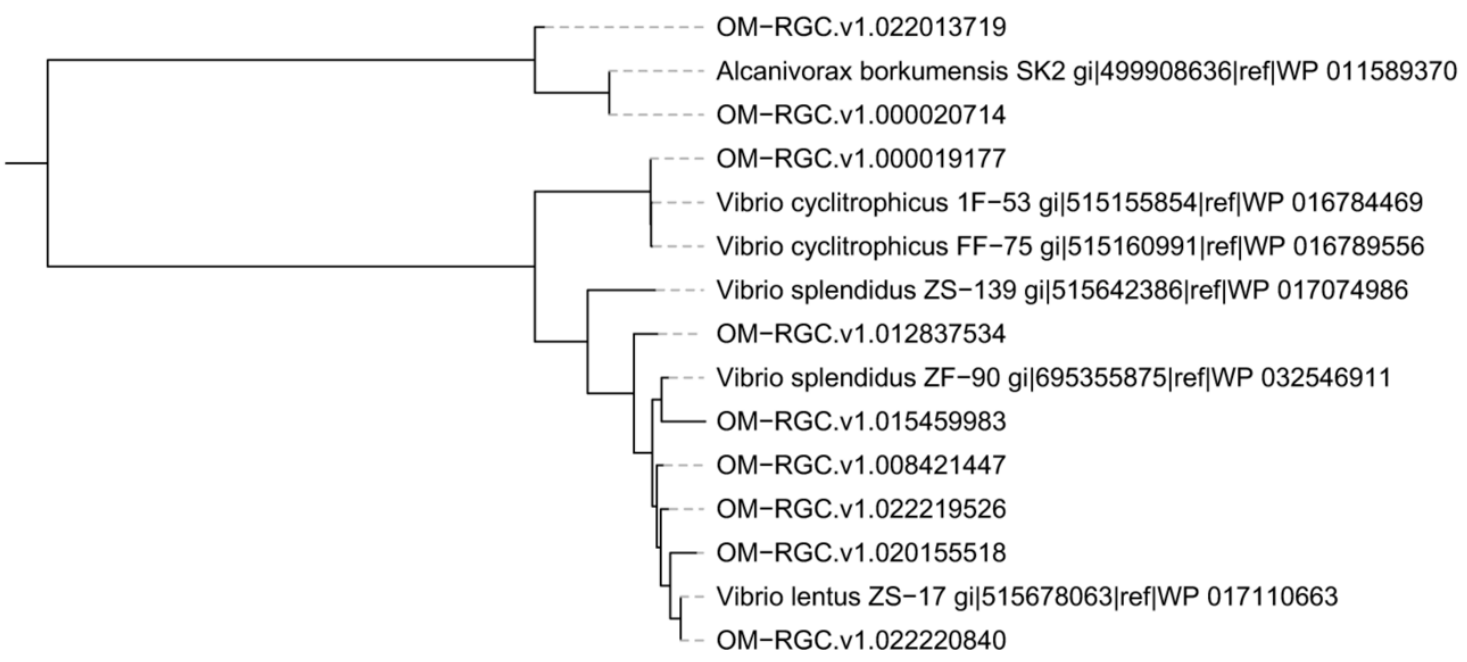

Figure S4.7: Phylogenetic tree of amphibactin synthesis genes identified from cultured Gammaproteobacteria and homologs identified in the Tara OMRGC. 

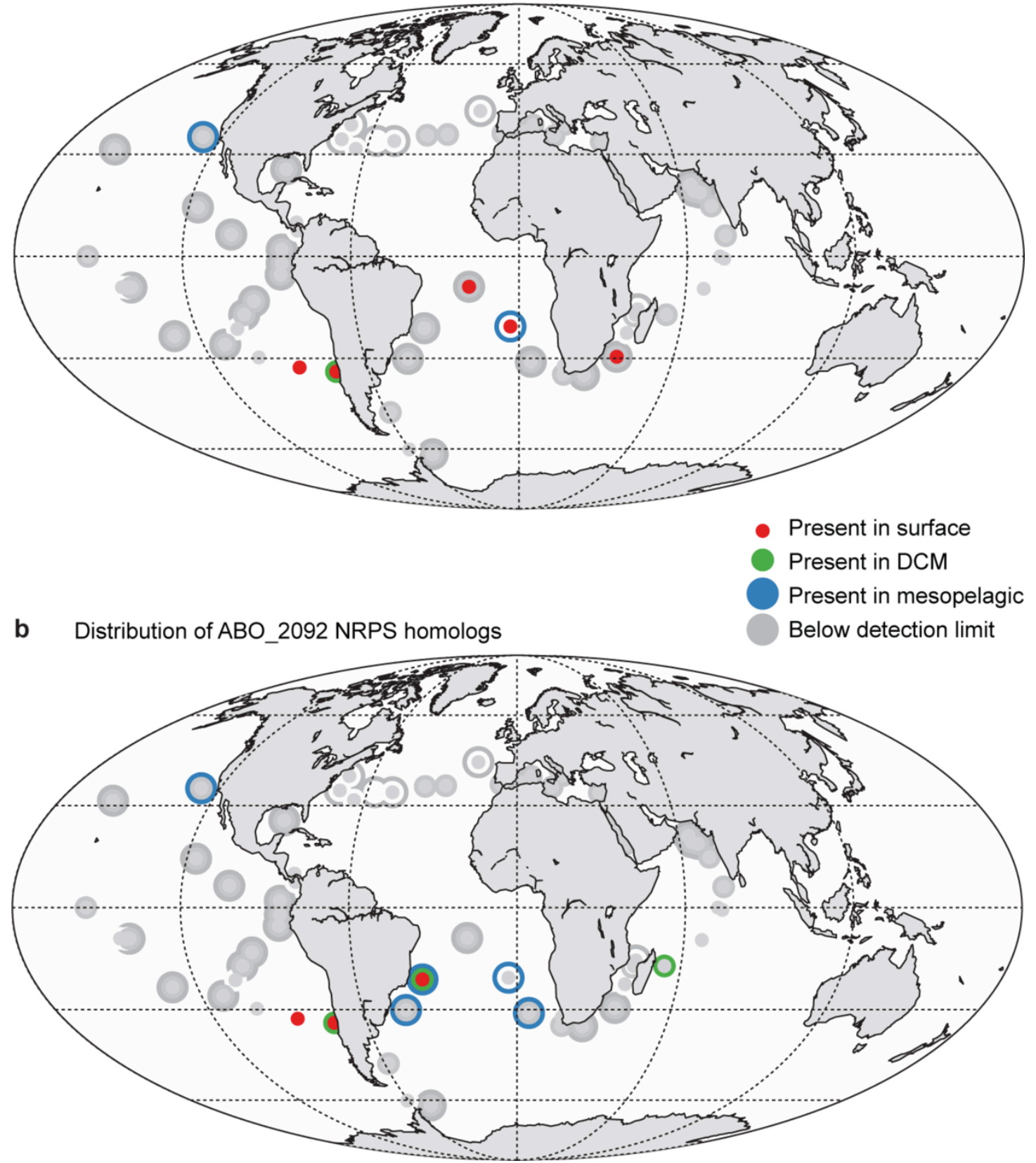

Figure S4.8: Depth distributions of amphibactin nonribosomal peptide synthetase (NRPS) genes within the Tara OMRGC in the surface (5m depth), deep chlorophyll maximum (DCM, 30$112 \mathrm{~m}$ depth) and mesopelagic ( $380-820 \mathrm{~m}$ depth) layers. 


\section{Appendix 4.1: Ni and Cu compounds across the Eastern Tropical South Pacific}

One of the prime advantages to using a quadrupole ICPMS for speciation analyses is that the fast scanning speed allows for many elemental isotopes to be monitored simultaneously (barring those that suffer from interferences). While this chapter focuses on iron, additional data was collected for other transition metals, including $\mathrm{Ni}$ and $\mathrm{Cu}$. Several $\mathrm{Ni}$ and $\mathrm{Cu}$ compounds were detected and characterized from the six samples collected on the GEOTRACES cruise. The LCICPMS chromatograms for these metals revealed the presence of a compound eluting at $40 \mathrm{~min}$ that binds both $\mathrm{Ni}$ and $\mathrm{Cu}$ and elutes at $40 \mathrm{~min}$. Using isotope assisted screening by LC-ESIMS, the parent ion was determined (Table A4-1). The metal free apo form of the complex $[\mathrm{M}+\mathrm{H}]^{+}$ with $\mathrm{m} / \mathrm{z} 511$ was not detected. The metal bound forms of these compounds were most abundant in the last two samples (5 and 6), collected from the oligotrophic region near Tahiti.

In addition, three compounds were detected in samples 4 and 6 that bind $\mathrm{Ni}$ exclusively and their masses were determined by LC-ESIMS. Since the stability of $\mathrm{Cu}$ complexes is typically higher than for Ni complexes, the three Ni compounds (Fig A4-1 peaks b-d) are likely kinetically inert to metal exchange. The apo-form was not observed for any of these compounds. The two peaks with $\left[\mathrm{M}+{ }^{60} \mathrm{Ni}-\mathrm{H}\right]^{+}$of 700.275 and $714.291 \mathrm{~m} / \mathrm{z}$ are structurally related based on their $\mathrm{MS}^{2}$ fragmentation patterns, differing by $\mathrm{CH}_{2}$. At this stage, these $\mathrm{Ni}$ and $\mathrm{Cu}$ compounds have not been linked to known compounds produced by organisms. The discovery of these compoudns in the oligotrophic, low metal ocean may hint that they are cofactors used in metabolisms that substitute $\mathrm{Ni}$ or $\mathrm{Cu}$ for other elements that are scarce in this environment such as $\mathrm{Fe}$. 

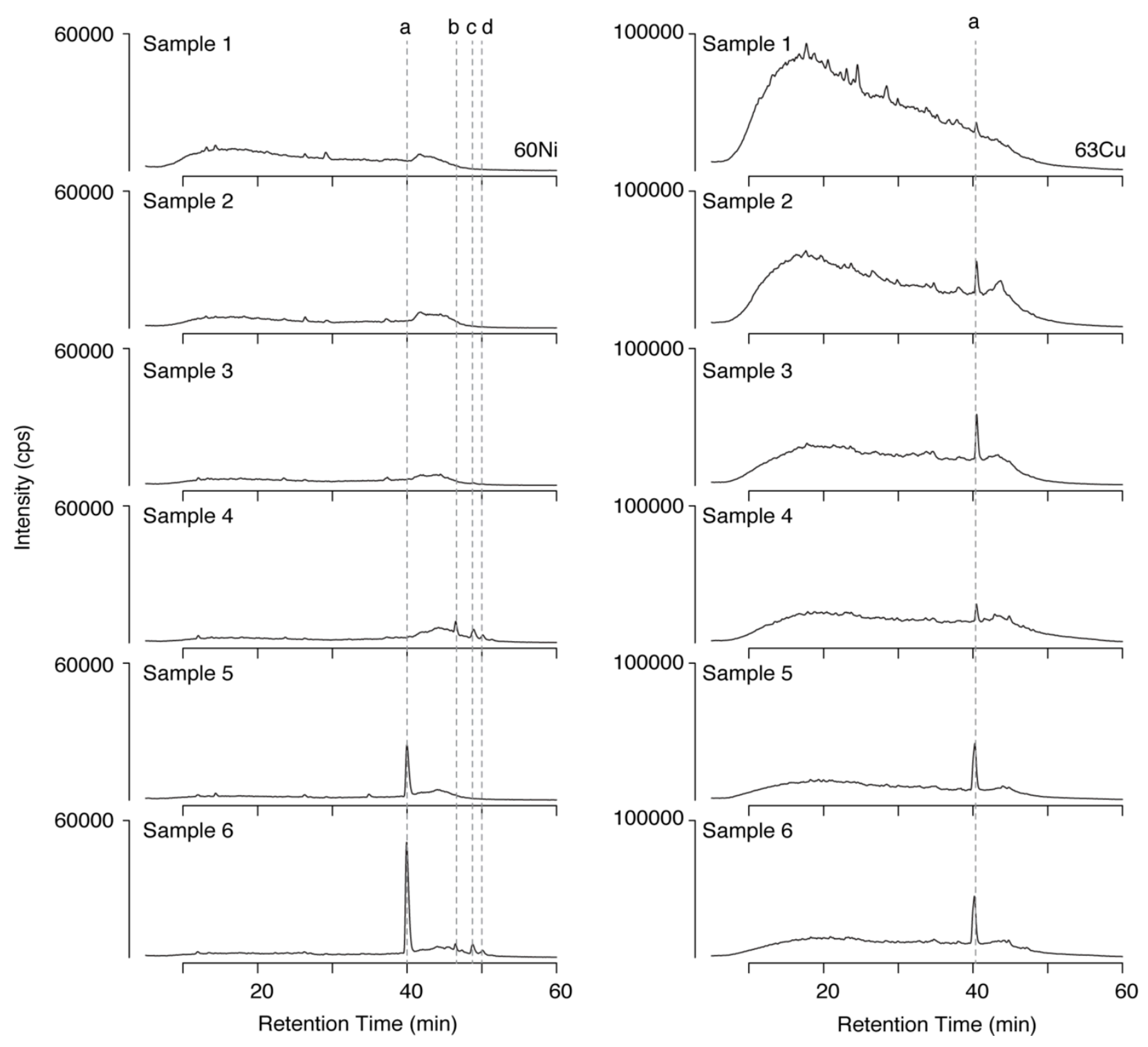

Figure A4.1: ${ }^{60} \mathrm{Ni}$ (left) and ${ }^{63} \mathrm{Cu}$ (right) LC-ICPMS chromatograms from the six samples collected from the coastal environment (sample 1), the HNLC region (sample 2), and the oligotrophic region (samples 3-6). All signals have been standardized to account for differences in preconcentration. Dashed lines indicate retention times of identified compounds. 
Table A4.1: Ni and Cu compounds identified across the GEOTRACES EPZT transect

\begin{tabular}{cccccccc}
\hline Peak & $\begin{array}{c}\text { Retention Time } \\
(\mathrm{min})\end{array}$ & $\begin{array}{c}{[\mathrm{M}+\mathrm{H}]^{+}} \\
\mathrm{m} / \mathrm{z}\end{array}$ & $\begin{array}{c}{[\mathrm{M}+58 \mathrm{Ni}-\mathrm{H}]^{+}} \\
\mathrm{m} / \mathrm{z}\end{array}$ & $\begin{array}{c}{[\mathrm{M}+60 \mathrm{Ni}-\mathrm{H}]^{+}} \\
\mathrm{m} / \mathrm{z}\end{array}$ & $\begin{array}{c}{[\mathrm{M}+63 \mathrm{Cu}-\mathrm{H}]^{+}} \\
\mathrm{m} / \mathrm{z}\end{array}$ & $\begin{array}{c}{[\mathrm{M}+65 \mathrm{Cu}-\mathrm{H}]^{+}} \\
\mathrm{m} / \mathrm{z}\end{array}$ \\
\hline a & 39.8 & 511.095 & 567.015 & 569.011 & 572.009 & 574.007 \\
b & 46.4 & 591.143 & 649.148 & 651.143 & $\mathrm{~N} / \mathrm{A}$ & $\mathrm{N} / \mathrm{A}$ \\
$\mathrm{c}$ & 48.6 & 640.275 & 698.28 & 700.275 & $\mathrm{~N} / \mathrm{A}$ & $\mathrm{N} / \mathrm{A}$ \\
d & 49.9 & 654.291 & 712.295 & 714.291 & N/A & N/A
\end{tabular}




\title{
Chapter 5: Diversity of metal ligands in the California Upwelling system
}

\begin{abstract}
:
The California current is a dynamic ocean environment characterized by strong seasonal upwelling that fuels intense phytoplankton productivity during summer months. Iron supply and recycling is known to play an important role in the timing and intensity of biological productivity in this region. Several modes of iron cycling have been observed, including enhanced supply of iron to the surface through upwelling over wide continental shelf regions, rapid removal in surface waters, and the recycling of iron by microbes and zooplankton in the euphotic zone, which sustains nitrate and phosphate utilization. Yet little is known about the fundamental processes that drive iron cycling or the types of organic ligands that mediate them. This chapter provides the first identification of iron binding ligands that play a role in these processes within the California current.
\end{abstract}

Siderophores were detected in surface waters, including ferrioxamine B, amphibactins, and an unknown compound with $\mathrm{m} / \mathrm{z}$ of 709 at concentrations ranging from 1-7pM. Synechobactins were also detected within nepheloid layers near the benthic boundary along the continental shelf, suggesting a potential role of siderophores for iron acquisition and iron remobilization within coastal ocean sediments. In the mesopelagic, the major iron binding ligands detected were chromatographically unresolved polar compounds, likely sourced from the degradation of organic matter in the water column or sediments and reaching concentrations of up to $1 \mathrm{nM}$ near the coastal benthic boundary. Grazing experiments revealed that copepods are a source of the siderophores ferrioxamine $\mathrm{E}$ and $\mathrm{G}$, likely produced by associated heterotrophic bacteria, as well as numerous structurally related unknown organic ligands of $\mathrm{m} / \mathrm{z} 876,902,854,884$, and 882. Finally, zooplankton grazing of phytoplankton released large amounts of another unknown compound with $\mathrm{m} / \mathrm{z}$ of 968 . These findings indicate that much of the iron regenerated by zooplankton grazing is associated with specific biomolecules, including siderophores, which likely have important consequences for the bioavailability of regenerated iron. Together, these results reveal that iron speciation is highly variable due to the release of structurally distinct iron binding compounds by microbes that are adapted to specific ecological niches, whether they are sedimentary, nutrient rich, iron deficient, or associated with eukaryotic hosts (e.g. zooplankton). 
These compounds have a direct impact on iron bioavailability and recycling, particularly in the case of siderophore production.

\subsection{Introduction:}

The California current system (CCS) is a highly productive eastern boundary of the Pacific Ocean with intense seasonal biological cycles that are driven by ocean chemistry and physics [Rykaczewski and Checkley, 2008; Checkley and Barth, 2009]. Beginning in the early spring, strong equator-ward winds drive Ekman transport of surface waters offshore and replacement with cold, salty deep water that is rich in nitrate, phosphate, and silicate, as well as trace metals [Biller and Bruland, 2013]. These nutrients fuel intense phytoplankton blooms that are sustained throughout the spring and summer. Metal availability, particularly iron, is a major factor that determines the extent of bloom formation and ecosystem community structure in these regions [Hutchins and Bruland, 1998; Bruland et al., 2001]. Over time, dissolved iron concentrations decrease faster than nitrate, potentially due to scavenging or biological uptake [Firme et al., 2003; Biller and Bruland, 2013; Biller et al., 2013]. Iron addition to the surface waters have been shown to enhance overall productivity in areas with low nitrate:Fe concentration ratios [Kirchman et al., 2000; King and Barbeau, 2007]. While these studies suggest that the amount of iron delivered to the surface ocean exerts a significant control over nutrient cycling and biological productivity, many questions remain about the processes that supply this iron and the rate at which it is removed.

The predominant source of iron to the water column in the CCS is from continental shelf sediments. Rivers deliver iron rich mud to these regions during winter flood events [Wheatcroft et al., 1997; Xu et al., 2002]. Upwelling over wide continental shelves causes particle resuspension and enriches these waters with dissolved iron, causing more intense phytoplankton blooms and greater nitrate drawdown in wide shelf coastal regions than in narrow shelf regions that are supplied with less iron [Chase et al., 2005; Lohan and Bruland, 2008; Biller and Bruland, 2013; Biller et al., 2013]. This nutrient rich upwelled water advects offshore, resulting in a transition zone between the coast and the offshore oligotrophic California current. This mixing region can be several hundred $\mathrm{km}$ wide, varying seasonally, and is dominated by 
mesoscale eddies that promote the offshore transport of nutrient rich coastally upwelled waters [Keith Johnson et al., 2005; Peterson et al., 2005, 2011]. Iron concentrations are typically low throughout this transition region $(<<1 \mathrm{nM})$ while high nitrate concentrations persist in some locations [Biller and Bruland, 2014]. Finally, further offshore lies the warm, less saline water of the California current, where iron and macronutrients have both been depleted and picocyanobacteria dominate primary productivity.

While the broad scale pattern of iron upwelling along the coast and iron limitation offshore have been well documented [Kirchman et al., 2000; Bruland et al., 2001; Firme et al., 2003; King and Barbeau, 2007; Biller et al., 2013], the processes that add and recycle metals in these regions remain poorly constrained. Nearly all of the dissolved iron in the CCS is complexed by organic compounds of unknown source and composition [Buck et al., 2007; Bundy et al., 2014, 2015a]. These compounds drive many aspects of iron cycling, including (1) the dissolution of iron from detrital particles and sediments (2) the scavenging of iron from the water column by sinking particles and organic flocs (3) the iron uptake rates of marine microbes and (4) photochemical reduction and oxidation rates of iron. Uncovering the fundamental chemical and biological processes that transfer metals between sediments, biota and dissolved phases is necessary to predict changes in metal cycling that impact community composition, primary productivity, and carbon fixation rates in these regions.

The composition of ligands is highly variable in the coastal environment, with concentrations and binding strengths changing dynamically throughout the region. Some ligands appear to be very strong, with binding strengths comparable to siderophores (conditional stability constant $\left.\left(\log \mathrm{K}^{\text {cond }}\right)=11.1-13.9\right)$ [Buck et al., 2007; Bundy et al., 2014]. Siderophores are a class of iron ligands that are actively produced by marine microbes to bind ambient iron in a form that can only be taken up by organisms that possess specialized acquisition pathways. Early investigations to chemically characterize marine ligands from the CCS surveyed organic extracts from surface waters using colorometric assays. These studies determined that hydroxamate and catecholate functional groups, common iron binding moieties in siderophores, may be present in extracts of organic matter collected from surface seawater across the CCS [Macrellis et al., 2001]. Furthermore, metagenomic surveys from the Tara Oceans gene catalogue found 
homologues to both nonribosomal peptide synthases for amphibactin biosynthesis in the mesopelagic of the CCS (see Chapter 4). However, siderophores have not been directly identified in this environment.

Previous studies have also suggested that terrestrial or marine humic substances produced by the biotic and abiotic decay of organic matter may be a major component of iron ligands [Laglera and van den Berg, 2009; Laglera et al., 2011; Abualhaija et al., 2015; Bundy et al., 2015b]. New methods for investigating the molecular composition of these compounds are needed to constrain how they are made and consumed by the marine community.

In this study, I investigated the molecular composition of organic metal ligands across the California coast using liquid chromatography hyphenated with inductively coupled plasma mass spectrometry and electrospray ionization mass spectrometry (LC-ICPMS-ESIMS), focusing on the surface ocean and the benthic boundary along the continental shelf. The study's aim was to identify specific metal binding ligands and gain insight into their likely sources. These methods simultaneously generate information about other heteroatoms, including bromine and iodine, and preliminary investigations into the distribution of halogenated compounds is described in the appendices.

\subsection{Methods:}

\subsubsection{Materials and Reagents:}

High purity solvents and reagents were used throughout the study, including MilliQ water (18.2 $\left.\mathrm{MO} \mathrm{cm}, \mathrm{qH}_{2} \mathrm{O}\right)$, LCMS grade methanol $(\mathrm{MeOH})$ that was redistilled in a Polytetrafluoroethylene (PTFE) still, and LCMS grade ammonium formate (Optima, Fisher scientific). Polycarbonate bottles and PTFE tubing for sample collection and solid phase extraction (SPE) were soaked overnight in $0.1 \%$ detergent (Citranox), rinsed with $\mathrm{qH}_{2} \mathrm{O}$, and soaked for 1 day in $1 \mathrm{~N} \mathrm{HCl}$ (trace metal grade, Fisher Scientific) before a final $\mathrm{qH}_{2} \mathrm{O}$ rinse. Polypropylene tubes (Nunc, Thermo Scientific) used for sample concentration were washed with $\mathrm{qH}_{2} \mathrm{O}$ acidified to $\mathrm{pH} 2$ with hydrochloric acid ( $\mathrm{HCl}$, trace metal grade, Fisher Scientific) followed by a final rinse with $\mathrm{qH}_{2} \mathrm{O}$ 
and $\mathrm{MeOH}$ prior to use. A $50 \mu \mathrm{M}$ stock solution of cyanocobalamin (Sigma Aldrich) was prepared in $\mathrm{qH}_{2} \mathrm{O}$. An aqueous solution of iron citrate was prepared by combining $1 \mathrm{mM}$ sodium citrate dihydrate and $100 \mu \mathrm{M}$ iron (diluted from a 1000ppm iron reference standard solution, Fisher chemical).

\subsubsection{Sample collection:}

Samples were collected during the IRNBRU cruise along the CCS between Santa Barbara and the Oregon coast in 2014, an anomalous year in this region due to El Nino wind patterns leading to reduced upwelling (Fig. 5.1). As a result, the typical strong chemical gradients found in this region were dampened, and the 'transition' region between upwelling and oligotrophic conditions was greatly expanded. Surface water samples (20L volume) were collected while transiting using a trace metal clean GeoFish sampling system. Depth profile samples for organic extraction and analysis were collected using PTFE coated GO-Flo bottles deployed on a Kevlar hydroline [Bruland et al., 1979]. Hydrographic data and samples for nitrate analysis were collected using the $\mathrm{R} / \mathrm{V}$ Melville rosette system with conductivity, depth, temperature, fluorometer, dissolved oxygen, and beam transmission sensors. Nitrate concentrations were analyzed shipboard by flow injection analysis using standard spectrophotometric methods [Parsons et al., 1984]. Dissolved iron concentrations were determined either shipboard using a flow injection based analysis and spectrophotometric detection of iron chelated by N,Ndimethyl-p-phenylenediamine dihydrochloride (DPD) [Biller et al., 2013] or post-cruise using preconcentration with Nobias-chelate PA1 resin and quantification by ICPMS [Biller and Bruland, 2012].

Samples collected for iron ligand analysis by LC-ICPMS-ESIMS were filtered through $0.2 \mu \mathrm{m}$ Polyethersulfone capsule filters into 20L polycarbonate carboys, and were pumped through solid phase extraction resin columns $(1 \mathrm{~g}, \mathrm{ENV})$ at a rate of $15 \mathrm{~mL} / \mathrm{min}$ using a peristaltic pump equipped with polytetraflouroethylene (PTFE) and platinum-cured silicone tubing. Immediately before use, the columns were primed with $5 \mathrm{~mL} \mathrm{MeOH}$, rinsed with $10 \mathrm{~mL} \mathrm{pH} 2 \mathrm{qH}_{2} \mathrm{O}$ (acidified with trace metal grade $\mathrm{HCl}$ ), and conditioned with $10 \mathrm{~mL} \mathrm{qH}_{2} \mathrm{O}$. After extraction, the columns were rinsed with $10 \mathrm{~mL} \mathrm{qH}_{2} \mathrm{O}$ and frozen onboard. Prior to analysis, the samples were thawed, 
eluted with $10 \mathrm{~mL} \mathrm{MeOH}$ into $15 \mathrm{~mL}$ polypropylene centrifuge tubes, and concentrated in a vacuum rotary centrifuge until only residual water was remaining $(<200 \mu \mathrm{L})$. The sample was then transferred to a $1.5 \mathrm{~mL}$ microcentrifuge tube and brought to a final volume of $1 \mathrm{~mL}$ with $\mathrm{qH}_{2} \mathrm{O} .10 \mu \mathrm{L}$ of $10 \mu \mathrm{M}$ cyanocobalamin was added as an internal standard. Samples were then analyzed by LC-ICPMS and LC-ESIMS. To saturate unbound ligands and investigate total concentrations, a $100 \mu \mathrm{L}$ aliquot of each sample was also spiked with $10 \mu \mathrm{L}$ of iron citrate solution and analyzed.

\subsubsection{Zooplankton grazing:}

Zooplankton grazing studies were carried out twice during the IRNBRU cruise. Zooplankton were collected at night with a $200 \mu \mathrm{m}$ ring net from the upper $80 \mathrm{~m}$. Cod end contents were filtered through a $5 \mathrm{~mm}$ sieve to isolate larger zooplankton and individual copepods were handpicked for experiments. Phytoplankton within a $20-200 \mu \mathrm{m}$ size fraction were concentrated by filtration from $80 \mathrm{~L}$ of water collected by the GeoFish sampling system to a final volume of $500 \mathrm{~mL}$. Four treatments were incubated in triplicate $2.5 \mathrm{~L}$ polycarbonate bottles on the ship deck for 12-24 hours in the dark: (1) a filtered seawater control with no further treatment (2) filtered seawater with $75 \mathrm{~mL}$ of the concentrated phytoplankton stock (3) filtered seawater with 10 copepods added, and (4) filtered seawater with $75 \mathrm{~mL}$ of concentrated phytoplankton and 10 copepods added. For each treatment, $1.5 \mathrm{~L}$ from each replicate were combined $(4.5 \mathrm{~L}$ total) and were filtered and extracted as described above for LCMS analysis.

\subsubsection{Liquid chromatography:}

Chromatography was performed on a bioinert Dionex Ultimate 3000 HPLC system fitted with a polyetheretherketone (PEEK) c8 column $(3 \mu \mathrm{M}, 2.1 \times 100 \mathrm{~mm}$, Hamilton). Compounds were separated at a flow rate of $200 \mu \mathrm{L} / \mathrm{min}$ with a 20 minute gradient from $90 \%$ qH2O plus $5 \mathrm{mM}$ ammonium formate (A) and $10 \% \mathrm{MeOH}$ plus $5 \mathrm{mM}$ ammonium formate (B) to $90 \% \mathrm{~B}$, followed by isocratic elution at $90 \%$ B for 10 minutes. A post column flow splitter directed $50 \mu \mathrm{L} / \mathrm{min}$ to the mass spectrometer. 


\subsubsection{ICPMS analysis:}

Methods for ICPMS and ESIMS analyses were adapted from our previous study (Chapter 4). LC-ICPMS analysis was carried out with a quadrupole ICPMS (thermo icapQ) coupled to a bioinert liquid chromatography system (Dionex Ultimate 3000). Compounds were separated on a C8 column (Hamilton, 2.1x100mm, $3 \mu \mathrm{m}$ particle size) and polyetheretherketone (PEEK) tubing and connectors using a gradient from $90 \% 5 \mathrm{mM}$ aqueous ammonium formate (A) to $90 \% 5 \mathrm{mM}$ ammonium formate in distilled $\mathrm{MeOH}$ (B) over 20 minutes followed by isocratic elution at 90\% B for 10 minutes at a flow rate of $0.2 \mathrm{~mL} / \mathrm{min}$. A post column PEEK flow splitter directed $50 \mu \mathrm{L} / \mathrm{min}$ into the ICPMS or ESIMS.

Samples were analyzed by LC-ICPMS both with and without Fe addition to determine concentrations of both iron bound and total ligand concentrations. Isotopes monitored during ICPMS analysis included ${ }^{55} \mathrm{Mn},{ }^{56} \mathrm{Fe},{ }^{57} \mathrm{Fe},{ }^{59} \mathrm{Co},{ }^{60} \mathrm{Ni},{ }^{63} \mathrm{Cu},{ }^{66} \mathrm{Zn},{ }^{79} \mathrm{Br}$, and ${ }^{127} \mathrm{I}$ with integration times of 0.05 seconds. Oxygen gas was introduced to the plasma at $25 \mathrm{~mL} / \mathrm{min}$ to prevent the deposition of reduced organics on the cones. The ICPMS was equipped with platinum sampler and skimmer cones, a perfluoroalkoxy micronebulizer (PFA-ST, Elemental Scientific), and a cyclonic spray chamber cooled to $0^{\circ} \mathrm{C}$.. Measurements were made in kinetic energy discrimination mode with a He collision gas introduced at a rate of $4.2 \mathrm{~mL} / \mathrm{min}$ to remove $\mathrm{ArO}^{+}$ interferences on ${ }^{56} \mathrm{Fe}$.

To determine iron ligand concentrations, a standard curve of 12,25,50, and $100 \mathrm{nM}$ ferrioxamine E spiked with cyanocobalamin were analyzed. Integration of ICPMS signals and calculation of concentrations were carried out with in-house scripts written in $\mathrm{R}$ as described in chapter 4 . Total ligand concentrations were calculated by integrating the entire LCMS curve between 3 to 32 minutes with a blank subtraction and converting peak area to concentration based on the sensitivity for ferrioxamine E. Since the ICPMS sensitivity decreases as the organic content of the mobile phase increases $(40 \%$ decrease over the course of the run from $10-90 \% \mathrm{~B})$, this approach slightly underestimates the concentration of compounds that elute after ferrioxamine $\mathrm{E}$ (11.1 minutes). 


\subsubsection{ESIMS analysis:}

Samples were analyzed by LC-ESIMS using the same chromatography coupled to an Orbitrap Fusion Tribrid mass spectrometer equipped with a heated electrospray ionization source. The source parameters were set to a capillary voltage of $3500 \mathrm{~V}$, sheath, auxiliary and sweep gas flow rates of 12,6 , and 2 (arbitrary units), and ion transfer tube and vaporizer temperatures of $300^{\circ} \mathrm{C}$ and $75^{\circ} \mathrm{C}$. Scans were collected in positive mode with $450 \mathrm{~K}$ mass resolution. The most abundant ions in each scan were targeted for MS2 analysis using quadrupole isolation with a $1 \mathrm{~m} / \mathrm{z}$ mass window, an ion collection time of $25 \mathrm{~ms}$, a collision energy of $35 \%$ in HCD mode, and were detected on the low resolution ion trap mass analyzer.

LC-ESIMS data was analyzed in $\mathrm{R}$ after conversion to mzXML file format (MSconvert, proteowizard). A constant time offset was applied to align the retention time of the cyanocobalamin $[\mathrm{M}+\mathrm{H}]]^{2+}$ peak $(\mathrm{m} / \mathrm{z}=678)$ with the cyanocobalamin ${ }^{59} \mathrm{Co}$ peak in the LCICPMS chromatogram. The isotope pattern matching algorithm described previously [Boiteau and Repeta, 2015] was used to identify the masses of iron containing compounds by picking out $\mathrm{m} / \mathrm{z}$ and intensity features from each scan that match mass difference and abundance ratio of ${ }^{56} \mathrm{Fe}$ and ${ }^{54} \mathrm{Fe}$, and that appear as coherent peaks that match the retention time of the LC-ICPMS peaks (Appendix 5-1).

\subsection{Results:}

\subsubsection{Iron in wide shelf upwelling regions:}

Coastal upwelling along the CCS brings cold, salty water enriched in nutrients to the surface [Biller and Bruland, 2014]. In regions where this upwelled water passes over a shallow continental shelf, it picks up high concentrations of iron, manganese, cobalt and copper [Biller and Bruland, 2013]. As a result of these trace metal and nutrient additions, primary productivity rates soar in these diatom dominated wide shelf regions [Hutchins and Bruland, 1998; Bruland et al., 2001]. Sample profiles were collected from a wide shelf region located just north of San Francisco Bay (Fig. 5.1, station 2). 
The wide continental shelf station was characterized by high nitrate $(15.9 \mu \mathrm{M})$ and dissolved iron $(5 \mathrm{nM})$ at the surface, along with intense biological productivity within the mixed layer (chlorophyll a fluorescence of $10 \mu \mathrm{g} / \mathrm{L}$ ). Surface water temperatures were $10^{\circ} \mathrm{C}$ reflecting the recent upwelling of cold, salty deep water. A clear benthic iron source at this station is reflected in the high dissolved iron concentrations of $17.7 \mathrm{nM}$ near the sediment-water interface at $90 \mathrm{~m}$. There are also two plumes of suspended sediments that were detected as decreases in beam transmission centered at $60 \mathrm{~m}$ and $90 \mathrm{~m}$ (Fig. 5.2a).

Samples for ligand characterization were collected from six depths in the shallow continental shelf region. The resulting chromatograms show four main Fe features: (1) very low concentrations of discrete iron compounds in the surface mixed layer waters at 8 and $10 \mathrm{~min}$, (2) Two discrete peaks eluting at 19 and $21 \mathrm{~min}$, (Fig 5.2, labeled a, b) that were found at 60m and 90m depth, (3) a distinctive broad signal of chromatographically unresolved polar compounds eluting between 4-20 minutes, that increased gradually with depth, and (4) a broad signal eluting at $25 \mathrm{~min}$, found predominately in the surface ocean.

Major discrete compounds were identified by LC-ESIMS. The low abundance compound appearing in the surface samples at 12 minutes was identified as ferrioxamine B based on its retention time and a peak with the same retention time in the LC-ESIMS data at the ${ }^{56} \mathrm{Fe} \mathrm{m} / \mathrm{z}$ 614.273, although the ${ }^{54} \mathrm{Fe}$ isotopologue signal was masked by background interference at the same mass. Another compound was detected in the surface waters by ICPMS eluting at $8 \mathrm{~min}$, but was not detected as a distinctive mass in ESIMS. The two discrete compounds, A and B, were identified as the siderophores synechobactin C12 (also known as synechobactin A), and synechobactin $\mathrm{C} 14$. Monoisotopic masses for the $[\mathrm{M}+\mathrm{H}]{ }^{56} \mathrm{Fe}$ forms were found to be 614.261 and 642.292 respectively. MS2 fragmentation of these ions yielded diagnostic fragments for synechobactins, including neutral losses of $46.006\left(-\mathrm{CH}_{2} \mathrm{O}_{2}\right), 113.996\left(-\mathrm{C}_{4} \mathrm{H}_{2} \mathrm{O}_{4}\right), 130.027(-$ $\left.\mathrm{C}_{5} \mathrm{H}_{6} \mathrm{O}\right)$, and $156.006\left(-\mathrm{C}_{6} \mathrm{H}_{4} \mathrm{O}_{5}\right)$.

The broad ${ }^{56} \mathrm{Fe}$ signals observed in the chromatogram between 3-20 minutes (Fig. 5.2) represent a diverse suite of organic-iron complexes compounds that elute from the chromatography column over a wide range of retention times. These compounds likely correspond to the 
refractory carboxylic acid rich compounds that account for the majority of dissolved organic matter in the ocean [Hertkorn et al., 2015; Repeta, 2015] and have a reasonably strong affinity for complexing iron [Abdulla et al., 2010; Bundy et al., 2015a]. The benthic boundary appears to be the major source of these chromatographically unresolved polar iron ligands as well as dissolved iron. The question remains whether they are produced in situ by microbial processes in marine muds, or if they are transported from rivers, settle out of the water column by flocculation or sorption to particles, and are released when the sediment is resuspended. These compounds are less abundant in the surface mixed layer, suggesting that they are removed or altered by biological activity or photochemistry.

The siderophores synechobactin $\mathrm{C} 12$ and $\mathrm{C} 14$ also appear to be supplied by the mud belt. These compounds were only present in samples that were collected from within nepheloid layers at $60 \mathrm{~m}$ and $90 \mathrm{~m}$ along the wide shelf station. Concentrations were high (18pM and $12 \mathrm{pM})$, and the synechobactins were saturated with iron, which is consistent with the high ambient iron concentrations at these depths. Since these compounds are not found elsewhere across the CCS away from this resuspended sedimentary source, it seems that the compounds are rapidly removed from the water column. Synechobactins are susceptible to biological consumption as well as photooxidation of the alpha-hydroxy carboxylate group when iron bound [Barbeau et al., 2003; Ito and Butler, 2005]. While these resuspended sediments appear to be a source of synechobactins at 60 and $90 \mathrm{~m}$, no increase in total dissolved iron is observed at these depths. Instead, high concentrations of dissolved iron and unresolved organic ligands appear to be sourced from the sediment-water interface. This suggests that multiple processes are adding iron ligands of different composition and reactivity to the water column from the benthic boundary.

\subsubsection{Iron in the narrow shelf upwelling region:}

In regions of the CCS with narrow continental shelves, upwelled water supplies less iron to the surface compared to the wide shelf regions because the water does not pick up material from shallow sediments [Bruland et al., 2008; Biller et al., 2013; Bundy et al., 2014]. As a result, iron is quickly depleted from these waters, resulting in less expansive diatom blooms and higher concentrations of residual nitrate [King et al., 2012a; Biller and Bruland, 2014]. 
During the IRNBRU cruise, two samples were collected for ligand characterization from a region of recent coastal upwelling along the narrow shelf region off of the Oregon coast (Fig. 5.3, Station 28). Surface temperature, salinity, and nitrate concentrations were similar to those along the wide shelf region. However, iron concentrations were much lower (0.3-0.4 $\mathrm{nM})$, and the resulting phytoplankton bloom was far less intense. In this region, only chromatographically unresolved ligands were observed. Concentrations of these ligands were determined by integrating the total $56 \mathrm{Fe}$ signal over time relative to an internal standard (cyanocobalamin) and converting this area to concentration based on a standard curve. These ligands appear to be more abundant just below the deep chlorophyll maximum compared to near the surface. Interestingly, the deep water appears to contain much higher concentrations of excess ligand than the surface waters, suggesting that they is a source for these compounds at depth or in sediments and potentially a sink in the surface mixed layer, possibly including consumption by microbes or degradation by photochemistry.

\subsubsection{Iron in the Santa Barbara basin:}

The Santa Barbara basin is a region where elevated sills restrict lateral water advection below $475 \mathrm{~m}$. High organic matter fluxes from productive surface waters result in heavy $\mathrm{O}_{2}$ depletion within the deep water, accompanied by high concentrations of sediment derived reduced Fe(II) [John et al., 2012], and high dissolved organic matter concentrations (200-300 $\mu \mathrm{M})$ [Chen et al., 1993]. While high iron concentrations within the anoxic waters at the Santa Barbara basin have been previously observed [John et al., 2012], most of this iron is removed rapidly just above the anoxic zone, consistent with fast oxidation and precipitation as $\mathrm{Fe}(\mathrm{III})$ [Rose and Waite, 2003]. However, a substantial portion escapes this removal process. Estimates from iron isotope measurements suggest that the isotopically light iron released from reducing sediments may account for up to $4-12 \%$ of the iron in the world ocean [John et al., 2012]. Stabilization by organic ligands may determine how much of this dissolved iron remains dissolved in oxic waters rather than precipitating out as iron oxyhydroxides. Alternatively, this iron could potentially form stable oxyhydroxide colloids that remain suspended in the water column for extended periods of time. About $20-80 \%$ of the iron in seawater is found in the colloidal size fraction, and 
much of this iron is inert to chemical exchange with added iron ligands within 1-12 hr timescales [Cullen et al., 2006; Fitzsimmons et al., 2014]. It is currently unclear whether this is because the iron is present as inert inorganic phases or packaged within macromolecules or biological structures (e.g. vesicles or viruses) with slow exchange kinetics.

In this study, samples for ligand analysis were collected from the Santa Barbara basin at five depths (Fig. 5.4, Station 29). The surface waters at this site had very low nitrate $(<0.02 \mu \mathrm{M})$ and moderate iron $(4.6 \mathrm{nM})$ concentrations. There was a maximum in chlorophyll florescence at $20 \mathrm{~m}$ just below the mixed layer depth at $18 \mathrm{~m}$, and a gradual decrease in $\mathrm{O}_{2}$ with depth. Oxygen was depleted by $500 \mathrm{~m}$, marked by the onset of nitrate reduction and a gradual decline in nitrate with depth. Iron concentrations were elevated in the anoxic zone and increased rapidly with depth, reaching concentrations above 50nM near the sediment interface.

While no discrete iron binding compounds were detected by LC-ICPMS at this site, chromatographically unresolved ligands were present at all depths. Samples collected from within the anoxic region exhibited the same broad signal between 4-20 minutes that was observed at depth in the wide shelf region. Addition of extra iron to these samples to saturate unbound ligands resulted in a nearly identical chromatogram compared to the unamend samples, indicating that there were no iron-free ligands present. These ligands may prevent the precipitation of dissolved iron under oxic conditions, which would induce the oxidation of soluble $\mathrm{Fe}(\mathrm{II})$ to $\mathrm{Fe}(\mathrm{III})$ and favor precipitation as oxyhydroxides unless the iron were stabilized as an organic complex. These unresolved ligands rapidly decrease in abundance away from the benthic boundary, suggesting a sedimentary source.

\subsubsection{Transition region:}

The transition region is characterized by intermediate surface water temperatures and a wide range of nutrient and metal concentrations (Fig 5.5). The high metal concentrations drop quickly once away from the coast to values of $0.25-0.03 \mathrm{nM}$ in the surface ocean. At these low metal concentrations the autotrophic community shifts from diatoms to unknown eukaryotic autotrophs [King et al., 2012b]. In regions where nitrate values remain high $\left([\mathrm{dFe}]:\left[\mathrm{NO}_{3}{ }^{-}\right]<0.2 \mathrm{nmol} / \mu \mathrm{mol}\right)$, 
iron amendments increase productivity, suggesting iron limitation to autotrophic growth [King et al., 2012b].

In this transition region, defined by warm surface waters $\left(>12^{\circ} \mathrm{C}\right)$, nonpolar iron binding compounds with retention times between 19-24 minutes were detected in most of the samples collected from the surface waters or deep chlorophyll maximum in this region (Fig. 5.6). The most abundant and widespread compounds, eluting at 19.7, 20.5, 21.1, 21.9, and 22.2 minutes were identified as amphibactins S, D E, H, and I (m/z 883.399, 885.415, 911.430, 913.446, and 939.462). The MS2 spectra of these compounds showed diagnostic neutral losses of $\mathrm{m} / \mathrm{z} 218$ $\left(\mathrm{C}_{8} \mathrm{H}_{14} \mathrm{~N}_{2} \mathrm{O}_{5}\right), 277\left(\mathrm{C}_{10} \mathrm{H}_{19} \mathrm{~N}_{3} \mathrm{O}_{6}\right)$, and $305\left(\mathrm{C}_{11} \mathrm{H}_{19} \mathrm{~N}_{3} \mathrm{O}_{7}\right)$. The most abundant amphibactins were consistently S, E, and I, which all have a single unsaturation in the fatty acid tail. Another compound with $709.371 \mathrm{~m} / \mathrm{z}$ was frequently observed at $22.9 \mathrm{~min}$. All of these compounds were also found in the HNLC region of the eastern tropical south Pacific Ocean during the GEOTRACES EPZT cruise (Chapter 4).

With discrete sampling in the California coast, the spatial diversity of these compounds can be seen. The amphibactins are widespread, appearing in 19 out of 23 samples collected from the transition region. The unknown $709 \mathrm{~m} / \mathrm{z}$ compound appears in seven of these samples. The source of the $709 \mathrm{~m} / \mathrm{z}$ compound appears to be distinct from the amphibactin source, since this compound appears at the highest concentrations in samples where amphibactin concentrations are low, primarily near the Oregon coast.

The range of amphibactin concentrations observed throughout this region suggests complex controls on their distribution. Some insight can be found by observing amphibactin concentrations within the surface and DCM of the eddies. We sampled the center of two cyclonic eddies (stations 9 and 15), and one anticyclonic eddy (station 12). The cold-core cyclonic eddies are composed of salty upwelled water originating from the coast. Within the cyclonic eddies wind-driven mixing or curl-induced upwelling supplies macronutrients to the surface from the shallow nutricline. The eastern eddy was composed of recently upwelled water and retains nitrate stocks, although iron was depleted. In this region, amphibactins were found at high concentrations in the surface $(7 \mathrm{pM})$, and lower concentrations at the DCM (3pM). Surface 
waters across this eddie were sampled again several days later, and revealed fine spatial scale variability, with higher concentrations of the unknown $709 \mathrm{~m} / \mathrm{z}$ compound found within the core of the eddy and amphibactins dominating closer to the edges (Fig. 5.7). These differences are likely attributed to differences in microbial community composition that arise within these dynamic eddy features.

Further offshore, the westward eddy was composed of warmer water that had spent more time at the surface and was significantly depleted in both nitrate and iron at the surface. Here, amphibactin concentrations were lower $(4 \mathrm{pM}$ at the surface and $0.6 \mathrm{pM}$ at the DCM). These lower amphibactin concentrations may have resulted from the change in productivity associated with the transition towards a macronutrient limited oligotrophic community. There were also potentially differences in the chemistry or seed populations of the initial water masses that formed these two eddies. In the future, Lagrangian studies of an upwelled water mass along the coast would provide insight into the timing of amphibactin production.

The anticyclonic eddy was characterized by warm, lower salinity water that likely originated from the California current, and a deeper nutricline. Amphibactin concentrations were low in the nitrate and iron deplete surface waters at this site $(1.4 \mathrm{pM})$. Surprisingly, amphibactins were observed at high concentrations $(8.5 \mathrm{pM})$ in the deep chlorophyll maximum at $48 \mathrm{~m}$ just above the nutricline. It is possible that the biological community at this depth was similar to the community in the surface of the cyclonic eddies. Alternatively, these compounds may persist for some time in the water column and could be relics of previous biological conditions.

Amphibactins were also found at low concentrations (3pM) in a sample collected from the deep ocean at $1500 \mathrm{~m}$. Elevated concentrations of particulate Fe $(1 \mathrm{nM})$ and $\mathrm{Al}(7 \mathrm{nM})$ at this depth suggest a strong influence of material from the continental slope. The amphibactins found in the deep sample may have been produced in situ as microbes degrade particulate organic matter that sank from the surface. Alternatively, they may have been sourced from continental slope sediments. Further studies are needed to investigate these potential sources as well as the persistence of these compounds in the deep ocean. 


\subsubsection{Grazing:}

Zooplankton grazing is a major recycling pathway for iron in the euphotic zone. When grazers feed on phytoplankton, the iron contents of their prey is either processed through the gut of the grazer where they may undergo chemical transformations [Barbeau et al., 1996], or it is released directly into the surrounding water. Furthermore, previous studies have demonstrated an increase in strong iron binding ligand concentrations that scaled proportionally to the chlorophyll consumed by zooplankton grazers [Sato et al., 2007]. The release of these ligands can potentially impact the bioavailability of this iron to certain classes of phytoplankton. Yet nothing is known about the identity of these ligands or the iron forms that are released to the dissolved iron pool by zooplankton grazing.

Grazing incubation experiments were performed on the IRNBRU cruise to investigate these iron regeneration processes. For this experiment, filtered surface seawater was inoculated with (a) nothing (control treatment) (b) phytoplankton collected from a net tow (phytoplankton treatment) (c) copepod grazers (zooplankton treatment), and (d) phytoplankton and copepods (grazing treatment). The treatments were incubated for 12-24 hrs.

In both experiments, the phytoplankton, zooplankton, and grazing treatments exhibited distinct iron peaks in the LC-ICPMS chromatograms (Fig. 5.8). These compounds were more abundant in the zooplankton and grazing treatments than in the phytoplankton treatment. The major compound eluting at 9.1 minutes was identified as the siderophore ferrioxamine G. Other major compounds, eluting at 19.7, 20.5, 20.6, 21.3, and 22.0 minutes were found to have ${ }^{56} \mathrm{Fe}$ monoisotopic $[\mathrm{M}+\mathrm{H}]^{+}$masses of $876,902,854,884$, and $882 \mathrm{~m} / \mathrm{z}$ respectively. These masses do not appear in the Chelomex database of 367 compounds. These compounds have a common $\mathrm{MS}^{2}$ fragment $(295.22 \mathrm{~m} / \mathrm{z})$, and neutral loss $(497.17 \mathrm{~m} / \mathrm{z})$, suggesting that they may be structurally related to each other.

Another peak, eluting at 19 minutes is significantly more abundant in the grazing treatment than in the other two treatments. This compound was found to have a ${ }^{56} \mathrm{Fe}$ monoisotopic $[\mathrm{M}+\mathrm{H}]^{+}$ 
mass of $968 \mathrm{~m} / \mathrm{z}$, which also has no match within the siderophore database. This compound exhibited major fragments of $306,334,351,393$, and $419 \mathrm{~m} / \mathrm{z}$.

In the copper LC-ICPMS chromatogram from the grazer experiment, a major compound eluting at 21.1 minutes was detected in both the zooplankton and grazing treatments, at roughly similar concentrations. While the mass of this compound was not determined, its presence suggests that zooplankton may be a source of metabolites that play a role in copper cycling or detoxification.

\subsection{Discussion:}

\subsubsection{Unresolved carbon compounds from the deep:}

One of the unexpected results from the survey of organic ligands across the CCS was the consistency of chromatographically unresolved dissolved organic matter (DOM) in samples collected from deep water. The samples collected from upwelling stations below the photic zone (2 and 28), from the anoxic region of the Santa Barbara Basin, and from the $1500 \mathrm{~m}$ sample collected from stations 12 all have a broad chromatographic feature from 4-20 minutes with a maximum at 8 minutes. It is likely that these unresolved organic compounds represent a common class of refractory organic matter that are produced in deep water or sediments as a result of organic matter degradation. These compounds are good candidates for the benthic boundary sourced ligands that have been previously reported by electrochemical studies from the California coast. Bundy et. al. 2014 found that moderately strong ligands were released from shallow mudflats along the continental shelf near San Francisco Bay. These ligands had conditional stability constants ( $\log \mathrm{K}_{\mathrm{FeL}, \mathrm{L}}^{\mathrm{cond}}$ ) ranging from 11.2-11.9, similar to those reported for humic substances. A portion of this material (estimated at 3-18\%) is potentially composed of terrestrially derived humic substances, as determined by direct measurements by adsorptive cathodic stripping voltammetry [Bundy et al., 2014, 2015b].

While the origin of iron binding unresolved DOM remains uncertain, there are several scenarios that are consistent with these results. (1) It is possible that these ligands are terrestrially derived. Dissolved terrestrial organic matter tends to flocculate when fresh water is mixed with high 
salinity ocean water [Boyle et al., 1977]. Upon flocculation, iron and strong iron binding ligands are lost from the water column into the sediments [Bundy et al., 2015a]. Over time, these compounds may re-dissolve and diffuse into the overlying deep water. If this is the primary source of iron ligands in the CCS, sedimentary sources close to river mouths or groundwater discharge would be expected to be more significant sources of metal ligands compared to sites further offshore. (2) Alternatively, the deep unresolved DOM might be produced by the degradation of organic matter of marine origin in the water column or within sediments. This explanation is consistent with the finding of high concentrations in the deep waters of the Santa Barbara basin, where most of the organic carbon is supplied from sinking marine biomass (estimates based on $\delta^{13} \mathrm{C}$ of particulate organic carbon in nearby sediments suggest that $\sim 17 \%$ is terrestrially derived). One remaining question is whether the ligands observed in the deep $1500 \mathrm{~m}$ sample from station 12 are produced within the water column or released from margin sediments and transported. Since these ligands are thought to regulate the amount of iron that makes it to the surface ocean from sediments [Buck et al., 2007], environmental changes that impact the ligand source will likely have important feedbacks on biological carbon uptake in the surface of the upwelling zone.

Sediments and deep water also exhibited elevated levels of unresolved DOM complexed with other trace metals. Similar features were also seen for $\mathrm{Zn}$ at all of these sites, and for $\mathrm{Cu}$ at all sites except for the Santa Barbara Basin. Intriguingly, no increase in $\mathrm{Cu}$ associated with unresolved DOM is observed from the sediments of the Santa Barbara Basin, though this result is consistent with anoxic sediments (unpublished data). The observation that unresolved DOM apparently binds multiple metals raises the question of whether these metals compete for the same binding sites, or if different components of unresolved DOM have a selective preference for certain metals.

\subsubsection{Siderophore distributions:}

Both known and novel siderophores were found at many locations throughout the CCS, reflecting microbial efforts to compete for iron under different nutrient regimes. The same siderophores that were observed in surface waters of the eastern tropical South Pacific were 
found in the CCS surface waters, and their distribution patterns are similar. In the previous study on the eastern tropical South Pacific (chapter 4), integrated surface samples were collected over wide regions to provide a zonal average siderophore composition. In this chapter, discrete samples were collected and analyzed from the CCS, providing an opportunity to evaluate the fine scale distribution of siderophores across multiple depths.

In the wide continental shelf region, low concentrations of ferrioxamine B were detected only within the surface mixed layer. Likewise, ferrioxamine B was found predominantly in the microplankton dominated coastal region of the eastern tropical South Pacific. The production of ferrioxamines are widespread across bacterial lineages including the gram-positive Streptomyces and Actinomycetes, and numerous gram-positive Gammaproteobacteria [Mucha et al., 1999; Martinez et al., 2001]. Furthermore, oceanographic surveys have also found the related compounds ferrioxamine G and E in the Baltic Sea [Mucha et al., 1999], the Atlantic Ocean [Mawji et al., 2008], and the oligotrophic North Pacific at Station Aloha (Boiteau, unpublished), and even in the grazing incubation study presented in this chapter. Thus, this class of siderophore appears to be widespread throughout the euphotic zone in areas where iron is not the limiting nutrient for overall primary productivity.

In regions where iron was depleted, amphibactins were the most common and abundant siderophore detected. Again, this mirrors the observation from the preceding chapter that these compounds were predominantly associated with the iron starved waters of the eastern tropical South Pacific. In the CCS, these compounds were found not only at the surface but also the deep chlorophyll maximum. Amphibactins were also detected within the California current deep water sample collected at $1500 \mathrm{~m}$. Since they were not detected in other deep samples from the wide continental shelf or from the Santa Barbara Basin, it appears that this is a localized signal, possibly generated by amphibactin producing microbes feeding on sinking particles from the productive overlying waters. The only known producers of amphibactin are Gammaproteobacteria, including Alcanivorax borkumensis SK2 and numerous vibrio strains (as discussed in Chapter 4), which release these compounds when iron concentrations are low. Thus, amphibactins may be primarily associated with opportunistic heterotrophs that regenerate iron from decaying organic matter. 
The unknown compound with m/z 709 was also previously detected in the South Pacific ocean, There, it co-occurred with amphibactins in the HNLC region. The exact mass of this ion matches a molecular formula of $\mathrm{C}_{33} \mathrm{H}_{59} \mathrm{~N}_{5} \mathrm{O}_{8} \mathrm{Fe}^{+}$. The fragmentation pattern of this compound is not similar to that of amphibactins (Fig. A5.1-6), and it is potentially a novel amphiphilic siderophore based on its late retention time and high carbon content. In this study, differences were observed in the relative abundance of the amphibactins and the unknown siderophore, suggesting that they are produced independently.

This study is also the first to identify synechobactins in the marine environment. Synechobactins are known to be produced by the mud-dwelling marine cyanobacteria Synechococcus sp. PCC 7002 [Ito and Butler, 2005; Boiteau and Repeta, 2015] and the nitrogen fixing cyanobacteria Anabaena variabilis [Deicke et al., 2014]. Based on the select regions where we find these compounds, it appears that these compounds are actively produced within the marine muds (either on the seafloor or within the resuspended water column), and that they are released when sediment/porewaters are resuspended. Further studies could confirm this by measuring these compounds within mud samples on the sea floor. Interestingly, synechobactins are a mixed-mode siderophore containing both hydroxamate and citrate iron binding groups. Due to their photolability, it may be no coincidence that these compounds are only found in marine muds where light levels are low.

One of the notable results from this survey is the lack of diversity of compounds appearing in the surface ocean in this study and others. Indeed, both ferrioxamines and amphibactins were also the major siderophores identified in carbon amended seawater incubation experiments by Gledhill and others performed across the Atlantic Ocean [McCormack et al., 2003; Gledhill et al., 2004; Mawji et al., 2008, 2011]. Yet many other siderophores have been isolated by marine bacteria in cultures which have yet to be observed in the environment, including marinobactins [Martinez and Butler, 2007], aquachelins[Martinez et al., 2000], loihichelins [Homann et al., 2009], ochrobactins [Martin et al., 2006], vibrioferrin [Amin et al., 2009], anguibactin [Jalal et al., 1989], vanchrobactin [Sandy et al., 2010], petrobactin [Barbeau et al., 2002], and 
moanachelins [Gauglitz and Butler, 2013]. Why have these compounds not appeared in any marine surveys thus far?

One potential explanation is that the extraction procedure provides a selective window into siderophore composition. Some siderophores, such as the citrate based compound vibrioferrin, are not retained by the SPE columns used in this study (unpublished results). Other compounds may be retained too strongly by the column to elute with methanol and acetonitrile. However, many known marine siderophores are efficiently retained and eluted from these columns, including schizokinin, aerobactin, anguibactin and marinobactin [Martinez et al., 2001; Ito and Butler, 2005; Zane et al., 2014; Boiteau, unpublished data].

Another possibility is that these other siderophores are degraded or consumed more rapidly than the ferrioxamine and amphibactin siderophores, either in the water column or during extraction or sample processing. For instance, citrate, $\alpha$-hydroxy acid, and catechol based siderophores are photolabile [Barbeau et al., 2001, 2003; Grabo et al., 2014] and may be lost quickly in the surface ocean or else their production might be selected against due to their reactivity. More detailed tests on the extraction efficiency of other siderophores from seawater would be needed to explicitly rule out this possibility. A related question is whether the siderophores that we observe represent molecules that are actively produced and used at the location where they were collected, or if they are long-lived compounds that persist in the water column. However, the ability to utilize iron associated with siderophores should provide a competitive advantage in iron starved environments, where we observe the highest siderophore concentrations. Under these conditions, positive selection pressure should increase the success of microbes that efficiently take up siderophores and lower standing stocks in the absence of increased production rates. Further studies on the uptake and turnover rates of these compounds within the water column are needed to test this hypothesis.

A final explanation is that amphibactin and ferrioxamine production is genuinely more common than many other siderophores in the open surface ocean where most of these surveys have taken place. Perhaps siderophores are very strongly associated with particular environmental niches. In this case, other siderophores will likely be discovered under environmental conditions that have 
yet to be explored. The results of this study support such a hypothesis. For example, synechobactins were found for the first time in this study associated within resuspended mud layers, and potential novel siderophores were found associated with zooplankton hosts (Table 5.2). As more environments are surveyed, more complete patterns of siderophore production will likely emerge.

But then what drives the distribution of different siderophores? In theory, it could stem from convergent evolution, where microbes happen to evolve different siderophore mediated strategies for the same function of iron acquisition. However, the ability to produce a specific siderophore does not appear to be linked to certain bacterial lineages, but are perhaps rather linked to ecological niches. Many bacterial genera are capable of producing multiple different types of siderophores, indicating that horizontal gene transfer is common for siderophore production and uptake pathways. For instance, marine Vibrio isolates have been found to produce vibrioferrin, anguibactin, aerobactin, amphi-enterobactin, and amphibactins [Jalal et al., 1989; Haygood et al., 1993; Vraspir et al., 2011; Cordero et al., 2012; Zane et al., 2014]. Furthermore, often a single strain possesses genes for the production of more than one siderophore, or for the uptake of a siderophore that the strain cannot produce [Cordero et al., 2012]. Together, these findings suggest that intense environmental selection pressure forces bacteria to regularly adapt to specific ecological niches and environmental nutrient regimes by acquiring the most successful siderophore mediated uptake strategies from their neighbors. Determining the ecological advantage of using a particular siderophore over others will require more detailed studies on the chemical properties of the siderophores and the lifestyles of the producers and consumers of these siderophores. Many factors are likely important, including binding strength, diffusion rates, energy/nutrient cost, and compatibility with host organisms or symbionts (e.g. phytoplankton or zooplankton).[Völker and Wolf-Gladrow, 1999; Vraspir and Butler, 2009; D'Onofrio et al., 2010; Hibbing et al., 2010] As a first step, genes involved in the complete biosynthetic and uptake pathway for these compounds must be determined. These genes can then be identified in single cell genomes isolated from the regions where these compounds are found in order to determine which organisms can produce them. Secondly, uptake studies with siderophores that are found in the marine environment will help to elucidate which members of the microbial community are able to utilize siderophore bound iron. 
Numerous studies have suggested that ferrioxamine B associated ${ }^{55} \mathrm{Fe}$ uptake rates vary widely for different phytoplankton [Hutchins et al., 1999a, 1999b; Shaked et al., 2005], and similar studies can be conducted with other environmentally relevant siderophores.

\subsubsection{What is retained and what is not?:}

The analytical window of mass spectrometry based investigations of marine ligand composition is largely determined by the solid phase extraction method used to concentrate and desalt the samples, and the chromatography that is used to separate the compounds. In this study, these parameters were carefully chosen to avoid sample manipulation that may degrade organic ligands or impact iron speciation (e.g. pH change). Polystyrene divinylbenzene resins such as ENV and PPL are commonly used for solid phase extraction of organic matter and have been previously shown to efficiently retain iron and iron binding compounds from natural waters at neutral pH [Macrellis et al., 2001; McCormack et al., 2003; Waska et al., 2015]. While the primary goal of this study was to structurally characterize iron ligands from different regions across the CCS, the next important aim is to determine their contribution to the dissolved iron pool. Additional samples were collected during the IRNBRU cruise to evaluate metal extraction efficiencies as well as alternative extraction methods, providing an opportunity to evaluate the quantitative recovery of dissolved iron and biomolecules using the extraction and analytical methods described in this study. Comparing the total dissolved iron concentrations to the concentrations of iron detected by LC-ICPMS reveals that the latter method only captures a small portion (2-5\%) in regions where iron concentrations are high (e.g. wide shelf and Santa Barbara Basin, Fig. 5.2, 5.4) compared to up to $30 \%$ in regions where dissolved Fe is low (e.g. narrow shelf, Fig. 5.3). Furthermore, total ligand concentrations in the surface ocean are often much higher $(1-2 \mathrm{nM})$. This raises the important question of what iron ligands were not observed during our analyses.

Measurements of dissolved iron in seawater before and after extraction reveal that $20-80 \%$ of dissolved iron is retained by the column (Fig. 5.9), consistent with other studies [Waska et al., 2015]. In some cases, the breakthrough of dissolved iron is likely due to poor retention of polar iron species. Citrate or ethylenediaminetetraacetate (EDTA) bound iron, for example, is not 
retained by the ENV resin used in this study. In other cases, it may be due to saturation of binding sites, especially in the coastal ocean where organic matter concentrations can be high. Breakthrough and column saturation was evaluated by extracting several seawater samples through two extraction columns stacked in tandem so that the flow through from the first column passes through a second extraction column (Fig. 5.10). These studies showed that most of the nonpolar compound eluting after 12 minutes, including amphibactins, were efficiently captured by the primary column. However, more polar iron binding compounds tended to break through and accumulate on the second column. Overall ligand concentrations from the second column were $21-22 \%$ relative to what was captured on the primary column. Finally, there is likely a significant portion of even more polar iron species that is simply not retained by either SPE column, possibly complexed by small metabolites or acidic polysaccharides [Hassler et al., 2011, 2014].

The metal binding compounds that are detected and quantified by the LC-ICPMS method described here are within the soluble fraction of dissolved iron/ligands $(<10 \mathrm{kDa})$. The LCICPMS chromatogram of SPE extracted organic compounds from filtered seawater $(0.2 \mu \mathrm{m}$ cutoff) and tangential flow filtered seawater (Pellicon, $<10 \mathrm{kDa}$ cutoff) are nearly identical (Fig. 5.10). Thus, it appears that colloidal iron is not detected by the LC-ICPMS method despite the fact that it makes up a significant portion of total dissolved iron in the CCS $(35-85 \%, \mathrm{~J}$. Fitzsimmons, unpublished data). This is likely explained by the small pore size of the ENV resin used during extraction $(45 \mathrm{~nm})$ and the C8 column used for separation $(11 \mathrm{~nm})$, which is not ideal for retaining and eluting large colloidal compounds. Currently, it is uncertain whether colloidal compounds are not retained by the SPE column or if they are retained but not eluted, perhaps getting caught in the narrow pores of the resin beads.

From the mass balance analysis described above, it appears that much of the iron that is retained by the solid phase extraction column $(20-80 \%$ of total $\mathrm{dFe})$ is retained too strongly by the SPE or chromatography column to elute with the solvents used in this study. There is precedence for marine dissolved iron to have a high affinity for polymer surfaces. For example, loss of iron to the walls of plastic bottles is common [Fitzsimmons and Boyle, 2012], and in ultrafiltration studies only $\sim 60 \%$ of the dissolved iron passing through the filter is typically recovered. There 
are several possible explanations as to why this iron is retained so strongly: (1) it is trapped in narrow pores as colloidal iron (2) it forms precipitates that nucleate on the plastic surfaces, or (3) it is associated with non-polar organic ligands that have high affinity for non-polar materials. Elution of the SPE columns with acetonitrile after the $\mathrm{MeOH}$ elution did recover up to $25 \%$ of additional organically bound iron, including amphibactins. There is evidence that rinsing columns with acidified water before the methanol elution step increases iron yields, potentially due to the dissolution of colloidal or precipitated iron forms, or to protonation of immobilized organic chelates (interestingly, this treatment does not significantly impact organic carbon yields) [Waska et al., 2015]. Another step where iron can be lost is during sample preconcentration. In many of the samples from the CCS, a precipitate formed in the final concentrate due to over saturation once the organic matter was concentrated 20,000x. It is likely that this precipitate contains at least a portion of the 'missing' iron.

One major factor that determines which compounds are isolated from seawater during extraction is the composition of the solid phase resin. We did replicate extractions of seawater from two locations using multiple resins to investigate the effect of the resin on the compounds that are extracted. Four solid phases were tested, two polystyrene based resins (ENV and PPL), one functionalized silica based resin (c18), and one activated charcoal resin (Envi-carb). The results of analyzing the same samples after extraction with these four different resins show different affinities for organic compounds (Fig. 5.11). Amphibactins were effectively extracted by ENV, PPL, and c18 phases, with the greatest recovery observed for the ENV resin. The activated charcoal resin tended to capture low concentrations of more polar compounds that were not captured by the other resins. Thus, using multiple resin phases in line may improve the overall recover of organic iron binding ligands from seawater in future studies.

Overall, the study present here provides a first window into the identity of compounds that bind iron. Due to the potential for incomplete recovery of iron species from the extraction column, the estimates of siderophore and iron ligand concentration described here are conservative lower bounds. More importantly, these results reveal specific compounds that are involved in biogeochemical iron cycling within the CCS, and thus enable more detailed studies to better constrain their concentrations and cycling rate. Future work will focus on optimizing the 
extraction and elution methods for the siderophores that were characterized during this study, and on varying the seawater extraction and elution methods in order to obtain new analytical windows that capture other portions of the dissolved iron pool. In addition to using multiple resins to extract a greater portion of iron ligands from seawater, acidifying the sample to protonate charged ligands might also improve the recovery of more polar compounds.

\subsection{Conclusions:}

This study reveals the diversity of iron ligands across the CCS. Siderophores make up a dynamic component of the iron ligand pool, with ferrioxamines B, E, and G appearing in nutrient rich waters and grazing incubation experiments, and amphibactins and an unknown iron ligand with $\mathrm{m} / \mathrm{z}$ of 709 detected throughout the low-iron surface waters. For the first time, synechobactins were detected in the marine environment, associated with resuspended mud layers along the continental shelf, suggesting that siderophores play a potential role for iron remobilization and cycling in sediments. Finally, zooplankton grazing was a source of an unknown iron compound with $\mathrm{m} / \mathrm{z}$ 968. These results confirm previous studies suggesting that siderophore production is widespread and heterogeneous [Mawji et al., 2008, 2011], and provide new important targets for future studies on biological iron uptake rates, iron remineralization/scavenging rates, and ligand turnover rates.

\subsection{Acknowledgements:}

Dissolved Fe measurements and sea surface height plots were generated by Claire Parker, UCSC. Dissolved nitrate measurements were made by Tyler Coale, UCSD. Special thanks to Serena Dao, Jessica Fitzsimmons, and Geoffrey Smith for assistance with sample collection. Thanks to the chief scientist of the IRNBRU cruise, Ken Bruland, and to the scientists and crew of the R/V Melville.

\subsection{References:}

Abdulla, H. A. N., E. C. Minor, R. F. Dias, and P. G. Hatcher (2010), Changes in the compound classes of dissolved organic matter along an estuarine transect: A study using FTIR and 13C NMR, Geochim. Cosmochim. Acta, 74(13), 3815-3838, doi:10.1016/j.gca.2010.04.006. 
Abualhaija, M. M., H. Whitby, and C. M. G. van den Berg (2015), Competition between copper and iron for humic ligands in estuarine waters, Mar. Chem., 172, 46-56, doi:10.1016/j.marchem.2015.03.010.

Agarwal, V., and B. S. Moore (2014), Enzymatic Synthesis of Polybrominated Dioxins from the Marine Environment., ACS Chem. Biol., 7-11, doi:10.1021/cb5004338.

Agarwal, V., A. a El Gamal, K. Yamanaka, D. Poth, R. D. Kersten, M. Schorn, E. E. Allen, and B. S. Moore (2014), Biosynthesis of polybrominated aromatic organic compounds by marine bacteria., Nat. Chem. Biol., 10(8), 640-647, doi:10.1038/nchembio.1564.

Agarwal, V., J. Li, I. Rahman, M. Borgen, L. I. Aluwihare, J. S. Biggs, V. J. Paul, and B. S. Moore (2015), Complexity of naturally produced polybrominated diphenyl ethers revealed via mass spectrometry., Environ. Sci. Technol., doi:10.1021/es505440j.

Amin, S. A., D. H. Green, F. C. Küpper, and C. J. Carrano (2009), Vibrioferrin, an unusual marine siderophore: iron binding, photochemistry, and biological implications., Inorg. Chem., 48(23), 11451-8, doi:10.1021/ic9016883.

Amin, S. A., M. S. Parker, and E. V. Armbrust (2012), Interactions between Diatoms and Bacteria, Microbiol. Mol. Biol. Rev., 76(3), 667-684, doi:10.1128/MMBR.00007-12.

Barbeau, K., J. W. Moffett, D. A. Caron, P. L. Croot, and D. L. Erdner (1996), Role of protozoan grazing in relieving iron limitation of phytoplankton, Nature, 380, 61-64.

Barbeau, K., G. Zhang, D. H. Live, and A. Butler (2002), Petrobactin, a photoreactive siderophore produced by the oil-degrading marine bacterium Marinobacter hydrocarbonoclasticus., J. Am. Chem. Soc., 124(3), 378-9.

Barbeau, K., E. L. Rue, C. G. Trick, K. W. Bruland, and A. Butler (2003), Photochemical reactivity of siderophores produced by marine heterotrophic bacteria and cyanobacteria, based on characteristic Fe(III) binding groups, Limnol. Oceanogr., 48(3), 1069-1078, doi:10.4319/1o.2003.48.3.1069.

Barbeau, K. A., E. L. Rue, K. W. Bruland, and A. Butler (2001), Photochemical cycling of iron in the surface ocean mediated by microbial iron(III)-binding ligands., Nature, 413(6854), 409-13, doi:10.1038/35096545.

Biller, D. V, and K. W. Bruland (2013), Sources and distributions of Mn , Fe , Co , Ni , Cu , Zn , and $\mathrm{Cd}$ relative to macronutrients along the central California coast during the spring and summer upwelling season, Mar. Chem., 155, 50-70, doi:10.1016/j.marchem.2013.06.003.

Biller, D. V, and K. W. Bruland (2014), The central California Current transition zone : A broad region exhibiting evidence for iron limitation, Prog. Oceanogr., 120, 370-382, doi:10.1016/j.pocean.2013.11.002. 
Biller, D. V., and K. W. Bruland (2012), Analysis of Mn, Fe, Co, Ni, Cu, Zn, Cd, and $\mathrm{Pb}$ in seawater using the Nobias-chelate PA1 resin and magnetic sector inductively coupled plasma mass spectrometry (ICP-MS), Mar. Chem., 130-131, 12-20, doi:10.1016/j.marchem.2011.12.001.

Biller, D. V., T. H. Coale, R. C. Till, G. J. Smith, and K. W. Bruland (2013), Coastal iron and nitrate distributions during the spring and summer upwelling season in the central California Current upwelling regime, Cont. Shelf Res., 66, 58-72, doi:10.1016/j.csr.2013.07.003.

Boiteau, R. M., and D. J. Repeta (2015), An extended siderophore suite from Synechococcus sp. PCC 7002 revealed by LC-ICPMS-ESIMS, Metallomics, 7(5), 877-884, doi:10.1039/C5MT00005J.

Boyle, E. A., J. M. Edmond, and E. R. Sholkovitz (1977), The mechanism of iron removal in estuaries, Geochim. Cosmochim. Acta, 41(9), 1313-1324, doi:10.1016/00167037(77)90075-8.

Bruland, K. W., R. P. Franks, G. a. Knauer, and J. H. Martin (1979), Sampling and analytical methods for the determination of copper, cadmium, zinc, and nickel at the nanogram per liter level in sea water, Anal. Chim. Acta, Vol. 105, 233-245, doi:10.1016/S00032670(01)83754-5.

Bruland, K. W., E. L. Rue, and G. J. Smith (2001), Iron and macronutrients in California coastal upwelling regimes: Implications for diatom blooms, Limnol. Oceanogr., 46(7), 1661-1674, doi:10.4319/1o.2001.46.7.1661.

Bruland, K. W., M. C. Lohan, A. M. Aguilar-Islas, G. J. Smith, B. Sohst, and A. Baptista (2008), Factors influencing the chemistry of the near-field Columbia River plume: Nitrate, silicic acid, dissolved $\mathrm{Fe}$, and dissolved $\mathrm{Mn}, \quad J$. Geophys. Res., 113, 1-23, doi:10.1029/2007JC004702.

Buck, K., M. Lohan, C. Berger, and K. Bruland (2007), Dissolved iron speciation in two distinct river plumes and an estuary: Implications for riverine iron supply, Limnol. Oceanogr., $52(2), 843-855$.

Bundy, R. M., D. V Biller, K. N. Buck, K. W. Bruland, and K. A. Barbeau (2014), Distinct pools of dissolved iron-binding ligands in the surface and benthic boundary layer of the California Current, Limnol. Oceanogr., 59(3), 769-787, doi:10.4319/lo.2014.59.3.0769.

Bundy, R. M., H. A. N. Abdulla, P. G. Hatcher, D. V. Biller, K. N. Buck, and K. a. Barbeau (2015a), Iron-binding ligands and humic substances in the San Francisco Bay estuary and estuarine-influenced shelf regions of coastal California, Mar. Chem., 173, 183-194, doi:10.1016/j.marchem.2014.11.005.

Bundy, R. M., H. A. N. Abdulla, P. G. Hatcher, D. V. Biller, K. N. Buck, and K. A. Barbeau 
(2015b), Iron-binding ligands and humic substances in the San Francisco Bay estuary and estuarine-influenced shelf regions of coastal California, Mar. Chem., 173, 183-194, doi:10.1016/j.marchem.2014.11.005.

Carpenter, L. J. (2003), Iodine in the marine boundary layer., Chem. Rev., 103(12), 4953-62, doi:10.1021/cr0206465.

Chase, Z., K. S. Johnson, V. A. Elrod, J. N. Plant, S. E. Fitzwater, L. Pickell, and C. M. Sakamoto (2005), Manganese and iron distributions off central California influenced by upwelling and shelf width, Mar. Chem., 95(3-4), 235-254, doi:10.1016/j.marchem.2004.09.006.

Checkley, D. M., and J. A. Barth (2009), Patterns and processes in the California Current System, Prog. Oceanogr., 83(1-4), 49-64, doi:10.1016/j.pocean.2009.07.028.

Chen, R. F., J. L. Bada, and Y. Suzuki (1993), The relationship between dissolved organic carbon (DOC) and fluorescence in anoxic marine porewaters: Implications for estimating benthic DOC fluxes, Geochim. Cosmochim. Acta, 57(9), 2149-2153, doi:10.1016/00167037(93)90102-3.

Cordero, O. X., L. A. Ventouras, E. F. DeLong, and M. F. Polz (2012), Public good dynamics drive evolution of iron acquisition strategies in natural bacterioplankton populations, Proc. Natl. Acad. Sci., 1-6, doi:10.1073/pnas.1213344109.

Cullen, J. T., B. A. Bergquist, and J. W. Moffett (2006), Thermodynamic characterization of the partitioning of iron between soluble and colloidal species in the Atlantic Ocean, Mar. Chem., 98(2-4), 295-303, doi:10.1016/j.marchem.2005.10.007.

D'Onofrio, A., J. M. Crawford, E. J. Stewart, K. Witt, E. Gavrish, S. Epstein, J. Clardy, and K. Lewis (2010), Siderophores from neighboring organisms promote the growth of uncultured bacteria., Chem. Biol., 17(3), 254-64, doi:10.1016/j.chembiol.2010.02.010.

Deicke, M., J. F. Mohr, J.-P. Bellenger, and T. Wichard (2014), Metallophore mapping in complex matrices by metal isotope coded profiling of organic ligands, Analyst, 139, 60966099, doi:10.1039/C4AN01461H.

Firme, G. F., E. L. Rue, D. A. Weeks, K. W. Bruland, and D. A. Hutchins (2003), Spatial and temporal variability in phytoplankton iron limitation along the California coast and consequences for Si, N, and C biogeochemistry, Global Biogeochem. Cycles, 17(1), 1016, doi:10.1029/2001gb001824.

Fitzsimmons, J., and E. Boyle (2012), An intercalibration between the GEOTRACES GO-FLO and the MITESS / Vanes sampling systems for dissolved iron concentration analyses (and a closer look at adsorption effects), Limnol. Oceanogr. Methods, 437-450, doi:10.4319/lom.2012.10.437. 
Fitzsimmons, J. N., R. M. Bundy, S. N. Al-Subiai, K. A. Barbeau, and E. A. Boyle (2014), The composition of dissolved iron in the dusty surface ocean: An exploration using sizefractionated iron-binding ligands, Mar. Chem., doi:10.1016/j.marchem.2014.09.002.

Gauglitz, J. M., and A. Butler (2013), Amino acid variability in the peptide composition of a suite of amphiphilic peptide siderophores from an open ocean Vibrio species., J. Biol. Inorg. Chem., 18(5), 489-97, doi:10.1007/s00775-013-0995-3.

Gledhill, M., P. McCormack, S. Ussher, E. P. Achterberg, R. F. C. Mantoura, and P. J. Worsfold (2004), Production of siderophore type chelates by mixed bacterioplankton populations in nutrient enriched seawater incubations, Mar. Chem., 88(1-2), 75-83, doi:10.1016/j.marchem.2004.03.003.

Grabo, J. E., M. A. Chrisman, L. M. Webb, and M. J. Baldwin (2014), Photochemical Reactivity of the Iron(III) Complex of a Mixed-Donor, $\alpha$-Hydroxy Acid-Containing Chelate and Its Biological Relevance to Photoactive Marine Siderophores, Inorg. Chem., (Iii), 140519080036009, doi:10.1021/ic500635q.

Hassler, C. S., V. Schoemann, C. M. Nichols, E. C. V. Butler, and P. W. Boyd (2011), Saccharides enhance iron bioavailability to Southern Ocean phytoplankton, Proc. Natl. Acad. Sci., 108(3), 1076-1081, doi:10.1073/pnas.1010963108.

Hassler, C. S., L. Norman, C. A. Mancuso Nichols, L. A. Clementson, C. Robinson, V. Schoemann, R. J. Watson, and M. A. Doblin (2014), Iron associated with exopolymeric substances is highly bioavailable to oceanic phytoplankton, Mar. Chem., doi:10.1016/j.marchem.2014.10.002.

Haygood, M. G., P. D. Holt, and A. Butler (1993), Aerobactin Production by a Planktonic Marine Vibrio sp., Limnol. Oceanogr., 38(5), 1091-1097.

Hertkorn, N., M. Harir, K. M. Cawley, P. Schmitt-Kopplin, and R. Jaffé (2015), Molecular characterization of dissolved organic matter from subtropical wetlands: a comparative study through the analysis of optical properties, NMR and FTICR/MS, Biogeosciences Discuss., 12(16), 13711-13765, doi:10.5194/bgd-12-13711-2015.

Hibbing, M. E., C. Fuqua, M. R. Parsek, and S. B. Peterson (2010), Bacterial competition: surviving and thriving in the microbial jungle., Nat. Rev. Microbiol., 8(1), 15-25, doi:10.1038/nrmicro2259.

Homann, V. V, M. Sandy, J. A. Tincu, A. S. Templeton, B. M. Tebo, and A. Butler (2009), Loihichelins A-F, a suite of amphiphilic siderophores produced by the marine bacterium Halomonas LOB-5., J. Nat. Prod., 72(5), 884-8, doi:10.1021/np800640h.

Hutchins, D. A., and K. W. Bruland (1998), Iron-limited diatom growth and Si:N uptake ratios in a coastal upwelling regime, Nature, 393(June), 65-68. 
Hutchins, D. A., A. E. Witter, A. Butler, and G. W. Luther (1999a), Competition among marine phytoplankton for different chelated iron species, Nature, 400(6747), 858-861.

Hutchins, D. A., V. M. Franck, M. A. Brzezinski, and K. W. Bruland (1999b), Inducing phytoplankton iron limitation in iron-replete coastal waters with a strong chelating ligand, Limnol. Oceanogr., 44(4), 1009-1018, doi:10.4319/1o.1999.44.4.1009.

Ito, Y., and A. Butler (2005), Structure of synechobactins, new siderophores of the marine cyanobacterium Synechococcus sp. PCC 7002, Limnol. Oceanogr., 50(6), 1918-1923, doi:10.4319/1o.2005.50.6.1918.

Jalal, M., M. Hossain, D. Van der Helm, J. Sanders-Loehr, L. Actis, and J. Crosa (1989), Structure of anguibactin, a unique plasmid-related bacterial siderophore from the fish pathogen Vibrio anguillarum, J. Am. Chem. Soc., 111(1), 292-296.

John, S. G., J. Mendez, J. Moffett, and J. Adkins (2012), The flux of iron and iron isotopes from San Pedro Basin sediments, Geochim. Cosmochim. Acta, 93, 14-29, doi:10.1016/j.gca.2012.06.003.

Keith Johnson, W., L. A. Miller, N. E. Sutherland, and C. S. Wong (2005), Iron transport by mesoscale Haida eddies in the Gulf of Alaska, Deep Sea Res. Part II Top. Stud. Oceanogr., 52(7-8), 933-953, doi:10.1016/j.dsr2.2004.08.017.

King, A. L., and K. Barbeau (2007), Evidence for phytoplankton iron limitation in the southern California Current System, Mar. Ecol. Prog. Ser., 342, 91-103, doi:10.3354/meps342091.

King, A. L., S. A. Sañudo-Wilhelmy, P. W. Boyd, B. S. Twining, S. W. Wilhelm, C. Breene, M. J. Ellwood, and D. A. Hutchins (2012a), A comparison of biogenic iron quotas during a diatom spring bloom using multiple approaches, Biogeosciences, 9(2), 667-687, doi:10.5194/bg-9-667-2012.

King, A. L., K. N. Buck, and K. A. Barbeau (2012b), Quasi-Lagrangian drifter studies of iron speciation and cycling off Point Conception, California, Mar. Chem., 128-129, 1-12, doi:10.1016/j.marchem.2011.11.001.

Kirchman, D. L., B. Meon, M. T. Cottrell, D. A. Hutchins, D. Weeks, and K. W. Bruland (2000), Carbon versus iron limitation of bacterial growth in the California upwelling regime, Limnology, 45(8), 1681-1688.

Laglera, L. M., and C. M. G. van den Berg (2009), Evidence for geochemical control of iron by humic substances in seawater, Limnol. Oceanogr., 54(2), 610-619.

Laglera, L. M., G. Battaglia, and C. M. G. van den Berg (2011), Effect of humic substances on the iron speciation in natural waters by CLE/CSV, Mar. Chem., 127(1-4), 134-143, doi:10.1016/j.marchem.2011.09.003. 
Lohan, M. C., and K. W. Bruland (2008), Elevated Fe(II) and dissolved Fe in hypoxic shelf waters off Oregon and Washington: An enhanced source of iron to coastal upwelling regimes, Environ. Sci. Technol., 42(17), 6462-6468, doi:10.1021/es800144j.

Macrellis, H. M., C. G. Trick, E. L. Rue, G. Smith, and K. W. Bruland (2001), Collection and detection of natural iron-binding ligands from seawater, Mar. Chem., 76(3), 175-187, doi:10.1016/S0304-4203(01)00061-5.

Martin, J. D., Y. Ito, V. V Homann, M. G. Haygood, and A. Butler (2006), Structure and membrane affinity of new amphiphilic siderophores produced by Ochrobactrum sp. SP18., J. Biol. Inorg. Chem., 11(5), 633-41, doi:10.1007/s00775-006-0112-y.

Martinez, J., G. Zhang, P. Holt, and H. Jung (2000), Self-assembling amphiphilic siderophores from marine bacteria, Science, 287(5456), 1245-1247, doi:10.1126/science.287.5456.1245.

Martinez, J. S., and A. Butler (2007), Marine amphiphilic siderophores: marinobactin structure, uptake, and microbial partitioning, J. Inorg. Biochem., 101(805), 1692-1698, doi:10.1016/j.jinorgbio.2007.07.007.Marine.

Martinez, J. S., M. G. Haygood, and A. Butler (2001), Identification of a natural desferrioxamine siderophore produced by a marine bacterium, Limnol. Oceanogr., 46(2), 420-424, doi:10.4319/1o.2001.46.2.0420.

Mawji, E., M. Gledhill, J. A. Milton, G. A. Tarran, S. Ussher, A. Thompson, G. A. Wolff, P. J. Worsfold, and E. P. Achterberg (2008), Hydroxamate siderophores: occurrence and importance in the Atlantic Ocean., Environ. Sci. Technol., 42(23), 8675-80.

Mawji, E., M. Gledhill, J. A. Milton, M. V. Zubkov, A. Thompson, G. A. Wolff, and E. P. Achterberg (2011), Production of siderophore type chelates in Atlantic Ocean waters enriched with different carbon and nitrogen sources, Mar. Chem., 124(1-4), 90-99, doi:10.1016/j.marchem.2010.12.005.

McCormack, P., P. J. Worsfold, and M. Gledhill (2003), Separation and detection of siderophores produced by marine bacterioplankton using high-performance liquid chromatography with electrospray ionization mass spectrometry., Anal. Chem., 75(11), $2647-52$.

Méndez-Díaz, J. D., K. K. Shimabuku, J. Ma, Z. O. Enumah, J. J. Pignatello, W. a Mitch, and M. C. Dodd (2014), Sunlight-driven photochemical halogenation of dissolved organic matter in seawater: a natural abiotic source of organobromine and organoiodine., Environ. Sci. Technol., 48(13), 7418-27, doi:10.1021/es5016668.

Moore, R. M., M. Webb, R. Tokarczyk, and R. Wever (1996), Bromoperoxidase and iodoperoxidase enzymes and production of halogenated methanes in marine diatom cultures, J. Geophys. Res., 101(C9), 20899, doi:10.1029/96JC01248. 
Mucha, P., P. Rekowski, A. Kosakowska, and G. Kupryszewski (1999), Separation of siderophores by capillary electrophoresis, J. Chromatogr. A, 830(1), 183-189, doi:10.1016/S0021-9673(98)00907-8.

Parsons, T. R., Y. Maita, C. M. Lalli, and . A manual of chemical and biological methods for seawater Analysis." (1984), A manual of chemical and biological methods for seawater analysis, Pergamon Press Ltd., Oxford, England.

Peterson, T. D., F. a. Whitney, and P. J. Harrison (2005), Macronutrient dynamics in an anticyclonic mesoscale eddy in the Gulf of Alaska, Deep. Res. Part II Top. Stud. Oceanogr., 52, 909-932, doi:10.1016/j.dsr2.2005.02.004.

Peterson, T. D., D. W. Crawford, and P. J. Harrison (2011), Mixing and biological production at eddy margins in the eastern Gulf of Alaska, Deep. Res. Part I Oceanogr. Res. Pap., 58(4), 377-389, doi:10.1016/j.dsr.2011.01.010.

Repeta, D. J. (2015), Chemical characterization and cycling of dissolved organic matter, in Biogeochemistry of Marine Dissolved Organic Matter, edited by D. A. Hansell and C. A. Carlson, Elsevier.

Rose, A. L., and T. D. Waite (2003), Kinetics of hydrolysis and precipitation of ferric iron in seawater., Environ. Sci. Technol., 37(17), 3897-903.

Rykaczewski, R. R., and D. M. Checkley (2008), Influence of ocean winds on the pelagic ecosystem in upwelling regions., Proc. Natl. Acad. Sci., 105(6), 1965-1970, doi:10.1073/pnas.0711777105.

Sandy, M., A. Han, J. Blunt, M. Munro, M. Haygood, and A. Butler (2010), Vanchrobactin and anguibactin siderophores produced by Vibrio sp. DS40M4., J. Nat. Prod., 73(6), 1038-43, doi:10.1021/np900750g.

Sato, M., S. Takeda, and K. Furuya (2007), Iron regeneration and organic iron(III)-binding ligand production during in situ zooplankton grazing experiment, Mar. Chem., 106, 471488, doi:10.1016/j.marchem.2007.05.001.

Shaked, Y., A. B. Kustka, and F. M. M. Morel (2005), A general kinetic model for iron acquisition by eukaryotic phytoplankton, Limnol. Oceanogr., 50(3), 872-882.

Völker, C., and D. A. Wolf-Gladrow (1999), Physical limits on iron uptake mediated by siderophores or surface reductases, Mar. Chem., 65(3-4), 227-244, doi:10.1016/S03044203(99)00004-3.

Vraspir, J. M., and A. Butler (2009), Chemistry of Marine Ligands and Siderophores, Ann. Rev. Mar. Sci., 1(1), 43-63, doi:10.1146/annurev.marine.010908.163712.

Vraspir, J. M., P. D. Holt, and A. Butler (2011), Identification of new members within suites of 
amphiphilic marine siderophores., Biometals, 24(1), 85-92, doi:10.1007/s10534-010-93781.

Waska, H., A. Koschinsky, M. J. Ruiz Chancho, and T. Dittmar (2015), Investigating the potential of solid-phase extraction and Fourier-transform ion cyclotron resonance mass spectrometry (FT-ICR-MS) for the isolation and identification of dissolved metal-organic complexes from natural waters, Mar. Chem., 173, 78-92, doi:10.1016/j.marchem.2014.10.001.

Wheatcroft, R. A., C. K. Sommerfield, D. E. Drake, J. C. Borgeld, and C. A. Nittrouer (1997), Rapid and widespread dispersal of flood sediment on the northern California margin, Geology, 25(2), 163-166, doi:10.1130/0091-7613(1997)025<0163:RAWDOF>2.3.CO;2.

Xu, J. P., M. Noble, and S. L. Eittreim (2002), Suspended sediment transport on the continental shelf near Davenport, California, Mar. Geol., 181(1-3), 171-193, doi:10.1016/S00253227(01)00266-3.

Zane, H. K., H. Naka, F. Rosconi, M. Sandy, M. G. Haygood, and A. Butler (2014), Biosynthesis of Amphi-enterobactin Siderophores by Vibrio harveyi BAA-1116: Identification of a Bifunctional Nonribosomal Peptide Synthetase Condensation Domain, J. Am. Chem. Soc., 136(15), 5615-5618, doi:10.1021/ja5019942. 


\subsection{Figures:}

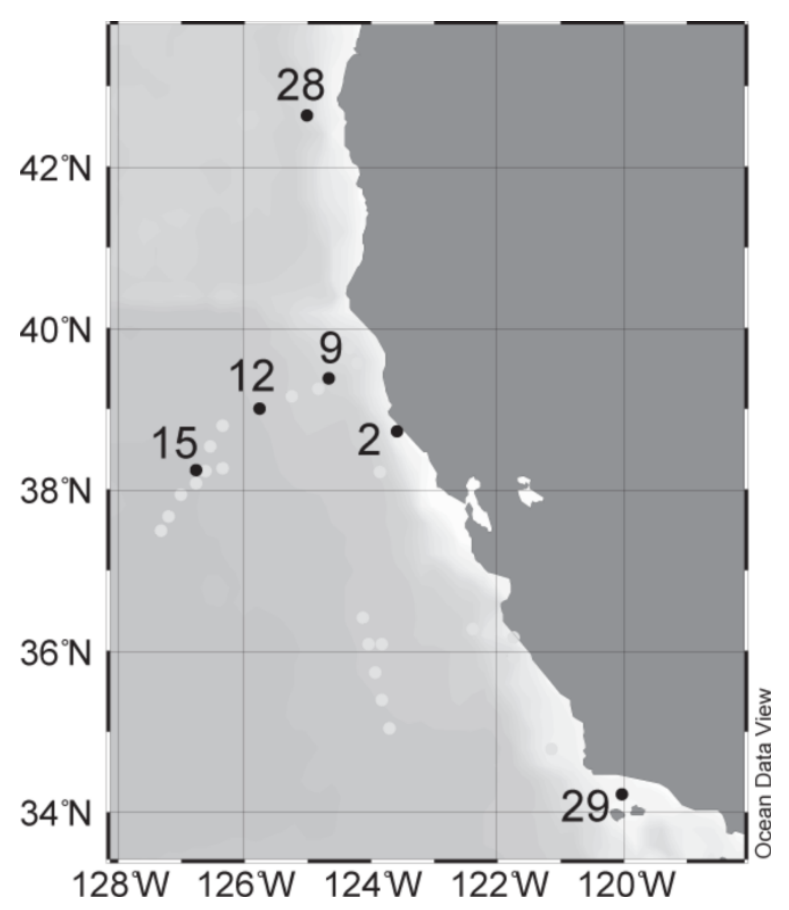

Figure 5.1: Map of California coast and station sampling locations during the IRNBRU cruise. 
A

\section{Station 2}
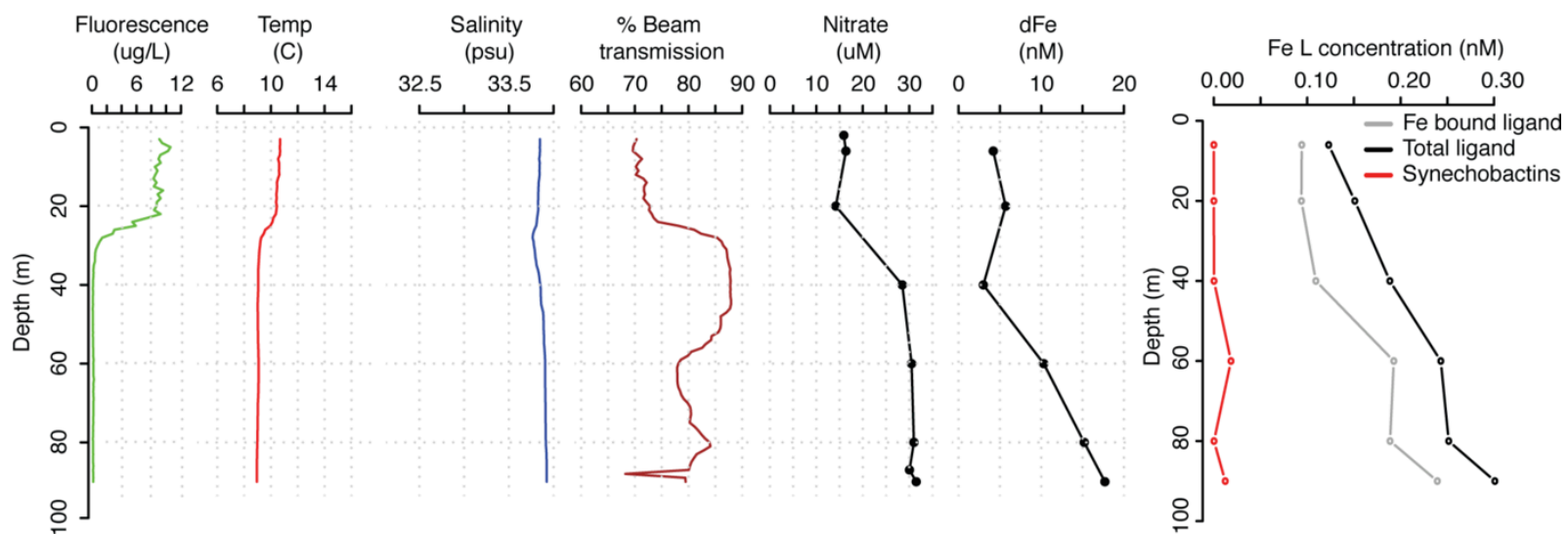

B

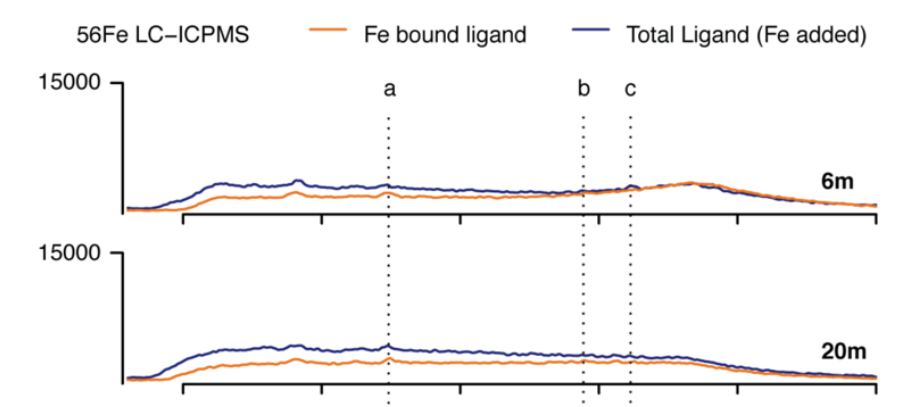

C

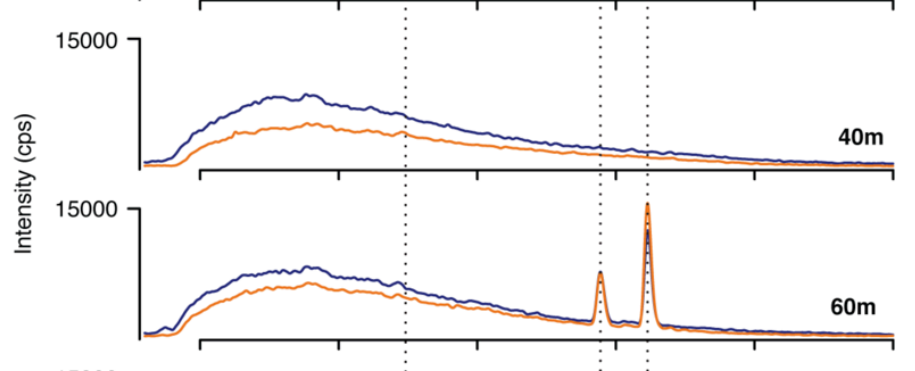

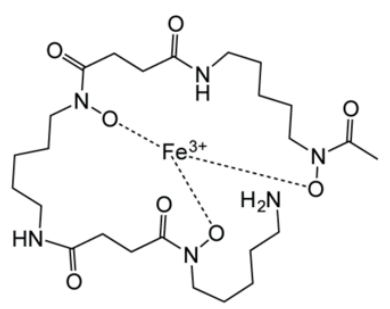

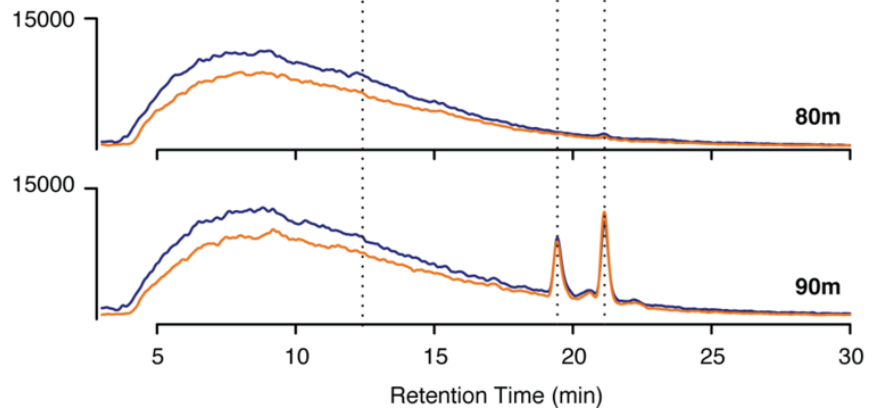

b
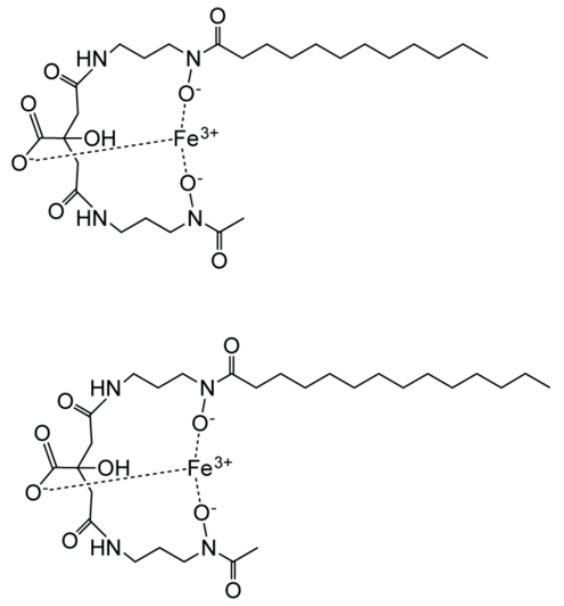

Figure 5.2: Station 2, Wide shelf upwelling region. (a) Depth profiles of CTD parameters, nitrate, dissolved iron, and LC-ICPMS based FeL concentrations. (b) ${ }^{56} \mathrm{Fe}$ LC-ICPMS chromatograms of organic extracts collected from each depth. Dashed lines indicate chromatographic peaks from compounds that were identified by LC-ESIMS (c) structure of identified ligands a - ferrioxamine B, $\mathrm{b}$ - synechobactin C12, $\mathrm{c}$ - synechobactin C14. 
A

Station 28
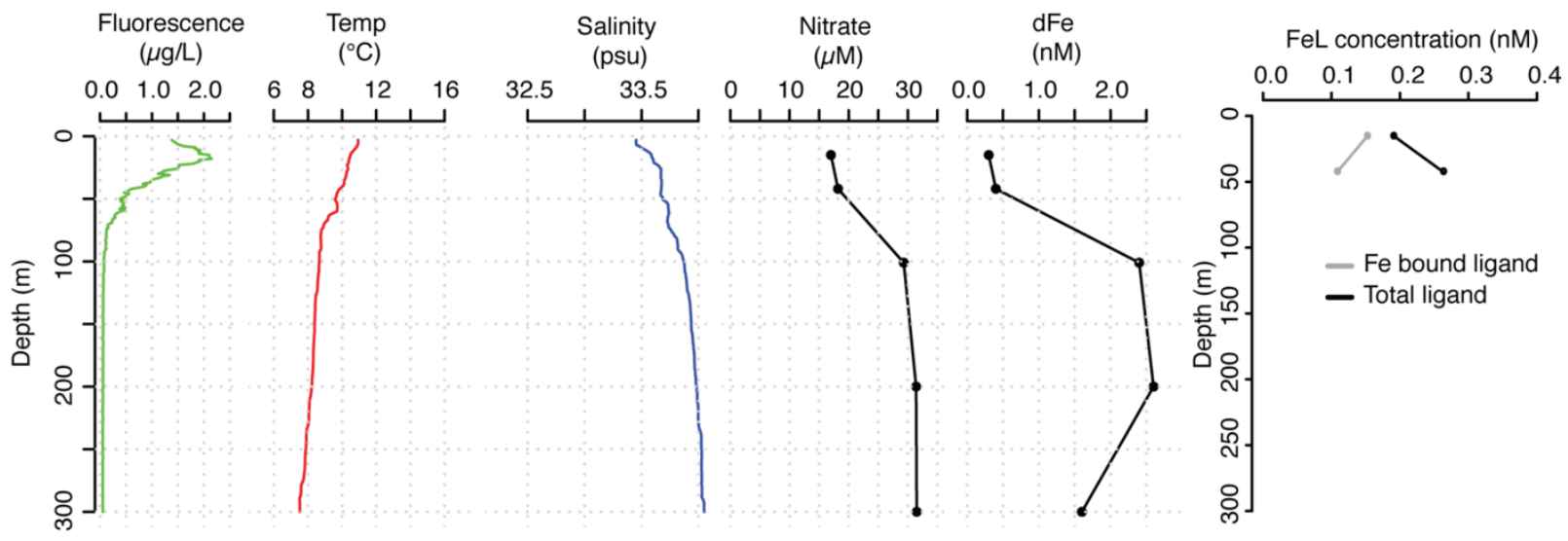

B

56Fe LC-ICPMS - Fe bound ligand $\quad-$ Total ligand (Fe added)

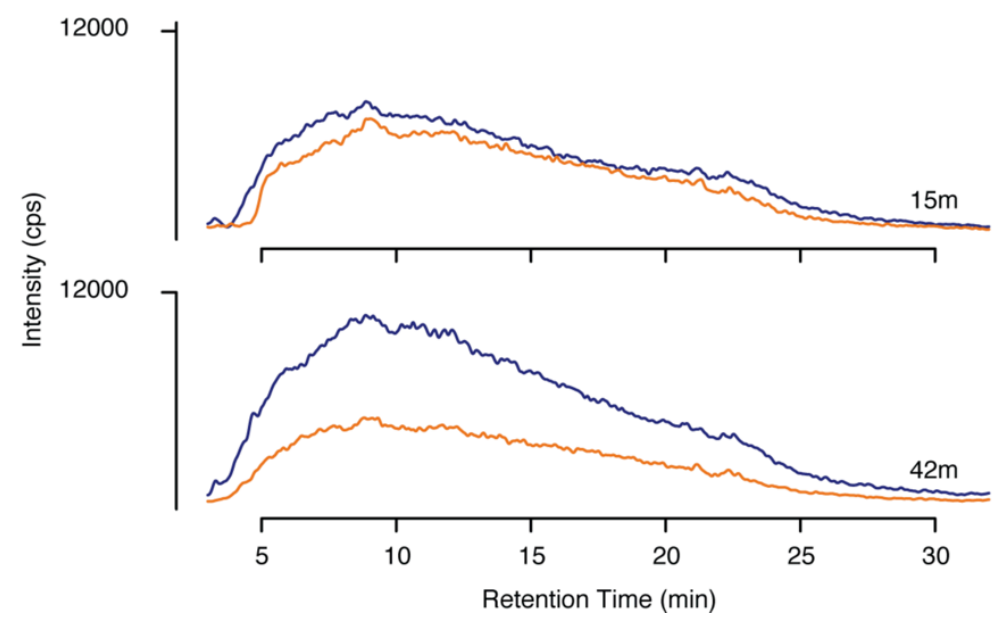

Figure 5.3: Station 28, Narrow shelf upwelling region (a) Depth profiles of CTD parameters, nitrate, dissolved iron, and LC-ICPMS based FeL concentrations. (b) ${ }^{56} \mathrm{Fe}$ LC-ICPMS chromatograms of organic extracts collected from each depth. 
A

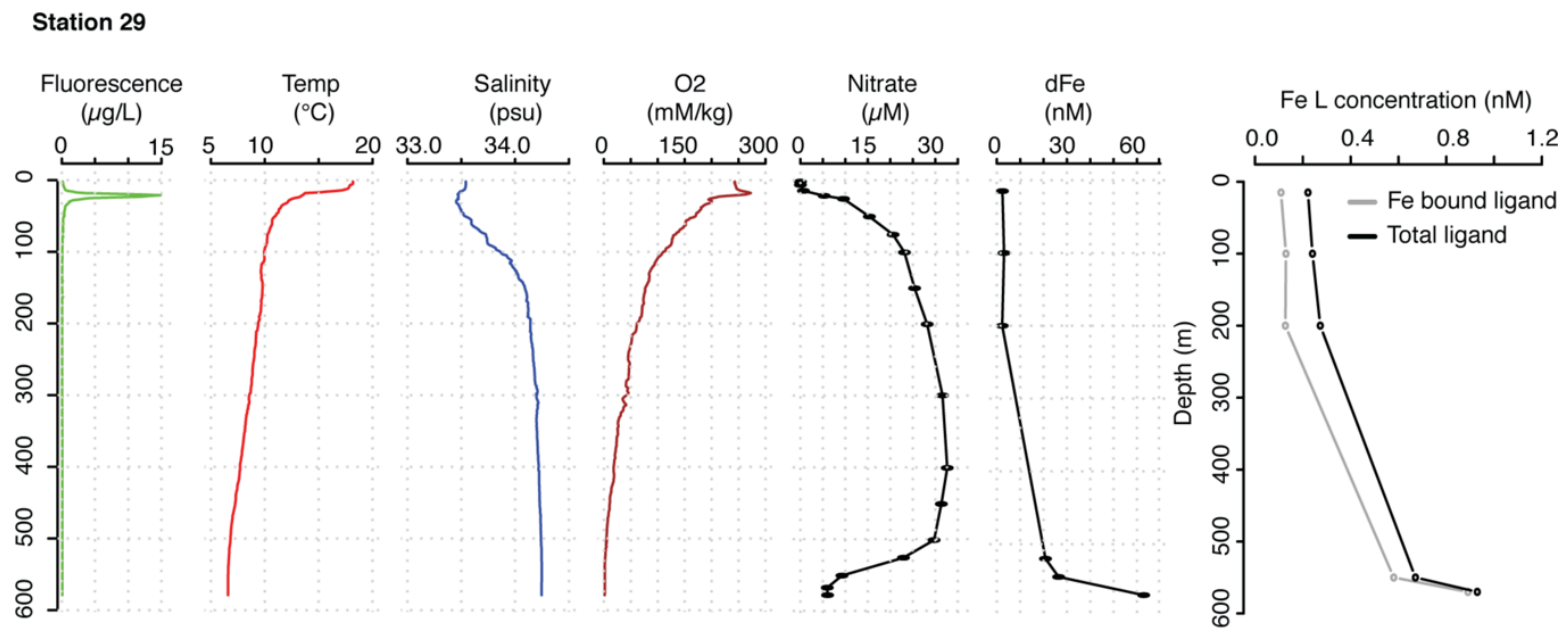

B

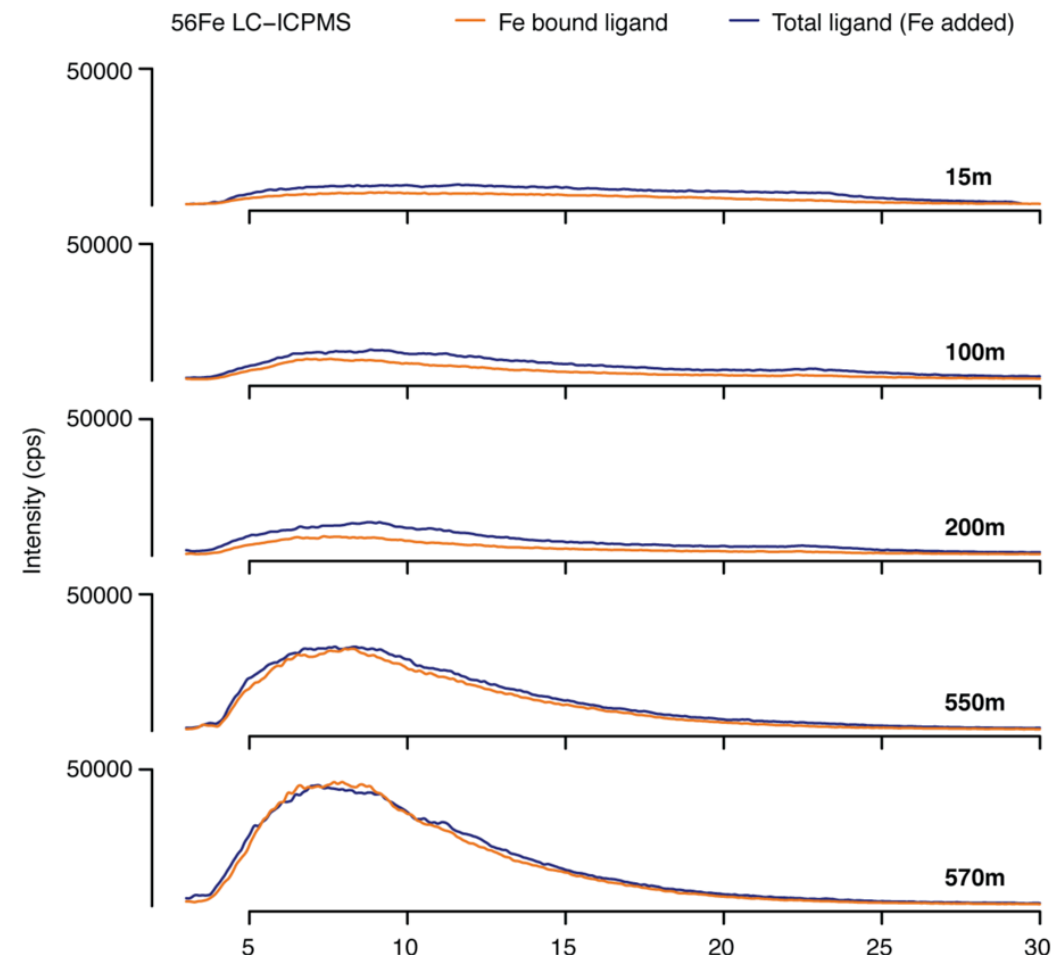

Figure 5.4: Station 29 - Santa Barbara basin station (a) Depth profiles of CTD parameters, nitrate, dissolved iron, and LC-ICPMS based FeL concentrations. (b) ${ }^{56} \mathrm{Fe}$ LC-ICPMS chromatograms of organic extracts collected from each depth. 
a

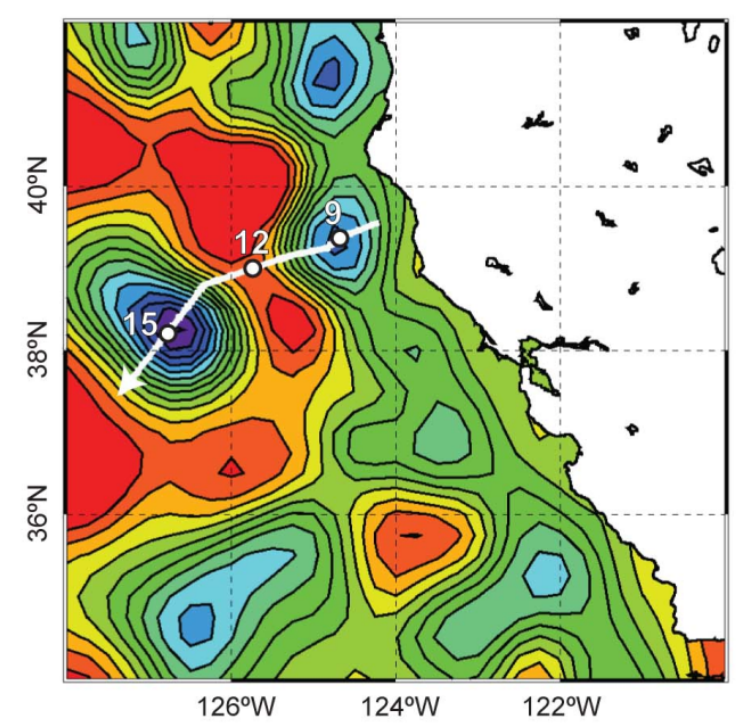

b
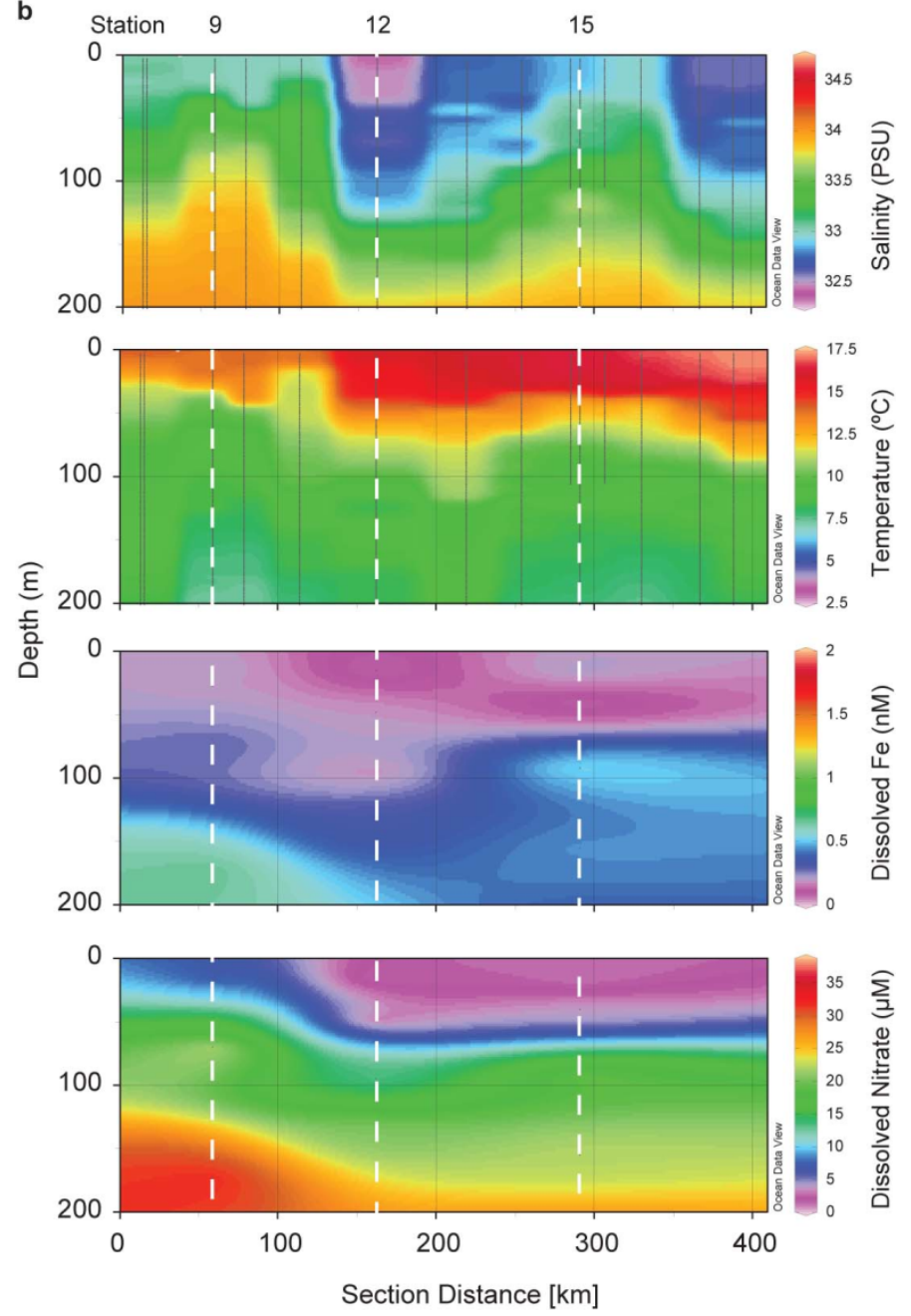

Figure 5.5: (a) Map of transect across CCS eddies, showing sea surface height anomaly $(+14 \mathrm{~cm}=\mathrm{red},-14 \mathrm{~cm}=$ blue $)$. (b) Depth profiles of salinity, temperature, dissolved $\mathrm{Fe}$, and dissolved N. Stations where samples for LC-ICPMS were collected are shown as dashed lines. 
Station 9 (nearshore cyclonic eddie)
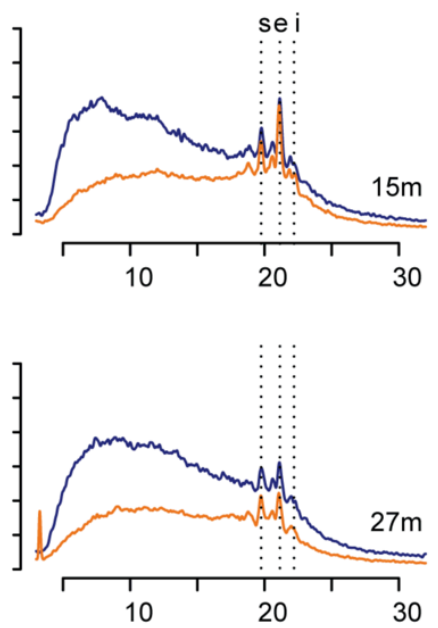

Retention Time (min)

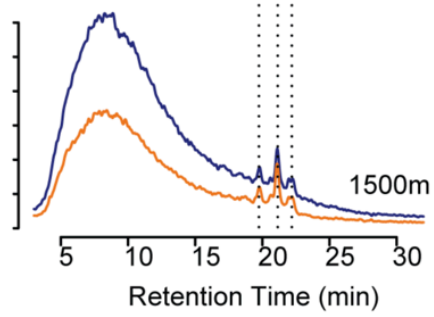

Station 12 (anticyclonic eddie)
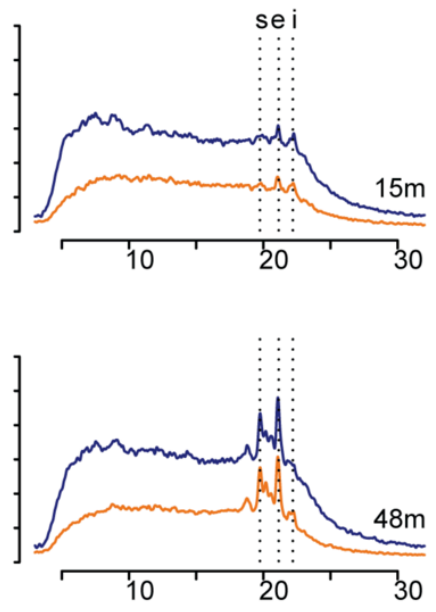

- No Fe added (FeL)

- Fe added (Ltotal)

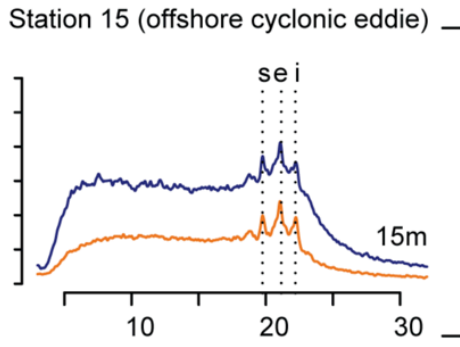

Surface

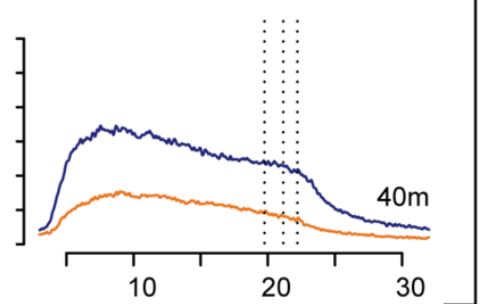

Deep

Chlorophll

Max

Mesopelagic

b
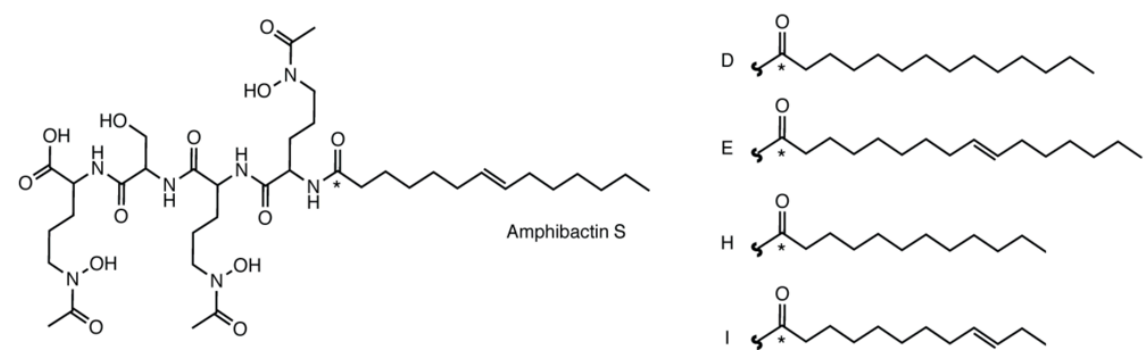

Figure 5.6: (a) LC-ICPMS chromatograms of ${ }^{56} \mathrm{Fe}$ from samples collected across three eddies within the transition zone between the upwelling coast and the California current. Labeled peaks correspond to amphibactins s, e and i. (b) Structure of amphibactins detected across the CCS. 
a

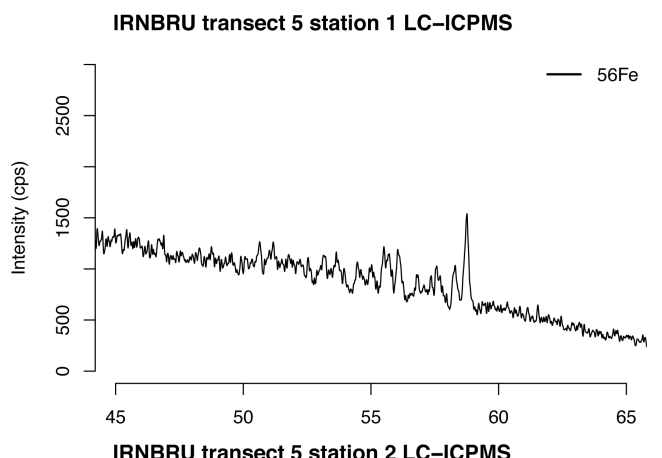

IRNBRU transect 5 station 2 LC-ICPMS
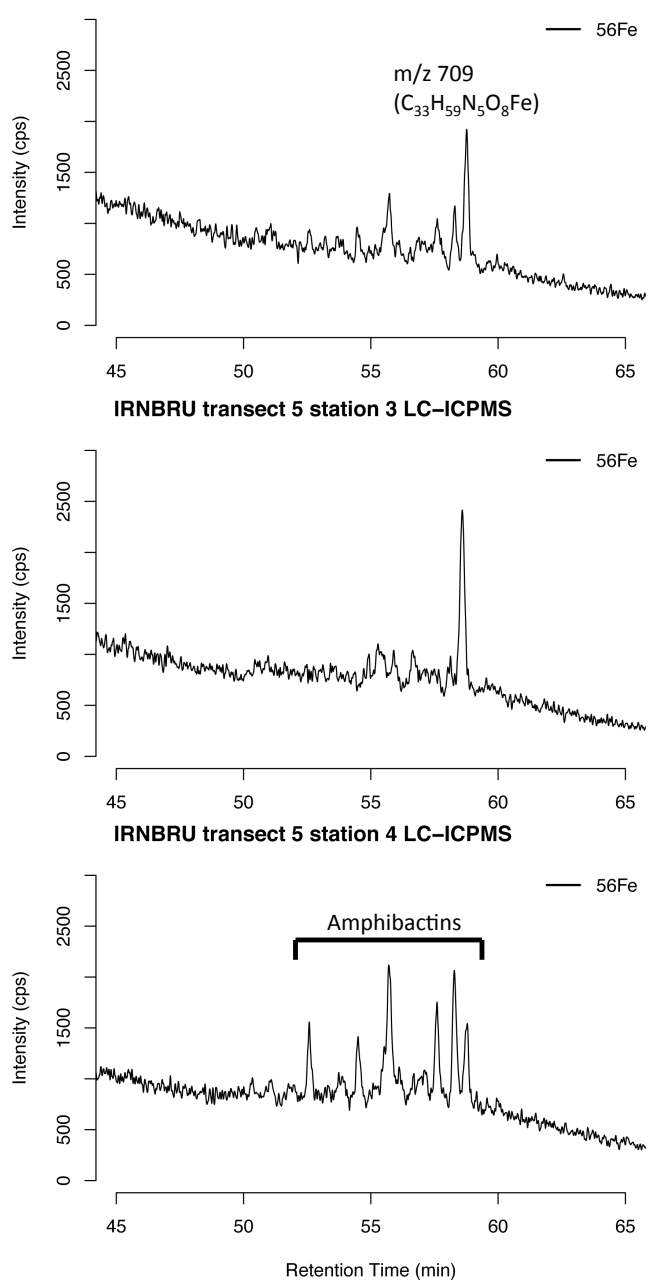

b
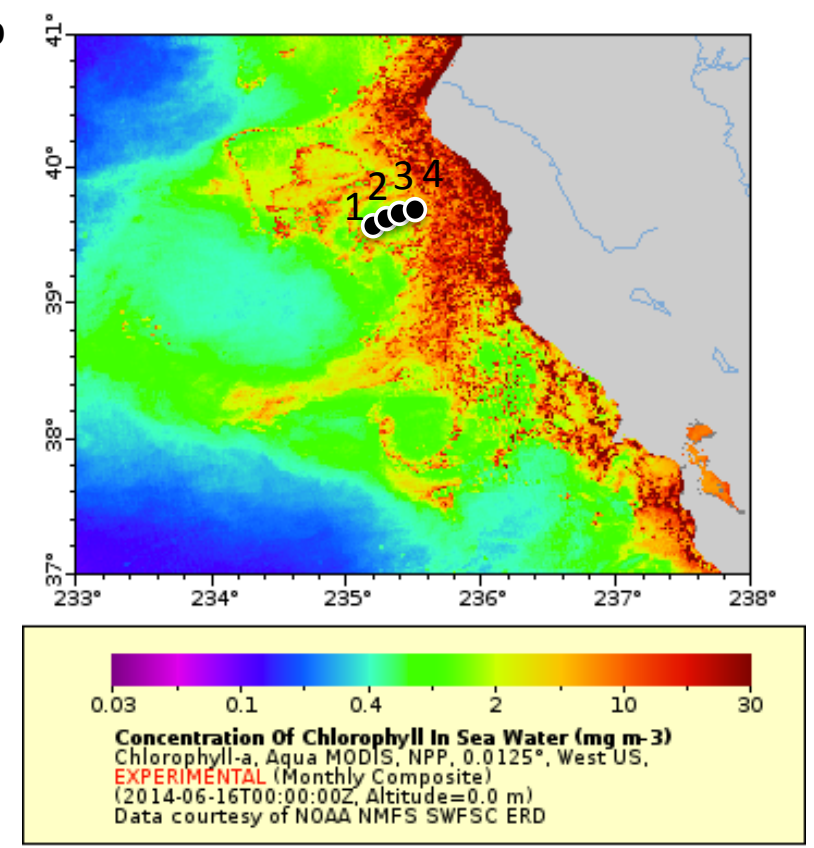

Figure 5.7: (a) Changes in LC-ICPMS chromatograms of iron ligands across a cyclonic eddy (near station 9) (b) Satellite derived surface ocean concentrations of chlorophyll B (average for month of June 2014). 
a

(⿸丆口广

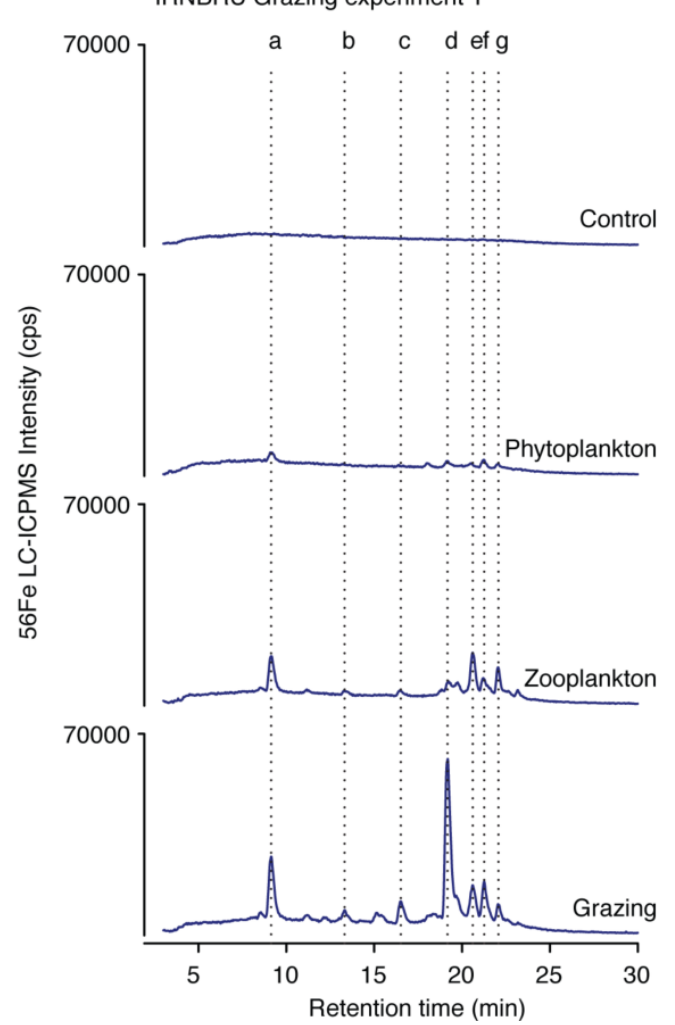

b

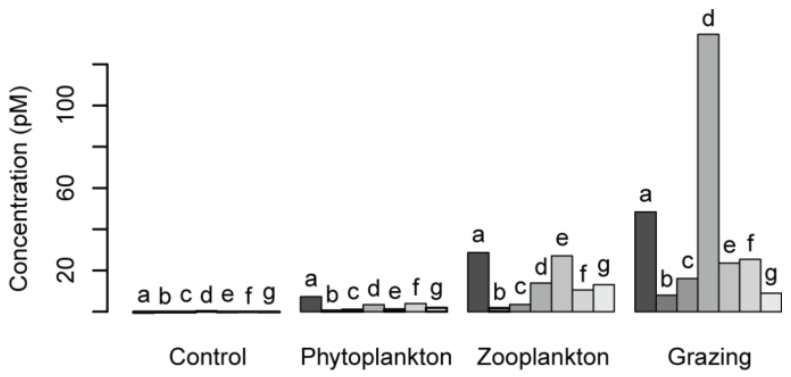

Figure 5.8: (a) LC-ICPMS chromatograms of iron ligands detected during zooplankton grazing experiment. Letters indicate major peaks that correspond to compounds in Table 2. (b) Concentration of ligand from grazing experiment. 


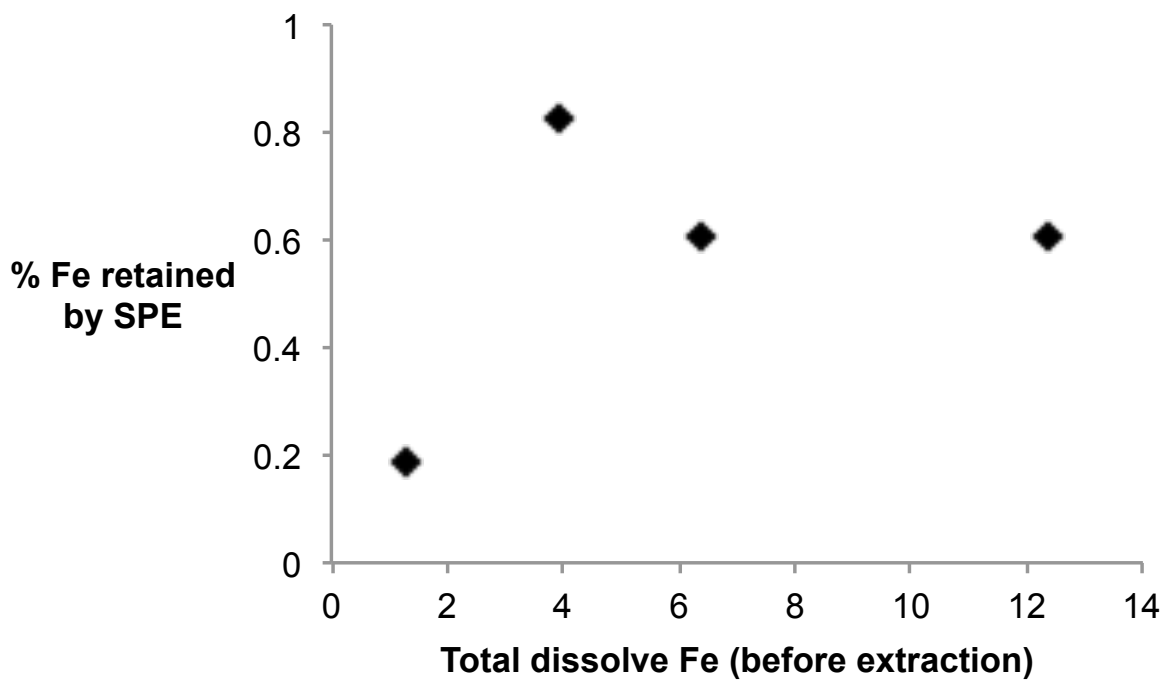

Figure 5.9: SPE extraction efficiency for iron. Dissolved iron concentrations were measured before and after the seawater was passed through an ENV solid phase extraction column. 

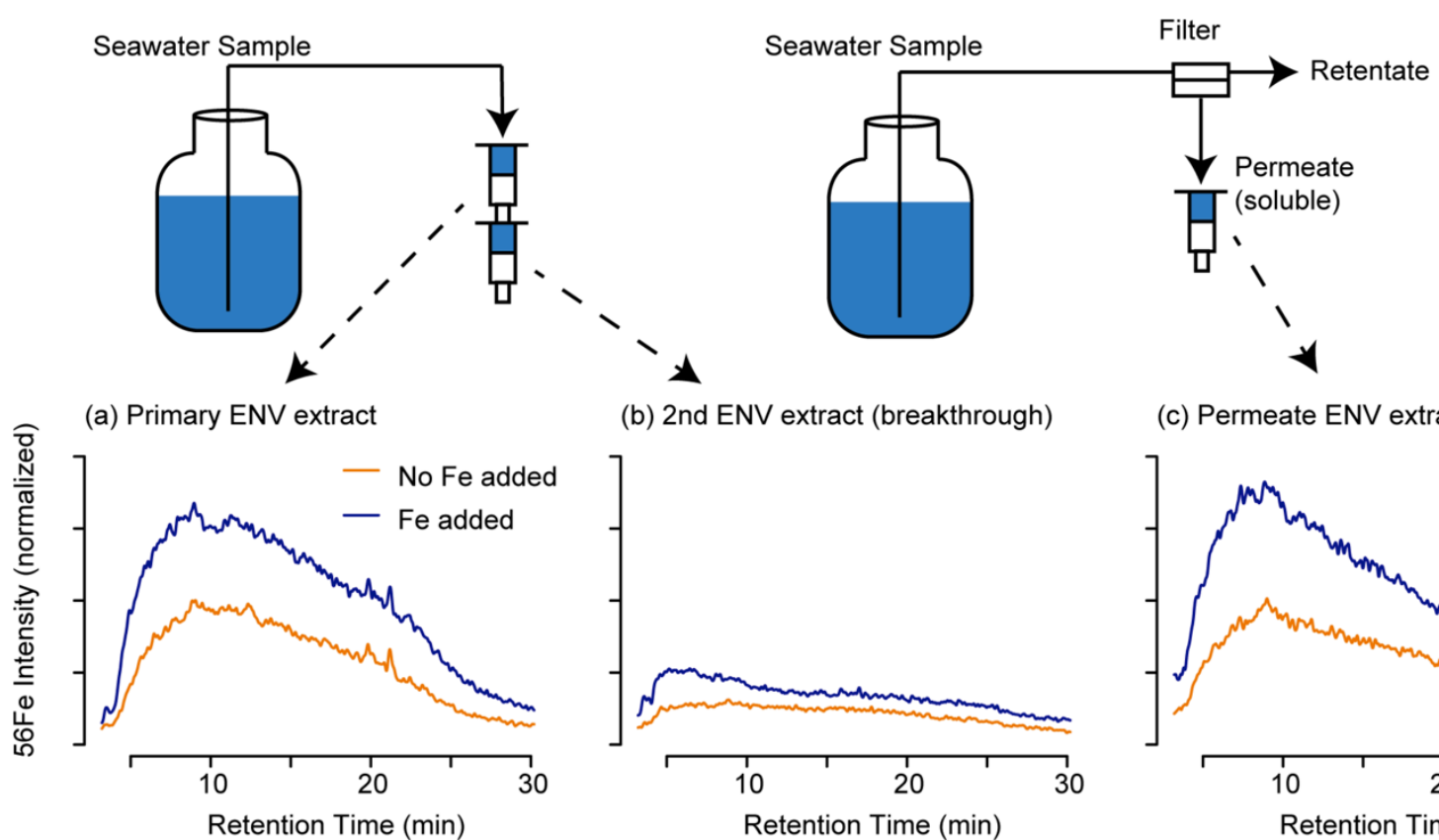

(b) 2nd ENV extract (breakthrough)

(c) Permeate ENV extract (soluble)

Figure 5.10: Comparison of primary, breakthrough, and soluble seawater ENV extract. Two 20L volumes of seawater collected simultaneously were extracted through either two ENV SPE columns, or were passed through a tangential flow filter with a $1 \mathrm{kd}$ cutoff, and only the permeate was extracted. The intensities of the chromatograms have been scaled relative to the volume extracted (10L). 
a
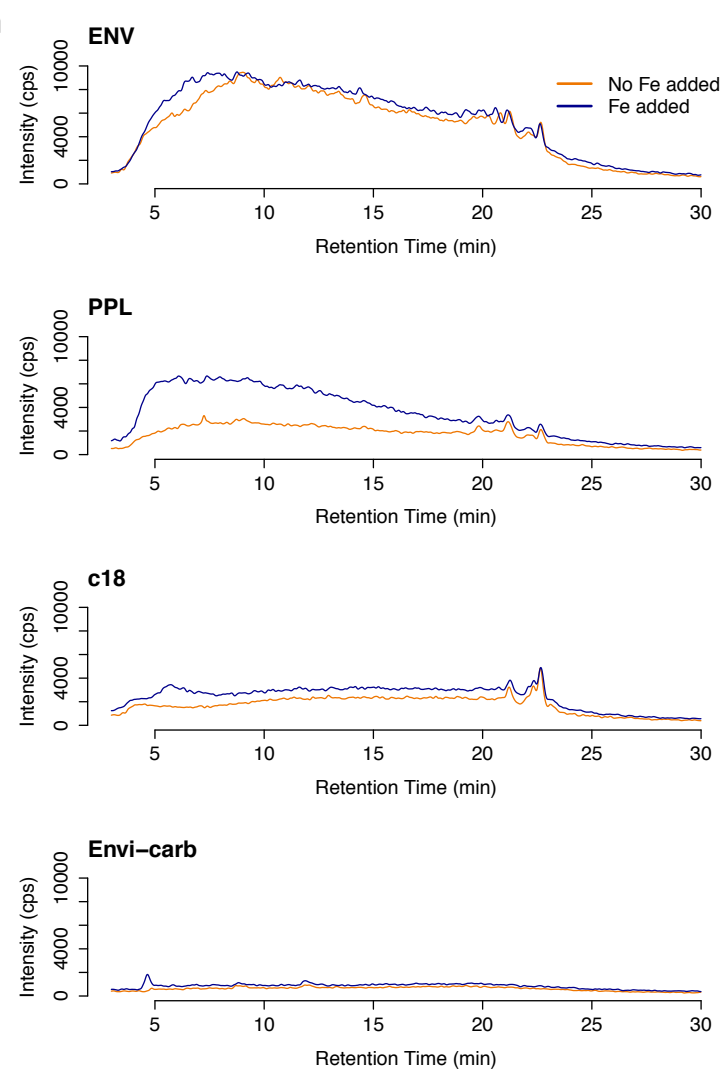
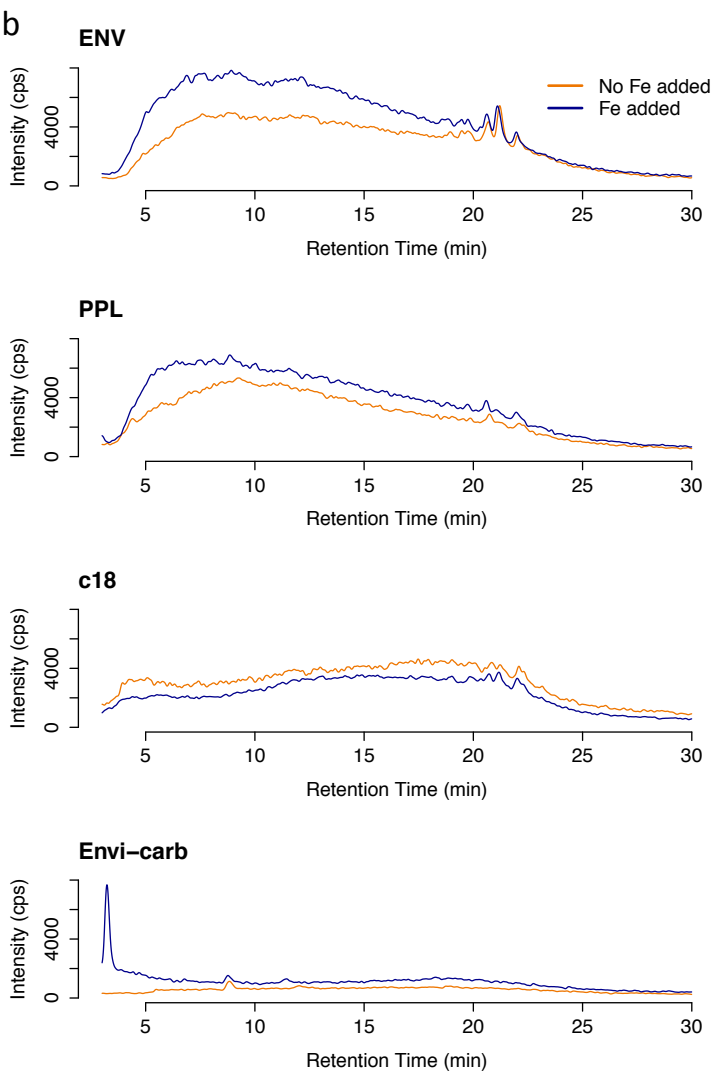

Figure 5.11: LC-ICPMS chromatograms of seawater organic extracts from stations 6 (a) and 20 (b) collected with four different solid phase extraction resins. ENV and PPL are polystyrene based resins. C18 is a functionalized silica based resin. Envi-carb is an activated charcoal based resin. 
Table 5.1: Identification of iron binding compounds in CCS seawater

\begin{tabular}{lll}
\hline Elution Time $(\mathrm{min})$ & ${ }^{56} \mathrm{Fe}$ monoisotopic & \\
{$[\mathrm{M}+\mathrm{H}]^{+} \mathrm{m} / \mathrm{z}$} & Identity \\
\hline 19.4 & 614.261 & Synechobactin A \\
21.1 & 642.292 & Synechobactin $\mathrm{C} 14$ \\
22.9 & 709.371 & $\mathrm{C}_{33} \mathrm{H}_{59} \mathrm{~N}_{5} \mathrm{O}_{8} \mathrm{Fe}^{+*}$ \\
19.7 & 883.399 & Amphibactin S \\
20.5 & 885.415 & Amphibactin D \\
21.1 & 911.43 & Amphibactin $\mathrm{E}$ \\
21.9 & 913.446 & Amphibactin $\mathrm{H}$ \\
22.2 & 939.462 & Amphibactin I \\
*Hypothesized chemical formula &
\end{tabular}


Table 2: Identification of Fe compounds in the IRNBRU grazing experiment

\begin{tabular}{llll}
\hline Peak & Elution time $(\mathrm{min})$ & $\begin{array}{l}{ }^{56} \mathrm{Fe} \text { monoisotopic } \\
{[\mathrm{M}+\mathrm{H}]^{+} \mathrm{m} / \mathrm{z}}\end{array}$ \\
\hline \multirow{3}{*}{$\mathrm{a}$} & 8.7 & 700.273 & unknown \\
& 9.1 & 672.278 & Ferrioxamine G \\
$\mathrm{d}$ & 11.1 & 654.267 & Ferrioxamine $\mathrm{E}$ \\
& 19 & 968.342 & Unknown \\
$\mathrm{e}$ & 19.7 & 876.430 & Unknown \\
& 20.5 & 902.446 & Unknown \\
$\mathrm{f}$ & 20.65 & 854.445 & Unknown \\
$\mathrm{g}$ & 21.3 & 884.456 & Unknown \\
& 22.06 & 882.477 & Unknown
\end{tabular}


Table 3: Description of CCS samples for ligand characterization collected on the IRNBRU cruise

\begin{tabular}{|c|c|c|c|c|c|c|c|}
\hline Sample ID & SPE Resin* & Date collected & Station & Latitude & Longitude & Depth & Region ${ }^{\#}$ \\
\hline 1 & ENV & $7 / 6 / 14$ & 2 & 38.72 & 123.58 & $6 m$ & Upwelling \\
\hline 2 & ENV & $7 / 6 / 14$ & 2 & 38.72 & 123.58 & $20 m$ & Upwelling \\
\hline 3 & ENV & $7 / 6 / 14$ & 2 & 38.72 & 123.58 & $40 m$ & Upwelling \\
\hline 4 & ENV & $7 / 6 / 14$ & 2 & 38.72 & 123.58 & $60 m$ & Upwelling \\
\hline 5 & ENV & $7 / 6 / 14$ & 2 & 38.72 & 123.58 & $80 m$ & Upwelling \\
\hline 6 & ENV & $7 / 6 / 14$ & 2 & 38.72 & 123.58 & $90 m$ & Upwelling \\
\hline 7 & ENV & $7 / 9 / 14$ & 6 & 38.22 & 123.83 & surface & Transition \\
\hline 8 & PPL & $7 / 9 / 14$ & 6 & 38.22 & 123.83 & surface & Transition \\
\hline 9 & Envi-carb & $7 / 9 / 14$ & 6 & 38.22 & 123.83 & surface & Transition \\
\hline 10 & C18 & $7 / 9 / 14$ & 6 & 38.22 & 123.83 & surface & Transition \\
\hline 11 & ENV & $7 / 11 / 14$ & 9 & 39.38 & 124.67 & $15 \mathrm{~m}$ & Transition \\
\hline 12 & ENV & $7 / 11 / 14$ & 9 & 39.38 & 124.67 & $27 m$ & Transition \\
\hline 13 & ENV & $7 / 11 / 14$ & 12 & 39.00 & 125.75 & $15 \mathrm{~m}$ & Transition \\
\hline 14 & ENV & $7 / 11 / 14$ & 12 & 39.00 & 125.75 & $48 m$ & Transition \\
\hline 15 & ENV & $7 / 12 / 14$ & 15 & 38.25 & 126.75 & $15 \mathrm{~m}$ & Transition \\
\hline 16 & ENV & $7 / 12 / 14$ & 15 & 38.25 & 126.75 & $40 m$ & Transition \\
\hline 17 & ENV & $7 / 13 / 14$ & 12 & 39.00 & 125.75 & $1500 \mathrm{~m}$ & Deep \\
\hline 18 & ENV & $7 / 13 / 14$ & Transect 5 & 39.28 & 124.81 & surface & Transition \\
\hline 19 & ENV & $7 / 13 / 14$ & Transect 5 & 39.35 & 124.71 & surface & Transition \\
\hline 20 & ENV & $7 / 14 / 14$ & Transect 5 & 39.38 & 124.66 & surface & Transition \\
\hline 21 & ENV & $7 / 14 / 14$ & Transect 5 & 39.43 & 124.54 & surface & Transition \\
\hline 22 & ENV & $7 / 16 / 14$ & 21 & 35.93 & 121.73 & $65 \mathrm{~m}$ & Transition \\
\hline 23 & ENV & $7 / 16 / 14$ & 21 & 35.93 & 121.73 & $81 \mathrm{~m}$ & Transition \\
\hline 24 & ENV & $7 / 17 / 14$ & 22 & 36.28 & 122.38 & surface & Transition \\
\hline 25 & 2nd ENV & $7 / 17 / 14$ & 22 & 36.28 & 122.38 & surface & Transition \\
\hline 26 & PPL & $7 / 17 / 14$ & 22 & 36.28 & 122.38 & surface & Transition \\
\hline 27 & Envi-carb & $7 / 17 / 14$ & 22 & 36.28 & 122.38 & surface & Transition \\
\hline 28 & C18 & $7 / 17 / 14$ & 22 & 36.28 & 122.38 & surface & Transition \\
\hline 29 & ENV & $7 / 18 / 14$ & 25 & 35.41 & 123.92 & $30 \mathrm{~m}$ & Transition \\
\hline 30 & ENV & $7 / 18 / 14$ & 25 & 35.41 & 123.92 & $118 \mathrm{~m}$ & Transition \\
\hline 31 & ENV & $7 / 20 / 14$ & Transect 9 & 42.71 & -125.06 & surface & Transition \\
\hline 32 & ENV & $7 / 20 / 14$ & Transect 9 & 42.87 & -125.29 & surface & Transition \\
\hline 33 & ENV & $7 / 20 / 14$ & Transect 9 & 42.74 & -125.46 & surface & Transition \\
\hline 34 & ENV & $7 / 20 / 14$ & Transect 9 & 42.63 & -125.61 & surface & Transition \\
\hline 35 & ENV & $7 / 20 / 14$ & Transect 9 & 42.51 & -125.76 & surface & Transition \\
\hline 36 & ENV & $7 / 20 / 14$ & Transect 9 & 42.38 & -125.92 & surface & Transition \\
\hline 37 & ENV & $7 / 21 / 14$ & 28 & 42.66 & 125.00 & $15 \mathrm{~m}$ & Upwelling \\
\hline 38 & ENV & $7 / 21 / 14$ & 28 & 42.66 & 125.00 & $42 m$ & Upwelling \\
\hline 39 & ENV & $7 / 22 / 14$ & None & 38.89 & 123.96 & surface & Transition \\
\hline 40 & 2nd ENV & $7 / 22 / 14$ & None & 38.89 & 123.96 & surface & Transition \\
\hline 41 & ENV (after TFF) & $7 / 22 / 14$ & None & 38.89 & 123.96 & surface & Transition \\
\hline
\end{tabular}




\begin{tabular}{rlllrlll}
\hline Sample ID & SPE Resin & Date collected & Station & Latitude & Longitude & Depth & Region \\
\hline 42 & ENV & $7 / 24 / 14$ & 29 & 34.30 & 120.06 & $15 \mathrm{~m}$ & SBB- Oxic $^{\#}$ \\
43 & ENV & $7 / 24 / 14$ & 29 & 34.30 & 120.06 & $100 \mathrm{~m}$ & SBB- Oxic \\
44 & ENV & $7 / 24 / 14$ & 29 & 34.30 & 120.06 & $200 \mathrm{~m}$ & SBB- Oxic \\
45 & ENV & $7 / 24 / 14$ & 29 & 34.30 & 120.06 & $550 \mathrm{~m}$ & SBB-Anoxic \\
46 & ENV & $7 / 24 / 14$ & 29 & 34.30 & 120.06 & $570 \mathrm{~m}$ & SBB-Anoxic
\end{tabular}

*For two samples, four different solid phase extraction resins were tested: ENV (Agilent, 1g, 6mL column), PPL (Agilent, 1g, 6mL column), c18 (Agilent, 1g, 6mL column), and Envi-carb (Supleco, $0.5 \mathrm{~g}, 6 \mathrm{~mL}$ column). Grey values indicate replicate samples that were collected from the same locations using different extraction methods.

${ }^{\#}$ Region corresponds to sampling location. Upwelling regions were characterized by cold surface waters and high surface iron concentrations $<12$ degrees $C$, Transition zone was characterized by warm surface waters ( $>12$ degrees C). Santa Barbara Basin (SBB) samples were characterized by oxic or anoxic conditions. 
Table 4: Nutrient and iron concentrations, iron ligand concentrations (Fe bound and total, determined by LC-ICPMS), and siderophores detected from CCS samples.

\begin{tabular}{|c|c|c|c|c|c|c|}
\hline $\begin{array}{l}\text { Sample } \\
\text { ID }\end{array}$ & $\begin{array}{l}\text { Nitrate } \\
\text { (uM) }\end{array}$ & $\begin{array}{l}\text { Silicate } \\
\text { (uM) }\end{array}$ & $\begin{array}{l}\mathrm{dFe} \\
(\mathrm{nmol} / \mathrm{kg})\end{array}$ & $\begin{array}{l}\text { FeL conc } \\
(\mathrm{nM})\end{array}$ & $\begin{array}{l}\text { Ltotal conc } \\
(\mathrm{nM})\end{array}$ & Siderophores detected: \\
\hline 1 & 16.37 & 28.54 & 2.84 & 0.094 & 0.123 & Ferrioxamine B \\
\hline 2 & 14.20 & 25.35 & 3.76 & 0.094 & 0.151 & Ferrioxamine B \\
\hline 3 & 28.50 & 31.26 & 4.38 & 0.109 & 0.188 & None \\
\hline 4 & 30.50 & 41.31 & 12.16 & 0.193 & 0.243 & Synechobactins \\
\hline 5 & 31.00 & 41.14 & 14.81 & 0.189 & 0.251 & None \\
\hline 6 & 31.50 & 42.36 & 16.53 & 0.239 & 0.301 & Synechobactins \\
\hline 7 & 8.26 & 3.36 & $\mathrm{~N} / \mathrm{A}$ & 0.105 & 0.160 & Amphibactin D, E, H, I \\
\hline 8 & 8.26 & 3.36 & $\mathrm{~N} / \mathrm{A}$ & 0.089 & 0.127 & \\
\hline 9 & 8.26 & 3.36 & $\mathrm{~N} / \mathrm{A}$ & 0.031 & 0.031 & \\
\hline 10 & 8.26 & 3.36 & N/A & 0.006 & 0.026 & \\
\hline 11 & 5.16 & 2.94 & 0.22 & 0.087 & 0.165 & Amphibactin S, D, E, H, I \\
\hline 12 & 10.02 & 8.11 & 0.16 & 0.077 & 0.177 & Amphibactin S, D, E, H, I \\
\hline 13 & 0.08 & 1.50 & 0.03 & 0.064 & 0.163 & Amphibactins E, $\mathrm{H}, \mathrm{I}$ \\
\hline 14 & 0.54 & 1.84 & 0.19 & 0.080 & 0.197 & $\begin{array}{l}\text { Amphibactin S, D, E, H, I, } \\
\text { unknown } 709\end{array}$ \\
\hline 15 & 0.41 & 1.89 & 0.25 & 0.064 & 0.177 & Amphibactin S, D, E, H, I \\
\hline 16 & 3.90 & 2.98 & 0.03 & 0.054 & 0.173 & No siderophores \\
\hline 17 & 43.94 & 140.15 & 1.07 & 0.091 & 0.185 & Amphibactins S, D , E, H, I \\
\hline 18 & 4.23 & 2.40 & $\mathrm{~N} / \mathrm{A}$ & $\mathrm{N} / \mathrm{A}$ & $\mathrm{N} / \mathrm{A}$ & unknown 709.371 \\
\hline 19 & 3.88 & 2.23 & 0.14 & $\mathrm{~N} / \mathrm{A}$ & $\mathrm{N} / \mathrm{A}$ & unknown 709.371 \\
\hline 20 & 4.85 & 2.99 & 0.08 & $\mathrm{~N} / \mathrm{A}$ & $\mathrm{N} / \mathrm{A}$ & unknown 709.371 \\
\hline 21 & 5.08 & 3.26 & 0.12 & $\mathrm{~N} / \mathrm{A}$ & $\mathrm{N} / \mathrm{A}$ & $\begin{array}{l}\text { Amphibactin S, D, E, H, I, } \\
\text { unknown } 709\end{array}$ \\
\hline 22 & 17.41 & 18.43 & $\mathrm{~N} / \mathrm{A}$ & 0.182 & 0.206 & Amphibactins S, D,E , H, I \\
\hline 23 & 21.08 & 24.19 & $\mathrm{~N} / \mathrm{A}$ & 0.152 & 0.243 & Amphibactins S, D,E , H, I \\
\hline 24 & $\mathrm{~N} / \mathrm{A}$ & $\mathrm{N} / \mathrm{A}$ & $\mathrm{N} / \mathrm{A}$ & 0.175 & 0.192 & $\begin{array}{l}\text { Amphibactin S, D, E, H, I, } \\
\text { unknown } 709\end{array}$ \\
\hline 25 & $\mathrm{~N} / \mathrm{A}$ & $\mathrm{N} / \mathrm{A}$ & $\mathrm{N} / \mathrm{A}$ & 0.047 & 0.067 & \\
\hline 26 & $\mathrm{~N} / \mathrm{A}$ & $\mathrm{N} / \mathrm{A}$ & N/A & 0.077 & 0.156 & \\
\hline 27 & $\mathrm{~N} / \mathrm{A}$ & $\mathrm{N} / \mathrm{A}$ & $\mathrm{N} / \mathrm{A}$ & 0.028 & 0.039 & \\
\hline 28 & N/A & $\mathrm{N} / \mathrm{A}$ & $\mathrm{N} / \mathrm{A}$ & 0.062 & 0.085 & \\
\hline 29 & 0.22 & 2.08 & 0.2 & 0.173 & 0.190 & Amphibactins S, D,E , H, I \\
\hline 30 & 18.02 & 14.49 & 2.0 & 0.173 & 0.311 & Amphibactins S, D,E , H, I \\
\hline 31 & 19.97 & 22.74 & 0.36 & 0.095 & 0.201 & $\begin{array}{l}\text { Amphibactin D, E, H, I, } \\
\text { unknown } 709\end{array}$ \\
\hline 32 & 2.58 & 5.19 & 0.34 & 0.093 & 0.304 & $\begin{array}{l}\text { Amphibactin D, E, H, I, } \\
\text { unknown } 709\end{array}$ \\
\hline 33 & 15.50 & 16.73 & 0.31 & 0.078 & 0.214 & $\begin{array}{l}\text { Amphibactin D, E, H, I, } \\
\text { unknown } 709\end{array}$ \\
\hline 34 & 10.32 & 6.78 & 0.26 & 0.145 & 0.169 & $\begin{array}{l}\text { Amphibactin D, E, H, I, } \\
\text { unknown } 709\end{array}$ \\
\hline 35 & 6.64 & 1.79 & 0.30 & 0.131 & 0.184 & $\begin{array}{l}\text { Amphibactin D, E, H, I, } \\
\text { unknown } 709\end{array}$ \\
\hline
\end{tabular}




\begin{tabular}{lllllll}
\hline $\begin{array}{l}\text { Sample } \\
\text { ID }\end{array}$ & $\begin{array}{l}\text { Nitrate } \\
(\mathrm{uM})\end{array}$ & $\begin{array}{l}\text { Silicate } \\
(\mathrm{uM})\end{array}$ & $\begin{array}{l}\mathrm{dFe} \\
(\mathrm{nmol} / \mathrm{kg})\end{array}$ & $\begin{array}{l}\text { FeL conc } \\
(\mathrm{nM})\end{array}$ & $\begin{array}{l}\text { Ltotal conc } \\
(\mathrm{nM})\end{array}$ & Siderophores detected: \\
\hline & 4.41 & 5.10 & 0.31 & 0.119 & 0.181 & $\begin{array}{l}\text { Amphibactin D, E, H, I, } \\
\text { unknown 709 }\end{array}$ \\
36 & & & & & & None \\
37 & 17 & 18.8 & 0.72 & 0.153 & 0.191 & None \\
38 & 18.2 & 19.96 & 0.53 & 0.109 & 0.264 & Amphibactin S, D, E, H, I \\
39 & $\mathrm{~N} / \mathrm{A}$ & $\mathrm{N} / \mathrm{A}$ & $\mathrm{N} / \mathrm{A}$ & 0.065 & 0.131 & \\
40 & $\mathrm{~N} / \mathrm{A}$ & $\mathrm{N} / \mathrm{A}$ & $\mathrm{N} / \mathrm{A}$ & 0.010 & 0.029 & \\
41 & $\mathrm{~N} / \mathrm{A}$ & $\mathrm{N} / \mathrm{A}$ & $\mathrm{N} / \mathrm{A}$ & 0.038 & 0.107 & None \\
42 & 0.02 & 0.2 & 4.58 & 0.109 & 0.221 & None \\
43 & 25.1 & 29 & 7.91 & 0.129 & 0.240 & None \\
44 & 29.5 & 88.18 & 11.43 & 0.127 & 0.273 & None \\
45 & 16.2 & 104.7 & 30.38 & 0.580 & 0.671 & None \\
46 & 10.8 & 115.9 & 56.92 & 0.891 & 0.930 &
\end{tabular}




\section{Appendix 5.1: Siderophore identification}

56Fe LC-ICPMS

Synechobactin A' (c12)

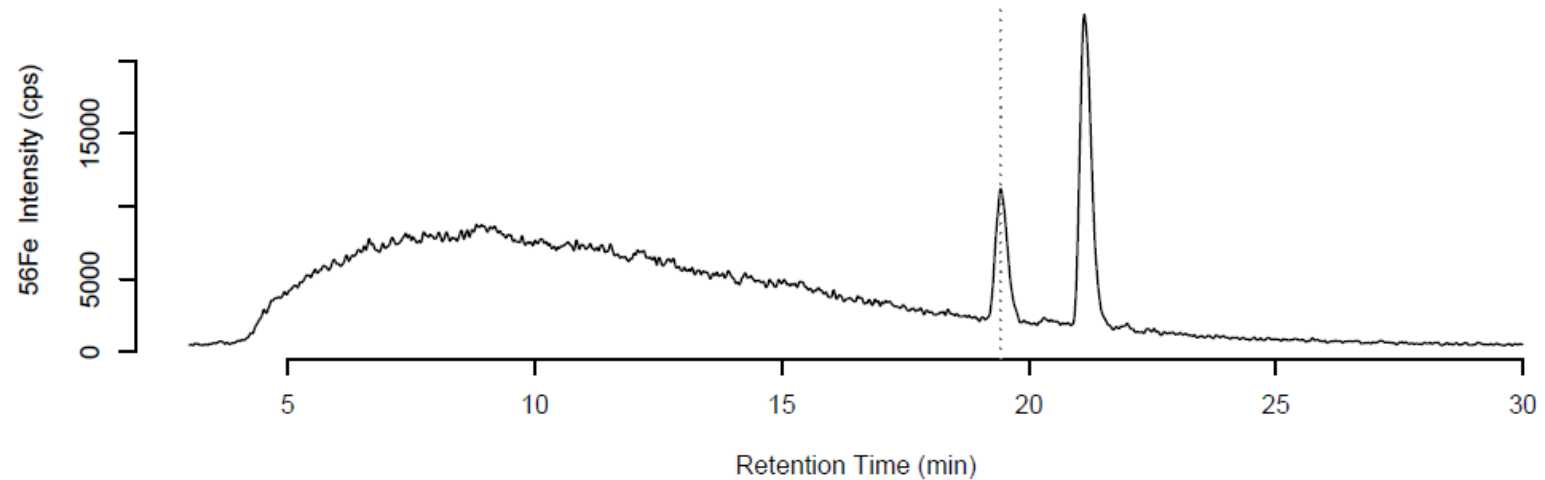

Isotope peaks LC-ESIMS. EIC $=612.266,614.262$

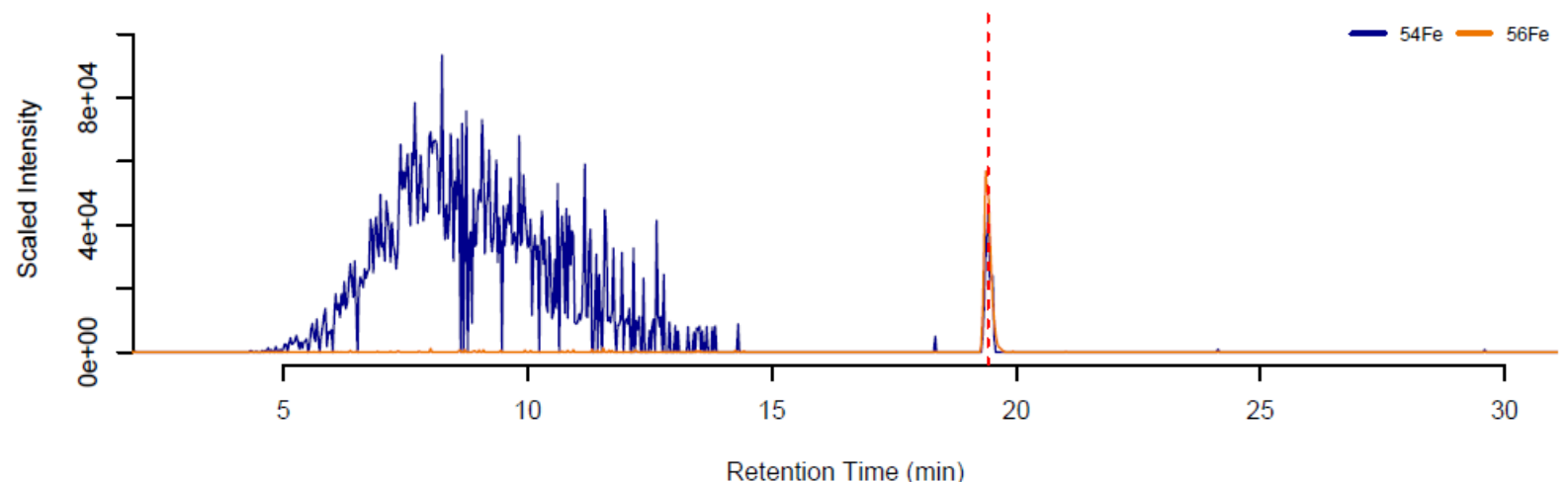

MS2 fragmentation. Precursor $=614.26$ scan time $=19.35 \mathrm{~min}$

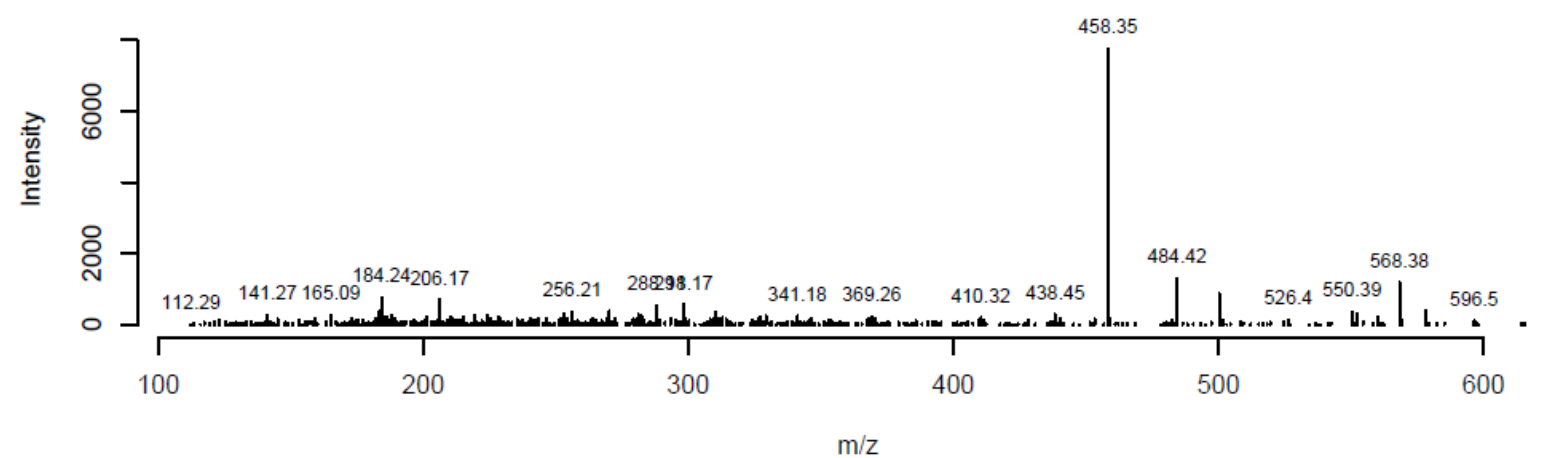

Figure A5.1-1: (top panel) 56Fe LC-ICPMS chromatogram (middle panel) LC-ESIMS extracted ion chromatograms for the $54 \mathrm{Fe}$ (blue) and $56 \mathrm{Fe}$ (orange) containing isotopologues $[\mathrm{M}+\mathrm{H}]^{+}$ ions. (bottom panel) MS2 fragmentation pattern for the $56 \mathrm{Fe}[\mathrm{M}+\mathrm{H}]^{+}$ion. Data collected from $60 \mathrm{~m}$ sample, Station 2. 


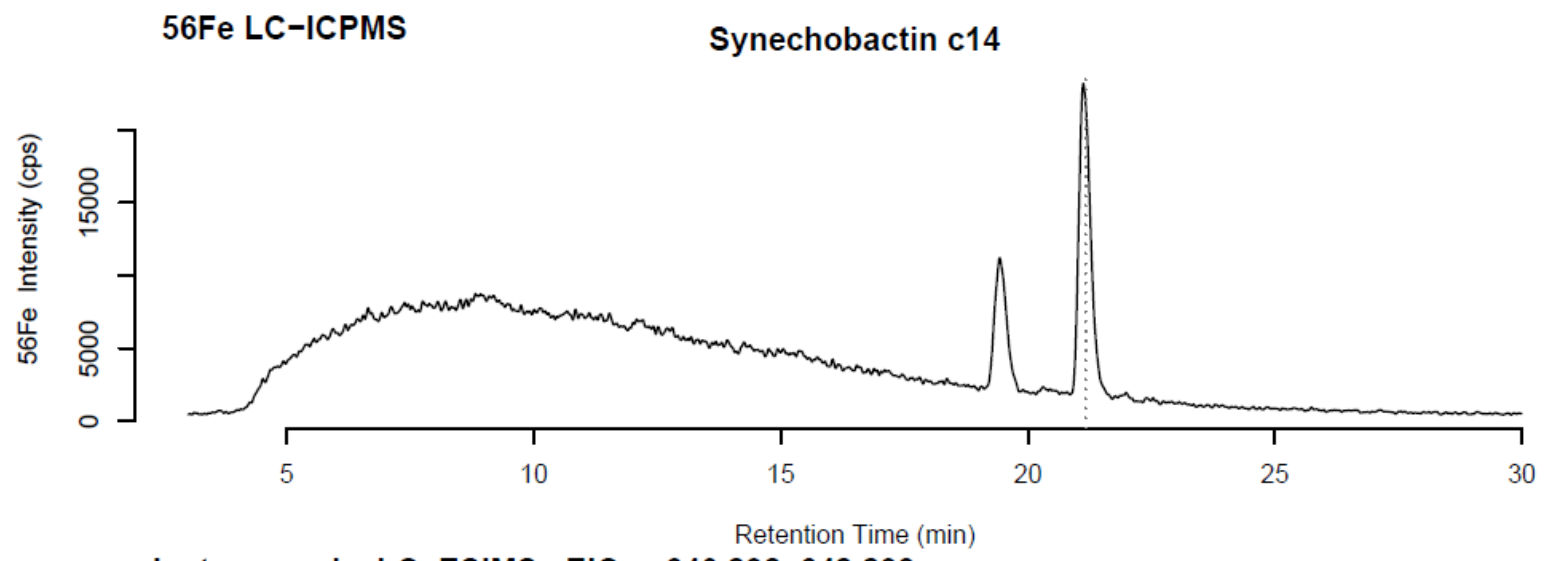

Isotope peaks LC-ESIMS. EIC $=640.298,642.293$

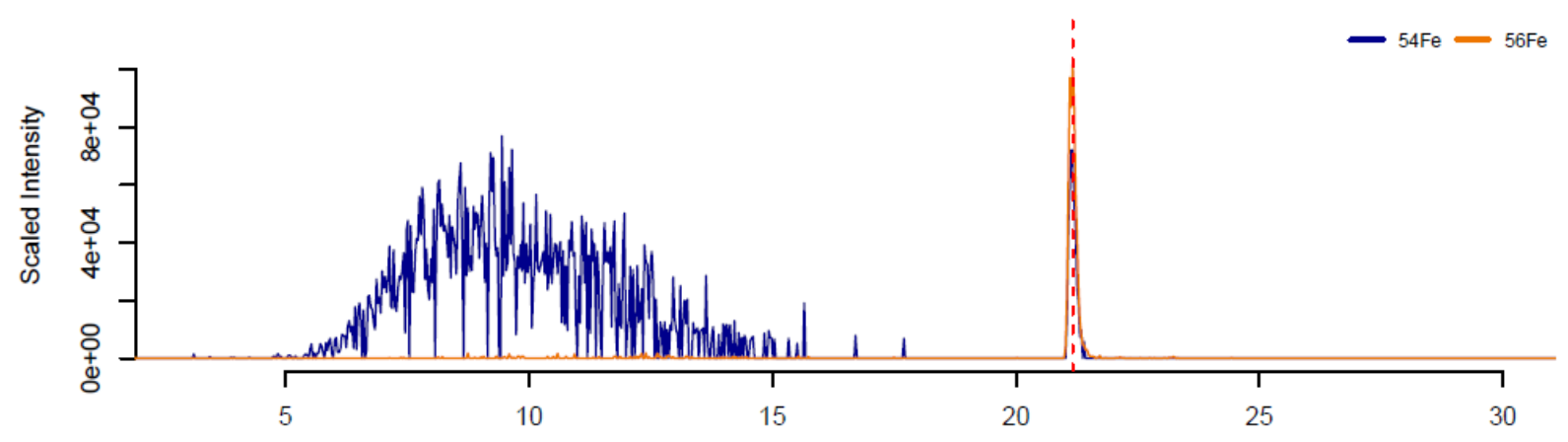

Retention Time (min)

MS2 fragmentation. Precursor $=642.29$ scan time $=21.05 \mathrm{~min}$

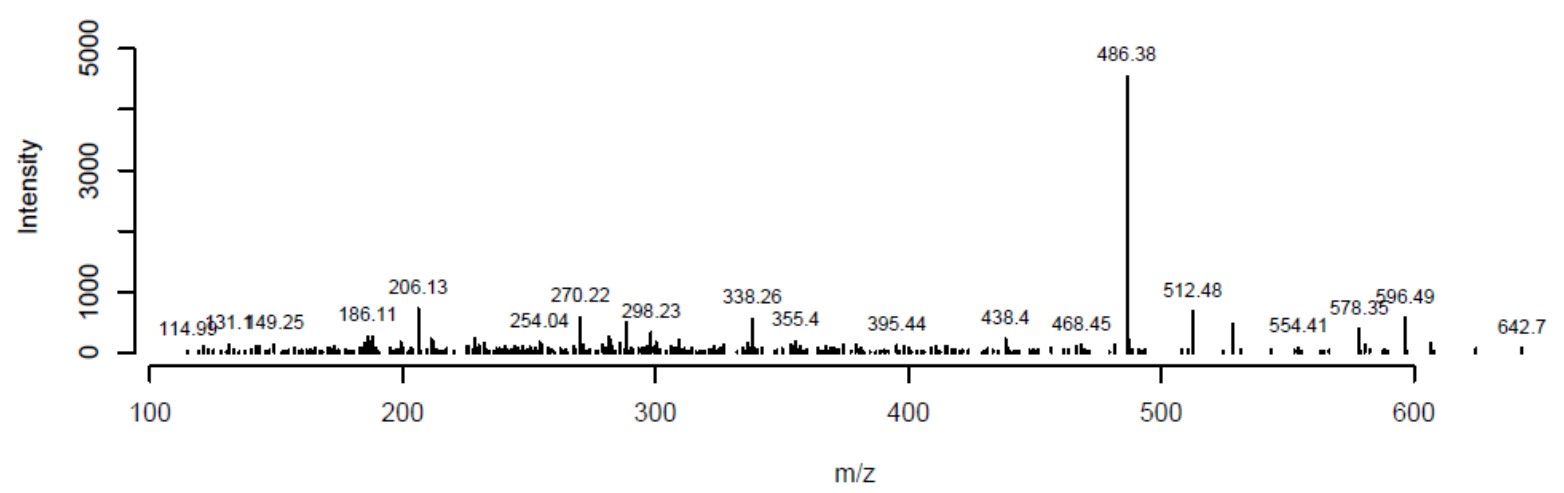

Figure A5.1-2: (top panel) 56Fe LC-ICPMS chromatogram (middle panel) LC-ESIMS extracted ion chromatograms for the $54 \mathrm{Fe}$ (blue) and $56 \mathrm{Fe}$ (orange) containing isotopologues $[\mathrm{M}+\mathrm{H}]^{+}$ ions. (bottom panel) MS2 fragmentation pattern for the $56 \mathrm{Fe}[\mathrm{M}+\mathrm{H}]^{+}$ion. Data collected from $60 \mathrm{~m}$ sample, Station 2. 


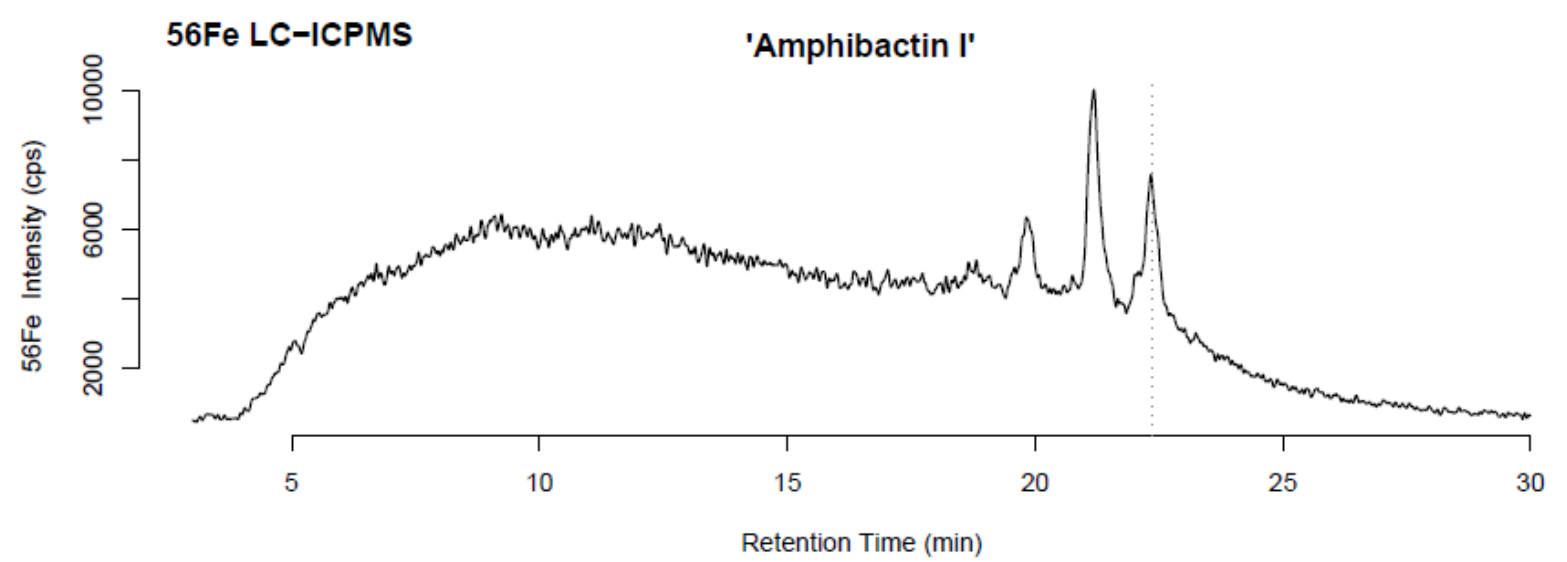

Isotope peaks LC-ESIMS. EIC $=937.467,939.462$

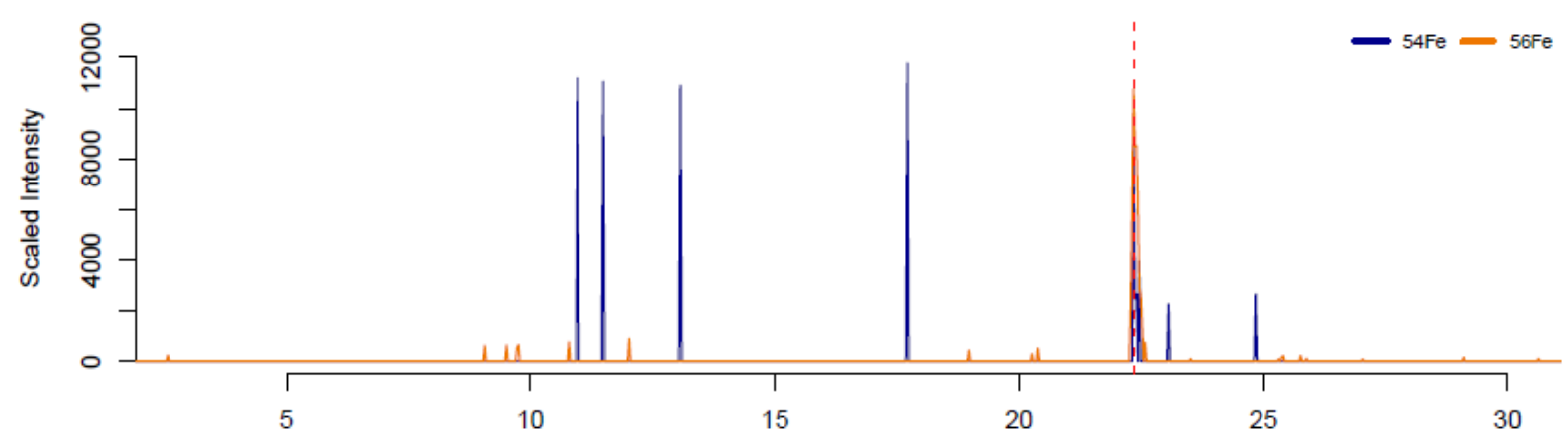

Retention Time (min)

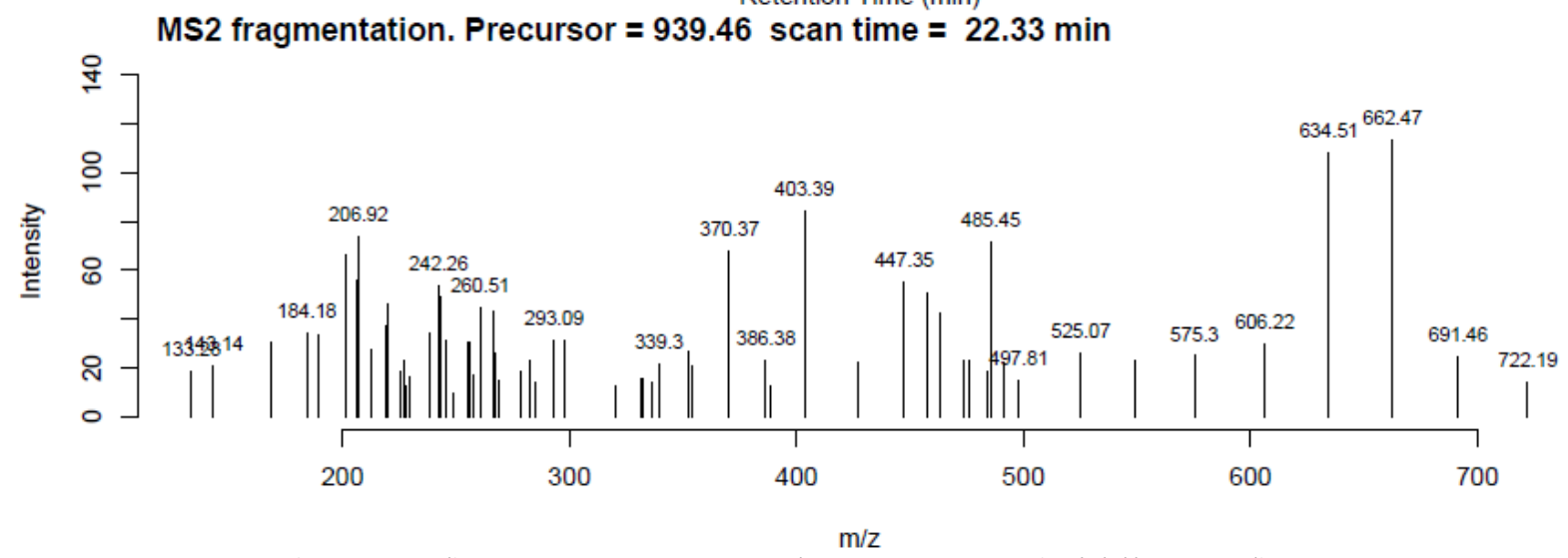

Figure A5.1-3: (top panel) 56Fe LC-ICPMS chromatogram (middle panel) LC-ESIMS extracted ion chromatograms for the $54 \mathrm{Fe}$ (blue) and $56 \mathrm{Fe}$ (orange) containing isotopologues $[\mathrm{M}+\mathrm{H}]^{+}$ ions. (bottom panel) MS2 fragmentation pattern for the $56 \mathrm{Fe}[\mathrm{M}+\mathrm{H}]^{+}$ion. Data collected from $81 \mathrm{~m}$ sample, Station 21. 


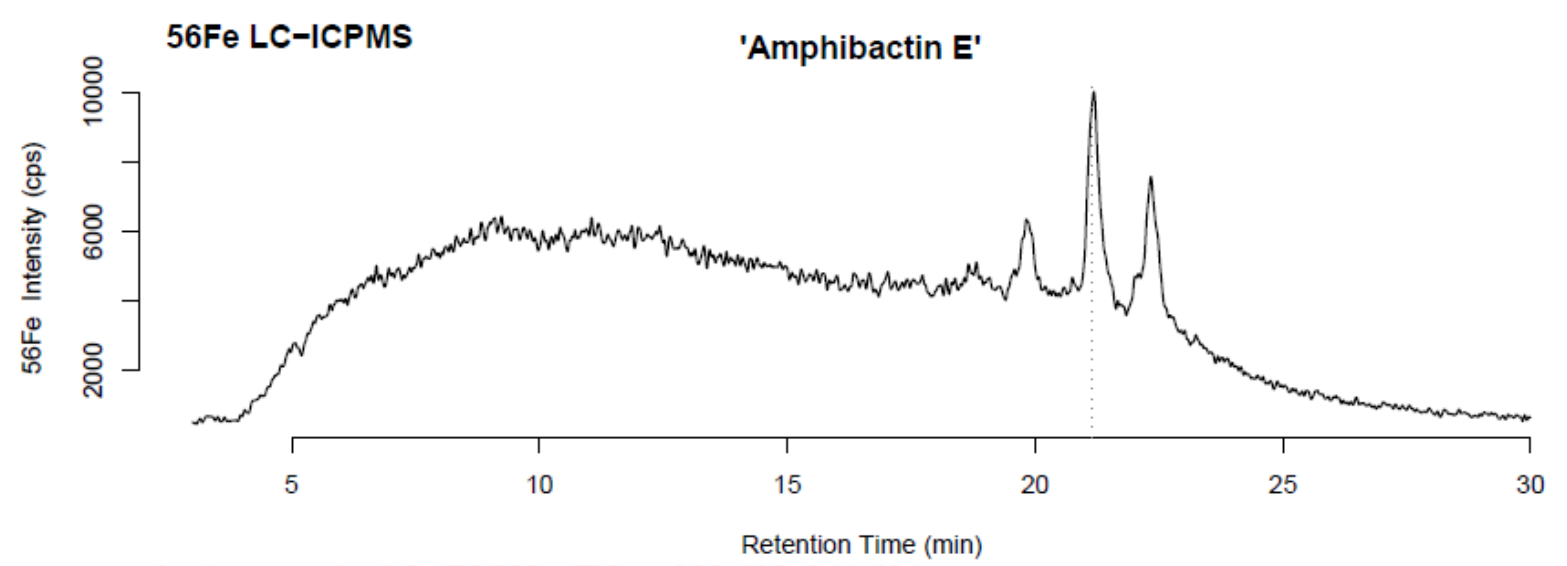

Isotope peaks LC-ESIMS. EIC $=909.435,911.431$

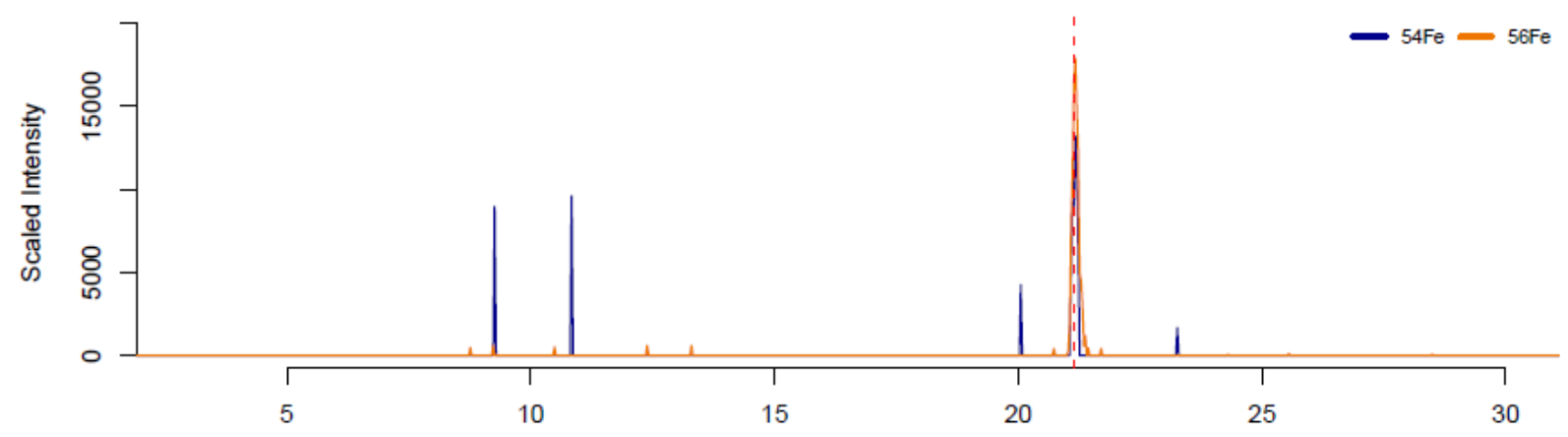

Retention Time (min)

MS2 fragmentation. Precursor $=911.43$ scan time $=21.21 \mathrm{~min}$

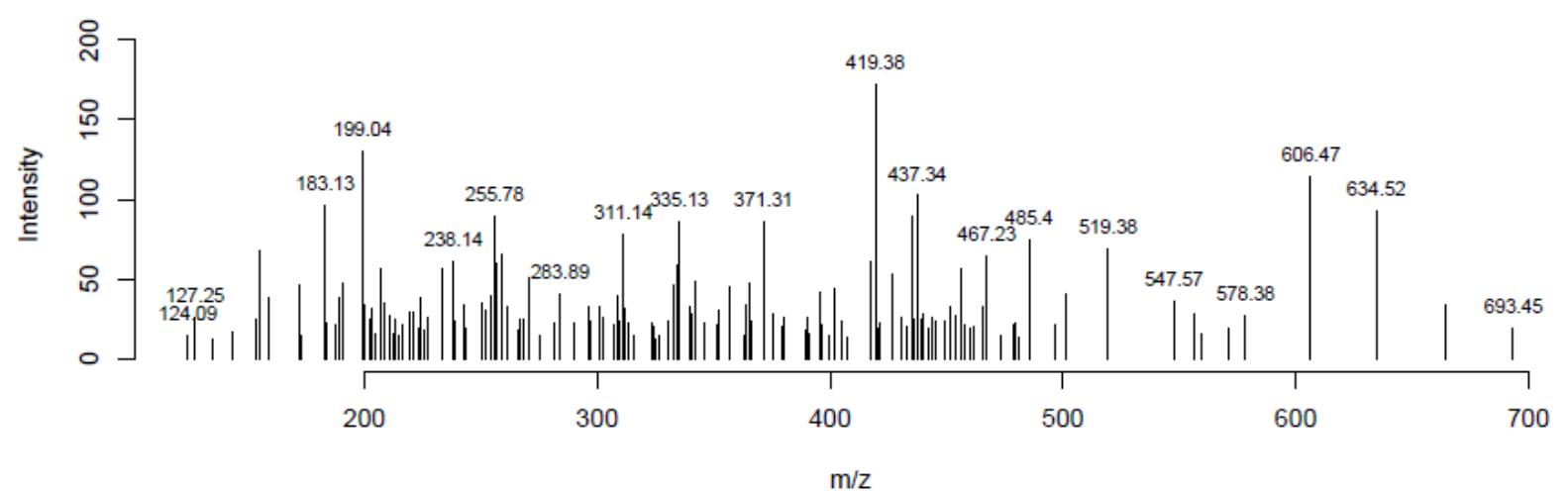

Figure A5.1-4: (top panel) 56Fe LC-ICPMS chromatogram (middle panel) LC-ESIMS extracted ion chromatograms for the $54 \mathrm{Fe}$ (blue) and $56 \mathrm{Fe}$ (orange) containing isotopologues $[\mathrm{M}+\mathrm{H}]^{+}$ ions. (bottom panel) MS2 fragmentation pattern for the $56 \mathrm{Fe}[\mathrm{M}+\mathrm{H}]^{+}$ion. Data collected from $81 \mathrm{~m}$ sample, Station 21. 


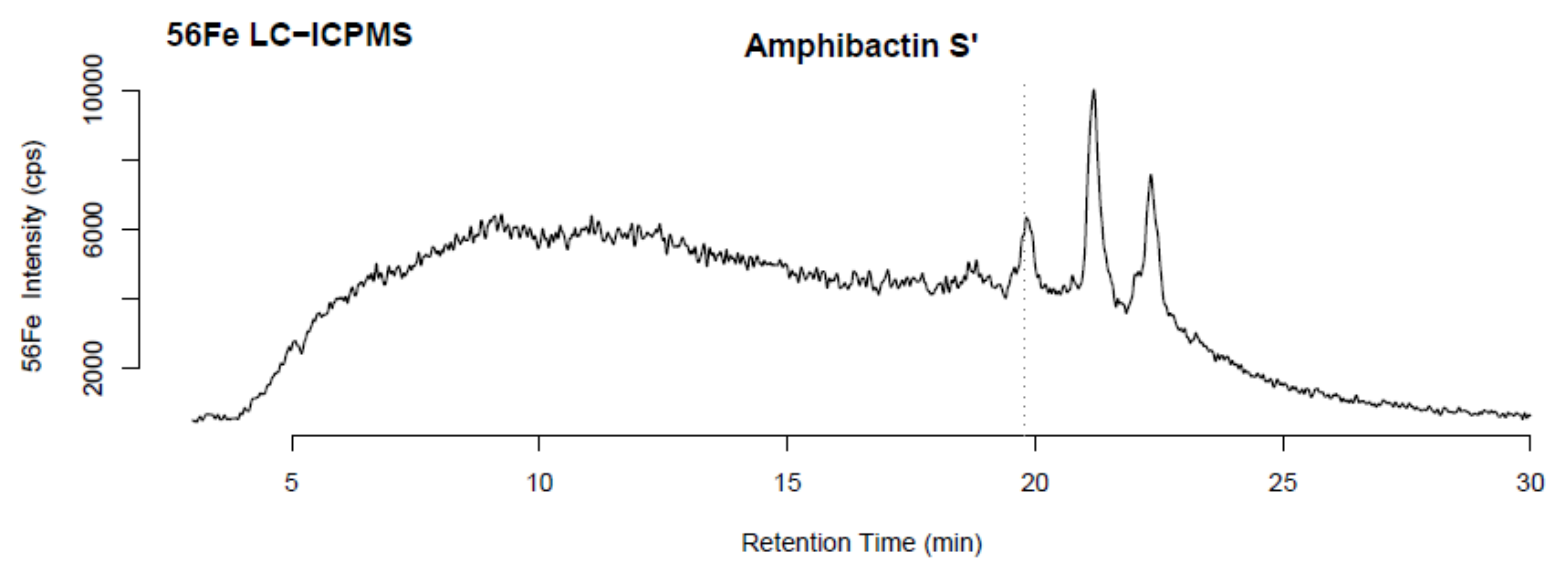

Isotope peaks LC-ESIMS. EIC $=\mathbf{8 8 1 . 4 0 4 , 8 8 3 . 3 9 9}$

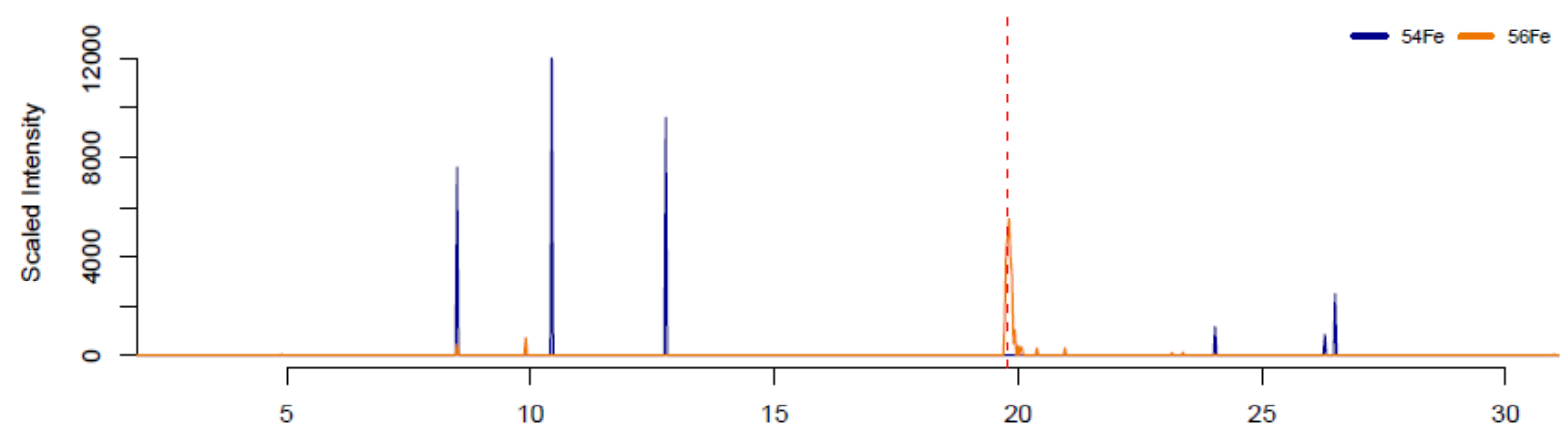

Retention Time (min)

MS2 fragmentation. Precursor $=883.4$ scan time $=19.82 \mathrm{~min}$

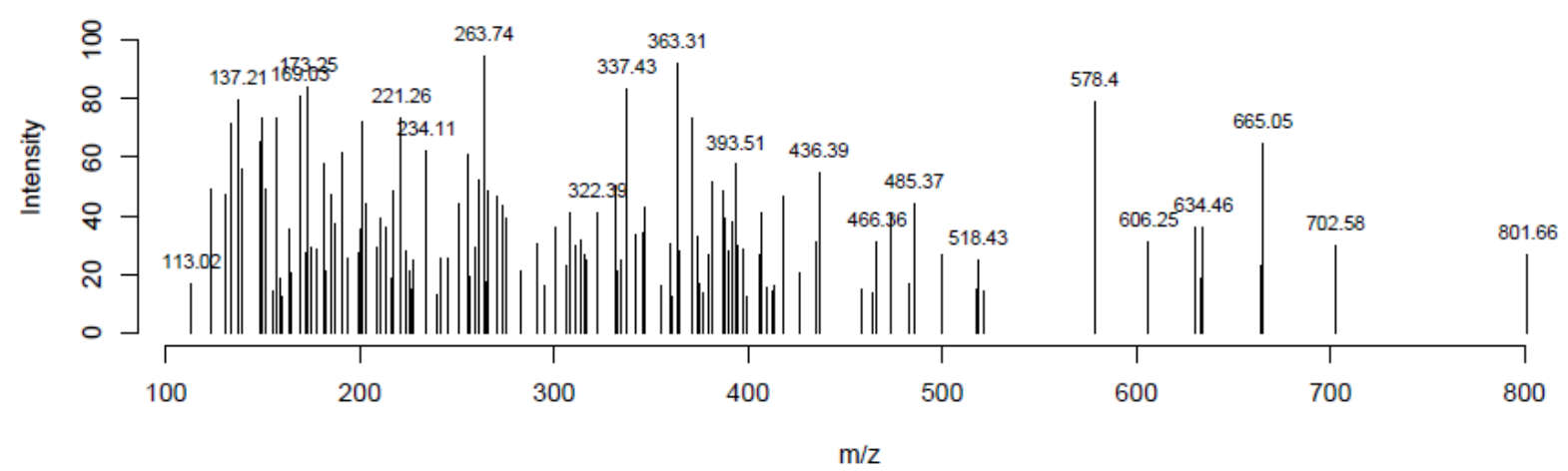

Figure A5.1-5: (top panel) 56Fe LC-ICPMS chromatogram (middle panel) LC-ESIMS extracted ion chromatograms for the $54 \mathrm{Fe}$ (blue) and $56 \mathrm{Fe}$ (orange) containing isotopologues $[\mathrm{M}+\mathrm{H}]^{+}$ ions. (bottom panel) MS2 fragmentation pattern for the $56 \mathrm{Fe}[\mathrm{M}+\mathrm{H}]^{+}$ion. Data collected from $81 \mathrm{~m}$ sample, Station 21. 


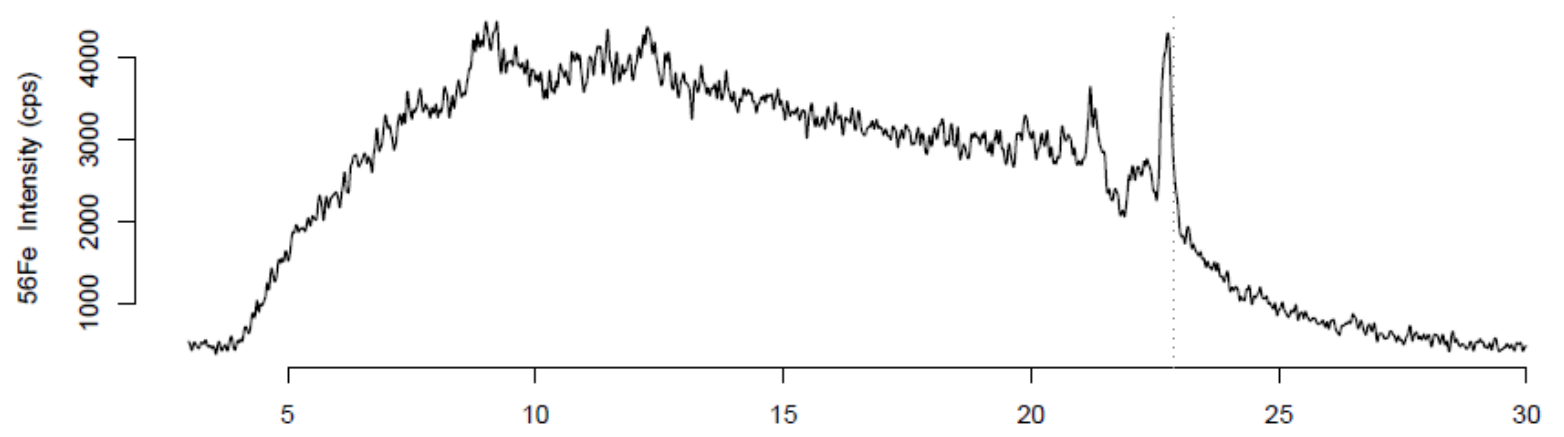

Retention Time (min)

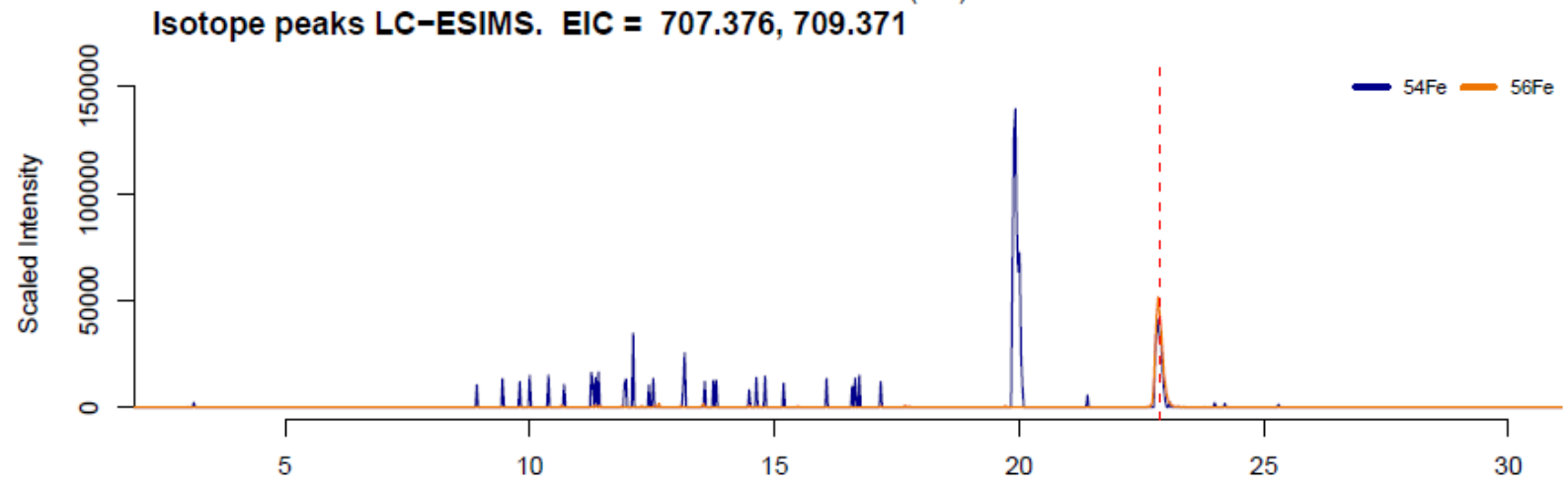

Retention Time (min)

MS2 fragmentation. Precursor $=709.37$ scan time $=22.76 \mathrm{~min}$

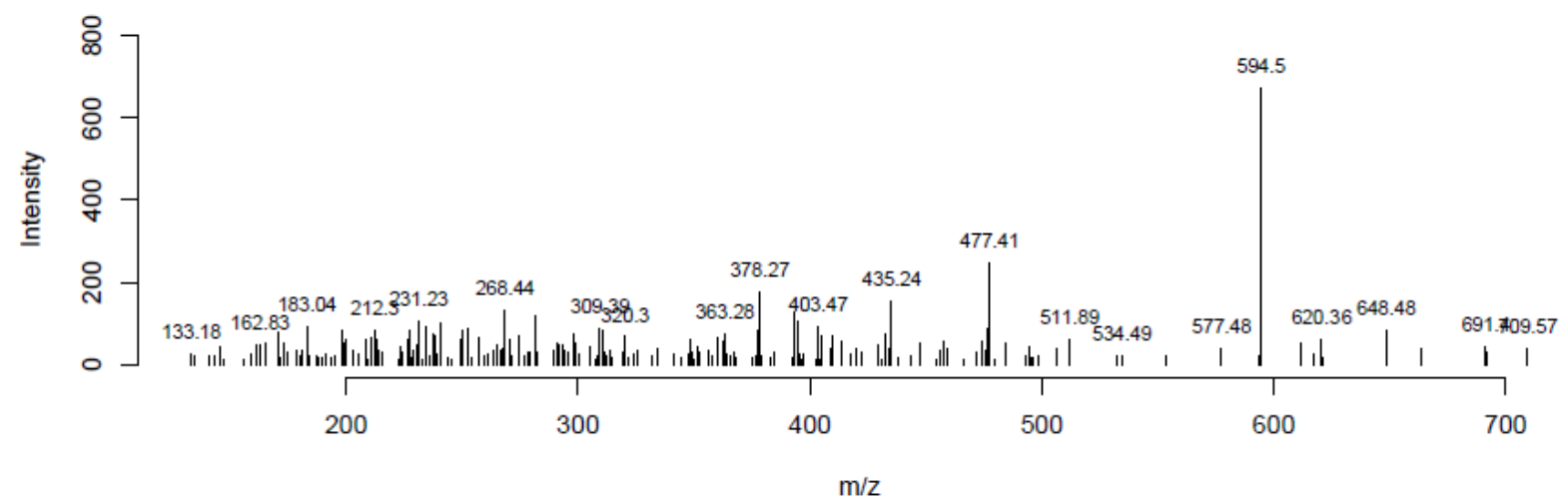

Figure A5.1-6: (top panel) 56Fe LC-ICPMS chromatogram (middle panel) LC-ESIMS extracted ion chromatograms for the $54 \mathrm{Fe}$ (blue) and $56 \mathrm{Fe}$ (orange) containing isotopologues $[\mathrm{M}+\mathrm{H}]^{+}$ ions. (bottom panel) MS2 fragmentation pattern for the $56 \mathrm{Fe}[\mathrm{M}+\mathrm{H}]^{+}$ion. Data collected from surface sample, transect 9-3. 


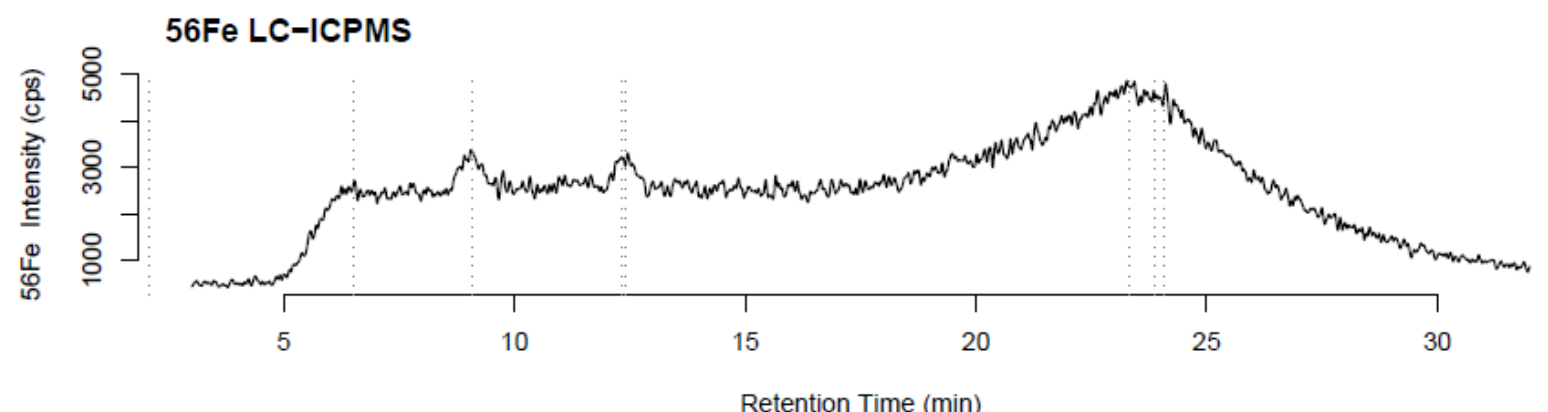

'Desferal, DFOB, Desferrioxamine B' Apo mass: LC-ESIMS. EIC = 561.361
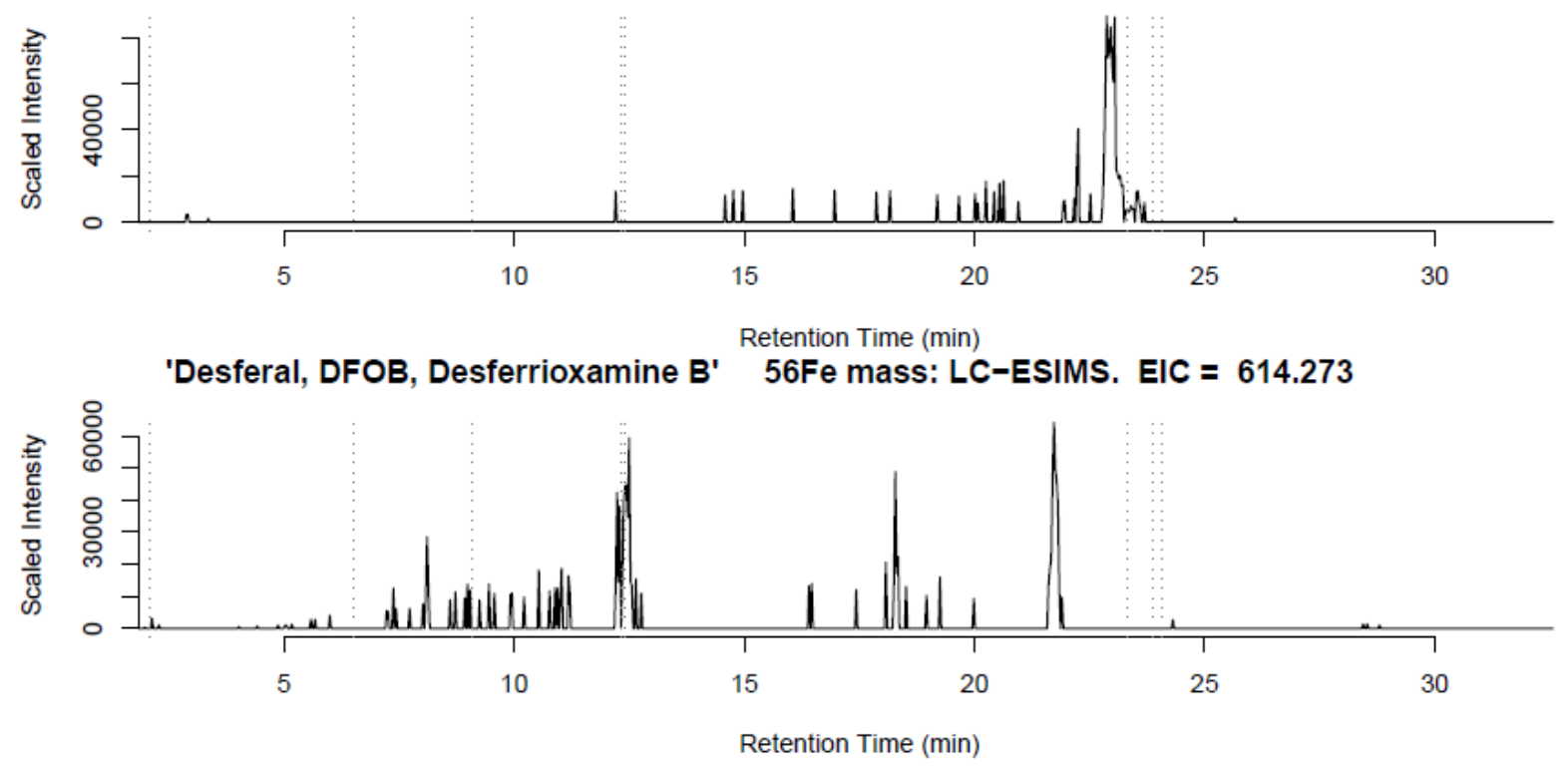

'Desferal, DFOB, Desferrioxamine B' 54Fe mass: LC-ESIMS. EIC = 612.277

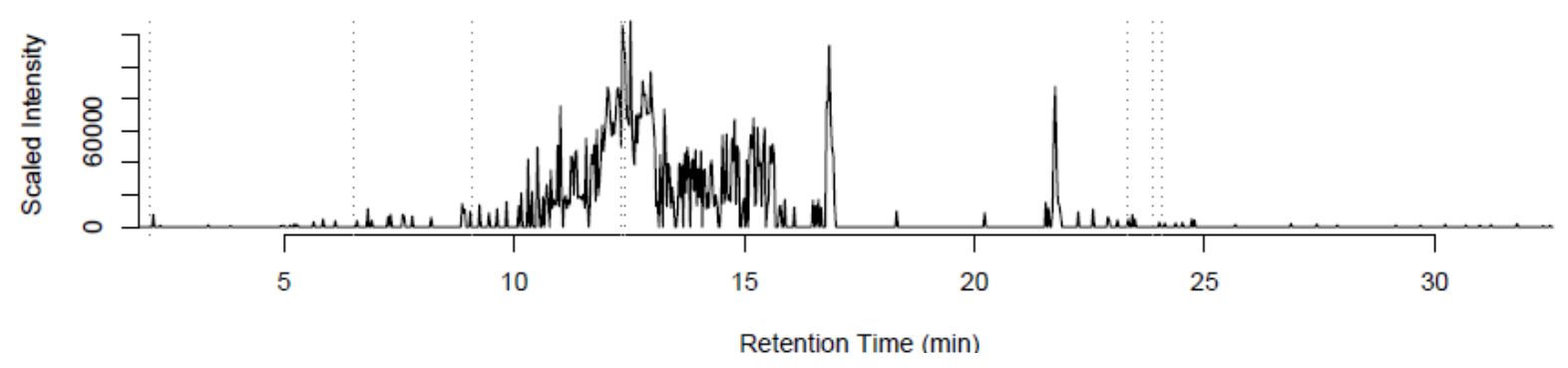

Figure A5.1-7: Putative identification of ferroxamine B. (top panel) 56Fe LC-ICPMS chromatogram (second panel) LC-ESIMS extracted ion chromatograms for the iron free (apo) mass, the $56 \mathrm{Fe}$ isotopologues $[\mathrm{M}+\mathrm{H}]^{+}$ion, and the $54 \mathrm{Fe}$ isotopologues $[\mathrm{M}+\mathrm{H}]^{+}$ion. Isobaric interferences at the mass of $54 \mathrm{Fe}$ ferrioxamine $\mathrm{B}$ make it difficult to confirm the identity of this compound. Data collected from the surface sample, station 2. 


\section{Appendix 5.2: Halogenated compounds in the upwelling community:}

In the surface of the wide shelf region, there is a source of halogenated compounds containing bromine and iodine. Numerous bromine containing compounds are highly abundant at $6 \mathrm{~m}$, while one of these compounds, eluting at $19 \mathrm{~min}$ is a dibromo compound that is also present at $20 \mathrm{~m}$. Diatoms are known to produce haloperoxidases that are capable of brominating other compounds within the water column, and it is possible that the nonspecific activity of these enzymes is responsible for generating these compounds.[Moore et al., 1996; Amin et al., 2012] However, it is unlikely for a dibromo compound to be produced by haloperoxidases, and it is more likely that this compound is produced by a more specific biosynthetic pathway.[Agarwal and Moore, 2014; Agarwal et al., 2014, 2015]

Several iodine containing compounds also appear in the surface and $20 \mathrm{~m}$ samples. It is possible that they are also produced by haloperoxidases, although the retention times and distribution of these iodine coumpounds are significantly different than their bromine counterparts. Alternatively, photochemistry has also been implicated in the abiotic formation of iodine compounds.[Carpenter, 2003; Méndez-Díaz et al., 2014] Due to the monoisotopic nature of iodine, determining their mass within the ESIMS data remains a challenge. 

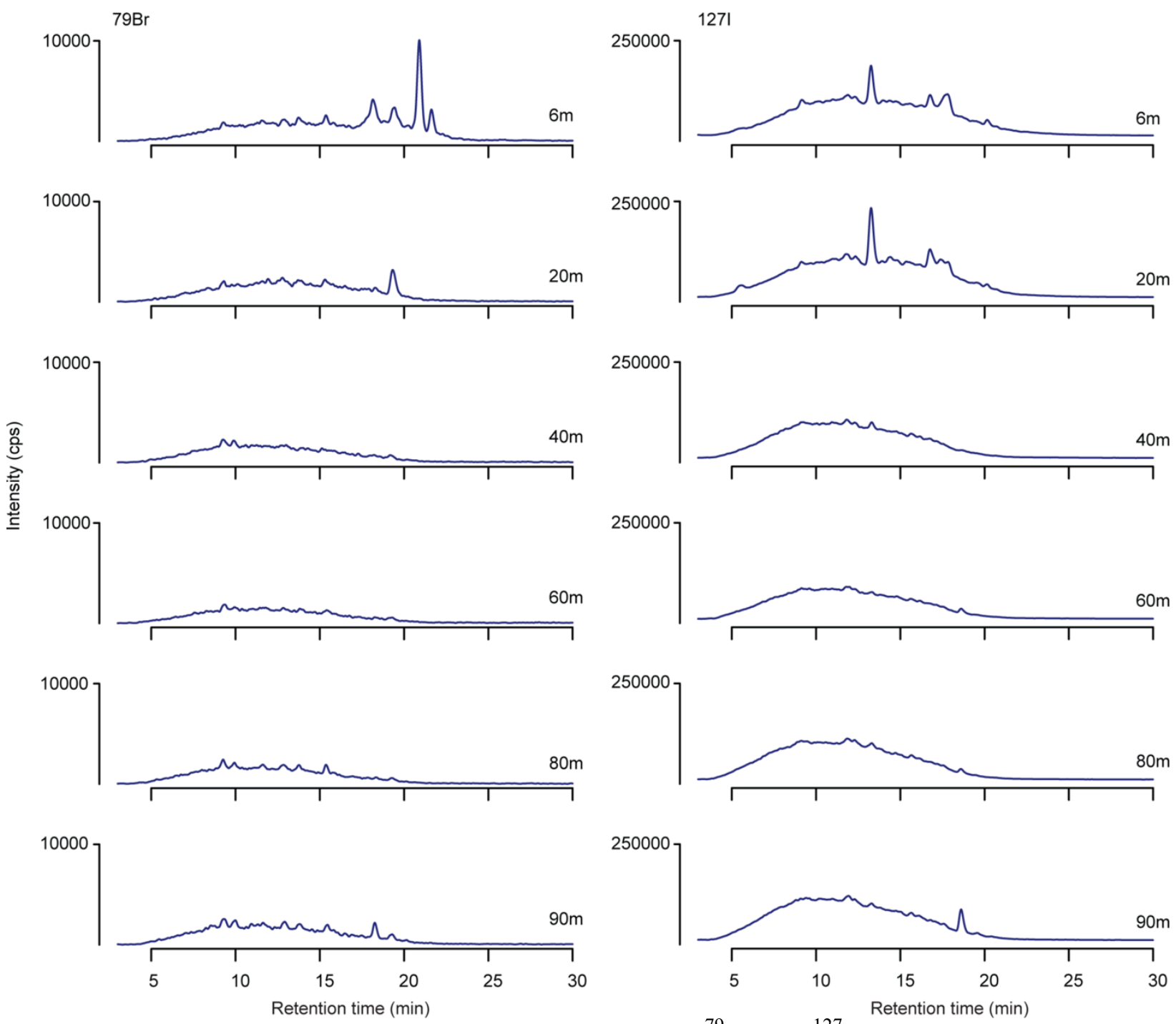

Figure A5.2-1: LC-ICPMS chromatograms for halogens $\left({ }^{79} \mathrm{Br}\right.$ and $\left.{ }^{127} \mathrm{I}\right)$ from a depth profile near the wide continental shelf (station 2). 
Table A5.2-1: Brominated compounds detected within the wide shelf surface waters

\begin{tabular}{lll}
\hline Retention Time $(\mathrm{min})$ & $79 \mathrm{Br} \mathrm{m} / \mathrm{z}$ & Identity \\
\hline 19.3 & 564.107 & dibromo, unknown \\
21.1 & 382.159 & Unknown \\
21.8 & 396.211 & Unknown
\end{tabular}




\title{
Chapter 6: Compound specific determination of ligand dissociation kinetics by isotopic exchange
}

\begin{abstract}
:
Our current understanding of iron speciation in the ocean is based on thermodynamic measurements of concentrations and stability constants at chemical equilibrium. Yet iron cycling is extremely dynamic, involving iron binding and dissociation from strong binding ligands, reduction and oxidation by photochemistry and biology, and uptake/release by cells and particles. Kinetic approaches are needed to evaluate the effect of organic ligands on these iron cycling processes. Several studies have investigated the rates of iron transformation between chemical species using competitive ligand exchange (CLE). CLE has yielded important information on bulk ligand association and dissociation rates and suggest that they are quite variable throughout the ocean. This variability in exchange kinetics is likely due to differences in the composition of the ligand pool. LC-ICPMS has begun to uncover the chemical identity of these components, and the next challenge is to connect them to the kinetic and thermodynamic iron cycling processes that occur in the ocean. Towards this goal, I developed a novel method for determining iron ligand dissociation rates by monitoring the isotopic exchange of natural ${ }^{56} \mathrm{Fe}$ with ${ }^{57} \mathrm{Fe}$ using LC-ICPMS. As a preliminary study, dissociation rates were determined for $2 \mathrm{nM}$ solutions of ferrichrome and ferrioxamine E. In seawater $(\mathrm{pH} \mathrm{8)}$, the dissociation of ferrichrome $\left(\mathrm{k}_{\mathrm{d}} 8.4 \times 10^{-8} \mathrm{~s}^{-1}\right)$ was faster than that of ferrioxamine $\mathrm{E}\left(\mathrm{k}_{\mathrm{d}} 3.4 \times 10^{-8} \mathrm{~s}^{-1}\right)$ although both rates were slow overall, with mean lifetimes $>135$ days for the iron-bound species. Rates for both compounds were $50 \%$ slower in $\mathrm{qH}_{2} \mathrm{O}$ compared with seawater, suggesting that cations in seawater accelerate dissociation. Isotope exchange experiments on organic extracts from natural seawater indicated that chromatographically unresolved dissolved organic matter (DOM) rapidly dissociated from iron while an unknown siderophore with m/z 709 dissociated within 4 days and amphibactins dissociated within 12 days. The fast dissociation rates in the concentrated organic extracts compared to dilute sewater are likely due to accelerated second order kinetic processes. These different dissociation rates reflect different iron binding strengths and indicate that isotopic labeling of iron-bound natural ligands within an organic extract requires long equilibration times.
\end{abstract}




\subsection{Introduction:}

Organic complexation controls the solubility, reactivity, and biological availability of many transition metals in the ocean, including $\mathrm{Fe}, \mathrm{Co}, \mathrm{Cu}, \mathrm{Ni}$, and $\mathrm{Zn}$. Since the discovery that metal concentrations limit biological productivity in large areas of open ocean[Martin et al., 1991, 1994; De Baar et al., 2005; Boyd et al., 2007], there has been significant interest in understanding the role of organic ligands and incorporating them into biogeochemical ocean models. Most of the work to characterize these ligands has focused on their concentrations and binding strengths. Electrochemical measurements have shed light on the distribution of these important organic ligands throughout the ocean by providing estimates of the total concentration and conditional stability constants of the compounds in seawater [Vraspir and Butler, 2009]. In the case of iron in particular, organic compounds complex over $99 \%$ of the dissolved metal [Rue and Bruland, 1995, 1997]. Organic ligands are responsible for maintaining dissolved iron stocks available for biological uptake that are well above the solubility of inorganic iron species in seawater (<80pM) [Kuma et al., 1996, 1998; Liu and Millero, 2002]. Competitive ligand exchange experiments have demonstrated that the concentrations and binding strengths for these compounds vary extensively throughout the ocean. Surface and coastal waters tend to have higher concentrations than the oligotrophic or deep ocean, suggesting that marine organic ligands have multiple important sources including biological productivity and terrestrial inputs. Furthermore, the conditional iron binding stability constants vary spatially and cover a wide range from 10.2-13.6 (log $\left.\mathrm{K}_{\text {cond }}\right)$ [Gledhill and Buck, 2012; Bundy et al., 2014].

The dynamic spatial and temporal variability in iron ligand composition is matched by the variability in other processes that affect iron cycling Cells take up and release iron and organic ligands as they grow and divide or break open from grazing or viral lysis [Boyd et al., 2010, 2015]. Photochemistry reduces Fe(III) to Fe(II) during the day in the surface ocean [Johnson et al., 1994; Kieber et al., 2005]. Atmospheric particle deposition adds new iron to the ocean during episodic events [Mahowald and Engelstaedter, 2009; Baker and Croot, 2010; Hayes et al., 2015]. These processes often occur on hourly, daily and monthly time scales. Therefore, determining the kinetics of iron exchange in seawater is essential for understanding biogeochemical iron cycling. A number of studies have addressed this question using 
competitive ligand exchange [Wu and Luther, 1995; Witter and Luther, 1998; Witter et al., 2000; Gerringa et al., 2007; Croot and Heller, 2012; Laglera and Filella, 2014]. These studies have determined the timescales of iron association and dissociation from both model and natural ligands. However, the widely varying exchange rates observed in these studies reflect an average value measure the exchange rates of individual components that make up the ligand pool and evaluate their respective contributions to iron cycling.

More recently, the diversity of iron binding ligands has been revealed by chromatography and mass spectrometry based approaches [Macrellis et al., 2001; Gledhill et al., 2004; Mawji et al., 2008; Velasquez et al., 2011]. These techniques are just beginning to reveal the diverse chemical composition of marine ligands (see chapters 4 and 5). They include siderophores, biomolecules that are used for the acquisition of iron under iron-stressed conditions, as well as chromatographically unresolved dissolved organic matter (DOM) that likely result from the degradation of biomolecules into refractory organic compounds with extreme structural variability. In other cases, the identity of these compounds is unknown. A major challenge that remains is linking the chemical/structural information obtained by LCMS based approaches to the bulk measurements acquired by competitive ligand exchange measurements. This requires new ways of determining physical parameters (e.g. binding strength or kinetic constants) of the compounds detected by LCMS that can be compared to those determined with competitive ligand exchange voltammetry experiments. Eventually, such information will lead to a better understanding of processes and rates such as biological uptake, reduction, precipitation, absorption/scavenging associated with specific compounds or compound classes that occur in the environment.

In this chapter, I introduce a novel approach for determining compound specific iron dissociation rates from a mixed ligand sample based on the exchange of naturally abundant ${ }^{56} \mathrm{Fe}$ with the rare ${ }^{57} \mathrm{Fe}$ isotope. This method provides a means of determining the dissociation rates of iron ligands under close-to-natural seawater conditions and ligand concentrations. Secondly, it provides a means to determine the rate at which iron isotopes exchange in concentrated organic extracts. This information is important for future studies that seek to label natural iron ligands with a rare 
or radioactive isotope for biological uptake studies or validation of ligand masses determined by electrospray ionization mass spectrometry.

\subsection{Exchange kinetics:}

Studying compound specific dissociation kinetics in a complex mixture requires a method that can independently resolve the amount of metal associated with each species. Chromatography coupled with mass spectrometry can achieve the compound specific detection, but is potentially subject to changes in speciation during the course of the chromatography (i.e. loss of metal from the organic ligand during chromatographic separation). To circumvent this issue, isotopic exchange can be used to determine dissociation kinetics. In this case, all organic ligands are saturated with a metal, and then a rare isotope of the same metal is added in large excess. Assuming that the association rate is much faster than dissociation, as is the case for strong iron ligands, the rate of isotope exchange is determined by the slow dissociation step.

In order to investigate relative binding strengths of ligands in the complex mixture, isotopically enriched iron was added in a large excess over ${ }^{56} \mathrm{Fe}$, stabilized in solution by citrate. Following the kinetic theory of trace metal complexation described by Hering and Morel (1990), for the overall reaction:

${ }^{56} \mathrm{FeL}+{ }^{57} \mathrm{Fe} \leftrightarrow{ }^{57} \mathrm{FeL}+{ }^{56} \mathrm{Fe}$

Iron exchange can proceed via a two-step disjunctive mechanism, in which an iron-ligand species dissociates, followed by complexation of a new iron atom. The disjunctive mechanism can be written as:

${ }^{56} \mathrm{FeL} \underset{k d i s^{\prime}}{\stackrel{k d i s}{\rightleftarrows}}{ }^{56} \mathrm{Fe}+\mathrm{L}$ 
${ }^{57} \mathrm{Fe}+\underset{\text { kdis }}{\stackrel{k^{2 d i s^{\prime}}}{\rightleftarrows}}{ }^{77} \mathrm{FeL}$

Where $\mathrm{k}_{\mathrm{dis}}$ and $\mathrm{k}_{\mathrm{dis}}$ ' are apparent disjunctive dissociation and association rates at a given $\mathrm{pH}$ and salinity.

Alternatively, the reaction can follow an adjunctive mechanism involving the formation of a ternary complex as an intermediate:

${ }^{56} \mathrm{FeL}+{ }^{57} \mathrm{Fe}^{\prime} \underset{k_{\text {adj } j, F e}}{\stackrel{k_{\text {adj } j, F e}}{\rightleftarrows}}{ }^{57} \mathrm{FeL}+{ }^{56} \mathrm{Fe}^{\prime}$

Or it could take place as an adjunctive dissociation involving slow competition from another ligand in solution (Ln), such as citrate (4), followed by a fast second order mechanism of iron association (2).

${ }^{56} \mathrm{FeL}+\mathrm{Ln} \underset{k_{a d j, L n}{ }^{\prime}}{\stackrel{k_{a d j, L n}}{\rightleftarrows}}{ }^{56} \mathrm{FeLn}+L$

When an isotopically enriched ${ }^{57} \mathrm{Fe}$ spike is added to a sample containing a strong ligand $\mathrm{L}$, the concentration of ${ }^{56} \mathrm{FeL}$ will decrease as the ligand exchanges ${ }^{56} \mathrm{Fe}$ for ${ }^{57} \mathrm{Fe}$. This process depends on the mole fraction of ${ }^{56} \mathrm{Fe} / \mathrm{Fe}_{\text {total }}\left(\mathrm{f}_{56 \mathrm{Fe}}\right)$ of the ambient iron pool that is exchanging with $\mathrm{L}$. When $\left[{ }^{57} \mathrm{Fe}^{\prime}\right] \gg>\left[{ }^{56} \mathrm{Fe}\right.$ '] (i.e. $f_{56 \mathrm{Fe}}$ is small) and $\left[{ }^{56} \mathrm{FeL}\right] \gg>\left[{ }^{57} \mathrm{FeL}\right]$ (i.e. the initial reaction rate is considered), the dissociation of ${ }^{56} \mathrm{FeL}$ is much faster than its formation and the back reaction of ${ }^{57} \mathrm{FeL}$ to ${ }^{56} \mathrm{FeL}$ can be ignored. We can describe the loss rate of ${ }^{56} \mathrm{FeL}$ by the following equation derived from equations (1), (3), and (4):

$\frac{d 56 \mathrm{FeL}}{d t}=\left(1-f_{56 \mathrm{Fe}}\right)\left(-k_{d i s}\left[{ }^{56} \mathrm{FeL}\right]-k_{a d j, \mathrm{Fe}}\left[{ }^{56} \mathrm{FeL}\right]\left[\mathrm{Fe}^{\prime}\right]-k_{a d j, \mathrm{Ln}}\left[{ }^{56} \mathrm{FeL}\right][\mathrm{Ln}]\right)$ 
The first term $\left(1-\mathrm{f}_{56 \mathrm{Fe}}\right)$ takes into account the events where ${ }^{56} \mathrm{Fe}$ is exchanged for another ${ }^{56} \mathrm{Fe}$ rather than ${ }^{57} \mathrm{Fe}$. In the case where the concentration of the added iron isotope spike is much greater than the concentration of ${ }^{56} \mathrm{FeL}$, the dissociation of ${ }^{56} \mathrm{FeL}$ will have little effect on $\mathrm{f}_{56 \mathrm{Fe}}$. Thus, $\mathrm{f}_{56 \mathrm{Fe}}$ can be assumed to be constant with time and equal to $\mathrm{f}_{56 \mathrm{Fe}}$ of the ${ }^{57} \mathrm{Fe}$ enriched spike.

Assuming steady state for [Fe'] and [Ln], a pseudo-first order kinetic equation can be written to describe the overall kinetics of Fe dissociation:

$\frac{d 56 F e L}{d t}=\left(1-f_{56 F e}\right)\left(-k_{d i s}-k_{a d j, F e}\left[F e^{\prime}\right]-k_{a d j, L n}[\mathrm{Ln}]\right)\left[{ }^{56} F e L\right]$

Where we can define $\mathrm{k}_{\mathrm{d}}$ as the apparent dissociation rate for the metal ligand complex:

$\frac{d 56 F e L}{d t}=-\left(1-f_{56 F e}\right) k_{d}\left[{ }^{56} \mathrm{FeL}\right]$

$k_{d}=\left(k_{d i s}+k_{a d j, F e}\left[F e^{\prime}\right]+k_{a d j, L n}[\mathrm{Ln}]\right)$

Note that additional adjunctive terms can be added to the pseudo first order rate constant if higher order processes or more than one metal or ligand may be involved in additional dissociative pathways.

Equation (8) illustrates that the observed dissociation rate $\left(\mathrm{k}_{\mathrm{d}}\right)$ determined using isotope exchange with ${ }^{57} \mathrm{Fe}$-citrate is likely faster than the rates in natural seawater due to the addition of adjunctive pathways for iron dissociation from the added metal and citrate. However, these adjunctive mechanisms could be constrained in future studies by measuring dissociation rates with different metal/ligand concentrations added. Furthermore, fast equilibrating protonated species and side reaction coefficients could be expressed explicitly in these equations to compare studies at different $\mathrm{pH}$ or ionic strength. For now, the pseudo-first order rates are sufficient to determine upper bounds on the dissociation rate of metals and start to investigate binding strength differences between components. 
The integrated form of the pseudo-first order equation (7) yields:

$\left[{ }^{56} \mathrm{FeL}\right]=\left[{ }^{56} \mathrm{FeL}\right]_{\text {initial }} * e^{-(1-f 56 \mathrm{Fe}) k_{d} t}$

Dividing (9) by the total ligand concentration $\left[\mathrm{L}_{\text {total }}\right]$ gives:

$\frac{{ }^{56} \mathrm{FeL}}{\text { Ltotal }}=\left[\frac{{ }^{56} \mathrm{FeL}}{\text { Ltotal }}\right]_{\text {initial }} * e^{-(1-f 56 \mathrm{Fe}) k_{d} t}$

In the described system, where iron ligands are strong and specific, it can be assumed that $\mathrm{L}_{\text {total }}=$

${ }^{56} \mathrm{FeL}+{ }^{57} \mathrm{FeL}$. Thus, $\mathrm{k}_{\mathrm{d}}$ can be calculated from a logarithmic plot of ${ }^{56} \mathrm{FeL} /\left({ }^{56} \mathrm{FeL}+{ }^{57} \mathrm{FeL}\right)$ over time. The determination of the isotopic ratio ${ }^{56} \mathrm{FeL} /\left({ }^{56} \mathrm{FeL}+{ }^{57} \mathrm{FeL}\right)$ by LC-ICPMS is a robust measurement that is not impacted by iron loss during extraction or chromatography, and does not vary with changes in instrument sensitivity. Although mass biases do affect isotopic ratios determined by LC-ICPMS, these factors are very small compared to the changes that are measured in this study.

\subsection{Methods:}

\subsubsection{Materials and reagents:}

High purity water $\left(18.2 \mathrm{MO} \mathrm{cm}, \mathrm{qH}_{2} \mathrm{O}\right)$ and LCMS grade methanol $(\mathrm{MeOH})$ were used in this study. The methanol was redistilled in a polytetrafluoroethylene (PTFE) still to remove the iron blank, LCMS grade ammonium formate (Optima, Fisher scientific) was used in chromatographic solvents. Polycarbonate carboys and PTFE tubing for sample collection and solid phase extraction (SPE) were soaked overnight in $0.1 \%$ detergent (Citranox), rinsed with $\mathrm{qH}_{2} \mathrm{O}$, and soaked for 1 day in $1 \mathrm{~N} \mathrm{HCl}$ (trace metal grade, Fisher Scientific) before a final $\mathrm{qH}_{2} \mathrm{O}$ rinse. Stock solutions of ferrichrome, ferrioxamine E and cyanocobalamin (Sigma Aldrich) were prepared in $\mathrm{qH}_{2} \mathrm{O}$. To prepare the ${ }^{57} \mathrm{Fe}$ citrate stock solution, ${ }^{57} \mathrm{Fe}$ oxide (Cambridge Isotope 
Laboratories) was dissolved in concentrated $\mathrm{HCl}$ (trace metal grade), and was diluted to $4 \mu \mathrm{M} \mathrm{Fe}$ in a $1 \mathrm{mM}$ citrate solution prepared from sodium citrate dihydrate (Fisher scientific) in $\mathrm{qH}_{2} \mathrm{O}$.

\subsubsection{Sample collection:}

The sample used for the natural iron exchange rate experiment was obtained from the California current system in July 2014 on the IRNBRU cruise from $39.43^{\circ} \mathrm{N}, 124.54^{\circ} \mathrm{W}$. A volume of $20 \mathrm{~L}$ was collected from $3 \mathrm{~m}$ depth while transiting using a trace metal clean GeoFish sampling system and filtered through a $0.2 \mu \mathrm{m}$ polyethersulfone capsule filter into a $20 \mathrm{~L}$ polycarbonate carboy. Organic compounds were extracted from the seawater onto solid phase extraction resin columns $(1 \mathrm{~g}, \mathrm{ENV})$ at a rate of $15 \mathrm{~mL} / \mathrm{min}$ using a peristaltic pump equipped with polytetraflouroethylene (PTFE) and platinum-cured silicone tubing. Prior to use, the columns were primed with $5 \mathrm{~mL}$ $\mathrm{MeOH}$, rinsed with $10 \mathrm{~mL} \mathrm{pH} 2 \mathrm{qH}_{2} \mathrm{O}$ (acidified with trace metal grade $\mathrm{HCl}$ ), and conditioned

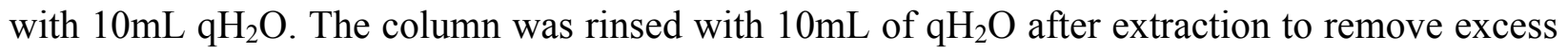
salt and was frozen onboard. The column was thawed and eluted with $6 \mathrm{~mL} \mathrm{MeOH}$ and added $10 \mu \mathrm{L}$ of a $50 \mu \mathrm{M}$ cycanocobalamin stock solution as an internal standard. This extract was concentrated to $2 \mathrm{~mL}$ under a stream of $\mathrm{N}_{2}$ gas.

\subsubsection{Fe exchange from model compounds under marine conditions:}

Two solutions containing $2 \mathrm{nM}$ each of ferrioxamine $\mathrm{E}$, ferrichrome, and cyanocobalamin (added to monitor ICPMS sensitivity) were prepared in two $60 \mathrm{~mL}$ PTFE bottles, one containing $50 \mathrm{~mL}$ $\mathrm{qH}_{2} \mathrm{O}$ and another containing $50 \mathrm{~mL}$ filtered seawater collected with a trace metal clean pump from the South Pacific Ocean. To each bottle, $1 \mathrm{~mL}$ of a ${ }^{57} \mathrm{Fe}$ citrate stock solution $(5 \mathrm{mM}$ sodium citrate dihydrate plus $4 \mu \mathrm{M}$ of a ${ }^{57} \mathrm{Fe}$ stock solution) was added. The solutions were filter sterilized through a $0.2 \mu \mathrm{M}$ polyethersulfone syringe filter (Millipore) into microwave sterilized polycarbonate bottles and incubated in the dark. $f_{56 \mathrm{Fe}}$ for the bulk solution was measured by ICPMS to be 0.11 . For each time point, $4 \mathrm{~mL}$ of each sample was passed through a $100 \mathrm{mg}$ C18 SPE column (Agilent Bond Elut) that had been primed with $\mathrm{MeOH}$ and rinsed with $\mathrm{qH}_{2} \mathrm{O}$. After extracting the compounds, the columns were rinsed with $1 \mathrm{~mL} \mathrm{qH}_{2} \mathrm{O}$ to remove salts and excess

${ }^{57} \mathrm{Fe}$ citrate, which is not captured by the column, and were stored at $-20^{\circ} \mathrm{C}$ until analyzed to 
prevent further isotopic exchange between the trapped iron species. Time points were collected over the course of 235 days. Samples were thawed and eluted with $0.8 \mathrm{~mL}$ of $\mathrm{MeOH}$. The solvent was removed by vacuum centrifugation (Savant speedvac), and samples were brought up in a final volume of $150 \mu \mathrm{L}$ with $\mathrm{qH}_{2} \mathrm{O}$. All analysis were made within one day to minimize the chance for continued iron exchange between eluted species.

Organic extracts were separated on a bioinert high pressure liquid chromatography (HPLC) system (Dionex Ultimate 3000) fitted with a C18 column (Hamilton, 2.1x150mm, $3 \mu \mathrm{m}$ particle size) and polyetheretherketone (PEEK) tubing and connectors. The sample was eluted with (A) $5 \mathrm{mM}$ aqueous ammonium formate and (B) $5 \mathrm{mM}$ ammonium formate in distilled $\mathrm{MeOH}$ using a 12 minute gradient from $10-60 \% \mathrm{~B}$, followed by isocratic elution at $60 \% \mathrm{~B}$ for 5 minutes at a flow rate of $0.2 \mathrm{~mL} / \mathrm{min}$. $50 \mu \mathrm{L} / \mathrm{min}$ of the eluent flow was directed to a quadruople ICPMS (Thermo iCAPq) by a post column PEEK tee. $\mathrm{O}_{2}$ gas $(25 \mathrm{~mL} / \mathrm{min})$ was added to the plasma to completely oxidize the organic solvent. Data was collected in kinetic energy discrimination mode monitoring ${ }^{56} \mathrm{Fe},{ }^{57} \mathrm{Fe}$, and ${ }^{59} \mathrm{Co}$ with a dwell time of 0.1 seconds using $4.5 \mathrm{~mL} / \mathrm{min} \mathrm{He}$ as a collision gas to remove the $\mathrm{ArO}^{+}$interference on ${ }^{56} \mathrm{Fe}$. Chromatographic peak areas were determined using a house made script in R. A linear regression was fit to a plot of $\ln \left({ }^{56} \mathrm{Fe} /\left({ }^{56} \mathrm{Fe}+{ }^{57} \mathrm{Fe}\right)\right)$ versus time for each compound and treatment. Values of $\mathrm{k}_{\mathrm{d}}$ presented in Table 1 were calculated from the slope coefficient and the standard error of the slope.

\subsubsection{Fe exchange of natural organic ligands:}

To investigate $\mathrm{Fe}$ exchange by natural organic ligands from seawater, $40 \mu \mathrm{L}$ of the ${ }^{57} \mathrm{Fe}$ citrate stock solution was mixed with $160 \mu \mathrm{L}$ of the concentrated organic extract from the California coast in a $250 \mu \mathrm{L}$ autosampler vial insert (Agilent Scientific). This sample was analyzed by LCICPMS after incubating at $25^{\circ} \mathrm{C}$ for 30 minutes, 4 days, and 10 days using a bioinert liquid chromatography system (Agilent 1260 Series) and a quadrupole ICPMS as described above, but using a 20 minute gradient from $5-95 \% \mathrm{~B}$, followed by a 10 minute isocratic elution at $95 \% \mathrm{~B}$. ${ }^{56} \mathrm{Fe},{ }^{57} \mathrm{Fe}$ and ${ }^{59} \mathrm{Co}$ were monitored with dwell times of 0.05 seconds. 


\subsection{Results and discussion:}

\subsubsection{Fe exchange for model compounds under marine conditions:}

The exchange kinetics of the model siderophores ferrichrome and ferrioxamine E were studied in both seawater $(\mathrm{pH} 8)$ and $\mathrm{qH}_{2} \mathrm{O}(\mathrm{pH} 5)$, in order to compare to previous studies on these ligands [Witter et al., 2000]. The ideal experiment for determining environmentally relevant dissociation rates would be conducted under conditions that represent seawater ionic strength, $\mathrm{pH}$, and ligand concentrations so that the resulting apparent dissociation rates would inform rates of iron cycling in the marine environment. LC-ICPMS can be used to monitor ligand isotope exchange under these conditions by including an extraction step to preconcentrate the organic ligands of interest (Fig. 6.1, 6.2). Although the ${ }^{57} \mathrm{Fe}$ spike was associated with a 100 -fold excess of citrate as a stabilizing ligand, citrate has a weak conditional stability constant relative to the model siderophores. Thus, the excess citrate maintained a high concentration of soluble iron but had little impact on the competition for iron bound by strong natural ligands.

Samples were collected at time points spanning 235 days by extracting the siderophores from the solution onto a $\mathrm{C} 18$ resin column, removing excess Fe-citrate and preventing continued isotopic exchange. Although care was taken to ensure no further isotope exchange at sampling time points and during the chromatography, iron may still dissociate from the ligands during sample preparation, and there is likely a mass dependent isotopic fractionation associated with this. However, these fractionation effects are much smaller than the analytical uncertainty of the iron measurements in this study (5-10\%) and the large isotopic changes that were measured from the

${ }^{57}$ Fe enriched samples. Furthermore, the use of isotopic ratios to determine metal exchange rates effectively eliminates the effect of changes in ICPMS signal intensity over the course of analysis.

Using this method, dissociation rates for ferrichrome and ferrioxamine $\mathrm{E}$ were determined in both $\mathrm{qH}_{2} \mathrm{O}$ and seawater (Fig. 6.3, Table 1). The dissociation of ferrichrome was faster than that of ferrioxamine $\mathrm{E}$ under both conditions, which is consistent with the higher affinity of ferrioxamine $\mathrm{E}$ for iron [Raymond et al., 1984]. The $\mathrm{k}_{\mathrm{d}}$ values obtained in seawater are in good agreement with previously published values from Witter et al., 2000 (shown in Table 1), which 
used electrochemical methods to monitor the competitive ligand exchange rate between 1nitroso-2-naphthol $(1 \mathrm{~N} 2 \mathrm{~N})$ and iron bound to individual siderophores in seawater. With this method, the dissociation constant is determined by dividing the observed dissociation rate (which is likely driven by second order adjunctive kinetics in those experiments) by the concentration of the added ligand. The competitive ligand exchange approach from these previous studies [ $W u$ and Luther, 1995; Witter et al., 2000] measured the pseudo first order dissociation constant of the siderophore at the experimental $1 \mathrm{~N} 2 \mathrm{~N}$ concentration by dividing the observed dissociation rate by the $1 \mathrm{~N} 2 \mathrm{~N}$ concentration, rather than the true first order disjunctive kinetic constant. The isotopic exchange approach described here represents an upper bound on environmental exchange kinetics, because it assumes that the adjunctive exchange pathways introduced by the addition of iron and citrate are negligible. Despite these differences, the close agreement between the $k_{d}$ values determined by the competitive ligand exchange approach and the isotopic exchange approach is encouraging. In future studies, it will be possible to separate the adjunctive and disjunctive rates of iron isotope exchange by measuring observed rates at multiple citrate concentrations.

For both siderophores, faster dissociation kinetics were observed in seawater at $\mathrm{pH} 8$ compared with $\mathrm{qH}_{2} \mathrm{O}$ at $\mathrm{pH}$ 5. This is consistent with the weaker stability constants of siderophores in seawater versus freshwater [Gledhill and Buck, 2012]. One possibility is that the lower $\mathrm{pH}$ in the $\mathrm{qH}_{2} \mathrm{O}$ treatment was responsible for the slower dissociation kinetics. However, previous studies have observed accelerated kinetics of ligand exchange between hydroxamate siderophores at lower $\mathrm{pH}$, suggesting a role for hydrogen ions in facilitating the release of ligands from the inner coordination sphere of iron [Raymond et al., 1984]. Instead, it is possible that the high ionic strength of seawater $(0.7 \mathrm{M})$ compared to $\mathrm{qH}_{2} \mathrm{O}$ induces changes in the activity of iron and siderophores, resulting in faster kinetics. As one potential mechanism, cations in seawater may facilitate iron exchange much like $\mathrm{H}^{+}$by releasing a ligand from the inner coordination sphere of the metal. While the dissociation of iron from hydroxamate siderophores is accelerated at high ionic strength, the association rate may be reduced. The exchange of iron from citrate to ferrioxamine $\mathrm{B}$ decreases at higher concentrations of $\mathrm{NaCl}$ [Ito et al., 2015]. Ito et al. 2015 demonstrated that $\mathrm{NaCl}$ inhibits the adjunctive pathway by slowing the direct association of 
ferrioxamine B with the mono-citrate complex. Thus, salinity has a dual effect on decreasing the efficacy of hydroxamate siderophores as iron binding chelates.

These results demonstrate that iron bound by ferrioxamine B and ferrichrome is not readily exchanged in dilute seawater, and that the turnover times of iron within these chelates exceed 135 days. In nature, photochemical or biological reductive mechanisms or other strong ligands may be responsible for enhancing the dissociation of iron from hydroxamate siderophores [Croot and Heller, 2012; Miethke, 2013].

The results for model compounds demonstrate that ${ }^{57} \mathrm{Fe}$ exchange monitored by mass spectrometry is a robust approach for determining metal dissociation kinetics. One of the primary advantages of this method is that it provides a compound specific dissociation rate of each component in a mixture such as natural seawater, whereas competitive ligand exchange provides a single measurement that reflects a combination of dissociation kinetics for all ligands. Thus, the mass spectrometry approach can be used to measure interactions and relative kinetics of multiple chelators within a single experiment. These kinetic measurements are related to ligand binding strength, which could potentially be used to determine the strength of unknown ligands through the principal of microscopic reversibility if the association rate can be measured or estimated. Another major benefit is that iron exchange can be monitored in very dilute solutions including natural seawater. Less than 1 picomole of compound is needed for each measurement. Extraction volumes can be scaled to liter quantities for natural seawater samples containing picomolar concentrations of organic ligand. This will enable the determination of environmentally relevant rates of iron dissociation that can be used in kinetic biogeochemical models of iron cycling.

The major current drawback of this approach is the long analysis time for each measurement (extraction/preconcentration time plus 30-60 min per time point). As a result, it is only applicable to monitoring species with slow exchange rates $(>1 \mathrm{hr})$, such as siderophores. Future methodological improvements could use ultrahigh pressure chromatography to achieve the same separation within minutes. Another potential source of error in this method is that the preconcentrated ligands are capable of exchanging iron with each other, which would drive the 
isotopic composition of each compound (and thus the calculated dissociation rates) closer together. One advantage of analyzing strong hydroxamate siderophores is that the adjunctive mechanisms are slow relative to the timescale of analysis. Earlier studies found that the exchange of iron between concentrated $(4 \mathrm{mM})$ siderophores ferrioxamine B and ferrichrome is only $50 \%$ complete after 220 hours of incubation at $25^{\circ} \mathrm{C}$ and $\mathrm{pH} 7.4$ [Raymond et al., 1984]. For analyzing more labile species, this source of error can be minimized by analyzing samples soon after extraction.

\subsubsection{Fe exchange of natural organic ligands:}

As a first application of this method, I investigated the iron exchange rates of organic extracts from the California Current System, described in the previous chapter. In the surface waters of the upwelling transition zone, there were samples that contained a mixture of unresolved dissolved organic matter (DOM), known siderophores (amphibactins), and an unknown iron binding ligand with $\mathrm{m} / \mathrm{z}$ 709. A ${ }^{57} \mathrm{Fe}$ exchange experiments were performed in order to gain insight into the relative binding strengths of these compounds and the timescales required for incorporation of an isotopic label. A large excess of ${ }^{57} \mathrm{Fe}$ citrate was added to the concentrated organic extract and was allowed to exchange with the natural seawater ligands for 30 minutes, 4 days, and 10 days before analysis by LC-ICPMS. ${ }^{56} \mathrm{Fe}^{\mathrm{Fe}} \mathrm{Ftal}_{\text {tot }}$ ratios were determined for the unresolved DOM eluting between 6 to 22 minutes, the amphibactins eluting between 25-28 minutes, and the unknown siderophore with $\mathrm{m} / \mathrm{z} 709$ eluting between 28.2-29 minutes (Fig. 6.4).

The chromatographically unresolved organic matter had a significantly slower dissociation rate than the siderophores. By the first timepoint (30 minutes), over half of this pool had exchanged ${ }^{56} \mathrm{Fe}$ for ${ }^{57} \mathrm{Fe}$ (Fig. 6.4). The siderophores took longer, with the $709 \mathrm{~m} / \mathrm{z}$ compound exchanging within 4 days and the amphibactins exchanging within 12 days. Since the dissociation kinetics for each compound are inversely related to the stability of the iron bound form, this result suggests that the unresolved compounds have a weaker affinity for iron compared with the amphibactins and the $709 \mathrm{~m} / \mathrm{z}$ siderophore. Furthermore, the amphibactins are stronger siderophores than the $709 \mathrm{~m} / \mathrm{z}$ compound, which may determine their relative effectiveness of acquiring iron under conditions of scarcity. 
The results from this study reflect iron exchange rates within the organic extract. The low salt concentration in the extract likely slows the iron dissociation rate relative to seawater conditions, as described above for the model siderophores. However, the preconcentrated organic extract also contained significantly higher concentrations of natural iron ligands (10,000x seawater) plus $0.2 \mathrm{mM}$ of citrate. These high concentrations can potentially greatly accelerate the rates of iron exchange by adjunctive mechanisms. As a result, the rates of iron exchange for these compounds may be much slower under natural conditions compared to the rates observed here. Despite this caveat, the rates observed in this study may be considered a lower bound to dissociation rates in the environment and suggest slow dissociation (on the order of days) for naturally occurring siderophores. This is consistent with our results for well-defined siderophores (above) and previous reports on the dissociation kinetics of siderophores. In contrast, unresolved DOM exchanges much faster (minutes to hours). These weaker compounds may be the same weak ligands (e.g. humic and fulvic acids, acidic polysaccharides) that are commonly measured in seawater by electrochemical methods [Gledhill and Buck, 2012]. In both our studies using LCICPMS and the electrochemical studies using competitive ligand exchange, the weaker ligands tend to be the most abundant fraction of the ligand pool. Future experiments are needed to directly link the structural compound classes detected by LC-ICPMS (unresolved DOM, siderophores) to compound classes determined by binding strength determined by electrochemical competitive ligand exchange measurements $\left(\mathrm{L}_{1}, \mathrm{~L}_{2}\right)$. This can be achieved by isolating fractions of unresolved DOM and unknown siderophores by liquid chromatography and determine the binding strength of each component in seawater using electrochemical measurements.

\subsection{Conclusions:}

The mechanisms that underlie the kinetics of metal exchange in seawater are extraordinarily complex due to the diverse nature of iron binding ligands and cations. Simplified laboratory experiments have been instrumental to the development of mechanistic models, of metal 
exchange in natural waters [Hering and Morel, 1989, 1990]. To validate these models, methods are needed to observe these rates under close-to-natural conditions.

Prior to this work, electrochemical experiments have been one of the only means of experimentally examining the chemical properties of natural metal complexes in marine environments. Here, we have described a novel mass spectrometry based approach that validates and expands upon these prior studies of the properties of natural ocean ligands. The use of isotopic exchange monitored by LC-ICPMS is unique in that exchange rates for multiple ligands can be determined independently within a single analysis, making it possible to track individual components of a complex environmental sample.

The results from this study demonstrate that the hydroxamate siderophores ferrioxamine $\mathrm{E}$ and ferrichrome have very slow dissociation kinetics in seawater in the absence of biological and photochemical mechanisms, suggesting that once bound to siderophores, microbes can only access this iron by active uptake through dedicated membrane receptors or by iron reduction. Further work is needed to carefully investigate the role of second order disjunctive mechanisms and the potential role of weaker binding molecules as iron shuttles to facilitate removal of iron from strong complexes. These processes may be important even in dilute seawater, and can be studied by varying the concentration of iron, citrate, and natural ligands added to a sample during incubation.

This work also demonstrates that long equilibration times are needed to label the strongest naturally occurring ligands with rare or radioactive isotopes of iron for incubation and uptake experiments if the ligands from seawater are fully saturated with iron. For weak iron binding compounds such as unresolved DOM, several hours is sufficient. Stronger complexes such as amphibactins require longer time periods of days to weeks. Finally, this method is useful for investigating the relative metal binding strengths of compounds isolated from seawater - an important characteristic that is unobtainable by other methods. Since many isotopes of many different metals can be detected in a single run, this method also opens opportunity to investigate rates of metal substitution and competition (e.g. copper and iron) for each component of natural organic ligands. 


\subsection{References:}

De Baar, H., P. Boyd, K. Coale, and M. Landry (2005), Synthesis of iron fertilization experiments: From the Iron Age in the Age of Enlightenment, J. Geophys. Res, 110(C9), 124, doi:10.1029/2004JC002601.

Baker, A. R., and P. L. Croot (2010), Atmospheric and marine controls on aerosol iron solubility in seawater, Mar. Chem., 120(1-4), 4-13, doi:10.1016/j.marchem.2008.09.003.

Boyd, P. W. et al. (2007), Mesoscale Iron Enrichment Experiments 1993-2005: Synthesis and Future Directions, Science, 315(February), 612-617.

Boyd, P. W., E. Ibisanmi, S. G. Sander, K. a. Hunter, and G. a. Jackson (2010), Remineralization of upper ocean particles: Implications for iron biogeochemistry, Limnol. Oceanogr., 55(3), 1271-1288, doi:10.4319/1o.2010.55.3.1271.

Boyd, P. W., R. F. Strzepek, M. J. Ellwood, D. A. Hutchins, S. D. Nodder, B. S. Twining, and S. W. Wilhelm (2015), Why are biotic iron pools uniform across high- and low-iron pelagic ecosystems?, Global Biogeochem. Cycles, 29(7), 1028-1043, doi:10.1002/2014GB005014.

Bundy, R. M., D. V Biller, K. N. Buck, K. W. Bruland, and K. A. Barbeau (2014), Distinct pools of dissolved iron-binding ligands in the surface and benthic boundary layer of the California Current, Limnol. Oceanogr., 59(3), 769-787, doi:10.4319/1o.2014.59.3.0769.

Croot, P. L., and M. I. Heller (2012), The importance of kinetics and redox in the biogeochemical cycling of iron in the surface ocean, Front. Microbiol., 3(June), 1-15, doi:10.3389/fmicb.2012.00219.

Gerringa, L. J. A., M. J. A. Rijkenberg, H. T. Wolterbeek, T. G. Verburg, M. Boye, and H. J. W. de Baar (2007), Kinetic study reveals weak Fe-binding ligand, which affects the solubility of $\mathrm{Fe}$ in the Scheldt estuary, Mar. Chem., 103(1-2), 30-45, doi:10.1016/j.marchem.2006.06.002.

Gledhill, M., and K. N. Buck (2012), The organic complexation of iron in the marine environment: a review., Front. Microbiol., 3, 1-17, doi:10.3389/fmicb.2012.00069.

Gledhill, M., P. McCormack, S. Ussher, E. P. Achterberg, R. F. C. Mantoura, and P. J. Worsfold (2004), Production of siderophore type chelates by mixed bacterioplankton populations in nutrient enriched seawater incubations, Mar. Chem., 88(1-2), 75-83, doi:10.1016/j.marchem.2004.03.003.

Hayes, C. T., J. N. Fitzsimmons, E. A. Boyle, D. McGee, R. F. Anderson, R. Weisend, and P. L. Morton (2015), Thorium isotopes tracing the iron cycle at the Hawaii Ocean Time-series Station ALOHA, Geochim. Cosmochim. Acta, 169, 1-16, doi:10.1016/j.gca.2015.07.019. 
Hering, J., and F. Morel (1990), Kinetics of trace metal complexation: ligand-exchange reactions, Environ. Sci. Technol., 252(9), 242-252, doi:0013-936X/90/0924-0242.

Hering, J. G., and F. M. M. Morel (1989), Slow coordination reactions in seawater, Geochim. Cosmochim. Acta, 53(3), 611-618, doi:10.1016/0016-7037(89)90004-5.

Ito, H., M. Fujii, Y. Masago, T. D. Waite, and T. Omura (2015), Effect of ionic strength on ligand exchange kinetics between a mononuclear ferric citrate complex and siderophore desferrioxamine B, Geochim. Cosmochim. Acta, doi:10.1016/j.gca.2015.01.020.

Johnson, K., K. Coale, V. Elrod, and N. Tindale (1994), Iron photochemistry in seawater from the equatorial Pacific, Mar. Chem., 46, 319-334.

Kieber, R. J., S. a Skrabal, B. J. Smith, and J. D. Willey (2005), Organic complexation of Fe(II) and its impact on the redox cycling of iron in rain., Environ. Sci. Technol., 39(6), 1576-83.

Kuma, K., J. Nishioka, and K. Matsunaga (1996), Controls on iron(III) hydroxide solubility in seawater: The influence of $\mathrm{pH}$ and natural organic chelators, Limnol. Oceanogr., 41(3), 396-407, doi:10.4319/1o.1996.41.3.0396.

Kuma, K., A. Katsumoto, H. Kawakami, F. Takatori, and K. Matsunaga (1998), Spatial variability of $\mathrm{Fe}(\mathrm{III})$ hydroxide solubility in the water column of the northern North Pacific Ocean, Deep - Sea Res. Part I - Oceanogr. Res. Pap., 45(1), 91-113, doi:10.1016/S09670637(97)00067-8.

Laglera, L. M., and M. Filella (2014), The relevance of ligand exchange kinetics in the measurement of iron speciation by CLE-AdCSV in seawater, Mar. Chem., doi:10.1016/j.marchem.2014.09.005.

Liu, X., and F. Millero (2002), The solubility of iron in seawater, Mar. Chem., 77, 43-54.

Macrellis, H. M., C. G. Trick, E. L. Rue, G. Smith, and K. W. Bruland (2001), Collection and detection of natural iron-binding ligands from seawater, Mar. Chem., 76(3), 175-187, doi:10.1016/S0304-4203(01)00061-5.

Mahowald, N., and S. Engelstaedter (2009), Atmospheric Iron Deposition: Global Distribution, Variability, and Human Perturbations*, Ann. Rev. Mar. Sci., 1, 245-78, doi:10.1146/annurev.marine.010908.163727.

Martin, J. H., R. M. Gordon, and S. E. Fitzwater (1991), The case for iron, Limnol. Oceanogr., 36(8), 1793-1802.

Martin, J. H. et al. (1994), Testing the iron hypothesis in ecosystems of the equatorial Pacific Ocean, Nature, 371(6493), 123-129.

Mawji, E., M. Gledhill, J. A. Milton, G. A. Tarran, S. Ussher, A. Thompson, G. A. Wolff, P. J. 
Worsfold, and E. P. Achterberg (2008), Hydroxamate siderophores: occurrence and importance in the Atlantic Ocean., Environ. Sci. Technol., 42(23), 8675-80.

Miethke, M. (2013), Molecular strategies of microbial iron assimilation: from high-affinity complexes to cofactor assembly systems., Metallomics, 5(1), 15-28, doi:10.1039/c2mt20193c.

Raymond, K. N., G. Mueller, B. Matzanke, and G. Müller (1984), Complexation of iron by siderophores a review of their solution and structural chemistry and biological function, Struct. Chem., 123, 49-102, doi:10.1021/ba-1977-0162.ch002.

Rue, E., and K. Bruland (1995), Complexation of iron(III) by natural organic ligands in the Central North Pacific as determined by a new competitive ligand equilibration/adsorptive cathodic stripping voltammetric method, Mar. Chem., 50(1-4), 117-138, doi:10.1016/03044203(95)00031-L.

Rue, E. L., and K. W. Bruland (1997), The role of organic complexation on ambient iron chemistry in the equatorial Pacific Ocean and the response of a mesoscale iron addition experiment, Limnol. Oceanogr., 42(5), 901-910, doi:10.4319/lo.1997.42.5.0901.

Velasquez, I., B. L. Nunn, E. Ibisanmi, D. R. Goodlett, K. A. Hunter, and S. G. Sander (2011), Detection of hydroxamate siderophores in coastal and Sub-Antarctic waters off the South Eastern Coast of New Zealand, Mar. Chem., 126(1-4), 97-107, doi:10.1016/j.marchem.2011.04.003.

Vraspir, J. M., and A. Butler (2009), Chemistry of Marine Ligands and Siderophores, Ann. Rev. Mar. Sci., 1(1), 43-63, doi:10.1146/annurev.marine.010908.163712.

Witter, A. E., and G. W. Luther (1998), Variation in Fe-organic complexation with depth in the Northwestern Atlantic Ocean as determined using a kinetic approach, Mar. Chem., 62(3-4), 241-258, doi:10.1016/S0304-4203(98)00044-9.

Witter, A. E., D. A. Hutchins, A. Butler, and G. W. L. Iii (2000), Determination of conditional stability constants and kinetic constants for strong model Fe-binding ligands in seawater, Mar. Chem., 1-17.

$\mathrm{Wu}$, J., and G. Luther (1995), Complexation of Fe(III) by natural organic ligands in the Northwest Atlantic Ocean by a competitive ligand equilibration method and a kinetic approach, Mar. Chem., 50(1-4), 159-177, doi:10.1016/0304-4203(95)00033-N. 


\subsection{Figures:}
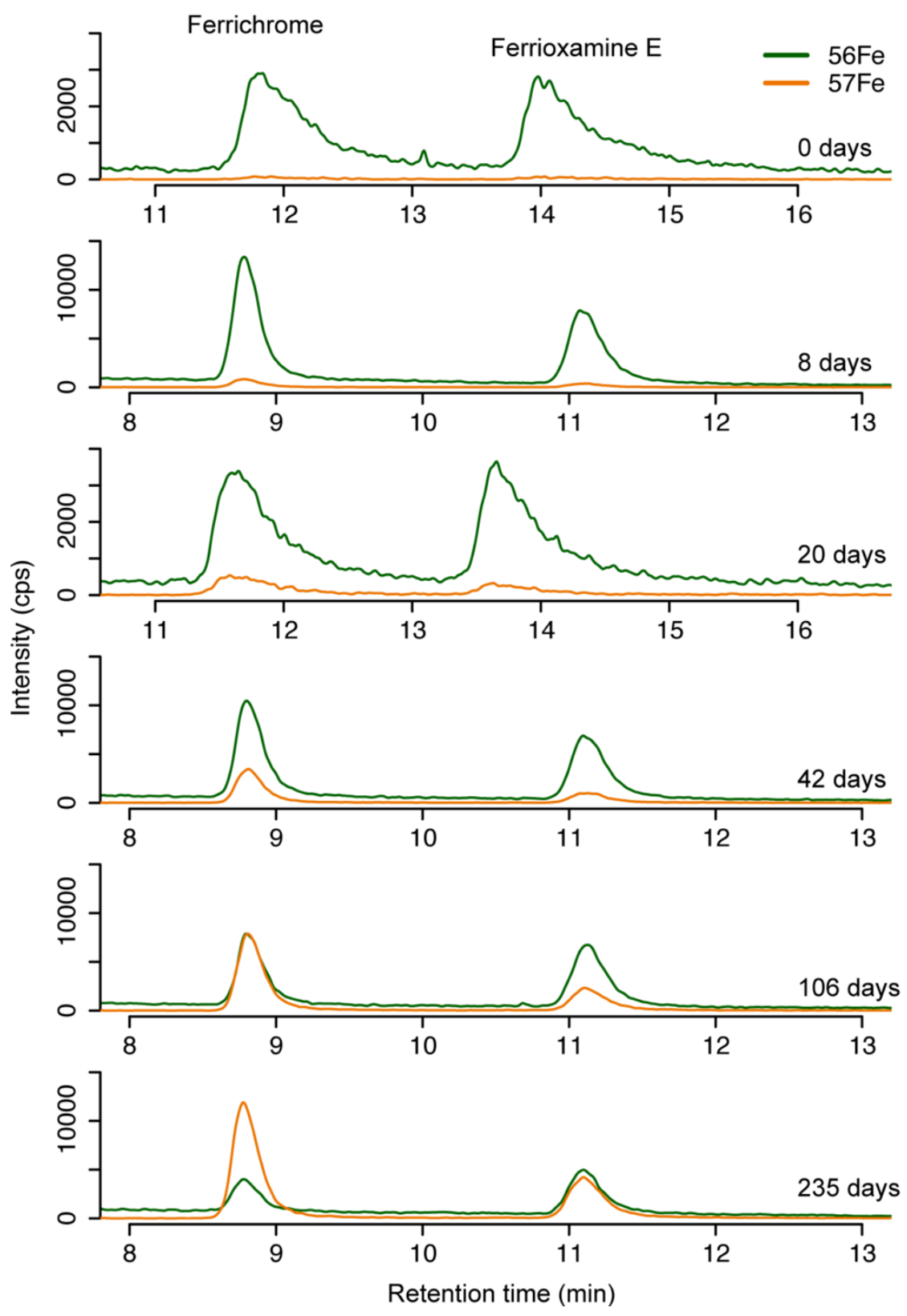

Figure 6.1: Seawater exchange: LC-ICPMS separation and detection of ferrichrome and ferrioxamine E extracted from seawater incubations with excess ${ }^{57} \mathrm{Fe}$ added. Note that the samples from 0 and 20 days were analyzed at a different time than the other four samples using a different LC column, hence the differences in peak shape, sensitivity, and retention time compared to the other four samples. Over time, ${ }^{56} \mathrm{Fe}$ is lost from the compounds and replaced with ${ }^{57} \mathrm{Fe}$. 

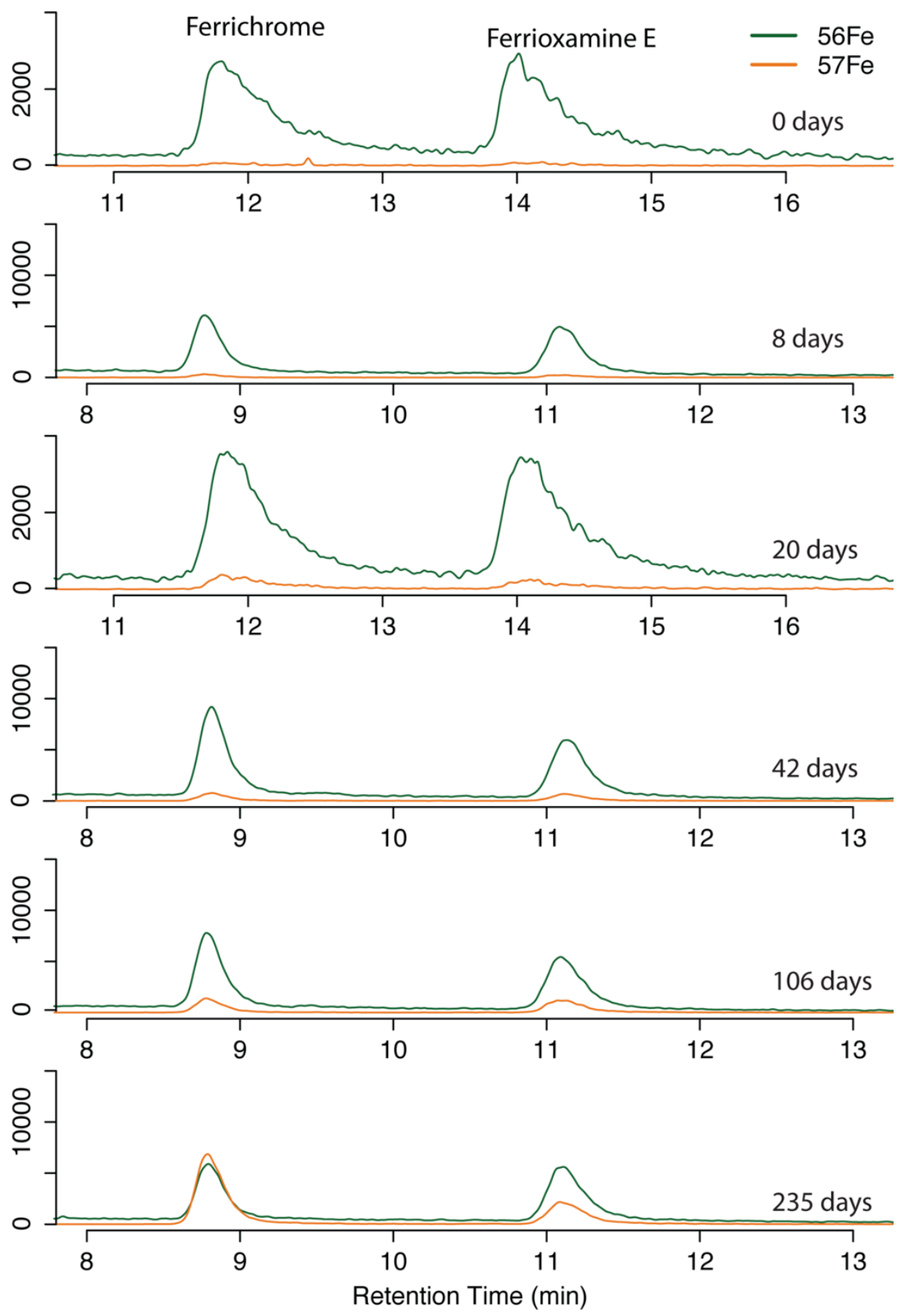

Figure 6.2: $\mathrm{H}_{2} \mathrm{O}$ exchange: LC-ICPMS separation and detection of ferrichrome and ferrioxamine $\mathrm{E}$ extracted from water incubations with excess ${ }^{57} \mathrm{Fe}$ added. The dissociation kinetics were slower in water compared with seawater. 

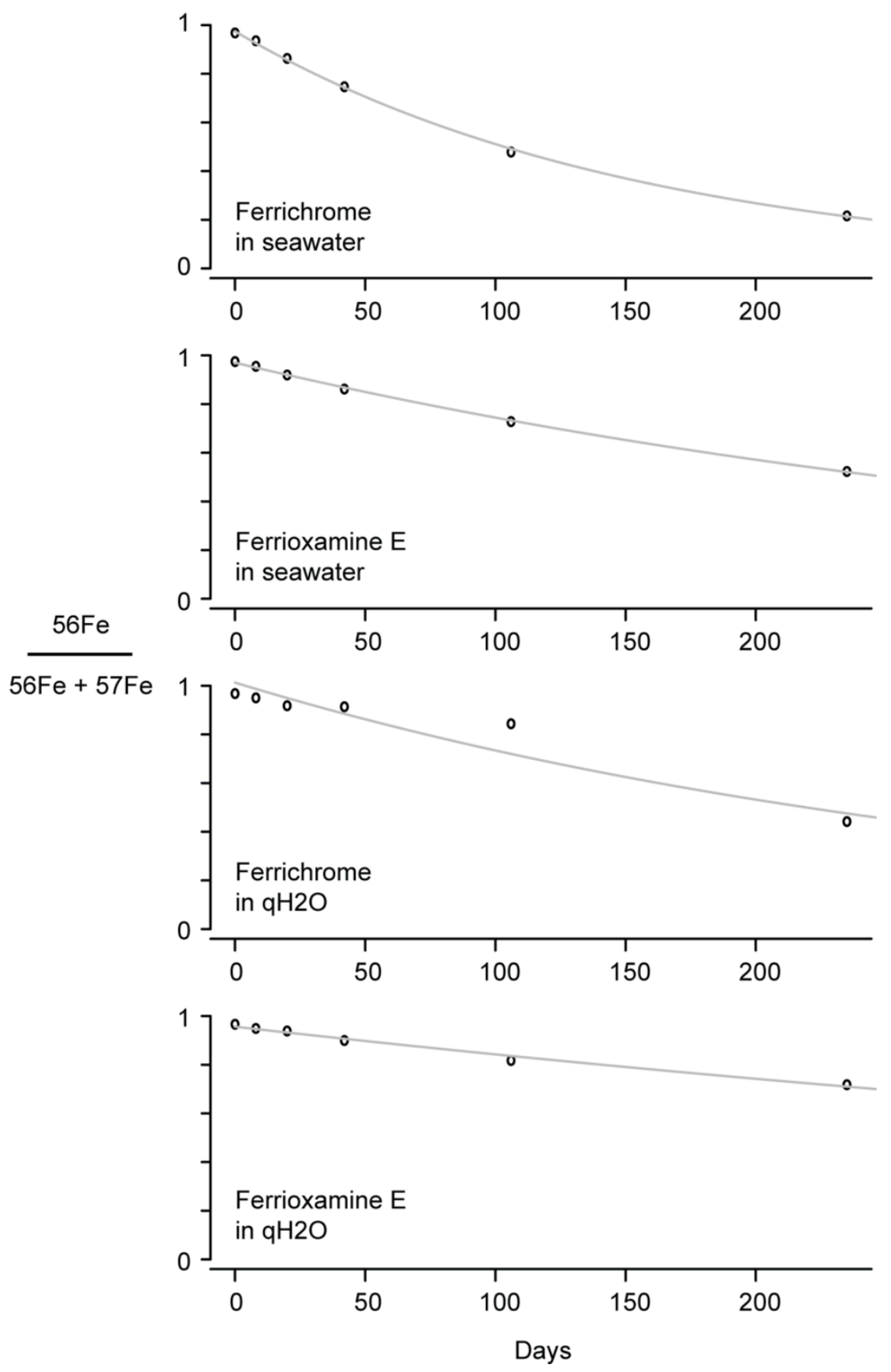

Figure 6.3: Isotopic ratios of ferrichrome and ferrioxamine E over time measured by LC-ICPMS during an incubation with ${ }^{57} \mathrm{Fe}$ citrate in either seawater or $\mathrm{qH}_{2} \mathrm{O}$. An exponential decay curve (gray line) in the form of equation 9 was fit to the data to determine the dissociation constants of each compound under each condition. 
a
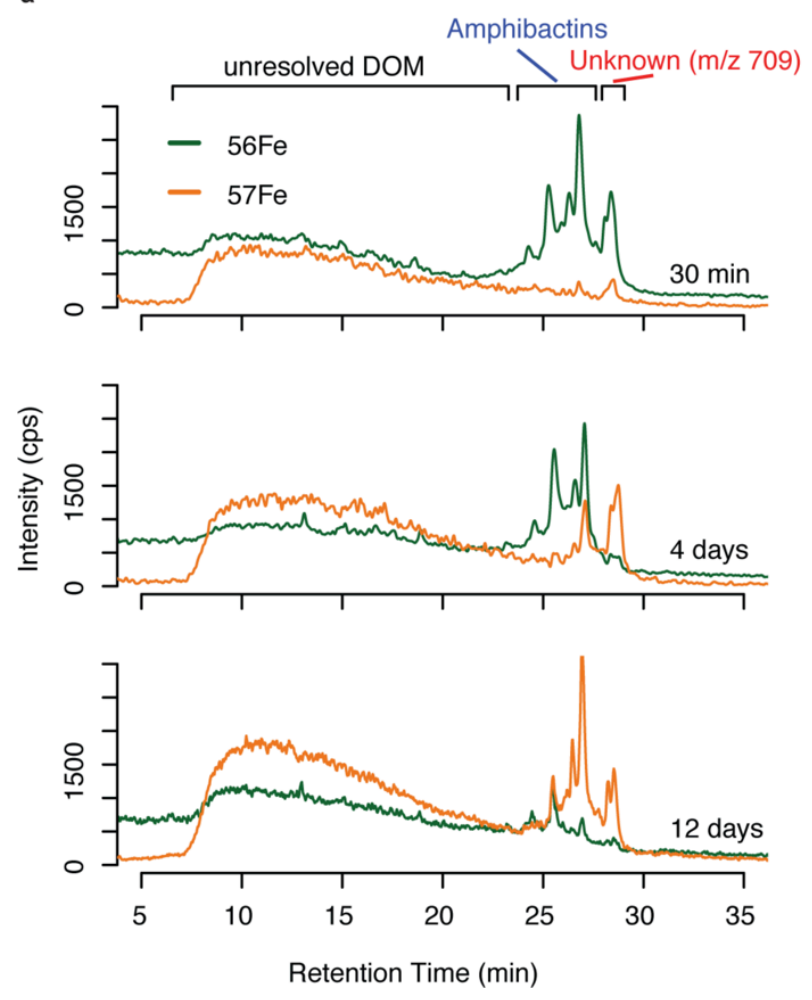

b

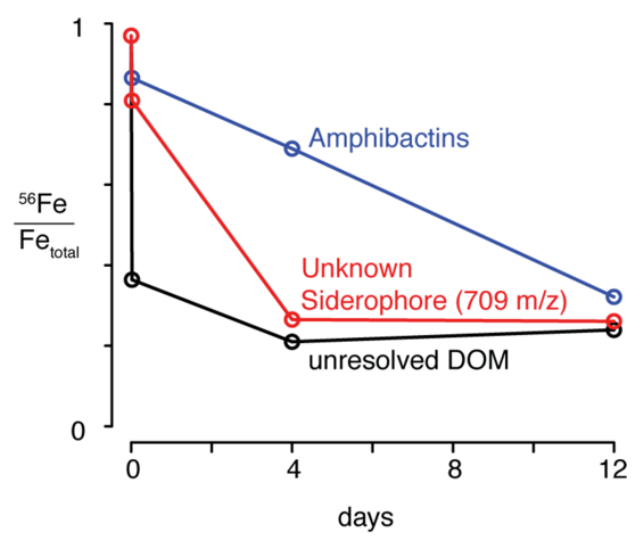

Figure 6.4: (a) Separation of natural iron ligands by LC-ICPMS over the course of isotopic exchange with ${ }^{57} \mathrm{Fe}$-citrate. Over time, ${ }^{56} \mathrm{Fe}$ dissociates from the natural ligands and is replaced with ${ }^{57} \mathrm{Fe}$. (b) ${ }^{56} \mathrm{Fe}$ fraction associated with each component of the natural ligand sample over time. The relative dissociation rates for these compounds suggest that the iron binding strength with iron is greatest for amphibactin, followed by the unknown siderophore $(709 \mathrm{~m} / \mathrm{z})$, and that the unresolved DOM has a comparatively weaker iron affinity. 
Table 6.1: Siderophore dissociation rates

\begin{tabular}{lllll}
\hline Conditions & Siderophore & $\begin{array}{l}\mathrm{k}_{\mathrm{d}}(\mathrm{s}-1) \\
\times 10^{-8}\end{array}$ & $\begin{array}{l}\text { std error }\left(\mathrm{s}^{-1}\right) \\
\times 10^{-8}\end{array}$ & $\begin{array}{l}\mathrm{published}^{\mathrm{a}} \mathrm{k}_{\mathrm{d}} \\
\left(\mathrm{s}^{-1}\right) \times 10^{-8}\end{array}$ \\
$\begin{array}{l}\text { Seawater } \\
\mathrm{pH} \mathrm{8}\end{array}$ & $\begin{array}{l} \\
\text { Ferrichrome }\end{array}$ & 8.4 & 0.095 & $5 \pm 4$ \\
& $\begin{array}{l}\text { Ferrioxamine E } \\
\text { Ferrioxamine B }\end{array}$ & 3.4 & 0.037 & $150 \pm 180$ \\
& & & \\
$\mathrm{qH}_{2} \mathrm{O}$ & & & \\
$\mathrm{pH} \mathrm{5}$ & Ferrichrome & 4.2 & 0.55 & \\
& Ferrioxamine E & 1.6 & 0.084 &
\end{tabular}

${ }^{\mathrm{a}}$ Witter et. al. 2000 


\section{Chapter 7: Conclusions and Future Directions}

\subsection{Summary}

The organic speciation of trace metals in the ocean has remained one of the major uncertainties in our understanding of metal fluxes in the ocean and the links between metal and carbon cycling. To address this challenge, there has been a significant need for methods that are capable of determining the molecular level composition of metal binding ligands directly from environmental samples. While numerous studies provide indirect evidence that strong iron ligands/siderophores exist and impact biological processes in iron deficient regions of the ocean characterized by high nitrate and phosphate, there has yet to be a study that provides direct characterization of the identity of these compounds. Progress has historically been limited by the insufficient sensitivity of characterization techniques when applied to the oligotrophic surface ocean.

The methodological arsenal presented in this thesis has overcome this challenge and provided new insight into the chemical processes that link microbial communities to marine iron cycling. New trace metal clean chromatography methods coupled with inductively coupled plasma mass spectrometry (ICPMS) enable the quantification of $10^{-13}$ mole of iron binding ligand by eliminating high methodological contamination. This has made it possible for the first time to detect and quantify bound versus unbound concentrations of specific iron binding siderophores present in natural seawater. This method generates molecular targets of interest and guides their structural characterization by high resolution electrospray ionization mass spectrometry (ESIMS) techniques. The isotope pattern screening algorithms introduced in chapter 3 provides a rapid means for determining the ionic mass of the intact metal complexes, which can then be targeted for $\mathrm{MS}^{2}$ fragmentation to obtain a diagnostic fingerprint of these compounds that can be compared to compound libraries. In many cases, the isotopologues of metal containing compounds are poorly resolved in ESIMS scans due to their low abundance and ion suppression effects of complex samples. At the same time, complex samples often have coeluting features that happen to mimic metal isotope patterns (false positives). These effects make it very challenging to confidently assign masses to each feature detected by LC-ICPMS, particularly when the compounds are unknown and cannot be verified by $\mathrm{MS}^{2}$ fragmentation patterns. The 
rare-isotope exchange methods introduced in chapters 4 and 6 provide an essential means for validating these masses, enabling confident characterization of novel compounds directly from seawater. Monitoring the kinetics of isotope exchange by LC-ICPMS has also proven to be a useful approach for comparing the relative metal binding strengths of natural siderophores in seawater, even when their absolute identity is unknown.

Applying these methods to sites across the ocean has demonstrated for the first time the diversity of siderophores across major biomes of the Pacific Ocean. Of note, amphibactins were identified as a common feature of iron starved high nitrate waters associated with coastal upwelling regions in the Pacific Ocean. Based on these results, it seems likely that siderophore mediated iron acquisition pathways are particularly important in iron-deficient regions of the ocean where dissolved iron concentrations are low $(<0.2 \mathrm{nM})$ and dissolved nitrate is high $(>5 \mu \mathrm{M})$. We propose that microbial communities adapt their iron acquisition strategies in order to remain competitive in these iron-scarce regions. Producing amphiphilic siderophores may avoid the diffusive loss that typifies classic siderophores such as ferrioxamines. In other environments, such as within nutrient rich sinking particles, the stronger iron binding strength of ferrioxamines relative to amphiphilic siderophores (unpublished data) may favor ferrioxamine production.

Other siderophores, the synechobactins, appear to be associated with benthic environments. Finally, masses and fragmentation patterns were determined for numerous compounds that do not appear in compound databases. These include iron, nickel, copper, and bromine containing compounds found across the surface ocean and from zooplankton grazing incubations. Although the complete structural identity of these compounds is currently unknown, we have enough information (namely $\mathrm{MS}^{2}$ fragmentation patterns) to include them in mass spectral libraries. Thus, it will be possible to identify the same compounds in other regions of the ocean or in cultures (as was the case for the unknown siderophore with $\mathrm{m} / \mathrm{z} 709$, found during both the GEOTRACES and IRNBRU cruises). As these libraries continue to grow, it is likely that the parent organisms and biosynthetic pathways for these unknown compounds will be discovered, enabling us to link them to specific biological processes. Moving forward, the compilation of these libraries will be important as more measurements are made across multiple laboratories. 


\subsection{Next steps}

One of the immediate next steps will be to study the impact of siderophores identified from the marine environment on iron bioavailability. One approach is to identify the genes involved in the uptake of these siderophores in the marine environment and look for them in the environment, as was done in Chapter 4 for amphibactin biosynthesis. Proteomic approaches may be able to identify the transporters involved in amphibactin uptake by determining which genes are co-regulated with amphibactin biosynthesis as the bacteria is grown under different iron conditions. By confidently identifying genes involved in siderophore processing in the lab and then looking for homologous sequences in ocean metagenomic data, it will be possible to target environmentally relevant gene sequences. Single cell genomes may also allow us to connect these genes to specific organisms. Another approach is to perform tracer uptake experiments with siderophores bound to radioisotopes of iron. These compounds can be added to natural seawater and the cells that take the compounds up can be sorted by flow cytometry and detected by scintillation counting or they can be labeled with fluorescent tags and detected by autoradiography.

Another major challenge will be to expand the analytical window of LCMS based methods by altering the extraction procedures used to isolate the organic matter. Acidification of samples prior to extraction is likely to isolate a larger fraction of dissolved organic compounds, potentially including polar siderophores. In addition, elution methods with more nonpolar solvents or solvents at acidic or basic $\mathrm{pH}$ may release more iron/ligand from the extraction column than a simple methanol or acetonitrile elution. In both cases, new chromatographic conditions may be required to optimize the separation of these fractions. This work should begin with developing procedures for extracting model compounds of interest from seawater (e.g. enterobactin) and detecting the iron-bound form by LC-ICPMS, and then applying these new methods to seawater samples.

Further work is also required to identify the functional groups associated with the chromatographically unresolved organic matter that seems to account for the majority of the organic iron binding capacity in seawater. This is linked to the greater overarching challenge of 
characterizing marine DOM more generally. One potentially promising approach for determining the molecular formula of DOM molecules that bind metals is the use of ultrahigh resolution fourier transform ion cyclotron resonance mass spectrometry with $>1 \mathrm{e} 6$ mass resolution. These instruments are capable of resolving the fine isotopic structure of molecules containing $\mathrm{Fe}, \mathrm{C}, \mathrm{O}$ and N. Detecting the minor isotopes of these elements (e.g. ${ }^{15} \mathrm{~N},{ }^{54} \mathrm{Fe},{ }^{57} \mathrm{Fe}$ ) will be necessary to confidently infer their presence in molecules within such a complex mixture. Coupling this method with isotopic exchange of ${ }^{54} \mathrm{Fe}$ or ${ }^{57} \mathrm{Fe}$ for the naturally occurring Fe may be a valuable approach for increasing confidence that the Fe containing masses identified by naturally occurring isotopic patterns are genuine.

Another future goal will be to reduce the scale of chromatography used for LC-ICPMS-ESIMS. Currently, only $25 \%$ of the injected sample is directed to the LC-ICPMS, since flow rates above $50 \mu \mathrm{L} / \mathrm{min}$ of organic solvent increases the risk of extinguishing the plasma. Lower (microliter) flow rates through long capillary columns coupled with a total consumption nebulizer will increase the amount of sample that makes it into the plasma, boosting sensitivity and reducing sample requirements. Although quantitation may be negatively impacted by these low flow methods due to decreased nebulization stability prior to ICPMS analysis, longer columns may improve chromatographic separation, making it easier to identify each compound. Furthermore, the increased sensitivity will substantially decrease sample volume requirements. This has been a major limitation of the existing method, which involves the preconcentration of large volumes of trace metal clean seawater. Reducing the required volume to several liters will open new opportunities to sample high-resolution depth profiles using standard trace metal clean rosettes. This will enable detailed spatial mapping of siderophores and other iron binding metabolites across oceanographic sections and provide better constraints on the sources and sinks of these metabolites. 
